Review

\title{
Lipid Vesicles and Other Polymolecular Aggregates-From Basic Studies of Polar Lipids to Innovative Applications
}

\author{
Peter Walde $^{1, *(\mathbb{D})}$ and Sosaku Ichikawa ${ }^{2, *}$ \\ 1 Laboratory for Multifunctional Materials, Department of Materials, ETH Zürich, Vladimir-Prelog-Weg 5, \\ CH-8093 Zürich, Switzerland \\ 2 Faculty of Life and Environmental Sciences, University of Tsukuba, 1-1-1 Tennodai, Tsukuba 305-8572, \\ Ibaraki, Japan \\ * Correspondence: peter.walde@mat.ethz.ch (P.W.); ichikawa.sosaku.fn@u.tsukuba.ac (S.I.)
}

check for updates

Citation: Walde, P.; Ichikawa, S. Lipid Vesicles and Other Polymolecular Aggregates-From Basic Studies of Polar Lipids to Innovative Applications. Appl. Sci. 2021, 11, 10345. https://doi.org/ 10.3390/app112110345

Academic Editor: Hidehiko Hirakawa

Received: 29 September 2021

Accepted: 26 October 2021

Published: 3 November 2021

Publisher's Note: MDPI stays neutral with regard to jurisdictional claims in published maps and institutional affiliations.

Copyright: (c) 2021 by the authors. Licensee MDPI, Basel, Switzerland. This article is an open access article distributed under the terms and conditions of the Creative Commons Attribution (CC BY) license (https:// creativecommons.org/licenses/by/ $4.0 /)$.

\begin{abstract}
Lipid vesicles (liposomes) are a unique and fascinating type of polymolecular aggregates, obtained from bilayer-forming amphiphiles_or mixtures of amphiphiles-in an aqueous medium. Unilamellar vesicles consist of one single self-closed bilayer membrane, constituted by the amphiphiles and an internal volume which is trapped by this bilayer, whereby the vesicle often is spherical with a typical desired average diameter of either about $100 \mathrm{~nm}$ or tens of micrometers. Functionalization of the external vesicle surface, basically achievable at will, and the possibilities of entrapping hydrophilic molecules inside the vesicles or/and embedding hydrophobic compounds within the membrane, resulted in various applications in different fields. This review highlights a few of the basic studies on the phase behavior of polar lipids, on some of the concepts for the controlled formation of lipid vesicles as dispersed lamellar phase, on some of the properties of vesicles, and on the challenges of efficiently loading them with hydrophilic or hydrophobic compounds for use as delivery systems, as nutraceuticals, for bioassays, or as cell-like compartments. Many of the large number of basic studies have laid a solid ground for various applications of polymolecular aggregates of amphiphilic lipids, including, for example, cubosomes, bicelles or-recently most successfully-nucleic acids-containing lipid nanoparticles. All this highlights the continued importance of fundamental studies. The life-saving application of mRNA lipid nanoparticle COVID-19 vaccines is in part based on year-long fundamental studies on the formation and properties of lipid vesicles. It is a fascinating example, which illustrates the importance of considering (i) details of the chemical structure of the different molecules involved, as well as (ii) physical, (iii) engineering, (iv) biological, (v) pharmacological, and (vii) economic aspects. Moreover, the strong demand for interdisciplinary collaboration in the field of lipid vesicles and related aggregates is also an excellent and convincing example for teaching students in the field of complex molecular systems.
\end{abstract}

Keywords: liposome; lipid vesicle; lipid nanoparticle; phospholipid; phosphatidylcholine; phase diagram; micelle; bicelle; cubic phase; drug delivery

\section{Introduction}

The aggregation behavior of biological amphiphilic lipids in the presence of water as a function of water content and temperature has been the subject of numerous fundamental investigations over the last couple of decades. Binary and ternary phase diagrams were determined for many lipid/water systems to understand the situation at thermodynamic equilibrium. Furthermore, methods were elaborated for the reproducible preparation of metastable aqueous lipid dispersions, and their physico-chemical properties were investigated in great detail. Based on the many results that have been obtained from such studies, innovative applications emerged over the years, resulting not only in useful research tools, for example, as biomembrane model systems, but also in various lipid-based commercial products. One of the recent successes is the development of lipid nanoparticle (LNP)-based 
siRNA delivery systems [1,2] and COVID-19 vaccines containing as active ingredient a nucleoside-modified mRNA coding for the spike proteins of the COVID-19 virus ('CO' stands for corona, 'VI' for virus, and 'D' for disease) [3-5].

The development of $\sim 70-90 \mathrm{~nm}$-sized LNPs for the delivery of nucleic acids is the result of intensive research at universities and in industry, particularly focusing on the challenge to encapsulate-and thereby protect-nucleic acids in a particle that is non-toxic and efficiently taken up by target cells. Some of the essential steps of the work preceding commercialization were (i) the finding of a $\mathrm{pH}$-sensitive cationic amphiphile that is able to complex anionic nucleic acids without being toxic, (ii) the selection of amphiphiles that are able to stabilize the LNPs in vitro as well as in vivo after administration before the target cells are reached, and (iii) the assembly of the LNPs in a reproducible and reliable way to the desired size and morphology. While (i) and (ii) are chemical challenges that had to be dealt with, (iii) is the engineering part, which is just as important as the molecular composition. LNPs are a specific type of polymolecular aggregates which are related to similarly-sized lipid vesicles (liposomes). Historically, intensive fundamental research on the aggregation behavior of polar lipids carried out by many research groups worldwide laid a solid foundation for the development of LNPs containing internal nucleic acids. LNPs emerged from studies on lipid vesicles (liposomes), from many challenging attempts to incorporate DNA or RNA inside lipid vesicles, for example, [6-15]. There would probably be no LNPs without the broad basic knowledge accumulated over the years on the chemistry, biophysics, engineering (methods of controlled and reproducible preparation), pharmacology, and immunology of lipid vesicles. On the other hand, the field of lipid vesicles emerged from the many fundamental studies on the phase behavior of amphiphilic lipids in the presence of water.

In the first part of this review, we would like to recall some of the earlier pioneering investigations of the aggregation behavior of a few selected amphiphilic lipids in water (or an aqueous medium) to illustrate how the aggregation state primarily depends on the chemical structure of the amphiphile, on the amphiphile concentration, on the composition of the aqueous solution, and on temperature. Although all this might be trivial to most of the readers, we feel, however, that it is worth emphasizing the importance of careful and reliable fundamental research, without which the development of many commercial products probably would not have been possible. Some of the examples mentioned may also serve for teaching introductory courses on lipid aggregates, or they may motivate to search for other examples that significantly contributed to our current understanding of the aggregation behavior of the fascinating class of amphiphilic lipids and their increasing numbers of successful applications in different areas.

In the following, we will highlight a few of the many important fundamental studies on the behavior of amphiphilic lipids when they are brought in contact with water (or an aqueous solution). The focus will be on lipid solubility in water and lipid swelling behavior, self-assembly (i.e., aggregation) in water, aggregate structure and dynamics; see Chapter 2. These basic studies, often curiosity-driven [16,17], convincingly demonstrated that the lipid's aggregation state in an aqueous medium can be quite complex and does not depend only on the chemical structure and self-assembly propensity of the amphiphile but also on the amphiphile concentration, on the presence of other amphiphiles, or of hydrophobic or hydrophilic compounds, and on the temperature. Moreover, it was shown that details of the procedure with which a sample consisting of amphiphilic lipids and an aqueous medium is prepared, i.e., the method of preparation, often are very important for a desired application. Indeed, the majority of lipid aggregates that are of interest for applications are only kinetically stable and are not the thermodynamically most favored structures or a true equilibrium state [18]. They represent dispersed phases. Therefore, the way these polymolecular lipid aggregates are assembled is very important. Examples are phospholipid-based vesicles (Figure 1) [19,20], obtained by "guided" (i.e., "directed" or "engineered") assembly processes from bilayer-forming amphiphiles as colloidal lipid vesicle 
dispersions (see Chapter 4) which are useful, for example, as drug delivery systems [21-23]; see Chapter 6.

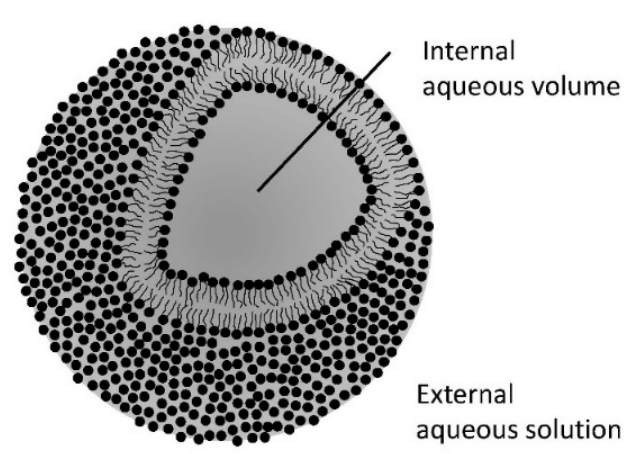

Lipid bilayer

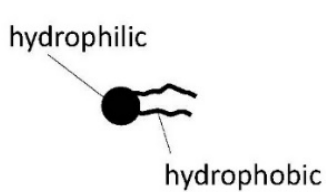

Amphiphilic lipid
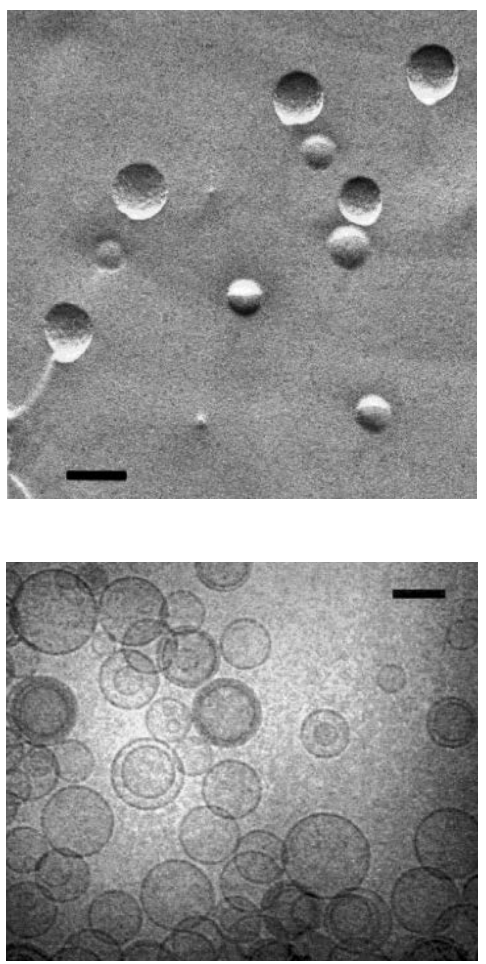

(b)

(a)

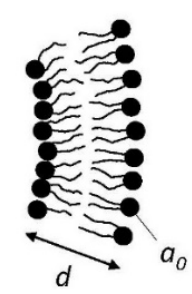

Figure 1. Phospholipid vesicles. (a) Schematic representation of a spherical unilamellar lipid vesicle. A self-closed bilayer of amphiphilic lipids forms the boundary of the vesicle, thereby entrapping a small internal aqueous volume. Assuming that the lipid is POPC, 1-palmitoyl-2-oleoyl-sn-glycero-3-phosphocholine, with an average head group area requirement $\left(a_{0}\right)$ of $0.63 \mathrm{~nm}^{2}$ and a bilayer thickness $(d)$ of $4 \mathrm{~nm}$ [19], a spherical unilamellar vesicle with a diameter of $100 \mathrm{~nm}$ consists of about $9.2 \times 10^{4}$ POPC molecules and has a trapped volume of about $4.08 \times 10^{-19} \mathrm{~L}$ per vesicle, corresponding to $2.7 \mu \mathrm{L} / \mu \mathrm{mol}$ POPC. For a dispersion of $1 \mathrm{mM}$ POPC as ideal unilamellar vesicles of $100 \mathrm{~nm}$ diameter, the calculated vesicle concentration is about $11 \mathrm{nM}$ and the total aqueous volume trapped by all vesicles present in $1 \mathrm{~mL}$ of this dispersion is $2.7 \mu \mathrm{L}$ only. (b) Freeze fracture electron microscopy (top) and cryo-TEM (bottom) images of a $20 \mathrm{mM}$ POPC dispersion $(50 \mathrm{mM} \mathrm{Tris/HCl}$, $p H=8.0$ ) prepared by polycarbonate membrane extrusion (final extrusions through membranes with $100 \mathrm{~nm}$ pores sizes, see Chapter 4) at $T=25^{\circ} \mathrm{C}$ (bar: $100 \mathrm{~nm}$ ). The vesicles were prepared in the presence of the highly water-soluble enzyme $\alpha$-chymotrypsin; after removal of free enzyme at the conditions used in this previous work, each vesicle contained on average 87 entrapped $\alpha$-chymotrypsin molecules (not visible on the electron microscopy images) [20]. The concentration of free, non-associated POPC was very low $\left(\sim 10^{-10} \mathrm{M}\right.$; see Section 2.15$)$. The electron microscopy images are reproduced with permission from [20], John Wiley \& Sons, 1999.

For any type of application of lipid vesicles and other dispersed polymolecular aggregates, e.g., cubosomes, the colloidal stability is one of the issues to consider; see Chapter 3. This stability depends on the chemical structures of the lipids used, on the composition as well as on the way the lipid aggregates are prepared. In addition to this latter engineering aspect concerning the preparation of dispersed lipid aggregates, further complexity and challenges arise if one aims to functionalize the vesicle surface, or if one aims to associate or entrap active molecules of interest within the aggregates for a desired application; see Chapter 5. Typical examples can be found in the field of lipid vesicle-based drug delivery systems [21-25] and cosmetic and dermatological products [26-32] or as vesicular cell model systems [33-39]; see Chapter 6.

Although the opinions may diverge, and serendipity often might play an important role, there are many examples where successful applications are based on an understand- 
ing that is based on fundamental studies, possibly carried out many years before the applications were demonstrated or commercialized. This has recently been emphasized by Dijkgraaf (2017) [40]: "Basic research, driven by curiosity, freedom and imagination, provides the groundwork for all applied research and technology".

The basic studies mentioned in this review are by no means the only ones in the highly interdisciplinary field of polymolecular lipid assemblies. The examples discussed are picked from the literature since we feel that they are among the many excellent, fundamental investigations that contributed significantly to our current level of understanding of lipid vesicles and other polymolecular aggregates and their applications in different areas.

\section{Comparison of Aqueous Dispersions of a Few Selected Biological Polar Lipids}

\subsection{Polar Lipid Classification}

On the basis of a large number of fundamental studies by different research groups on the behavior of lipids in the presence of water, notably of lipids carrying a polar head group at one end of the molecule (called "polar lipids"), an empirical lipid classification according to the physico-chemical properties of the lipids in bulk aqueous solution and at the water-air interface was proposed by Small [41]. This classification is illustrated here with the following biological polar lipids: Palmitic acid, oleic acid, sodium or potassium oleate, monoolein, diolein, oleoyl-lyso-PC, DOPC, egg PC, POPC, DPPC, DOPE, and DOPA (Figure 2 and Table 1) [42,43].
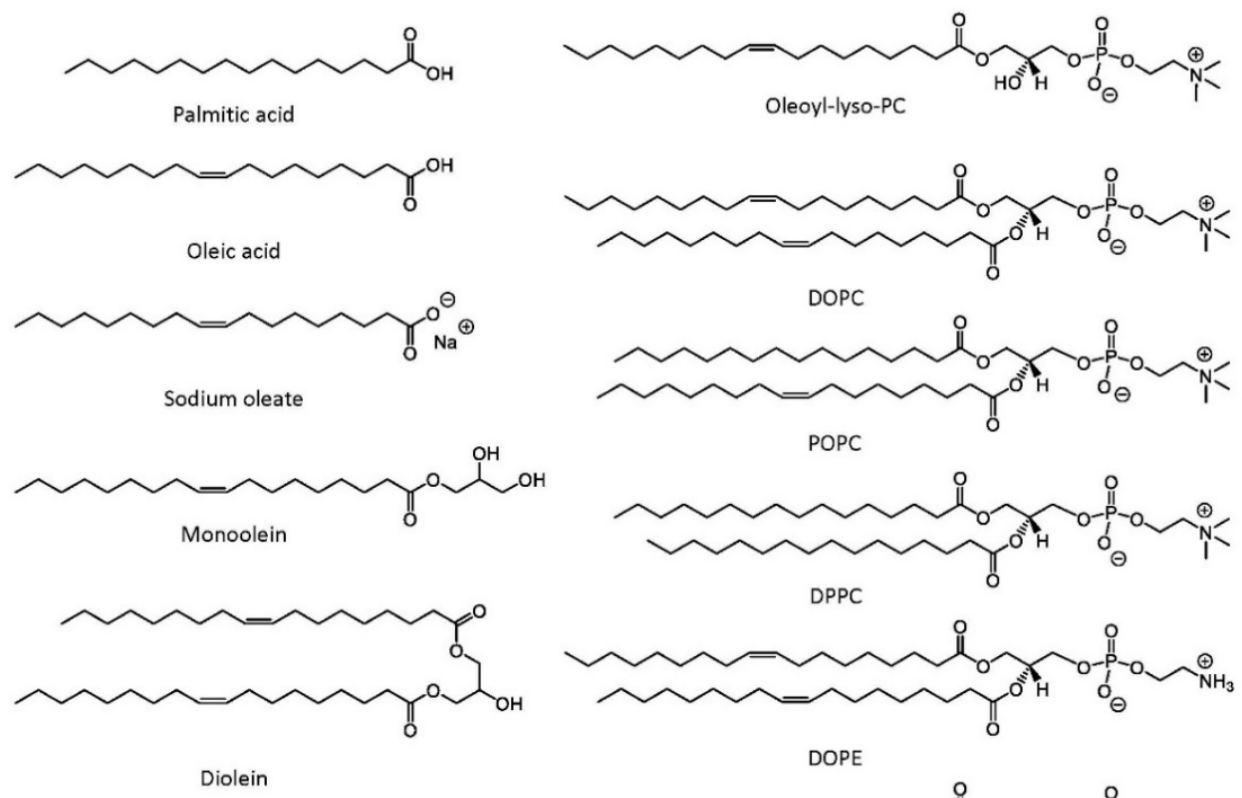

DOPC
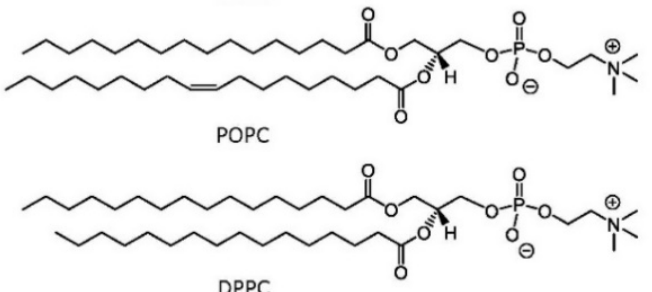

DPPC

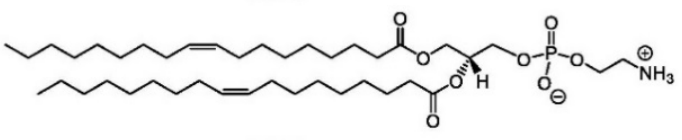

DOPE

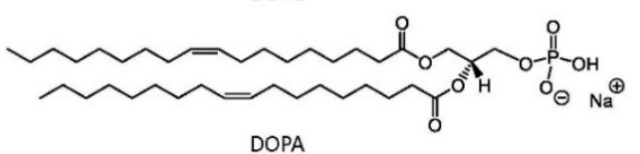

Figure 2. Chemical structures of some of the biological amphiphilic lipids mentioned in this review. Monoolein and diolein are considered to be racemic 1-monoolein and 1,3-diolein, respectively; DOPC, 1,2-dioleoyl-sn-glycero-3-phosphocholine; POPC, 1-palmitoyl-2-oleoyl-sn-glycero-3phosphocholine; DPPC, 1,2-dipalmitoyl-sn-glycero-3-phosphocholine; DOPE, 1,2-dioleoyl-sn-glycero3-phosphoethanolamine; DOPA, 1,2-dioleoyl-sn-glycero-3-phosphatidic acid sodium salt.

The compounds are chosen to illustrate how (i) a change in the polar head group and (ii) a variation of the hydrophobic chain(s)—saturated palmitic acid vs. unsaturated oleic acid-influence the concentration- and temperature-dependent aggregation behavior of the lipids in aqueous solution. Some of the key properties of the listed compounds when brought in contact with water are summarized in this section, illustrated with previously published binary or ternary phase diagrams and a number of electron microscopy images. 
Table 1. Examples of biological polar lipids, grouped according to the classification of Small [41].

\begin{tabular}{|c|c|c|}
\hline Polar Lipid $^{1}$ & Properties in Bulk Aqueous Solution & Properties at the Water-Air Interface \\
\hline $\begin{array}{l}\text { Class I } \\
\text { Palmitic acid } \\
\text { Oleic acid } \\
\text { Diolein }\end{array}$ & $\begin{array}{l}\text { Practically insoluble, } \\
\text { the molecules do not swell }\end{array}$ & Molecules spread to form stable monolayers \\
\hline $\begin{array}{c}\text { Class II } \\
\text { DOPC } \\
\text { POPC } \\
\text { egg PC } \\
\text { DPPC } \\
\text { DOPA } \\
\text { DOPE } \\
\text { Monoolein }\end{array}$ & $\begin{array}{l}\text { Practically insoluble, the molecules swell to form } \\
\text { lyotropic liquid crystalline phases }{ }^{3}\end{array}$ & Molecules spread to form stable monolayers \\
\hline $\begin{array}{l}\text { Class IIIA }^{2} \\
\text { Sodium oleate } \\
\text { Potassium oleate } \\
\text { Oleoyl-lyso-PC }\end{array}$ & $\begin{array}{l}\text { Soluble, formation of micelles at high water } \\
\text { content above the } \mathrm{CMC}^{4} \text {; formation of liquid } \\
\text { crystalline phases }{ }^{3} \text { at low water content }\end{array}$ & $\begin{array}{l}\text { Molecules spread but form unstable monolayers } \\
\text { due to the solubility in water }\end{array}$ \\
\hline
\end{tabular}

${ }^{1}$ For chemical structures; see Figure 2. ${ }^{2}$ Polar lipids belonging to Class IIIB have the same behavior at the water-air interface like Class IIIA lipids and form micelles above the critical micellization concentration, CMC, but they do not form liquid crystals. ${ }^{3}$ Lyotropic liquid crystalline phases (also called mesophases) have a short-range disorder but some distinct order over larger distances, whereby the phase type depends on both water content and temperature $[42,43] .{ }^{4}$ Micellar solutions are isotropic solutions, characterized by disorder over short and long distances [42,43]. The reported critical micellization concentration $(C M C)$ of sodium oleate at $T=24^{\circ} \mathrm{C}$ in $\mathrm{D}_{2} \mathrm{O}$ is about $0.7 \mathrm{mM}$ [44]. The CMC of oleoyl-lyso-PC at $T=25^{\circ} \mathrm{C}(20 \mathrm{mM}$ HEPES buffer, $150 \mathrm{mM} \mathrm{NaCl}, p H=7.4)$ is about $5 \mu \mathrm{M}$ [45].

\subsection{Oleic Acid and Palmitic Acid}

Oleic acid (= cis-9-octadecenoic acid $=(9 Z)$-octadec-9-enoic acid) and palmitic acid (= hexadecanoic acid) belong to Class I of the polar lipids (Table 1 ). They differ in their melting temperatures: $13.4{ }^{\circ} \mathrm{C}\left(\alpha\right.$-form) and $16.2^{\circ} \mathrm{C}\left(\beta\right.$-form) for oleic acid and $63^{\circ} \mathrm{C}$ for palmitic acid [41]. Therefore, at room temperature $\left(T=25^{\circ} \mathrm{C}\right)$, the unsaturated oleic acid is a liquid and the saturated palmitic acid is a solid. The water-solubility of both fatty acids in monomeric (non-associated) form is very low and difficult to determine experimentally. Careful measurements at $T=37^{\circ} \mathrm{C}$ in $66 \mathrm{mM}$ sodium phosphate buffer at $p H=7.4$ indicated that for both fatty acids the solubility is below $1 \mu \mathrm{M}$ [46]. For palmitic acid the conclusion was that its water-solubility is even below $0.1 \mathrm{nM}$ [46].

If a drop of oleic acid is placed at the water-air interface, some of the oleic acid molecules will form a stable monolayer with the polar carboxylic acid head group being in contact with the water, and with the hydrophobic tail pointing towards the air. Only a few oleic acid molecules will be dissolved in the aqueous solution due to their low water-solubility [41]. Excess oleic acid molecules will form a separate, lighter, oily liquid phase on top of the water, with some of the oleic acid molecules expected to be localized at the oleic acid phase-water interface, with the polar head group in contact with water [41].

\subsection{Sodium Oleate/Water Mixtures}

The deprotonated form of oleic acid—oleate with $\mathrm{Na}^{+}$or $\mathrm{K}^{+}$as counter ion-behaves very differently from oleic acid when placed at the air-water interface. These soap molecules belong to Class IIIA of the polar lipids and form a monolayer which is very unstable (Table 1), since the oleate molecules are several orders of magnitude more soluble in water than oleic acid and self-assemble to form micelles at high water content or liquid crystalline phases at lower water content [47] (Figure 3a). Early investigations of the binary phase diagram of the sodium oleate-water and potassium oleate-water systems were carried out by Vold [48] and Mc Bain and Sierichs [49]. The critical micellization concentration (CMC) for sodium oleate in $\mathrm{D}_{2} \mathrm{O}$ was determined by Mahieu et al. (1991) at $T=24{ }^{\circ} \mathrm{C}$ and found to be $0.7 \mathrm{mM}(=0.02 \mathrm{wt} \%)$ [44]. Above the $C M C$ and above the melting temperature (about $23{ }^{\circ} \mathrm{C}$ at high water content), the micelles are spherical [47,50] (or cylindrical [51], Figure $3 b$ ) forming an isotropic $L_{1}$ phase, which transforms with increasing sodium oleate 
content into a concentrated system of entangled cylindrical micelles [47,52] (with observed viscoelastic behavior, denoted as $\mathrm{L}_{1}$ * in Figure $3 \mathrm{a}$ ), until a (normal) hexagonal liquid crystalline phase $\left(\mathrm{H}_{1}\right)$ is obtained at about $18 \mathrm{wt} \%$ sodium oleate (corresponding to $82 \mathrm{wt} \%$ water) (Figure 3a). The binary phase diagram in Figure 3a represents the thermodynamic equilibrium state, as obtained by Antunes et al. (2007) [47] after homogenization of samples prepared at the specific composition at $70{ }^{\circ} \mathrm{C}$, followed by storage at the desired temperature for 1 week before the aggregation state was analyzed. Obviously, changes in the chemical structure of the oleate molecules during such sample treatment and long-term storage should not occur. Otherwise, the determined phase diagram would not represent the situation for pure sodium oleate/water mixtures.
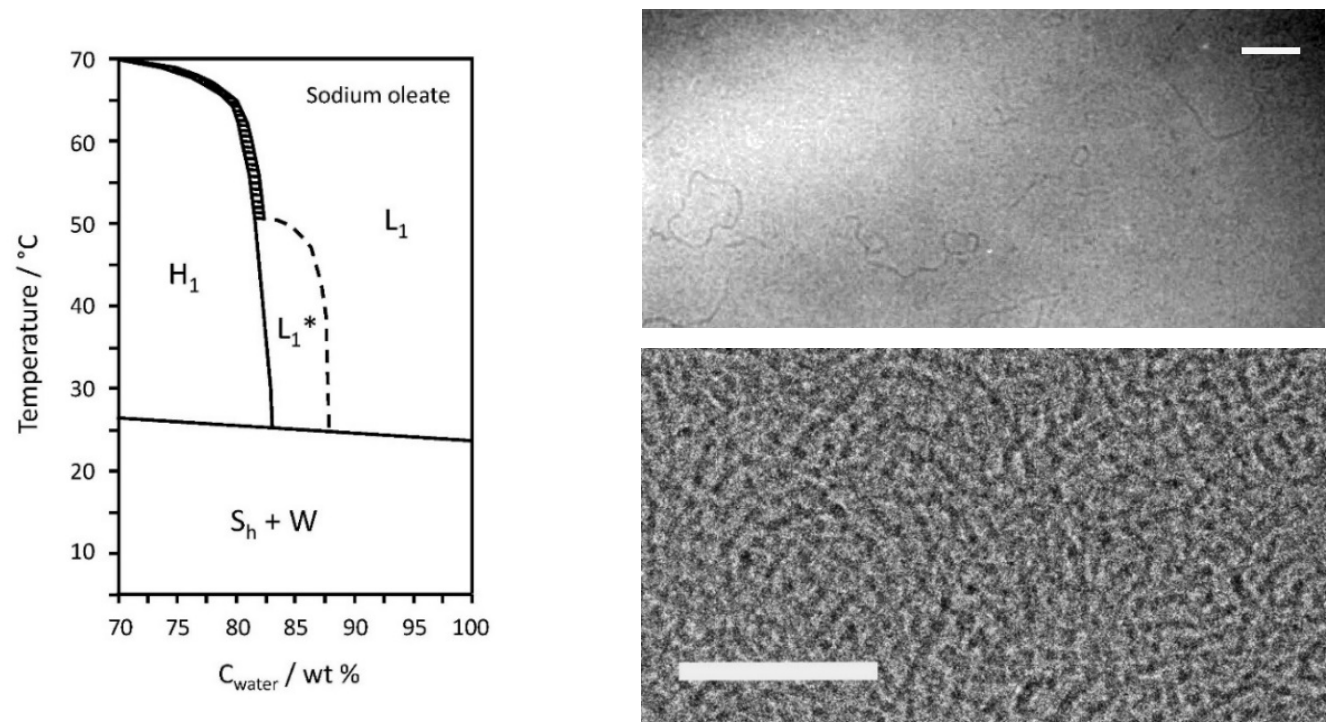

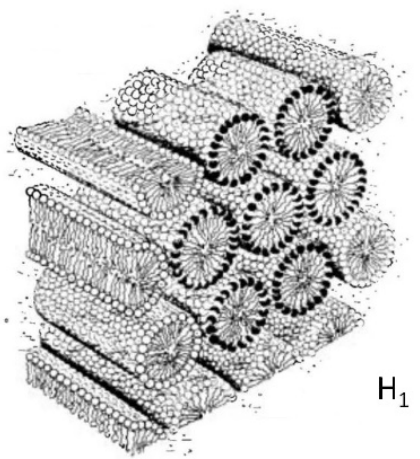

(a)

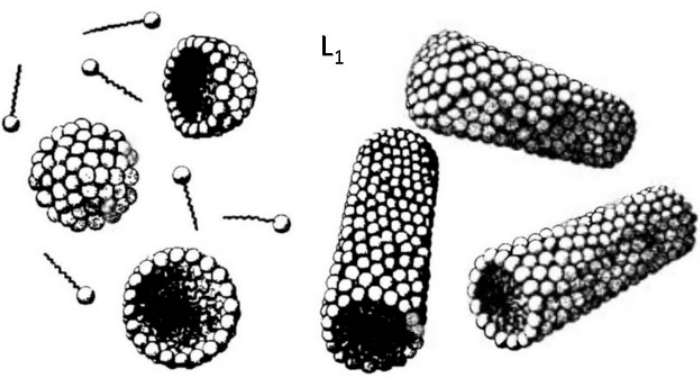

(b)

Figure 3. Aqueous dispersions of sodium oleate and water. (a) Top: Partial temperature-composition phase diagram, reproduced from Antunes et al. (2007) [47], as mirror image of the originally published diagram; $\mathrm{L}_{1}$ : micellar solution (consisting of spherical and flexible cylindrical, wormlike micelles); $\mathrm{L}_{1}{ }^{*}$, viscous micellar solution (consisting of entangled wormlike micelles [52]); $\mathrm{H}_{1}$, hexagonal liquid crystalline phase (consisting of long cylindrical micelles lying parallel to each other and arranged in a hexagonal lattice); $\left(S_{h}+W\right)$, hydrated oleate crystals plus water; dashed area, coexistence of $\mathrm{L}_{1}$ and $\mathrm{H}_{1}$. Bottom: Schematic representation of a hexagonal liquid crystalline phase, $\mathrm{H}_{1}$ [53]. (b) Top: Cryo-TEM image of a $3.6 \mathrm{mM}$ sodium oleate solution $\left(=0.11 \mathrm{wt} \%\right.$ ) at $p H=10.7$, quenched from $T=25^{\circ} \mathrm{C}$ (bar: $100 \mathrm{~nm}$ ), showing long cylindrical oleate micelles, reproduced from Edwards et al. (1995) [51]. Middle: Cryo-TEM image of a $0.45 \mathrm{M}$ sodium oleate dispersion $\left(=13 \mathrm{wt} \%\right.$ ), quenched from $T=25^{\circ} \mathrm{C}$ (bar: $50 \mathrm{~nm}$ ), showing mostly wormlike micelles, reproduced from Tatini et al. (2021) [52]. Bottom: Schematic representation of a spherical and cylindrical (worm-like) micelles [53]. Reproduced with permission from [47], Elsevier, 2007; from [53], Society of Cosmetic Chemists, 1968; from [51], American Chemical Society, 1995; and from [52], Elsevier, 2021. 
A recent cryo-TEM analysis confirmed the presence of flexible cylindrical, wormlike sodium oleate micelles at $13 \mathrm{wt} \%(=0.43 \mathrm{M})$ [52] (Figure 3b). The presence of $\mathrm{NaCl}$ or $\mathrm{KCl}$ (as low as $4 \mathrm{wt} \%$ ) results in significant changes in the structure and properties of the wormlike oleate micelles [52]. This illustrates that small changes of the composition of the aqueous solution can have dramatic effects on the aggregation behavior of anionic oleate due to electrostatic interactions. Similarly, addition of hydrophobic molecules is expected to have a significant influence on the aggregation behavior.

Overall, sodium oleate is a micelle-forming lipid. Oleate micelles form at high water content above the $C M C$. Other types of aggregates form at low water content as a result of interactions between the micelles if the concentration of oleate is increased. Considering amphiphiles in the aggregated state as geometrical objects that constitute the aggregates, then sodium oleate- at conditions where spherical micelles form-can be viewed as inverted cone with a relatively large optimal area per polar head, $a_{0}$, at the spherical micelle surface [18].

Defining a critical packing parameter, $p$, for an aggregate-forming amphiphile as

$$
p=\frac{v}{a_{0} \times l_{c}}
$$

with $v$ being the hydrophobic volume occupied by the hydrophobic chain $(\mathrm{s}), l_{\mathrm{c}}$ the critical length of the hydrophobic chain(s), the limit of length the chain(s) could be stretched, then spherical micelles form for $p \leq 1 / 3$, ellipsoidal micelles for $1 / 3<p<1 / 2$, and cylindrical or rod-like micelles for $p \approx 1 / 2$ [18]. This packing parameter concept is widely used for qualitatively explaining the aggregation behavior of natural amphiphilic lipids and synthetic surfactants in aqueous media. However, $p$ cannot be determined from the chemical structure of the amphiphiles, since $a_{0}$ corresponds to the optimal area per polar area in the aggregate, as a result of hydrophobic attractions between the hydrophobic part of the amphiphiles and hydrophilic, ionic or steric repulsions of the head groups in the aggregated state [18].

The packing parameter, $p$, for sodium oleate in sodium oleate/water mixtures depends on the sodium oleate concentration. At $T=30^{\circ} \mathrm{C}$ and $97 \mathrm{wt} \%$ water, $p \approx 1 / 3$ (spherical micelles) or $p \approx 1 / 2$ (cylindrical micelles); for $75 \mathrm{wt} \%$ water, $p \approx 1 / 2\left(\mathrm{H}_{1}\right.$ phase consisting of aligned cylindrical micelles) (Figure 3) [53]. This packing parameter concept is very general and can be applied to any other "conventional" aggregate-forming amphiphiles, as long as they are in a fluid state (as in the case of the interior of a micelle). Often, $p$ is used for discussing effects of amphiphiles in relatively dilute systems (water-rich mixtures).

\subsection{Sodium or Potassium Oleate/Oleic Acid/Water Mixtures}

Determining the ternary phase diagram of mixtures of oleic acid, sodium oleate, and water as a function of temperature is very demanding, i.e., it means an enormous amount of work. A large number of samples of different compositions need to be prepared and analyzed. Often, the elaboration of such ternary phase diagrams is limited to a selected region and temperatures of interest, and the results are reported as triangular diagram for a specific temperature (Figure 4). Depending on the number of samples that were prepared and analyzed, and depending on how reliable the state of a mixture of a defined composition can be determined, a ternary phase diagram may look simple with a lot of uncertainties and unexplored areas, or it may consist of a lot of detailed information about the number of phases and the phase types and structures. One general difficulty is to ascertain that the analyzed samples are at thermodynamic equilibrium and do not represent kinetically trapped, metastable states.

McBain and Stewart published a ternary phase diagram of the oleic acid/potassium oleate/water system for $T=25^{\circ} \mathrm{C}$ already in 1933 [54], and Small reported one for the oleic acid/sodium oleate/water system for $T=37^{\circ} \mathrm{C}$ in 1968 [55]. A more recent and also more detailed triangular diagram for the oleic acid/sodium oleate/water system is the one published by Engblom et al. (1995) for $T=20{ }^{\circ} \mathrm{C}$ [56] (Figure 4a). Along the 
water sodium oleate base line of the triangle (representing samples without oleic acid), the situation for sodium oleate/water mixtures at $T=20^{\circ} \mathrm{C}$ is shown. According to Figure 3 a the $\mathrm{L}_{1}$ and $\mathrm{H}_{1}$ phases should not be present at $T=20^{\circ} \mathrm{C}$, but both phases seem to exist in the presence of small amounts of oleic acid (Figure 4a). At increasing content of oleic acid, other phases form as well, notably a lamellar phase $\left(\mathrm{L}_{\alpha}\right.$, which exists in the samples as large multilamellar vesicles), an inverted hexagonal phase $\left(\mathrm{H}_{2}\right)$, and a cubic phase of unknown structure (Q). Close to the oleic acid corner, an isotropic solution of inverted micelles forms $\left(L_{2}\right)$. The center of the lamellar phase region $\left(L_{\alpha}\right)$, as an example, corresponds to a composition of $40 \mathrm{wt} \%$ sodium oleate, $20 \mathrm{wt} \%$ oleic acid, and $40 \mathrm{wt} \%$ water (Figure 4a). Areas within the triangle that are not specified or labeled either represent compositions where phase separation occurred (for example, two phases of different composition, macroscopically separated from each other, appearing as denser lower phase and as lighter upper phase) or were not investigated at all.

If water is replaced by a $0.15 \mathrm{M}$ aqueous $\mathrm{NaCl}$ solution ( $=0.9 \mathrm{wt} \%)$, the ternary phase diagram looks different from the one with pure water, as shown by Mele et al. (2018) for $T=25^{\circ} \mathrm{C}$ [57]. This clearly indicates the effect of salts present in the aqueous solution on the aggregation properties. The triangular diagram for oleic acid/sodium oleate $/ 0.15 \mathrm{M}$ $\mathrm{NaCl}$ is shown in Figure $4 \mathrm{~b}$. No normal micelles form (i.e., no $\mathrm{L}_{1}$ phase) and no $\mathrm{H}_{1}$ exists at $T=25^{\circ} \mathrm{C}$. This can be explained by the screening of the negative charges of the head group. Furthermore, the region of the pure lamellar phase (L $\alpha 1)$ is much smaller than in the absence of $\mathrm{NaCl}$, and two different types of lamellar phases were identified in the presence of $0.1 \mathrm{M} \mathrm{NaCl}$ (a large region where $\mathrm{L} \alpha 1$ and $\mathrm{L} \alpha 2$ coexist). In addition to the $\mathrm{H}_{2}$ and $\mathrm{L}_{2}$ phases, two cubic phases were identified, the inverse bicontinuous cubic phase Pn3m and the inverse micellar cubic phase Fd3m; see [58] for their structures.

Overall, the situation is quite complex, despite the simple chemical structure of the two amphiphiles (oleic acid and oleate). Nevertheless, one important finding in relation to the formation of vesicles is that aqueous mixtures of oleic acid and oleate arrange into bilayers (lamellae), depending on the amounts and ratio of oleic and oleate. Oleate molecules are present at high $\mathrm{pH}$ values $(\mathrm{pH}>9)$, and oleic acid dominates at low $\mathrm{pH}$ and forms a separate phase (see 2.2). From a geometric point of view, inverted cones (oleate) transform into cylinders with a packing parameter $p \approx 1$ (pair of oleate + oleic acid) if the $\mathrm{pH}$-value is decreased to $\mathrm{pH} \approx 8.5$. Therefore, the $\mathrm{pH}$ value, the total oleic acid + oleate concentration, and the composition of the aqueous solution (presence of sodium chloride or inorganic buffer species) determine whether a lamellar phase forms or whether it does not form $[52,59]$.

The $\mathrm{pH}$ dependency of the aggregation state of oleic acid in various aqueous media was investigated by a number of research groups; see, for example, [41,50,51,60-68]. The main focus of these studies was not on the determination of the conditions for the formation of a thermodynamically stable lamellar phase, but on the formation of dispersed "oleic acid vesicles" - also called "oleic acid/oleate vesicles" to emphasize that the vesicle membrane consists of oleic acid as well as oleate molecules (forming cylindrical dimers with $p \approx 1$ ). For the sake of simplicity, we will continue to call these vesicles "oleic acid vesicles". A dilute aqueous sample of oleic acid vesicles with a total concentration of oleic acid plus sodium oleate of $80 \mathrm{mM}$ (about $2.4 \mathrm{wt} \%$ ), for example, represents a kinetically stable dispersed state of the lamellar phase (Figure 5). This state can be obtained, for example, by strong mechanical mixing of a thermodynamically stable oleic acid/oleate lamellar phase that coexists with water or an aqueous solution (i.e., $L \alpha+$ water or $L \alpha+$ aqueous solution). Once such aqueous oleic acid vesicle dispersion is obtained and one would wait for long enough, the vesicle dispersion would separate back into the two phases upon reaching thermodynamic equilibrium ( $\mathrm{L} \alpha+$ water or $\mathrm{L} \alpha+$ aqueous solution). 

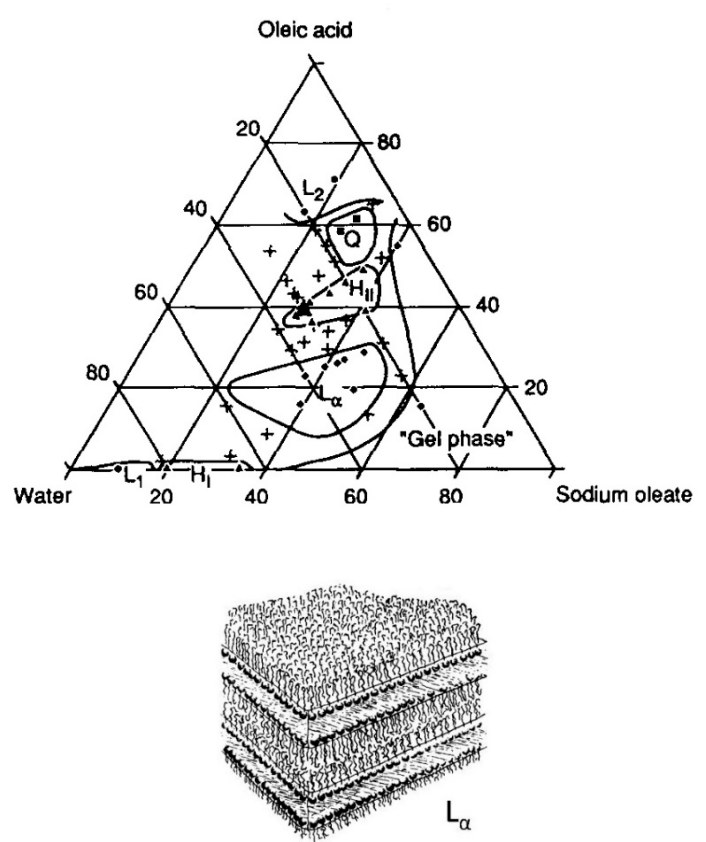

(a)

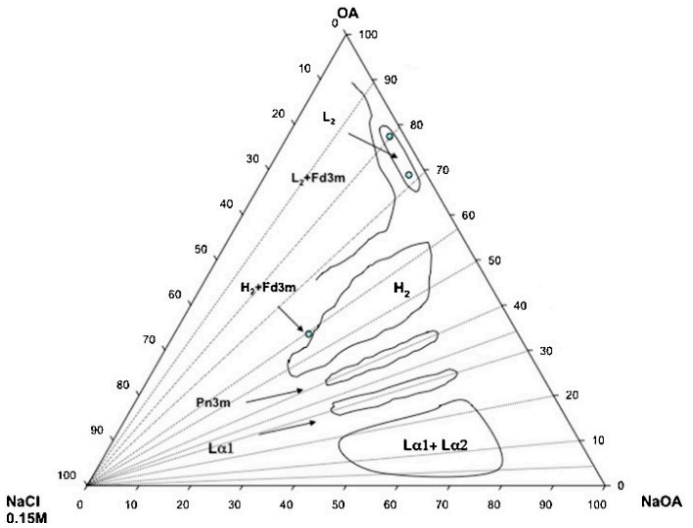

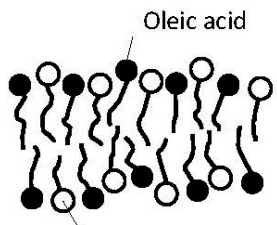

Oleate

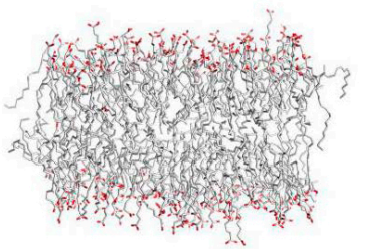

(b)

Figure 4. Aqueous mixtures of oleic acid and sodium oleate at $T=20$ or $25^{\circ} \mathrm{C}$. (a) Top: Ternary phase diagram of oleic acid/sodium oleate/water for $T=20^{\circ} \mathrm{C}$, as published by Engblom et al. (1995) [56]; $\mathrm{L}_{1}$, (normal) micellar phase; $\mathrm{H}_{\mathrm{I}}$, (normal) hexagonal phase; $\mathrm{L}_{\alpha}$, lamellar phase; $\mathrm{H}_{\mathrm{II}}$, inverse hexagonal phase; $\mathrm{Q}$, cubic phase; $\mathrm{L}_{2}$, inverse micellar phase. Bottom: Schematic representation of a lamellar phase $\left(\mathrm{L}_{\alpha}\right)$ [53]. (b) Top: Ternary phase diagram of oleic acid (OA)/sodium oleate (NaOA) / $0.1 \mathrm{M}$ aqueous $\mathrm{NaCl}$ at $T=25^{\circ} \mathrm{C}$, as published by Mele et al. (2018) [57]; $\mathrm{L} \alpha 1$ and L $\alpha$, two different lamellar phases; Pn3m, inverse bicontinuous cubic phase; $\mathrm{H}_{2}$, inverse hexagonal phase; Fd3m, inverse micellar cubic phase; $\mathrm{L}_{2}$, inverse micellar phase. In practice, the lamellar phase appears as multilamellar vesicles [57]. Self-closure into bilayers eliminates unfavorable interactions between the hydrophobic part and water at the edges of a planar bilayer. Bottom: Schematic representation of an oleic acid/oleate bilayer (1:1 molar ratio) with a snapshot from a molecular dynamics simulation, as published by Han (2013) [59]. Reproduced with permission from [56], Elsevier, 1995; from [53], Society of Cosmetic Chemists, 1968; from [57], Elsevier, 2018; and from [59], Elsevier, 2013.

The situation in the case of oleic acid + oleate mixtures is very similar to the case of vesicles formed from phospholipids like DOPC or POPC; see later Sections 2.10 and 2.11. All these vesicle dispersions are obtained by guided assembly procedures and usually are only kinetically stable dispersions, i.e., they do not represent thermodynamic phases. These vesicle dispersions can, however, be colloidally stable for several days or weeks. The cryoTEM images in Figure 5 demonstrate the existence of mainly unilamellar vesicles in aqueous oleic acid/sodium oleate dispersions, as obtained through guided assembly procedures at concentrations above the critical vesiculation concentration $(C V C)[51,63,65,68,69]$. The CVC is also known as "critical concentration for bilayer formation", CBC [70]. For oleic acid/oleate systems at $p H=8.5-9.9, C V C \sim 0.4-0.7 \mathrm{mM}[63]$.

\subsection{Monoolein/Water Mixtures}

Monoolein (=1-(cis-9-octadecenoyl)-rac-glycerol = glycerol monooleate) belongs to Class II of the polar lipids (Table 1). Although it has hemolytic properties, monoolein is a biodegradable and biocompatible amphiphile that generally is recognized as safe and useful for many applications, for example, in formulations for the delivery of drugs [71-74]. Monoolein/water systems were investigated intensively in the past [72,75-77]. Applications emerged from fundamental studies on the aggregation behavior of monoolein in water. The binary phase diagram published by Briggs et al. (1996) [76] is reproduced in Figure 6a. It shows the phase situations with increasing water content up to $50 \mathrm{wt} \%$ for temperatures between $T=20$ and $110^{\circ} \mathrm{C}$. In an extended version of this diagram published 
by Qiu and Caffrey (2000) [77], the temperature range was extended down to $T=-15^{\circ} \mathrm{C}$. As seen in Figure $6 \mathrm{a}$, the melting temperature of pure monoolein $\left(\mathrm{no}_{2} \mathrm{O}\right)$ is $36^{\circ} \mathrm{C}$, the temperature at which the lamellar crystalline phase (Lc) melts into a fluid isotropic phase (FI). The phases that are present at $T=25^{\circ} \mathrm{C}$ with increasing water content are the following: between $\sim 7$ and $\sim 19 \mathrm{wt} \%$ water, a lamellar liquid crystalline phase exists $\left(\mathrm{L}_{\alpha}\right)$; between $\sim 19$ and $\sim 22 \mathrm{wt} \%, \mathrm{~L}_{\alpha}$ coexists with the inverse bicontinuous cubic phase Ia3d [58,78]; between $\sim 22$ and $\sim 38 \mathrm{wt} \%$, Ia3d is present; between $\sim 38$ and $\sim 39 \mathrm{wt} \%$, Ia3d coexists with the inverse bicontinuous cubic phase Pn3m (see $[58,77,78]$ ); between $\sim 39$ and $\sim 42 \mathrm{wt} \%$, Pn $3 \mathrm{~m}$ is present; and above $\sim 42 \mathrm{wt} \%$ (up to more than $95 \mathrm{wt} \%$ water), Pn $3 \mathrm{~m}$ coexists with water $\left(\mathrm{Pn} 3 \mathrm{~m}+\mathrm{H}_{2} \mathrm{O}\right)$. In this large water-rich area of the phase diagram, the cubic phase Pn3m can be dispersed in water by strong mechanical agitation or sonication, for example, to form kinetically stable cubosomes, i.e., dispersed cubic phase as small particles. Usually, without stabilizers, these cubosome particles are not very stable (see Chapter 3). A cryo-TEM image of a sample consisting of $98 \mathrm{wt} \%$ water and $2 \mathrm{wt} \%$ monoolein, quenched from room temperature, is shown in Figure $6 b$ [79]. In this bicontinuous cubic phase, the amphiphiles are arranged in bilayers with balanced convex and concave curvatures, which results in an energetically minimal structure (the mean curvature at any point on the surface is zero) [80]. On average, the packing parameter of monoolein in Pn3m is $p=1$.
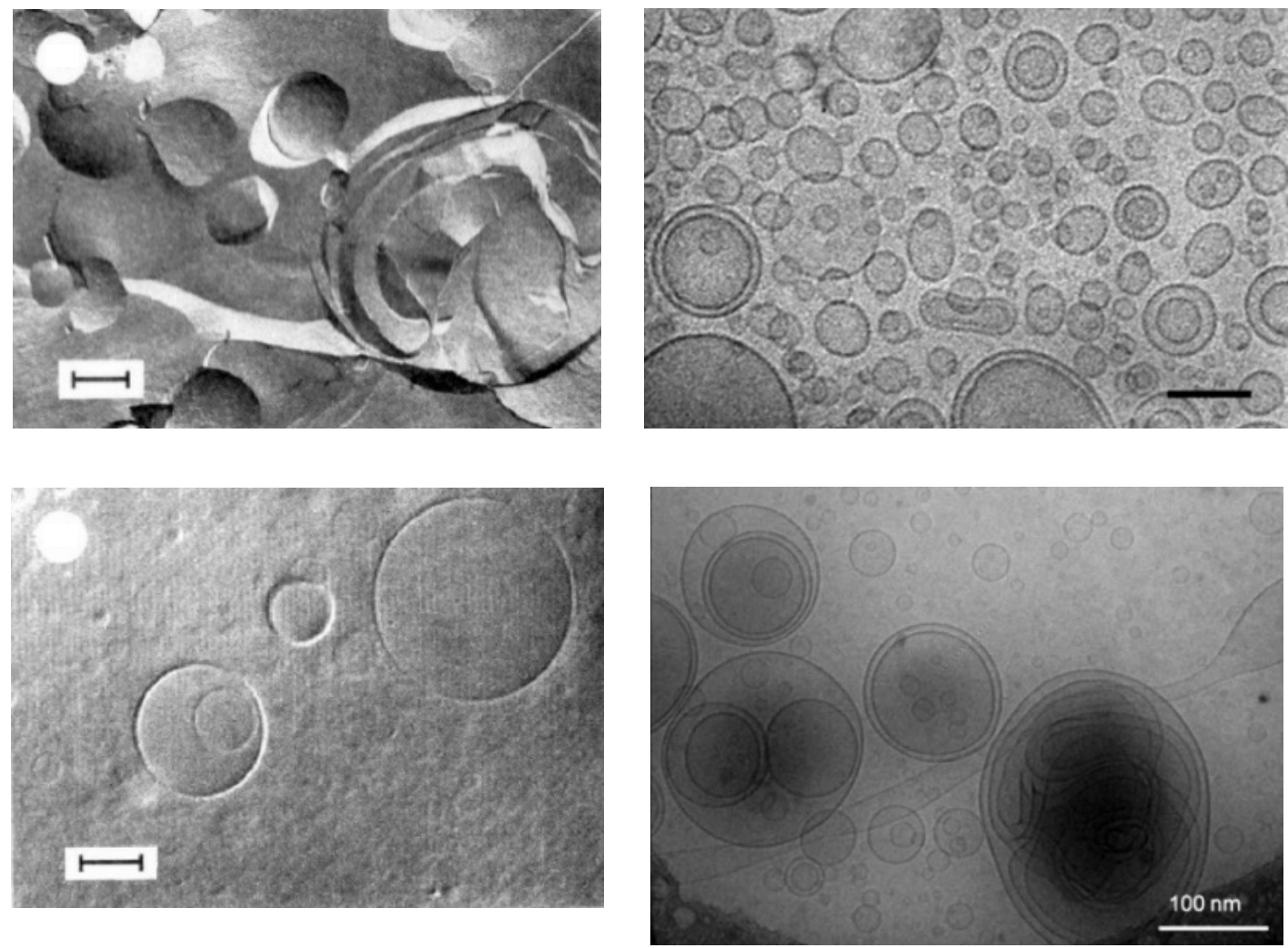

(a)

(b)

Figure 5. Electron and light microscopy visualizations of the formation of oleic acid vesicles in diulte aqueous media at $T=25^{\circ} \mathrm{C}$. (a) Freeze fracture electron microsopy image (top, bar: $200 \mathrm{~nm}$ ) and differential interference light microscopy image (bottom, bar: $10 \mu \mathrm{m}$ ) of spontaneously formed oleic acid vesicles at $p H=8.5$ (prepared from oleic acid at $20 \mathrm{mM}$ in $0.2 \mathrm{M}$ Bicine buffer) [63]. (b) Cryo-TEM images of oleic acid vesicles prepared either at $5 \mathrm{wt} \%(=177 \mathrm{mM})$ oleic acid at $p H=8.0$ (0.2 M Tris buffer using $\mathrm{NaOH}$ to adjust the $p H$ value, followed by probe sonication) (top, bar: $100 \mathrm{~nm}$ ) [69], or from an oleic acid sample analyzed at $p H=8.6$ (prepared from an acidic solution by addition of $\mathrm{NaOH}$ ) (bottom, bar: $100 \mathrm{~nm}$ ) [68]. Critical vesiculation concentration, $C V C$ (oleic acid + oleate, $p H=8.5-9.9) \sim 0.4-0.7 \mathrm{mM}$ [63]. Reproduced with permission from [63], American Chemical Society, 1994; from [69], Elsevier, 2006; and from [68], American Chemical Society, 2010. 


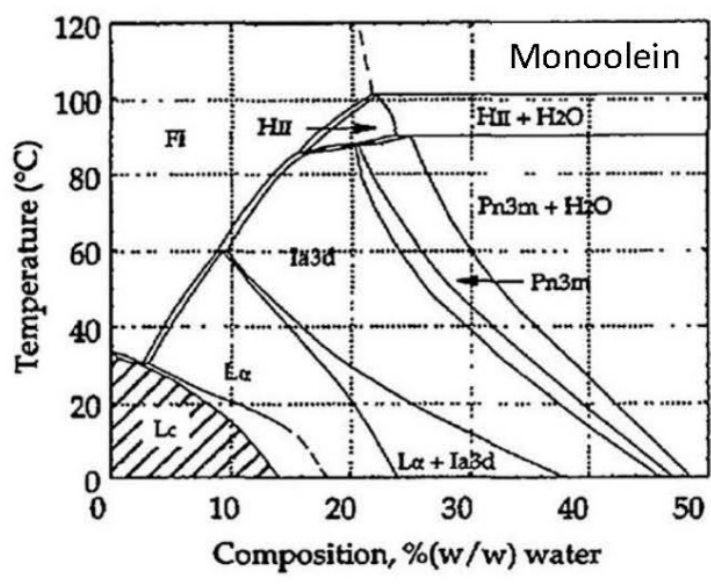

(a)
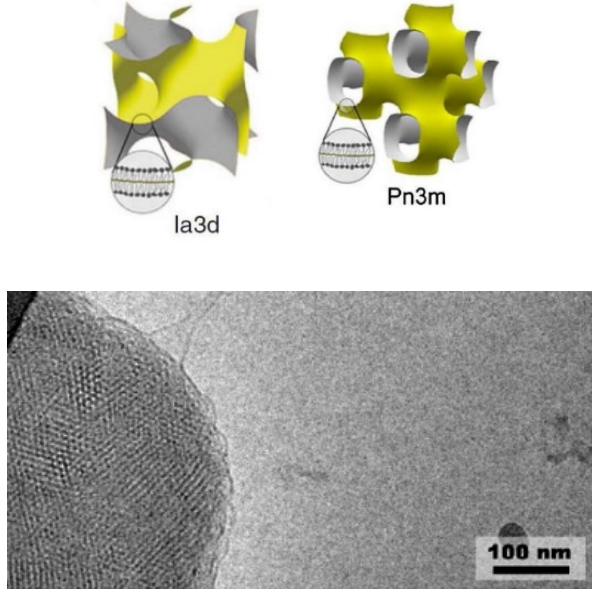

(b)

Figure 6. Monoolein cubic phases and cubosomes. (a) Binary monoolein/water phase diagram, indicating the localization of the inverse bicontinuous cubic phases Ia3d and Pn3m, the lamellar phase $\mathrm{L} \alpha$, the inverse hexagonal phase $\mathrm{H}_{\mathrm{II}}$, the fluid isotropic phase FI, the lamellar crystalline phase Lc, and the corresponding coexistence regions (two phases), as published by Briggs et al. (1996) [76]; (b) Top: Schematic representation of the arrangement of amphiphilic lipids in the cubic phases Ia3d and Pn3m; see Koynova and Tenchov (2013) [58] or Tenchov and Koynova (2017) [78]. The polar head groups of the amphiphiles point towards the internal water channels. Bottom: cryo-TEM image of dispersed monoolein cubic phase Pn3m in water ( $2 \mathrm{wt} \%$ monoolein, $98 \mathrm{wt} \% \mathrm{D}_{2} \mathrm{O}$; bar: $100 \mathrm{~nm}$ ) [79]. The structured area on the left-hand side is dispersed Pn3m, present as cubosome particles. Reproduced with permission from [76], EDP Sciences, 1996; from [78], Elsevier, 2017; and from [79], Elsevier, 2003.

\subsection{Monoolein/Sodium Oleate/Water Mixtures}

Aqueous mixtures of monoolein, sodium oleate, and water were investigated systematically. The triangular phase diagram published by Borné et al. (2001) for $T=25^{\circ} \mathrm{C}$ is shown in Figure 7a [81]. A similar diagram was also determined for $T=37^{\circ} \mathrm{C}$ [81]. One characteristic feature of both diagrams is the large region where a lamellar phase $\left(\mathrm{L}_{\alpha}\right)$ is present. A cryo-TEM image of this lamellar phase is shown in Figure $7 \mathrm{~b}($ top $)$ for $10 \mathrm{wt} \%$ monoolein, $5 \mathrm{wt} \%$ sodium oleate, and $85 \mathrm{wt} \%$ water [81]. The lamellar phase appears as multilamellar vesicles. To distinguish this lamellar phase from the lamellar phase obtained for the monoolein/water system (no oleate, $\mathrm{L}_{\alpha}$ in Figure $6 \mathrm{a}$, abbreviated as $\mathrm{L}_{\alpha 1}$ ), the monoolein/oleate/water lamellar phase is abbreviated as $\mathrm{L}_{\alpha 2}$ [81]. Unilamellar vesicles representing dispersed $\mathrm{L}_{\alpha 2}$ phase were found to be present in multiphase regions of the phase diagram shown in Figure 7a localized close to the water corner ( $>90 \mathrm{wt} \%$ water), in proximity to the region where the lamellar phase, $\mathrm{L}_{\alpha 2}$, was identified; see the cryo-TEM image of Figure $7 \mathrm{~b}$ (bottom) [79].

The monoolein/sodium oleate/water system is a nice example for illustrating how new aggregates can emerge when mixtures of amphiphiles are dispersed in water as compared to dispersions of the individual amphiphiles. This is very similar to the oleic acid/sodium oleate/water system; see Section 2.4. Neither monoolein alone nor sodium oleate alone aggregates into a lamellar phase in excess water. Only when they are present together does planar bilayer formation occur, which can be explained by a change in the packing parameter of the amphiphiles. Oleate packs as inverted cone-with a "large" polar head group-into spherical micelles at concentrations just above the $C M C(p \leq 1 / 3)$; see Section 2.3. The large head group area originates from the hydrated, negatively charged carboxylate, which results in head group repulsion when the oleate molecules assemble on the basis of hydrophobic interactions between the hydrophobic oleoyl chain. This polar head group repulsion is reduced if monoolein is present in the aggregate, selfassociating between the oleate molecules. This induces a change of the inverted cone of oleate into a cylindrical geometry which favors the formation of bilayers (with $p \approx 1$ for 
the oleate/monoolein pair). This is conceptually the same as what is happening in the oleic acid/sodium oleate/water system where oleic acid takes the role of monoolein.

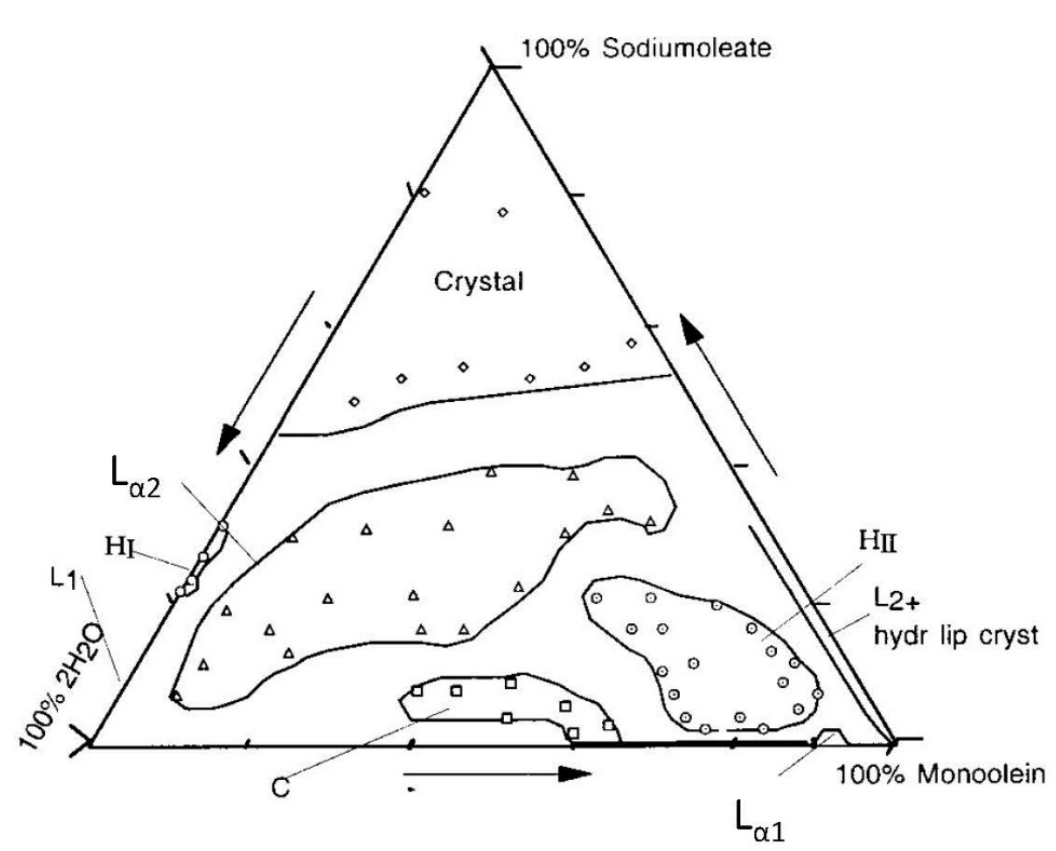

(a)
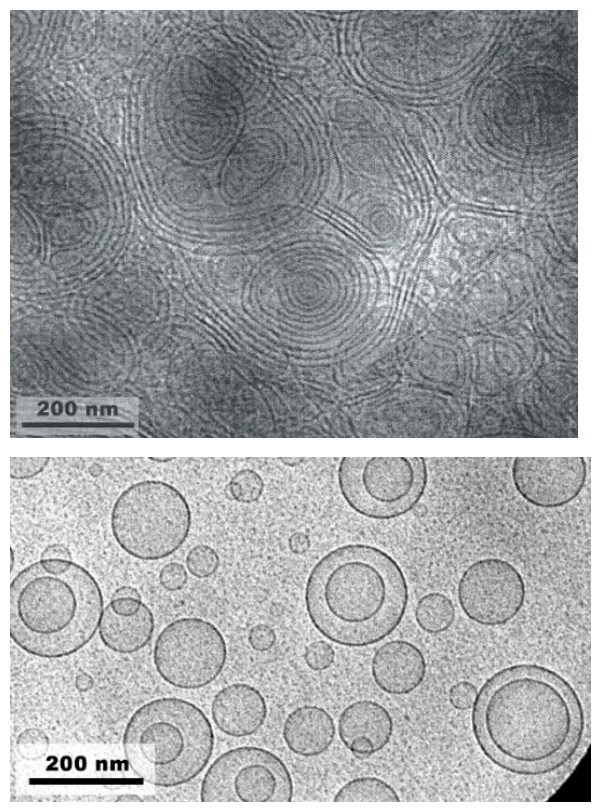

(b)

Figure 7. Aqueous mixtues of sodium oleate and monoolein. (a) Ternary monoolein/sodium oleate/water phase diagram for $T=25^{\circ} \mathrm{C}$, as presented by Borné et al. (2001) [81]. For the situations represented on the base lines between the water and the sodium oleate corner and between the water and the monoolein corner of the triangle, see Figures $3 a$ and $6 a$, respectively. The lamellar phase localized close to the monoolein corner is abbreviated as $\mathrm{L}_{\alpha 1}$. (b) Cryo-TEM images of the lamellar phase ( $\mathrm{L}_{\alpha}=\mathrm{L}_{\alpha 2}$, appearing as multilamellar vesicles), as present in a sample consisting of $10 \mathrm{wt} \%$ monoolein, $5 \mathrm{wt} \%$ sodium oleate, and $85 \mathrm{wt} \%$ water (top) [81]; and of dispersed vesicles present in a sample consisting of $0.2 \mathrm{wt} \%$ monoolein, $1.8 \mathrm{wt} \%$ sodium oleate, and $98 \mathrm{wt} \%$ water (bottom) [79]; both samples were quenched from $T=25^{\circ} \mathrm{C}$. Reproduced with permission from [81], American Chemical Society, 2001; and from [79], Elsevier, 2003.

\subsection{Monoolein/Oleic Acid/Water and Monoolein/Oleic Acid/Sodium Oleate/Water Mixtures}

As discussed above, there is a huge difference between the Class III polar lipid sodium oleate and the Class I polar lipid oleic acid in terms of water solubility and the ability to swell in water and, therefore, in terms of properties at the water-air interface (Table 1). With this, it is not surprising that the ternary monoolein/oleic acid/water phase diagram is very different from the ternary monoolein/sodium oleate/water phase diagram [81]. For the monoolein/oleic acid/water system there is no large lamellar phase region, as was found for the monoolein/sodium oleate/water system (Figure 7a). There is, however, an inverse hexagonal phase $\left(\mathrm{H}_{\mathrm{II}}\right)$ area (the packing parameter for the monoolein/oleic acid pair is $p>1$ ), although considerably smaller than in the case of the monoolein/ sodium oleate/water system. Moreover, the presence of an inverse micellar cubic phase of space group Fd3m was also identified, mainly coexisting with other phases [81].

The situation becomes complicated if aqueous mixtures of monoolein, oleic acid, and oleate are investigated. The ratio of oleic acid to oleate can be set by adjusting the $\mathrm{pH}$ value, often involving the use of buffer salts. The buffer salts themselves may also have an effect on the aggregation state and on the kinetic stability of dispersed phases. Moreover, it was demonstrated by Fong et al. (2020) [82] that with small amounts of the amphiphilic nonionic block copolymer Pluronic F127 (=Poloxamer 407) [83] dispersed micellar cubic phase Fd3m (cubosomes) can be stabilized (Figure 8a). In this latter work, it was also shown that by increasing the amount of oleate in the system (by increasing the $\mathrm{pH}$ value using phosphate-buffered saline, $\mathrm{PBS}$ ), the inverse micellar cubosome dispersion 
obtained at $p H=4.3$ (at low ionic strength) transforms into hexosomes, i.e., dispersed inverse hexagonal phase $\mathrm{H}_{2}$ at $p H \sim 7.4$ and $T=30^{\circ} \mathrm{C}$ [82]. Figure $8 \mathrm{~b}$ depicts this phase transformation as a consequence of a change of $p H$ value and salt content, which resulted in a change of the molecular packing [82].

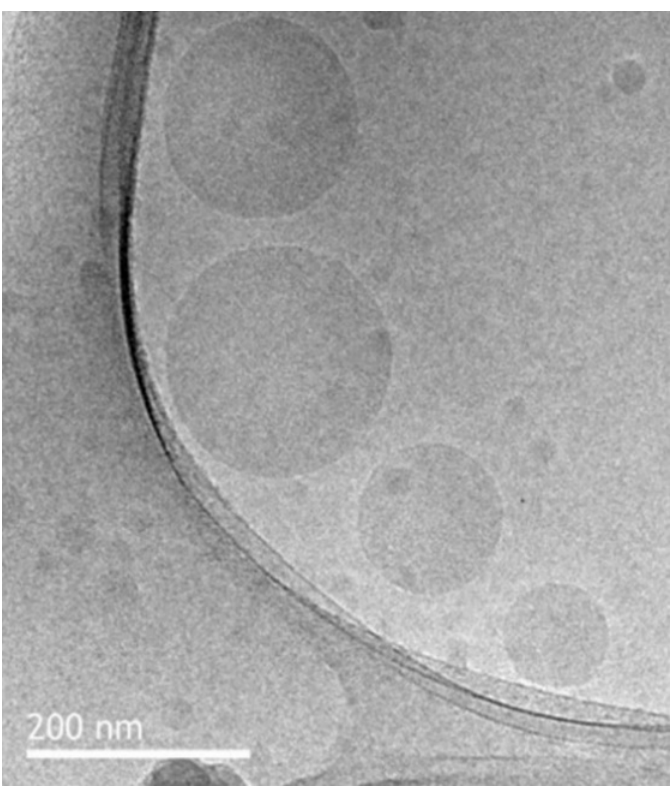

(a)

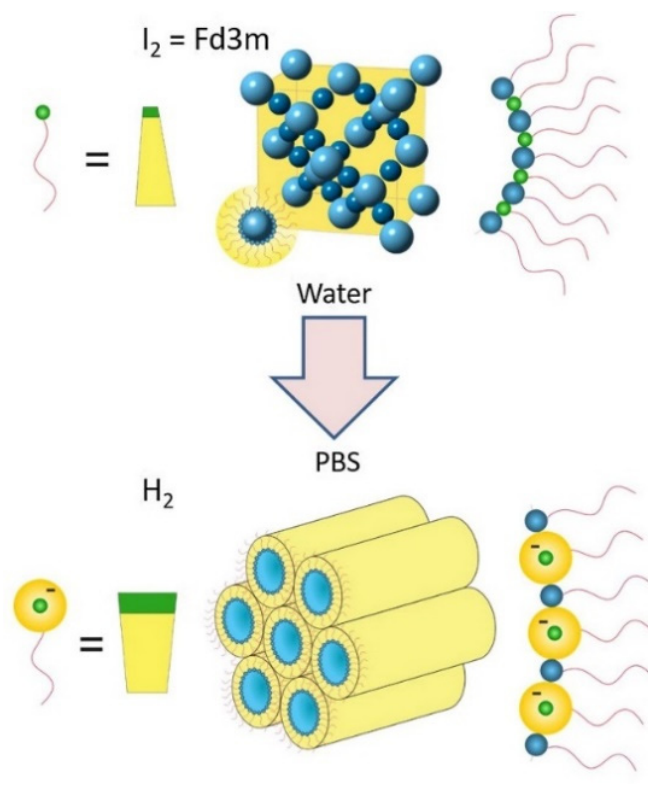

(b)

Figure 8. Cubosomes and hexosomes. (a) Cryo-TEM image of an aqueous dispersion of cubosomes consisting of the inverse micellar cubic phase Fd3m formed in the monoolein/oleic acid/water system at $T=30{ }^{\circ} \mathrm{C}$ at a monoolein/oleic acid weight ratio of 1:1, stabilized by the amphiphilic block copolymer Pluronic F127 (bar: 200 nm), as published by Fong et al. (2020) [82]. (b) Schematic representation of the effect of $p H$ and phosphate buffered saline (PBS) on the aggregation state of aqueous mixtures of monoolein, oleic acid (and oleate), and water at $T=30^{\circ} \mathrm{C}$ : dispersed inverse micellar cubic phase $\mathrm{Fd} 3 \mathrm{~m}$ (cubosomes) at $p H=4.3$ and low ionic strength (top); dispersed inverse hexagonal phase (hexosomes) at $p H \sim 7.4$ in the presence of PBS (bottom). The cubosome $\rightarrow$ hexosome transformation is explained by considering a change in the geometry of the molecular packing of the amphiphiles. The lipid with blue head group represents monoolein. The lipid with green head group represents either neutral oleic acid at low $\mathrm{pH}$ (small head group) or negatively charged oleate at high $p H$ (large head group) [82]. Reproduced with permission from [82], Elsevier, 2020.

\subsection{Diolein}

Like other diacylglycerols, 1,3- and 1,2-diolein belong to Class I of the polar lipids (Table 1). The water solubility of both isomers is very low and the molecules do not swell when brought in contact with water [41].

\subsection{Oleoyl-Lyso-PC/Water Mixtures}

The lysolecithin oleoyl-lyso-PC (=1-oleoyl-sn-glycero-3-phosphocholine = 1-oleoyl2-hydroxy-sn-glycero-3-phosphocholine) belongs to Class IIIA of the polar lipids, like sodium or potassium oleate (Table 1). Oleoyl-lyso-PC is a "micelle-forming amphiphile" $(p \sim 1 / 3)$. Therefore, like in the case of the sodium oleate/water system (Figure 3a), the binary oleoyl-lyso-PC/water phase diagram has an extended isotropic region consisting of (normal) micelles. They self-assemble at $T=25^{\circ} \mathrm{C}$ in water-rich mixtures, above about $75 \mathrm{wt} \%$ water, above the CMC; see Figure 9a. The diagram in Figure 9a was published by Marsh (2013) [84] on the basis of the one elaborated by Arvidson et al. (1985) [85]. In the diagram shown in Figure 9a, the region of the micellar solution (usually denoted as $\mathrm{L}_{1}$ ) is abbreviated as $\mathrm{M}_{\mathrm{I}}$. The presence of a normal hexagonal phase (consisting of packed cylindrical micelles, $\mathrm{H}_{\mathrm{I}}$ ) between 30 and $70 \mathrm{wt} \%$ water is a characteristic feature of this diagram, while a lamellar phase $\left(\mathrm{L}_{\alpha}\right)$ and a cubic phase $\left(\mathrm{Q}_{\mathrm{I}}\right)$ exist at low water content. 
1-oleoyl-2-lyso-sn-glycero-3-phosphocholine 1-(18:1c $\left.\Delta^{9}\right)-2-(0: 0) \operatorname{PtdCho}$

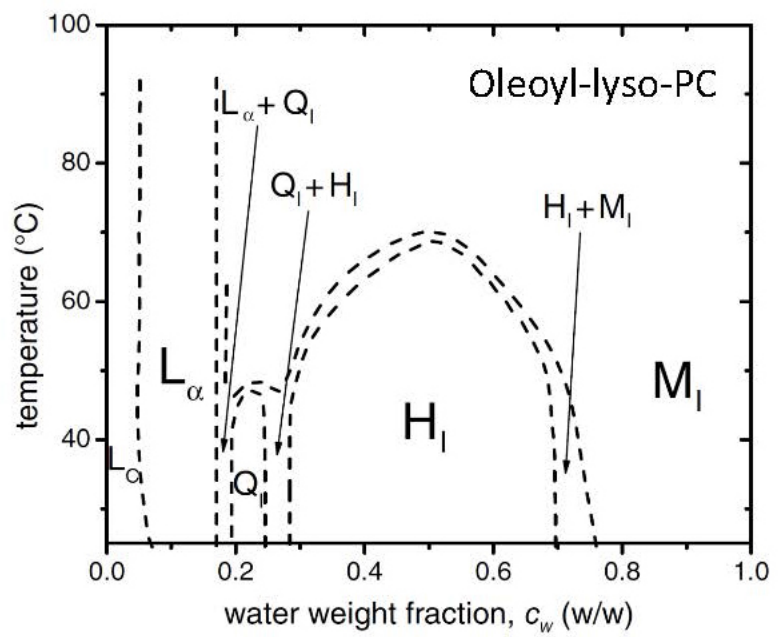

(a)
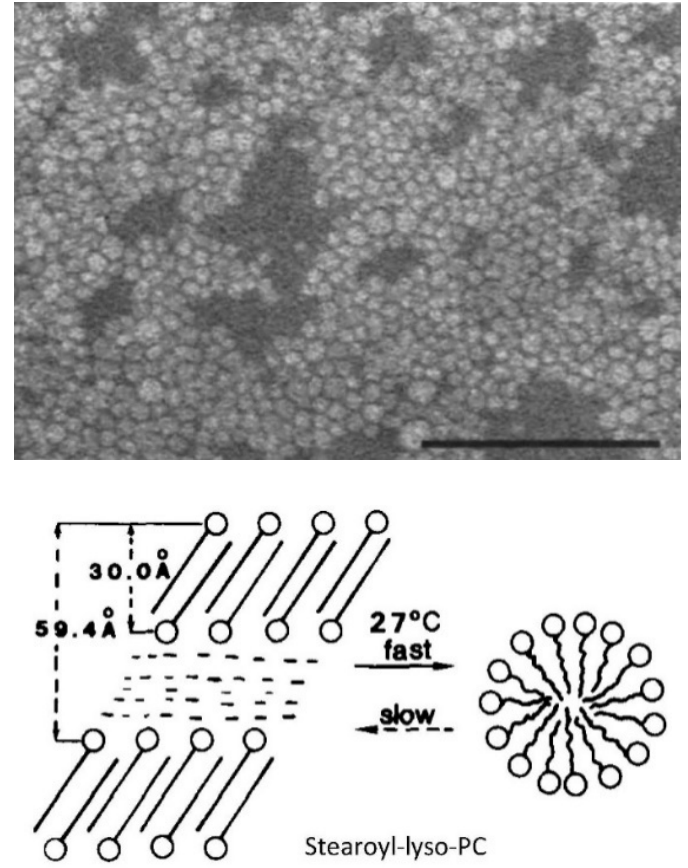

(b)

Figure 9. Aqueous solutions and dispersions of oleoyl-lyso-PC (or stearoyl-lyso-PC). (a) Binary oleoyl-lyso-PC/water phase diagram, as published by Marsh (2013) [84], a mirror image of the diagram originally published by Arvidson et al. (1985) [85]. Regions of the isotropic micellar phase (usually denoted by $\mathrm{L}_{1}$ ) are labeled with $\mathrm{M}_{\mathrm{I}}$, of the hexagonal phase with $\mathrm{H}_{\mathrm{I}}$, and of the cubic phase with $\mathrm{Q}_{\mathrm{I}}$. "1-oleoyl-2-lyso-sn-glycero-3-phosphocholine" is another description for oleoyl-lyso-PC. A water weight fraction of 0.8 , for example, means $80 \mathrm{wt} \%$ water. (b) Top: A micellar solution of $10 \mathrm{mM}(\sim 0.5 \mathrm{wt} \%)$ egg lyso-PC (obtained from egg yolk phosphatidylcholine after treatment with phospholipase $\mathrm{A}_{2}$ to remove the acyl chain in position sn-2 in the PC molecules), prepared in physiological saline, was analyzed by negative staining transmission electron microscopy in the dry state at room temperature (bar: $100 \mathrm{~nm}$; stained with sodium phosphotungstate); see Inoue et al. (1977) [86]. The presence of spherical micelles (with a fluid core) is evident from the electron micrograph. Bottom: Schematic representation of the temperature-dependent aggregation behavior of dilute aqueous dispersions of stearyol-lyso-PC. Stearoyl-lyso-PC forms spherical micelles at elevated temperature and "interdigitated" bilayer structures at low temperature. In the interdigitated bilayer state, the stearoyl chains interpenetrate; see Mattai and Shipley (1989) [87]. Reproduced from [84], copyright (C) Taylor and Francis, 2013; from [86], copyright @ Oxford University Press, 1977; and with permission from [87], Elsevier, 1986.

The $C M C$ value of oleoyl-lyso-PC was determined to about $5 \mu \mathrm{M}$ in HEPES buffer $(20 \mathrm{mM}, 150 \mathrm{mM} \mathrm{NaCl})$ at $p H=7.4$ and $T=25^{\circ} \mathrm{C}$ by Bergstrand and Edwards (2001) [45]. Micelles at a concentration of $1.34 \mathrm{mM}$ oleoyl-lyso-PC were found to be more or less spherical $(p \leq 1 / 3)$ with an average aggregation number of 142 [45]. An electron micrograph of micelles formed in aqueous solution by the related lysolecitin mixture from egg yolk is shown in Figure 9b (top) [86].

Depending on the melting temperature of the lysolecitin, a transformation of the micelles into interdigitated, crystalline lamellar bilayers occurs below the melting temperature, as illustrated in Figure $9 \mathrm{~b}$ (bottom) on the basis of a detailed investigation of stearoyl-lysoPC, i.e., a lysolecithin consisting of a hydrophobic chain of 18 carbon atoms without any double bonds [87]. Above $40 \mathrm{wt} \%$ water, the melting temperature of stearoyl-lyso-PC is $T_{\mathrm{m}}=27^{\circ} \mathrm{C}[87]$.

\subsection{DOPC/Water Mixtures}

DOPC belongs to Class II of the polar lipids (Table 1). It is a typical "bilayer-forming amphiphile" ( $p \approx 1)[88,89]$. This is evident from the binary phase diagram determined by Bergenståhl and Stenius (1987) [90], redrawn in a compilation of phase diagrams by 
Marsh (2013) [84]; see Figure 10a. Apart from an inverse cubic phase ( $\left.\mathrm{Q}_{\text {II }}\right)$ at low water content between $T=60$ and $120^{\circ} \mathrm{C}$ and an inverse hexagonal phase $\left(\mathrm{H}_{\mathrm{II}}\right)$ at even higher temperature, a lamellar phase $\left(\mathrm{L}_{\alpha}\right)$ exists between $\sim 10 \mathrm{wt} \%$ water (at $T=25^{\circ} \mathrm{C}$ ) and $\sim 42 \mathrm{wt} \%$ water. Above $\sim 42 \mathrm{wt} \%$ water, this lamellar phase is in equilibrium with water, forming a two-phase system $\left(\mathrm{L}_{\alpha}+\right.$ water). Dispersing the lamellar phase (multilamellar vesicles, MLVs, in reality) in excess water results in the formation of dispersed vesicles, i.e., self-closed bilayers, often with diameters above $500 \mathrm{~nm}$. These vesicles are only kinetically stable and will turn back to two phases after prolonged storage. Depending on how the lamellar phase is dispersed (see Chapter 4), the vesicles might be mainly unilamellar (see as an example the cryo-TEM image in Figure 10b [91]), and the vesicle dispersion may be colloidally stable for a long time without aggregation and eventually fusion into multilamellar vesicles and without macroscopic phase separation.

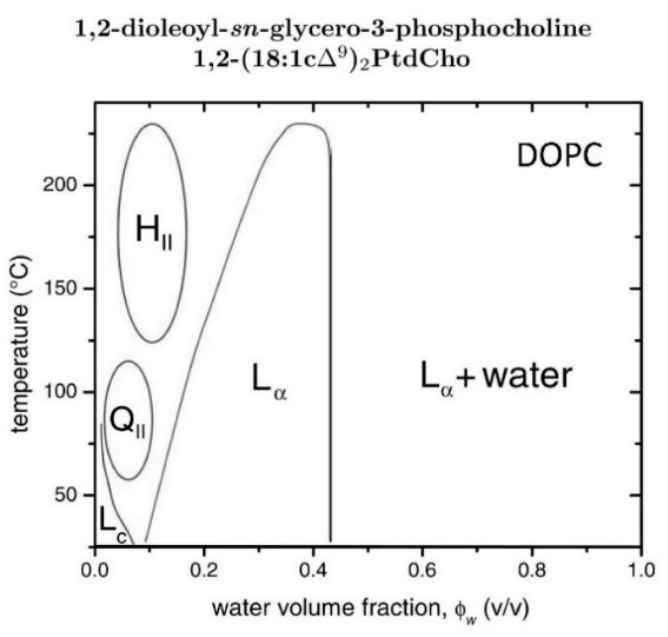

(a)

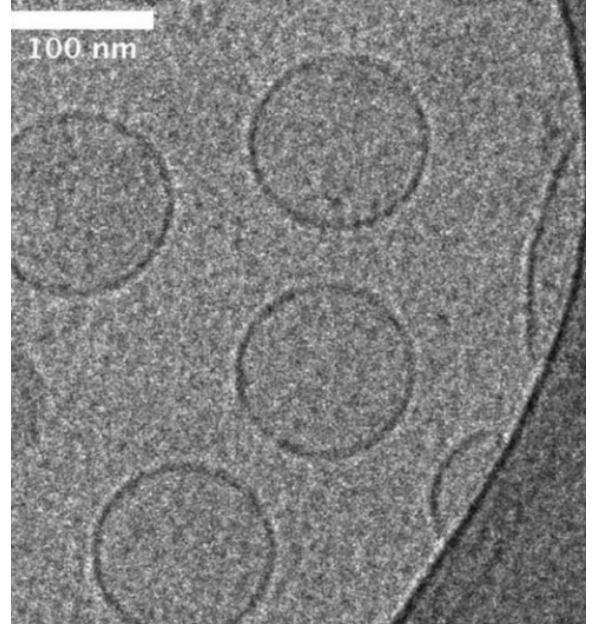

(b)

Figure 10. Aqueous dispersions of DOPC. (a) Binary DOPC/water phase diagram, published by Marsh (2013) [84], drawn on the basis of the phase diagram elaborated by Bergenståhl and Stenius (1987) [90]. L $\alpha$, lamellar phase; L $\alpha+$ water, two coexisting phases; Lc, lamellar crystals; $\mathrm{Q}_{\mathrm{II}}$, inverse cubic phase, $\mathrm{H}_{\mathrm{II}}$, inverse hexagonal phase. (b) Example of a cryo-TEM image of a dispersion of unilamellar DOPC vesicles $(0.1 \mathrm{wt} \%$ in water $(=1.3 \mathrm{mM}$; bar: $100 \mathrm{~nm})$, prepared with the polycarbonate membrane extrusion method (see Chapter 4), using for the final extrusions 100 nm pore membranes; see Hoffmann et al. (2014) [91]. Reproduced from [84], copyright (C) Taylor and Francis, 2013; and with permission from [91], Royal Society of Chemistry, 2014.

\subsection{Egg PC/Water or POPC/Water Mixtures}

Egg PC is a mixture of phosphatidylcholines isolated from chicken egg yolk. All molecules have the same polar head group (zwitterionic sn-glycero-3-phosphocholine). Variations among the molecules exist in the type of fatty acid chains esterified to the two hydroxyl groups at positions sn-1 and sn-2 of glycerol; see Table 2. In Table 2, data for soybean PC (a mixture of extracted phosphatidylcholines from soybean) are also included. In the case of egg PC, the majority of the fatty acyl chains are palmitoyl (C16:0) and oleoyl (C18:1) chains, while in the case of soybean PC, linoleoyl (C18:2) chains clearly dominate $[92,93]$. The binary eggPC/water phase diagram originally published by Small (1967) [94] and redrawn by Marsh (2013) [84] is shown in Figure 11a. In Figure 11b (top), the famous electron micrograph of a multilamellar dispersion of egg PC in water is shown, as published by Bangham and Horne (1964) [95], together with a cryo-TEM image of a dispersion of egg PC vesicles prepared by the detergent dialysis method (bottom) [96]. The pioneering work of Bangham is the beginning of the research on liposomes (lipid vesicles), originally also called “Banghasomes" [16,17]. 
Table 2. Some of the results obtained from the determination of the acyl chain composition in egg PC and soybean $\mathrm{PC}^{1}$.

\begin{tabular}{ccc}
\hline Acyl Chain (Fatty Acid) ${ }^{\mathbf{2}}$ & Egg PC (\%) $^{\mathbf{3}}$ & Soybean PC (\%) $^{\mathbf{3}}$ \\
\hline C16:0 (palmitic acid) & $35.0(37.0 \pm 0.07)$ & $11.2(13.6 \pm 0.06)$ \\
C18:0 (stearic acid) & $13.4(14.1 \pm 0.03)$ & $11.9(3.7 \pm 0.02)$ \\
C18:1 (oleic acid) & $30.4(22.1 \pm 0.05)$ & $8.6(10.6 \pm 0.06)$ \\
C18:2 (linoleic acid) & $18.0(21.2 \pm 0.07)$ & $58.665 .9 \pm 0.09)$ \\
C18:3 (linolenic acid) & - & $9.9(6.2 \pm 0.04)$ \\
C20:4 (arachidonic acid) & $3.2(3.9 \pm 0.1)$ & - \\
\hline
\end{tabular}

${ }^{1}$ In chemistry, biochemistry and biology, egg (or soybean) PC is also called egg (or soybean) lecithin. In food technology, however, a "lecithin" consists of a mixture of different phospholipids (including PC); see Section 6.4 . ${ }^{2}$ C16:0 means 16 C-atoms, no double bond; C18:1 means $18 \mathrm{C}$-atoms, one double bond with cis-configuration. The position of the double bonds is determined by the biosynthesis and has a big effect on the melting temperature. For oleic acid, for example, the double bond is between carbon atoms 9 and 10 (C-9 and C-10), with the carbonyl C-atom being C-1. ${ }^{3}$ First values from Palacios and Wang (2005) [92]; values in parenthesis from Kiełbowicz et al. (2012) [93]. For the positional distribution (sn-1 and $s n-2)$, see Kiełbowicz et al. (2012) [93].

egg phosphatidylcholine

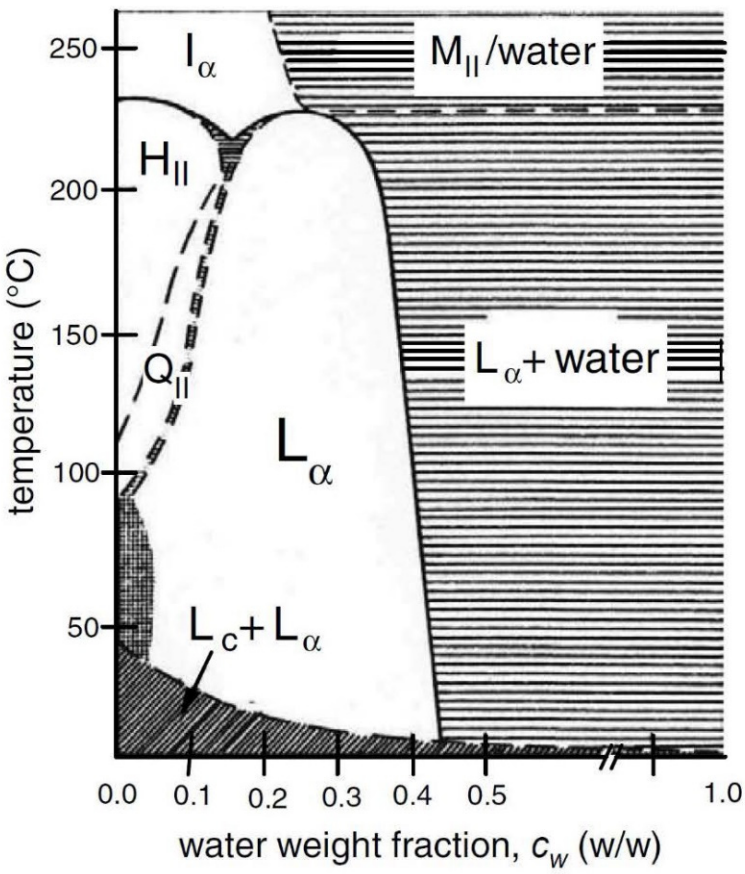

(a)
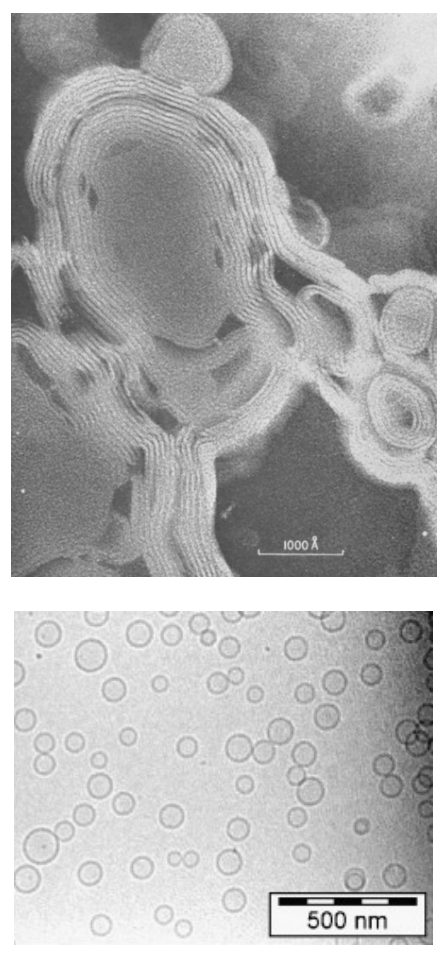

(b)

Figure 11. Aqueous dispersions of egg PC. (a) Binary egg PC/water phase diagram, published by Marsh (2013) [84], originally elaborated by Small (1967) [94]. $\mathrm{I}_{\alpha}$, a fluid isotropic phase; $\mathrm{M}_{\mathrm{II}}$, inverse micellar phase. (b) Top: Aqueous multilamellar egg PC dispersion (0.5 wt \% in water, $\sim 6.5 \mathrm{mM}$ ), analyzed by negative staining transmission electron microscopy in the dry state at room temperature (bar: $100 \mathrm{~nm}$; stained with potassium phosphotungstate); see Bangham and Horne (1964) [95]. White areas are occupied by the hydrophobic part of the egg PC lipids (no phosphotungstate), allowing the electron beam to pass through the sample to reach a beam-sensitive film; in the dark area, phosphotungstate is present so that the electron beam is reflected at the heavy atoms. Bottom: Example of a cryo-TEM image of unilamellar egg PC vesicles ( $<20 \mathrm{mM}$ in $5 \mathrm{mM}$ phosphate buffer, $p H=7.0$; bar: $500 \mathrm{~nm}$ ) prepared with the "detergent removal method" (see Chapter 4), as published by Holzer et al. (2009) [96]. Reproduced from [84], copyright ( Taylor and Francis, 2013; and with permission from [95], Elsevier, 1964; and from [96], Elsevier, 2009. 
The egg PC/water phase diagram is very similar to the DOPC/water phase diagram, and both diagrams are very similar to the soybean PC/water phase diagram published by Bergenståhl and Fontell (1983) [97]. In all three cases, there is a large region of lamellar phase $\left(L_{\alpha}\right)$, and at high water content, $L_{\alpha}$ coexists with water. This is the region where vesicle dispersions can be prepared easily by one of the different procedures that have been developed (see Chapter 4).

Although many basic studies on POPC/water mixtures were carried out, e.g., [98,99], a complete binary POPC/water phase diagram was not (yet) determined. It is, however, clear, that such diagram must be very similar to the ones of the DOPC/water or egg PC/water system shown in Figures 10 and 11. POPC is an "average" component of egg PC consisting of a palmitoyl and an oleoyl chain in positions sn-1 and sn-2, respectively [100] (Figure 2). Phosphatidylcholines with two long acyl chains (unsaturated or saturated) always form a two-phase system at high water content $\left(\mathrm{L}_{\alpha}+\right.$ water $)$ above the chain melting temperature (also known as main phase transition temperature, $T_{\mathrm{m}}$; see Section 2.12 ), and an $\mathrm{L}_{\alpha}$ phase between about 10 and $40 \mathrm{wt} \%$ water. These amphiphiles are perfect bilayer-forming lipids $(p \approx 1)$. One of the key differences between the different long chain phosphatidylcholines are their $\mathrm{T}_{\mathrm{m}}$ values. For DOPC, egg PC, soybean PC, and POPC, the $\mathrm{T}_{\mathrm{m}}$ values are all below $0{ }^{\circ} \mathrm{C}$. In the presence of excess water, the reported $\mathrm{T}_{\mathrm{m}}$ values are $-18.3 \pm 3.6^{\circ} \mathrm{C}$ (DOPC [101]), $-5.8 \pm 6.5^{\circ} \mathrm{C}\left(\operatorname{egg} \mathrm{PC}\right.$ [101]), between $-25^{\circ} \mathrm{C}$ and $-11^{\circ} \mathrm{C}$ (soybean PC [102]), and $-2.5 \pm 2.4{ }^{\circ} \mathrm{C}$ (POPC [101]). Above $T_{\mathrm{m}}$, the bilayers constituting the vesicle membrane are in a fluid, liquid-crystalline state $\left(\mathrm{L}_{\alpha}\right)$. Below $T_{\mathrm{m}}$, the molecules are in a crystalline-like state, as illustrated for the DPPC/water system in Section 2.12. For DOPC, for example, the low $T_{\mathrm{m}}$ value is not only due to the presence of a cis-double bond in the two acyl chains but also due to the position of these cis-double bonds, between C-9 and C-10; see Table 2. For a related PC molecule with a cis-double bond in both acyl chains between C-3 and C-4, $T_{\mathrm{m}} \sim 35^{\circ} \mathrm{C}[103,104]$.

\subsection{DPPC/Water Mixtures}

The binary DPPC/water phase diagram is very similar to the DOPC/water or egg $\mathrm{PC} /$ water phase diagrams. However, the main phase transition temperature of DPPC at high water content is $T_{\mathrm{m}}(\mathrm{DPPC})=41.3 \pm 1.8^{\circ} \mathrm{C}$ [101], which is considerably higher than $T_{\mathrm{m}}$ (DOPC) or $T_{\mathrm{m}}$ (egg PC); see Section 2.11. The consequence is that the fluid (liquid crystalline) lamellar phase $\left(\mathrm{L}_{\alpha}\right)$ exists at elevated temperature only, above $T_{\mathrm{m}} \sim 41{ }^{\circ} \mathrm{C}$ and above about $20 \mathrm{wt} \% \mathrm{H}_{2} \mathrm{O}$; see Figure 12a (top). This lamellar fluid ("liquid-crystalline") state of the amphiphilic lipids is also called "liquid disordered state" $\left(l_{\mathrm{d}}\right)[105,106]$.

Below $T_{\mathrm{m}}$, the DPPC molecules form bilayers that are crystalline-like, more ordered, less fluid than above $T_{\mathrm{m}}$. They are in a "solid-ordered state", $s_{\mathrm{o}}[105,107]$. Above $\sim 30-40 \mathrm{wt} \%$ water, the liquid disordered $\mathrm{L}_{\alpha}$ phase coexists with water above $T_{\mathrm{m}}$, while the solid ordered states which coexist with water below $T_{\mathrm{m}}$ are the ripple phase $\left(\mathrm{P}_{\beta^{\prime}}\right)$ and the $\mathrm{L}_{\beta^{\prime}}$ phase (also known as "gel phase") $[107,108]$. The prime (') indicates that the DPPC molecules are tilted with respect to the normal of the bilayer. Mechanical treatments of dilute aqueous PC dispersions have to be carried out at $T>T_{\mathrm{m}}$ in order to make it easier for the amphiphiles to reorganize according to the applied mechanical ("guiding") force; see Chapter 4. This means that for DPPC, such mechanical treatment should be done at a temperature which is clearly above room temperature. This is true for any other aqueous dispersions of lipids with high $T_{\mathrm{m}}$. Cryo-TEM images of DPPC vesicle dispersions are shown in Figure 12b [109-111], whereby the samples prepared were frozen from either $T>T_{\mathrm{m}}$ or $T<T_{\mathrm{m}}$. In the latter case, facetted vesicles are often observed, indicating the presence of rigid domains within the vesicle membrane; see also [112]. 

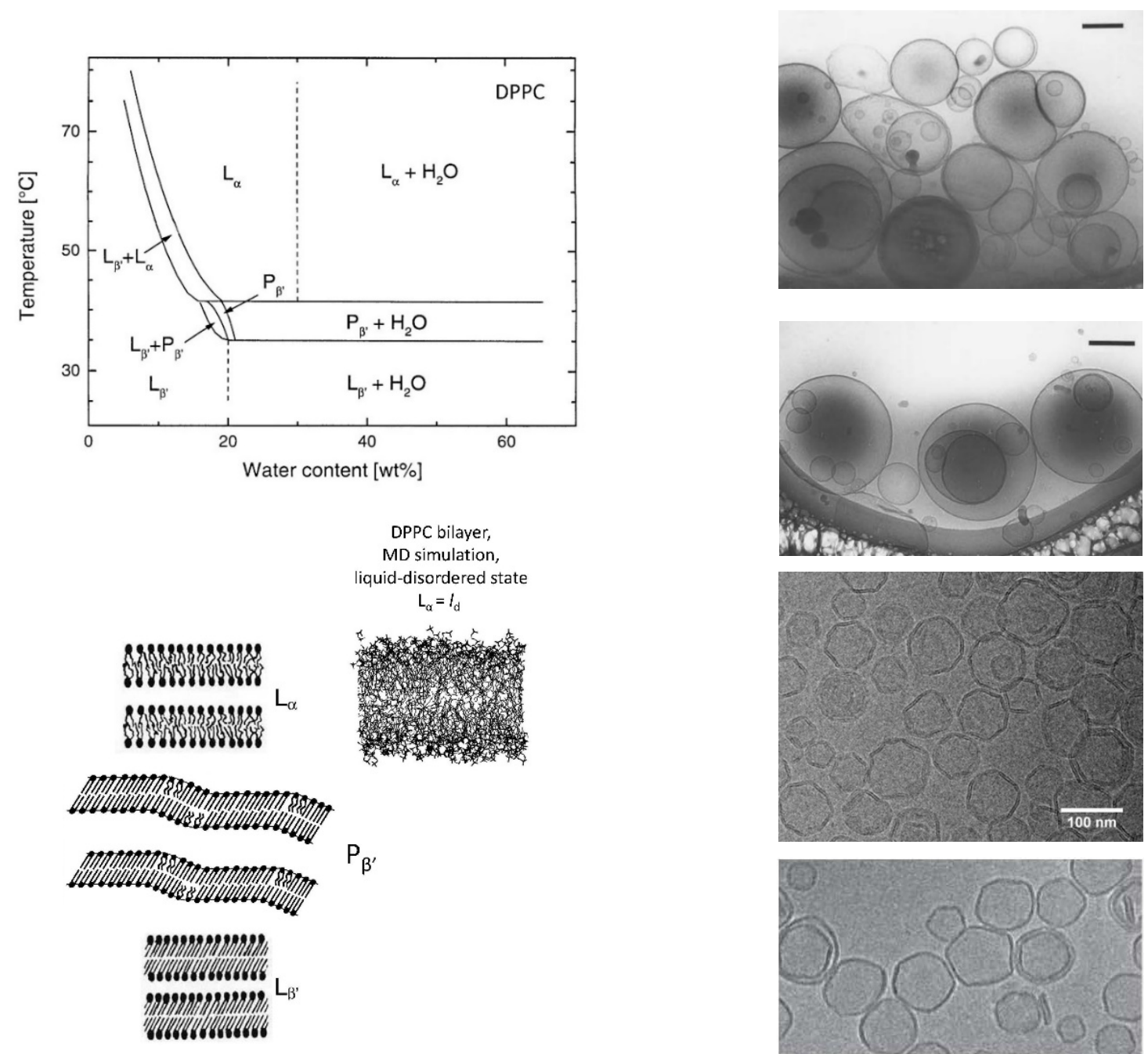

(a)
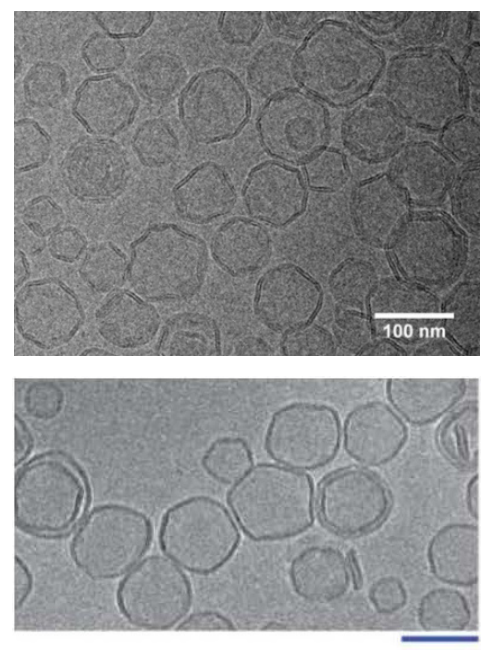

(b)

Figure 12. Aqueous dispersions of DPPC. (a) Top: Binary DPPC/water phase diagram, as published by Koynova and Caffrey (1998) [101]. Bottom: Schematic drawings of the fluid, liquid-disordered lamellar phase $\left(\mathrm{L}_{\alpha}\right)$ [84], and a snapshot from a molecular dynamics simulation of a DPPC bilayer in the $\mathrm{L}_{\alpha}$ state [106], of the the solid-ordered ripple phase $\left(\mathrm{P}_{\beta^{\prime}}\right)[107,108]$, and of the solid-ordered lamellar phase $\left(\mathrm{L}_{\beta^{\prime}}\right)$ [84]. (b) Cryo-TEM images of aqueous DPPC vesicle dispersions. From top to bottom: First and second: dispersion of DPPC in $50 \mathrm{mM} \mathrm{Mes} \mathrm{buffer}(\mathrm{pH}=6.0,150 \mathrm{mM} \mathrm{NaCl}, 5 \mathrm{mM}$ EDTA), as obtained by repeated freezing in liquid nitrogen and thawing at $T>T_{\mathrm{m}} \sim 41{ }^{\circ} \mathrm{C}$ (bar: $200 \mathrm{~nm}$ ), either quenched from $T=50{ }^{\circ} \mathrm{C}$ (first) or $T=25^{\circ} \mathrm{C}$ (second); see El Jastimi et al. (1999) [109]. Third: dispersion of DPPC in $10 \mathrm{mM}$ Tris- $\mathrm{HCl}$ buffer $(p H=7.4)$, as obtained by repeated extrusion through $100 \mathrm{~nm}$ polycarbonate membranes at $T=60^{\circ} \mathrm{C}$ (bar: $100 \mathrm{~nm}$ ), and quenched from $T=25^{\circ} \mathrm{C}$; see Farkuh et al. (2019) [110]. Fourth: dispersion of DPPC in $\mathrm{D}_{2} \mathrm{O}$, as obtained by repeated extrusion through $100 \mathrm{~nm}$ polycarbonate membranes (bar: $100 \mathrm{~nm}$ ), and quenched from $T=25^{\circ} \mathrm{C}$, see Matviykiv et al. (2019) [111]. Reproduced from [84], copyright (C Taylor and Francis, 2013; and with permission from [101], Elsevier, 1998; from [106], AIP Publishing, 1996; from [107], Springer Nature, 2000; from [109], Elsevier, 1999; from [110], Elsevier, 2019; and from [111], American Chemical Society, 2019.

\subsection{DOPA/Water Mixtures}

A tentative binary DOPA/water phase diagram was published by Lindlom et al. (1991) [113]; see Figure 13. DOPA is an anionic lipid. The $p H$ of the aqueous solution in which the lipid is dispersed-and, more importantly, the acidity at the aggregate surfacedetermines whether the head group of DOPA (or any other PA) is neutral, mono-anionic, or di-anionic [114]. Therefore, the extent of hydration and the aggregation behavior in 
the aqueous solution is expected to depend on $p H$ as well as on the counter ion type and composition of the aqueous solution $[115,116]$. The experimentally determined (apparent) $\mathrm{p} K_{\mathrm{a}}$ values for the di-protonated and mono-protonated forms of DOPA are $3.9 \pm 0.1$ and $8.6 \pm 0.3$, respectively [117]. The intrinsic $\mathrm{p} K_{\mathrm{a}}$ values may be lower [114]. For the binary phase diagram shown in Figure 13, the results obtained from the analysis of dispersions of the mono-sodium salt of DOPA in water are shown. As the phase diagram should represent the thermodynamic equilibrium situation, details provided for the sample preparation are worth mentioning here as an example. According to the description by Lindblom et al. (1991) [113], the samples were mixed in sealed tubes by centrifugation and several freezethaw cycles to achieve an equilibrated state "at rest". The samples were repeatedly analyzed by ${ }^{31} \mathrm{P}$ NMR measurements during a period of $2-3$ months. Such NMR measurements involve a spinning of the NMR tubes containing the samples and therefore may result in phase mixing if different phases would coexist at equilibrium. This needs to be taken into account when interpreting the NMR measurements. Moreover, measurements of aqueous DOPA dispersions at high temperature were found to be ambiguous since long term storage above $T \sim 50{ }^{\circ} \mathrm{C}$ resulted in DOPA degradation [113] most likely due to ester bond hydrolysis.

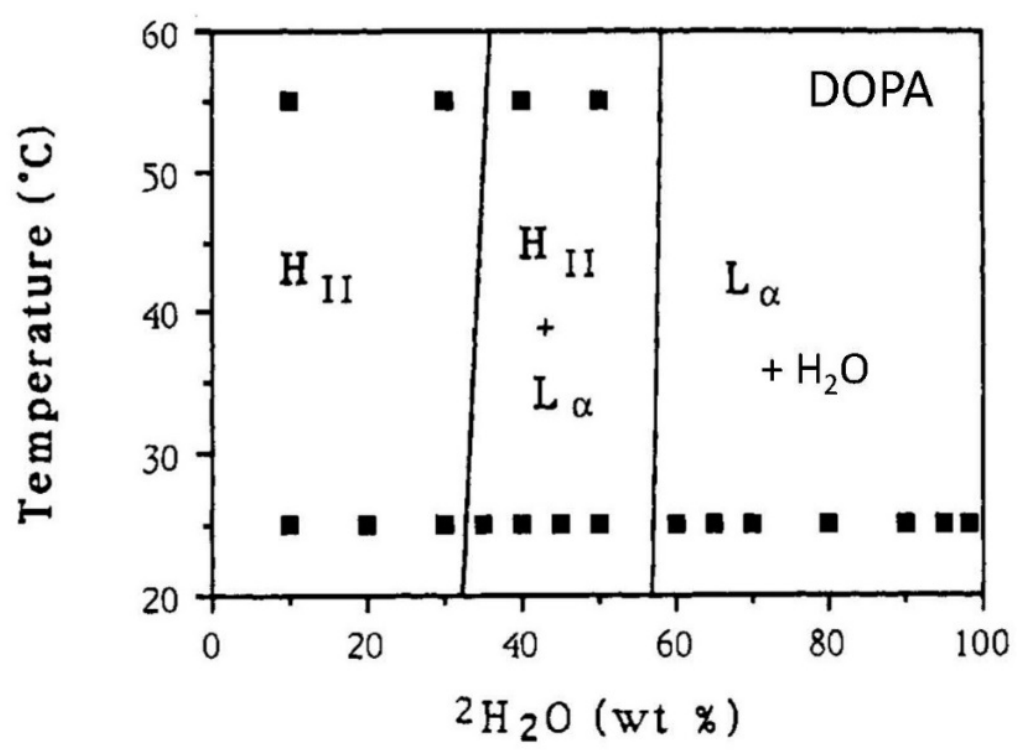

Figure 13. Aqueous dispersions of the mono-anionic form of DOPA. Tentative binary DOPA/water phase diagram, according to Lindblom et al. (1991) [113]. In the diagram shown, $\mathrm{H}_{2} \mathrm{O}$ was added in the high water content region as second phase $\left(\mathrm{L}_{\alpha}+\mathrm{H}_{2} \mathrm{O}\right) ; \mathrm{H}_{2} \mathrm{O}$ was omitted in the originally published diagram. Reproduced with permission from [113], American Chemical Society, 1991.

A shown in Figure 13, the mono-anionic form of DOPA forms a dispersed liquidcrystalline lamellar phase at high water content. This means that for these conditions, $p \approx 1$. As a consequence, dispersions of DOPA vesicles can be obtained under these conditions, which was confirmed experimentally [118]. Like DOPC and other long-chain phosphatidylcholines, DOPA is a bilayer-forming amphiphile. At low water content, however, an inverse hexagonal phase exists $\left(\mathrm{H}_{\mathrm{II}}, p>1\right)$.

\subsection{DOPE/Water Mixtures}

Kozlov et al. (1994) [119] published a calculated phase diagram for DOPE/water mixtures. A mirror image of this diagram is shown in Figure 14 (top). Phosphatidylethanolamines like DOPE are zwitterionic at $p H \sim 2.5-8.0$; the apparent $\mathrm{p} K_{\mathrm{a}}$ value of the ammonium group is about 10 [114]. 

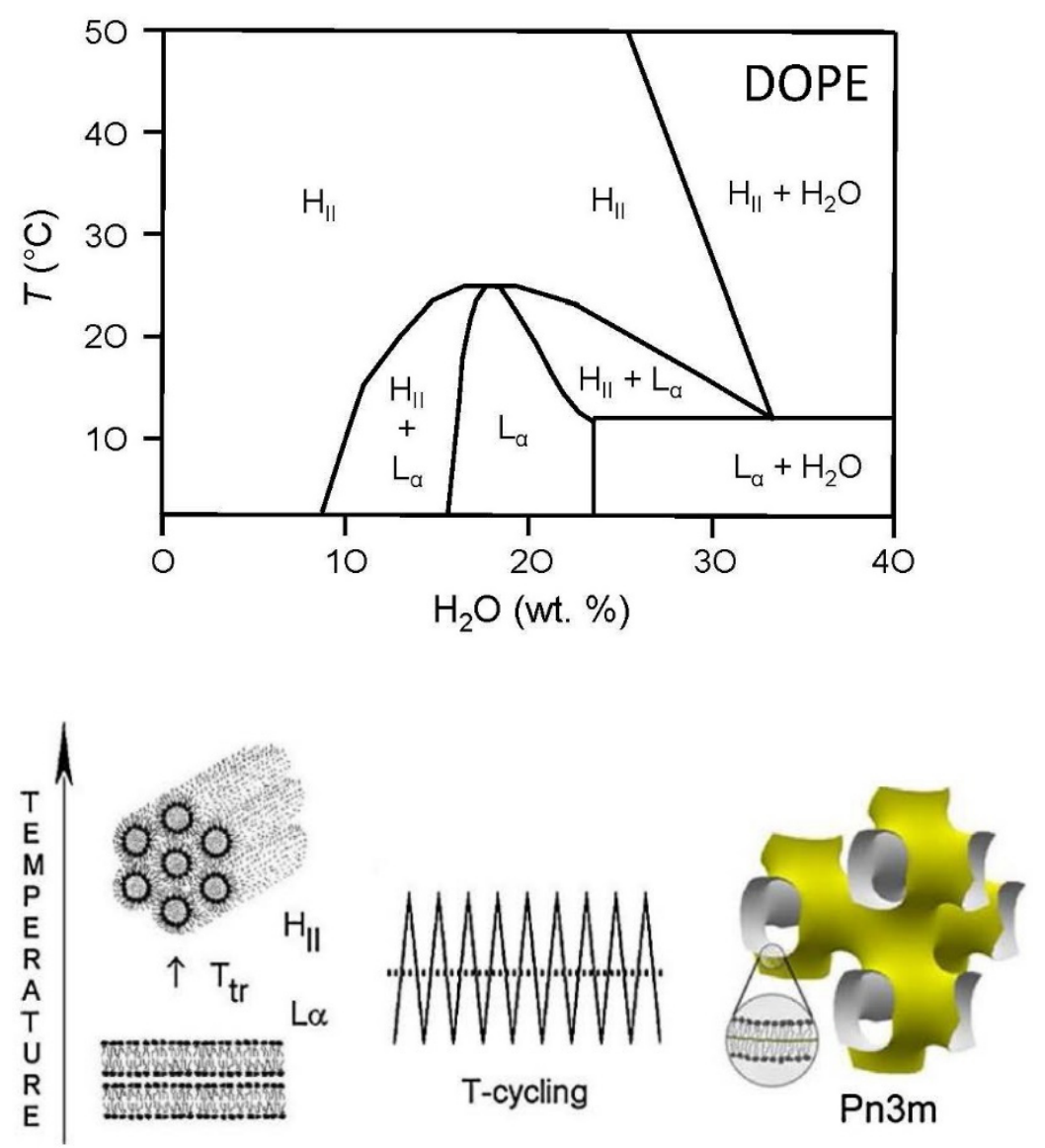

Figure 14. Aqueous dispersions of DOPE. Top: Calculated binary DOPE/water phase diagram, represented as mirror image of the diagram published by Kozlov et al. (1994) [119]. Bottom: Schematic representation of the (metastable) dispersed cubic phase formation upon temperature-cycling through a $\mathrm{L}_{\alpha}+\mathrm{H}_{2} \mathrm{O} \rightarrow \mathrm{H}_{\mathrm{II}}+\mathrm{H}_{2} \mathrm{O}$ transition, as published by Tenchov and Koynova (2017) [78]. Among the phosphatidylethanolamines investigated, the dispersed inverted cubic phase that formed from DOPE was Pn3m; in other cases, Im3m was obtained [78,120,121]. The experimental conditions in the case of DOPE were the following: $20 \mathrm{wt} \%$ DOPE in water, $1 \mathrm{M} \mathrm{NaSCN}$, after 50 temperature-cycles $\left(35-65{ }^{\circ} \mathrm{C}\right)$ and 7 days storage at $T=20^{\circ} \mathrm{C}$; the sealed sample was analyzed by X-ray diffraction measurements at $T=20^{\circ} \mathrm{C}$ [78]. Reproduced with permission from [119], Elsevier, 1994; and from [78], Elsevier, 2017.

Although there might be uncertainties concerning the phase boundaries, it is evident that at high water content and $T=25^{\circ} \mathrm{C}$, an inverted hexagonal phase $\left(\mathrm{H}_{\mathrm{II}}\right)$ coexists with water. This means that upon mechanically mixing the $\mathrm{H}_{\mathrm{II}}$ phase with water, a dispersion of hexosomes is obtained (not to be confused with "exosomes", i.e., extracellular vesicles of biological origin; see Section 6.5). Below $T \sim 10{ }^{\circ} \mathrm{C}$, a fluid lamellar phase $\mathrm{L}_{\alpha}$ coexists with water, i.e., an aqueous dispersion of vesicles is expected to form upon mixing the two phases at this low temperature. Lowering the water content for $\mathrm{T}>25^{\circ} \mathrm{C}$, a $\mathrm{H}_{\mathrm{II}}$ phase is expected to be present at thermodynamic equilibrium. Formation of the $\mathrm{H}_{\mathrm{II}}$ phase is understood on the basis of a small head group, which favors inverted structures (cone geometry of the amphiphile, $p>1$ ). The surprising behavior of DOPE is that a reduction in the water content at $15{ }^{\circ} \mathrm{C}<T<25{ }^{\circ} \mathrm{C}$ can result in a $\mathrm{H}_{\mathrm{II}} \rightarrow \mathrm{L}_{\alpha} \rightarrow \mathrm{H}_{\mathrm{II}}$ transformation [119]. Similarly, at high water content, a $\mathrm{L}_{\alpha}+\mathrm{H}_{2} \mathrm{O} \rightarrow \mathrm{H}_{\mathrm{II}}+\mathrm{H}_{2} \mathrm{O}$ transformation is evident from the phase diagram. This later transition is the basis for the possibility of forming a metastable (but "long-lived") dispersed inverted bicontinuous cubic phase from DOPE (Pn3m) at high water content, achievable by many temperature cycles [120-122]; see Figure 14 (bottom). This dispersed cubic phase formation is accelerated in the presence of certain dissolved compounds, e.g., the chaotropic solute sodium thiocyanate (NaSCN), which also increased the temperature of the $\mathrm{L}_{\alpha}+\mathrm{H}_{2} \mathrm{O} \rightarrow \mathrm{H}_{\mathrm{II}}+\mathrm{H}_{2} \mathrm{O}$ transition [58,78]. 


\subsection{A comparison of Lipid Vesicles and Micelles}

An isotropic aqueous solution of (spherical) micelles $\left(\mathrm{L}_{1}\right.$ or $\mathrm{M}_{\mathrm{I}}$ ) is considered by most researchers—but not by all $[62,123]$ —as a one phase system consisting of fluid micellar aggregates which are in rapid, dynamic equilibrium with non-associated, micelle-forming amphiphiles. The non-associated amphiphiles are also called "monomers" or "unimers", and the micelles often are considered as "pseudophase" [124]. An aqueous dispersion of fluid, i.e., liquid crystalline lipid vesicles consists of fluid vesicular aggregates that are in dynamic equilibrium with non-associated, bilayer-forming amphiphiles dissolved in the aqueous solution ( $\mathrm{L}_{\alpha}+$ aqueous solution, two phases). Figure 15 [125] illustrates the situations with a simplified representation of a snapshot across the two spherical aggregates, a vesicles (left) and micelles (right). Although smaller aggregates than an "optimal" micelle or an "optimally packed" vesicle may also exist in an equilibrated sample, the drawing highlights certain conceptual similarities between the two types of systems and also points to some differences with respect to (i) the concentration of non-associated amphiphiles and (ii) the time required to re-establish an equilibrated state once the equilibrium is disturbed (aggregate-unimer exchange kinetics). There are, however, also borderline cases, which clearly indicate that it is primarily the chemical structure of the amphiphile that dictates the properties, as outlined in the following.

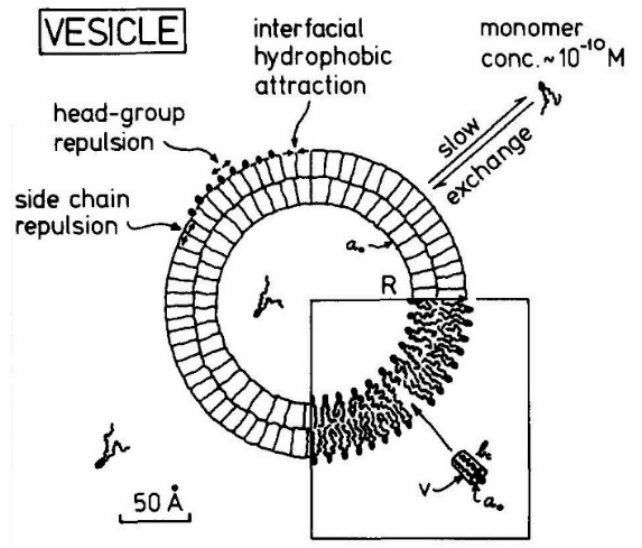

(a)

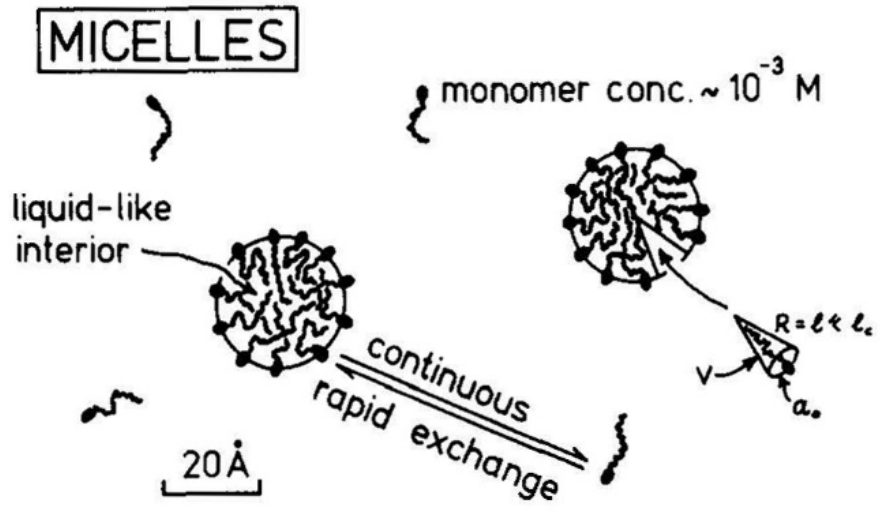

(b)

Figure 15. Schematic representation of the cross-section of (a) a spherical unilamellar vesicle and (b) spherical micelles, as published by Israelachvili et al. (1977) [125]. The drawing illustrates (i) the packing of the amphiphiles as geometric objects (see Equation (1) in Section 2.3), (ii) the interactions at the interface of the aggregates, and (iii) the exchange of the amphiphiles between the aggregated state and the molecularly dissolved state. Typical values for the concentration of non-associated amphiphiles ("monomers") are indicated; see also the text. Reproduced with permission from [125], Elsevier, 1977.

The concentration of non-associated amphiphiles in the case of typical micelle-forming amphiphiles is much higher than the concentration of non-associated amphiphiles in the case of bilayer-forming amphiphiles; the unimer concentration corresponds in a first approximation to the $C M C$ or $C V C$, respectively, usually with $C M C>>C V C$. However, depending on the chemical structure of the amphiphile, the $C V C$ values can also be relatively high [70]. One example is the $C V C$ for decanoic acid vesicles: about $20-40 \mathrm{mM}$ at $p H=7.1-7.3[65,70,126]$.

Diluting a micellar solution below the $C M C$ results in a complete disintegration of the micelles. In the case of a vesicle dispersion, the situation is the same: dilution below the $C V C$ results in a disintegration of the vesicles. Since the $C V C$ for conventional bilayer-forming phosphatidylcholines like POPC is, however, very low (CVC(DPPC) (4.6 \pm 0.5$) \times 10^{-10} \mathrm{M}$ [127], or even lower [128]), concerns about vesicle disintegration are only appropriate in extremely dilute samples. To illustrate this, let us assume that one $100 \mathrm{~nm}$-sized LUV of POPC is constituted by $9.2 \times 10^{4}$ POPC molecules (see legend of Figure 1 ). For a dilution of a LUV dispersion from $1 \mathrm{mM}$ POPC to $1 \mu \mathrm{M}$ POPC, for example, each vesicle on average 
would lose 10 POPC molecules, only. This loss of POPC molecules from the vesicle can be ignored. In the case of a decanoic acid vesicle dispersion prepared a $p H \sim 7.2$, however, vesicles do not exist below 20-40 mM decanoic acid + decanoate.

\subsection{Summary}

The phase diagrams shown and discussed in this Chapter are illustrations of how selected biological amphiphiles aggregate in aqueous solution under certain conditions in terms of amphiphile concentration, composition of the aqueous solution, and temperature for a standard pressure of about 1 bar. A large number of similar phase diagrams are also known for non-natural, fully synthetic amphiphiles that are important for industrial applications [129,130], for example, for aqueous mixtures of anionic sodium dodecylsulfate (SDS) [131], cationic hexadecyltrimethylammonium bromide (CTAB) [132], or non-ionic $n$-hexadecyl octaethylene glycol ether $\left(\mathrm{C}_{16} \mathrm{EO}_{8}\right)$ [133] and n-dodecyl octaethylene glycol ether $\left(\mathrm{C}_{12} \mathrm{EO}_{8}\right)$ [134], all four compounds being typical micelle-forming amphiphiles. Conceptually, there is no difference between the aggregation behavior of synthetic amphiphiles and the aggregation behavior of naturally occurring (biological) amphiphiles; the focus in this article, however, is on biological amphiphiles.

Binary amphiphile/water diagrams show for which conditions at thermodynamic equilibrium a single liquid crystalline phase forms-and how the amphiphiles are organized in the aggregated state comprising this phase-or whether two (or even more) phases coexist. The molecular arrangement of the amphiphiles in a phase represents the energetically most favorable situation due to the amphiphile's self-assembly, primarily on the basis of hydrophobic attractions, electrostatic repulsions, and maximal entropy [18]. The fluid lamellar phase $\left(\mathrm{L}_{\alpha}\right)$ formed from biological amphiphiles (lipids), the different cubic phases (e.g., the inverse bicontinuous cubic phase $\mathrm{Pn} 3 \mathrm{~m})$, or the two hexagonal phases $\left(\mathrm{H}_{\mathrm{I}}\right.$ and $\left.\mathrm{H}_{\mathrm{II}}\right)$ are of particular interest for many applications, due to the dispersibility of these phases in excess aqueous solution, where these phases coexist at thermodynamic equilibrium with an aqueous solution, to form in the dispersed state kinetically trapped lipid vesicles (liposomes) [21-23,135-138], cubosomes [80,139-144], or hexosomes [141,144,145]. If other amphiphiles or non-amphiphilic hydrophobic or hydrophilic molecules are present as well, the aggregation behavior can be very complex, with the possible formation of phases that are not present in the binary system [146]. Moreover, added molecules may (i) stabilize or (ii) completely destabilize the state of the dispersed phase, or (iii) they may alter the properties of the dispersed phase in a desired and controlled way.

Examples for (ii) and (iii) are micelle-forming amphiphiles (detergents). If added to lipid vesicles, detergents permeabilize lipid vesicles (liposomes) at low concentration and low detergent-to-lipid ratio by forming mixed lipid-detergent vesicles with altered properties as compared to detergent-free vesicles [147]. In the presence of high enough amounts of detergent, a dispersion of lipid vesicles will transform into a solution of mixed detergent-lipid micelles [147-152], a process known as membrane or liposome solubilization.

Concerning (i), in Chapter 3, the colloidal (physical) stability of lipid vesicles and cubosomes is discussed and how their stability can be increased for in vitro or in vivo applications.

In the subsequent Chapters 4 and 5, the focus will be on lipid vesicles, discussing concepts about the preparation methods (Chapter 4) and approaches for loading vesicles with water-soluble molecules and for the surface functionalization of vesicles (Chapter 5). Finally, the selected examples of the application of lipid vesicles (and lipid nanoparticles) in Chapter 6 should highlight some of the innovative ideas that were developed over the years about this type of lipid aggregates, emerging from properties that were previously determined by a large number of fundamental studies. 


\section{Increasing the Stability of Aqueous Dispersions of Lipid Vesicles and Cubosomes}

The physical stability of lipid vesicle dispersions very much depends on the type and concentration of bilayer-forming amphiphile (or type of lipid mixture) used, on the way the dispersion is prepared (see Chapter 4), the temperature, and the composition of the aqueous solution. Often, aqueous lipid vesicle dispersions are colloidally rather stable for several weeks or months if analyzed by dynamic light scattering [153-158]. As an example, fluid, zwitterionic DOPC or POPC vesicle dispersions that were prepared in PBS $(p H=7.4$, phosphate buffered saline composed of $137 \mathrm{mM} \mathrm{NaCl}, 2.7 \mathrm{mM} \mathrm{KCl}$, $10 \mathrm{mM} \mathrm{Na}_{2} \mathrm{HPO}_{4}, 2 \mathrm{mM} \mathrm{KH}_{2} \mathrm{PO}_{4}$ ) by polycarbonate membrane extrusion by using for final extrusions membranes with average pore diameters of $100 \mathrm{~nm}$ were stable at $T=25$ or $42{ }^{\circ} \mathrm{C}$ for at least one week [155] (measurements beyond this time were not carried out). Another example is the high colloidal stability of vesicles that were prepared from mixtures of partially hydrogenated egg PC and egg phosphatidylglycerol (egg PG) in $50 \mathrm{mM}$ HEPES buffer, $p H=7.0$, using again polycarbonate membrane extrusion (with $200 \mathrm{~nm}$-membranes for final extrusions), and then stored for 40 days at $T=40{ }^{\circ} \mathrm{C}$ [153]. The physical stability of phosphatidylcholine vesicle dispersions originates in part at least from repulsive "hydration forces" $[18,159,160]$ due to the presence of water molecules that hydrate the polar head groups on the surface of the vesicles (Figure 16). These hydration forces act between two vesicles that come in close contact and thereby prevent vesicle aggregation, which would be the result of attractive van der Waals (hydrophobic) forces. If required, the colloidal stability of zwitterionic lipid vesicle dispersions can be increased by adding charged amphiphiles (e.g., anionic phosphatidylglycerol (PG) $[153,161,162])$ at amounts that do not prevent bilayer formation and that do not alter the vesicle membrane properties in an undesired way [163-167]. The addition of charged amphiphiles results in a stabilization of the vesicle dispersion due to inter-vesicular electrostatic repulsions [168], which prevent vesicle aggregation and fusion to form multilamellar vesicles as lamellar phase $\left(\mathrm{L}_{\alpha}\right)$ that would separate from the aqueous solution in which the vesicles originally were dispersed.

Another possibility for increasing the colloidal stability of lipid vesicles is to use a small fraction of a synthetic amphiphile that has a bulky polar head group, usually polyethylene glycol (PEG). Two of the PEGylated amphiphiles are 1,2-distearoyl-sn-glycero3-phosphoethanolamine-PEG750 (DSPE-PEG750, with about 17 ethyleneoxide moieties $\left(-\mathrm{CH}_{2} \mathrm{CH}_{2} \mathrm{O}-\right)$ in the head group, with a terminal methoxy group $\left(-\mathrm{OCH}_{3}\right)$, corresponding to an average molar mass of $M \sim 750 \mathrm{~g} / \mathrm{mol}$ [169] or Tween 80 (also known as polysorbate 80, polyoxyethylene (20) sorbitan monooleate) [170]. Vesicles containing such amphiphiles at the optimal amount do not aggregate easily due to steric hindrance by the hydrated polymer chains, as long as the polymer chain density on the vesicle surface is high enough (Figure 16).

Lipid vesicles containing PEGylated lipids, for example DSPE-PEG2000 (about 45 ethyleneoxide units, with a terminal methoxy group, $M \sim 2000 \mathrm{~g} / \mathrm{mol}$ ), at optimal PEG chain length and surface density (Figure 16) are used in intravenously administered drug delivery systems (see Section 6.2). Such sterically stabilized vesicles ("stealth liposomes" $[25,171-175]$ ) do not interact so easily with opsonins (i.e., serum proteins of the immune system). Therefore, vesicles injected into the blood circulation are not removed immediately by phagocytes of the immune system, i.e., monocytes in the blood and macrophages in tissue, and therefore stay longer in the blood circulation than vesicles that do not contain such water soluble polymers on their surface [25]. With an extended period of time in the blood circulation, stealth liposomes eventually reach the target, for example tumor cells, more efficiently than non-PEGylated liposomes of otherwise similar size and composition [172]. Compared to the increased colloidal stability of PEGylated vesicles in vitro, where vesicle-vesicle interactions are minimized, the role of the PEG chains on the surface of the stealth liposomes play in vivo is to minimize vesicle-protein (opsonin) interactions. 
Interaction between zwitterionic phosphatidylcholine bilayer surfaces

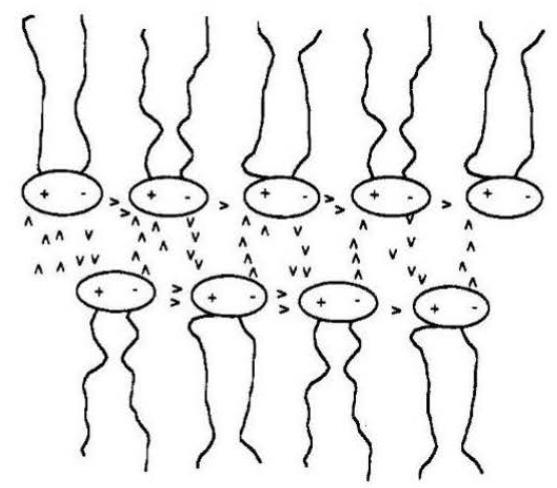

(a)

\section{Sterically stabilized liposome}
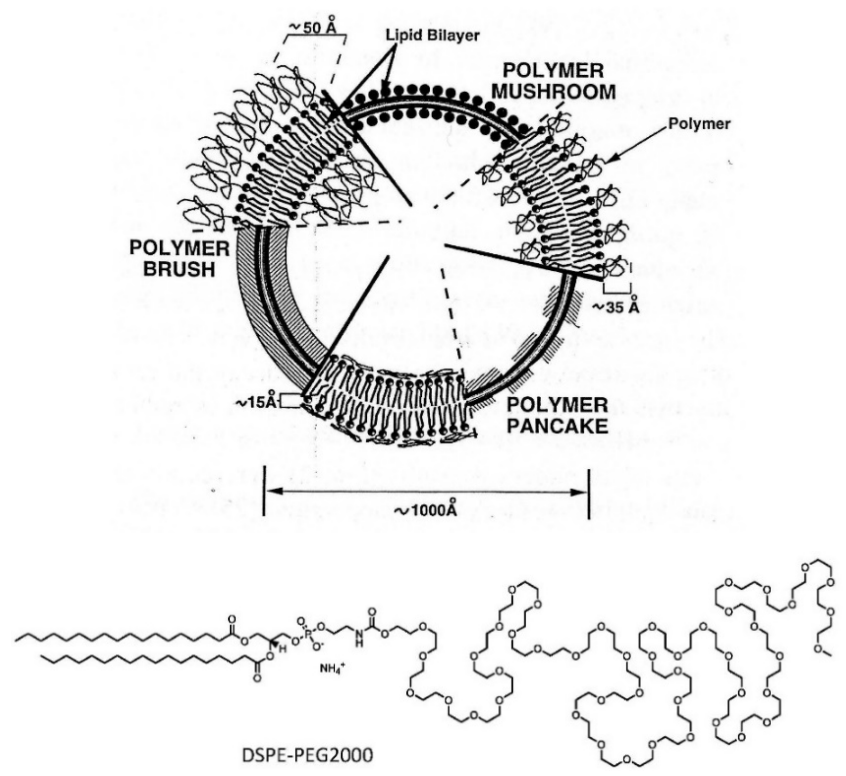

(b)

Figure 16. Possible way of improving the physical stability of lipid vesicles in vitro and in vivo by using PEGylated lipids. (a) Schematic illustration of the localization of water molecules on the polar surface of fluid zwitterionic PC bilayers, as sketched by Rand et al. (1988) [159], resulting in bilayer repulsions due to "hydration forces". Each phosphoryl head group is capable of polarizing water molecules in opposite directions. For an inter-bilayer contact, a force has to be applied to remove the water molecules from the inter-bilayer space. This hydration force prevents bilayer aggregation in the $\mathrm{L}_{\alpha}$ phase and prevents fluid lipid vesicle aggregation in lipid vesicle dispersions at $T>T_{\mathrm{m}}$. Other repulsive interactions between dispersed lipid vesicles are electrostatic interactions in the case of charged vesicles and steric repulsions for vesicles containing water-soluble polymers attached to some of the polar head groups on the surface of the vesicles ("PEGylated liposomes") [172,173]. (b) Top: Schematic representation of the situation on the surface of PEGylated liposomes, depicting various possible PEG chain densities (pancake, mushroom, or brush regime), from Čeh et al. (1997) [173]. For in vivo applications of "stealth liposomes" [172,173], the stealth effect is achieved for PEGylated liposomes in the brush regime [175]. Bottom: Chemical structure of DSPE-PEG2000 with 45 ethyleneoxide repeating units and a terminal methoxy group. Reproduced with permission from [159], American Chemical Society, 1988; and from [173], Elsevier, 1997.

Amphiphiles with PEG as polar head group often are also used to increase the colloidal stability of cubosome (or hexosomes) dispersions [80,176]. Examples are DSPE-PEG750 [80], PEGylated monoolein (MO-PEG2000), or non-ionic triblock copolymers Pluronic ${ }^{\circledR}$ F127 (also known as poloxamer 407, $\mathrm{EO}_{100} \mathrm{PO}_{65} \mathrm{EO}_{100}$ [177]), or Pluronic ${ }^{\circledR} \mathrm{F} 68$ (also known as poloxamer 188, $\mathrm{EO}_{76} \mathrm{PO}_{29} \mathrm{EO}_{76}$ [177]) $[80,140,145], \mathrm{EO}$ and $\mathrm{PO}$ being ethylenoxide and propylenoxide repeating units, respectively. In these cases, the PEGylated amphiphiles should form a protecting coat on the surface of the cubosome particles. With this, cubosomecubosome interactions should be minimized, and the formation of a separate cubic phase should be avoided. If the stabilizing amphiphiles diffuse into the internal cubic phase and mix with the cubosome-forming amphiphiles, the original cubic phase might be altered, and partial vesicle formation might occur [80].

\section{Lipid Vesicle Dispersions Obtained by Guided Assembly}

\subsection{Overview of the Concepts for the Formation of Large or Giant Unilamellar Vesicles}

As mentioned in Chapter 2, aqueous lipid vesicle dispersions usually-but not always [178-180]—are only kinetically stable. They are obtained by "guided assembly" procedures. With these procedures, bilayer-forming amphiphilic lipids are forced to arrange as curved self-closed bilayers (vesicles) of desired average curvature (i.e., desired average vesicle size). In the majority of cases, the conditions are chosen such that the vesi- 
cles have a spherical shape, although the formation of (transiently) non-spherical vesicles is also possible [181-185].

There are various established methods for the formation of spherical unilamellar vesicles with average sizes of either about $100 \mathrm{~nm}$ (known as "large unilamellar vesicles", LUVs) or several micrometers (so-called "giant unilamellar vesicles", GUVs). These methods are summarized in many review articles or in books [161,186-195]. In Figure 17, some of the key concepts for the formation of LUVs and GUVs are summarized.

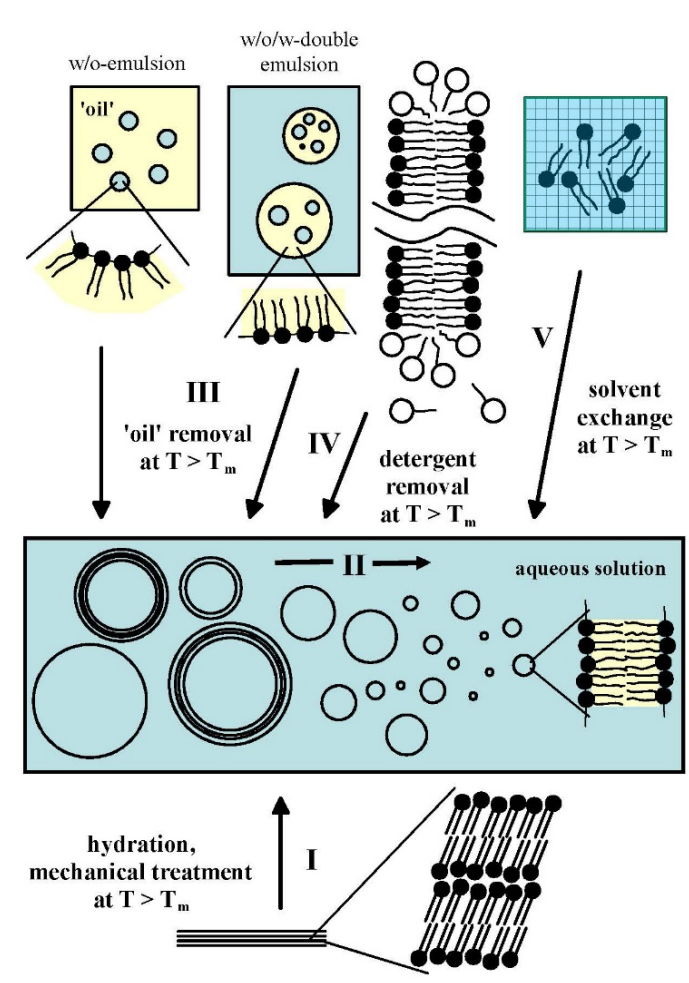

(a)

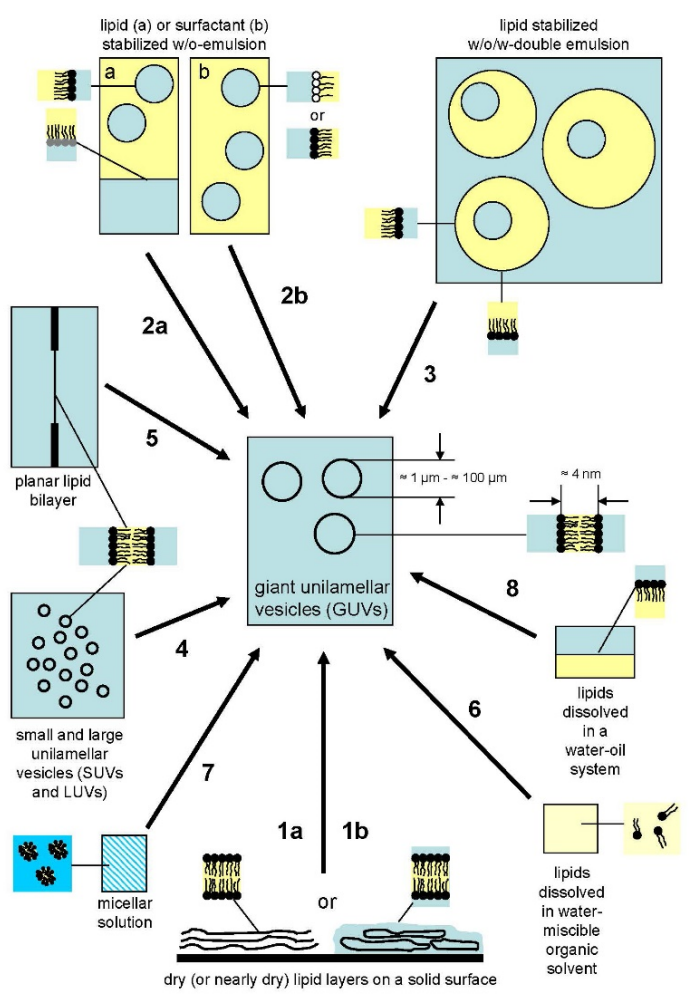

(b)

Figure 17. Schematic representation of the concepts of some of the methods that were developed for the formation of (a) large unilamellar vesicles (LUVs), with diameters in the range of $100 \mathrm{~nm}$, and (b) giant unilamellar vesicles (GUVs), with diameters above $1 \mu \mathrm{m}$. (a) Multilamellar vesicles (MLVs) are usually obtained by dispersing a dry film of bilayer-forming lipids at $T>T_{\mathrm{m}}$ in an aqueous solution (I). The obtained MLV dispersion can be sized down to LUVs by polycarbonate membrane extrusion at $T>T_{\mathrm{m}}$. Sonication of MLVs yields sonicated (or small) unilamellar vesicles (SUVs). The reduction in vesicle size and lamellarity is represented by "II" as process from left to right in the horizontal box. Sub-micrometer-sized unilamellar vesicles can also be obtained from $w / o$-emulsions or $w / o / w$-double emulsions containing bilayer-forming lipids (III, well-known is the "reverse-phase evaporation method"); from an aqueous solution of mixed detergent-lipid micelles (IV, "detergent removal method" or "detergent depletion method") or from an ethanolic solution containing bilayer-forming lipids (V, known as "ethanol injection method"). (b) GUVs can be obtained by a careful hydration of a thin film of bilayer-forming lipids deposited on a solid surface (1a and 1b, "spontaneous hydration method" in its simplest version). The hydration may be done on a conducting surface in the presence of an electric field ("electroformation method"). With these methods (1a,1b), the GUVs usually remain attached to the surface at the place where the lipids originally were deposited. Other methods for GUV formation are based on the initial formation of micrometer-sized water droplets in a water-immiscible oil, followed by either the transfer of the droplets from the oil into an aqueous solution ("droplet transfer method", 2a), or by first freezing the droplets, followed by coating with lipids in the frozen state and hydration during droplet melting ("lipid-coated ice droplet hydration method", 2b). In alternative procedures, the initial states of the lipids are either in the form of $w / o / w$-double emulsions (3), dispersions of SUVs or LUVs (4), planar lipid bilayers (5), lipids dissolved in a water-miscible organic solvent (dioxan or tetrahydrofuran, for example, 6), micelles (7), or a water-oil system (8). For details of the different procedures, see text and the original literature cited in the two references where the schemes were published first [191,192]. Reproduced with small modifications from [191], American Scientific Publishers, 2004, and with permission from [192], WILEY-VCH Verlag GmbH \& Co. KGaA, 2010. 
For the formation of LUVs (Figure 17a), one well-known method is the "extrusion technique" [112,196-200]. A heterogeneous dispersion of multilamellar vesicles (MLVs) is forced to pass through the cylindrical pores of polycarbonate membranes at $T>T_{\mathrm{m}}$, i.e., under conditions at which the vesicle bilayers are in the liquid-disordered $\left(l_{\mathrm{d}}\right)$, fluid state. During this passage through the pores, MLVs get deformed and most likely undergo a pearling process during which the vesicle membranes are ruptured and then finally re-close at the outlet of the pores [201-203], to an average size which correlates with the size of the pores. This procedure is done repeatedly by using membranes with 400,200 , and finally $100 \mathrm{~nm}$ pore diameters $[197,204]$. The smaller the pore size is, the more homogenous the obtained LUVs are, although the presence of oligolamellar vesicles in the final dispersions can not be excluded, depending of the lipid type and the aqueous solution [205]. Often, final extrusions are made with membranes consisting of $100 \mathrm{~nm}$ pores, yielding LUVET $_{100}$, an abbreviation that is used to indicate that the vesicles obtained were LUVs prepared by the extrusion technique (ET), using for final extrusions the pore diameter indicated in the subscript (in $\mathrm{nm}$ ). The diameter of the vesicles obtained with $100 \mathrm{~nm}$ pore membranes usually is about $100 \mathrm{~nm}$. Using larger pores, often a substantial amount of oligolamellar vesicles (OLVs) are also present in the final vesicle dispersion, and the vesicle size is not so homogenous, with an average diameter that is smaller than the pore diameter [197]. Conceptually, polycarbonate membrane extrusion is a mechanical treatment of a vesicle dispersion for the reduction of average size and lamellarity (indicated in Figure 17a in the horizontal box with "II" as size and lamellarity reduction process occurring from left to right). Sonication at $T>T_{\mathrm{m}}$ can also be used as alternative method to decrease vesicle size and lamellarity, yielding SUVs (sonicated or small unilamellar vesicles) $[206,207]$. The initial dispersion of MLVs often is obtained by hydrating at $T>T_{\mathrm{m}}$ a dry film of bilayerforming lipids deposited on a solid surface [95]; see Figure 17a (I). Depending on the lipid film thickness, on the type of lipid, and on how the hydration is made, GUVs may also form; see Figure 17b (1a).

Aqueous vesicle dispersions can also be obtained by starting with a water-in-oil emulsion (w/o-emulsion). After removal of the oil (a water-immiscible organic solvent) and addition of excess aqueous solution, a dispersion of vesicles is obtained. One of the original procedures is known as "reverse phase evaporation method" [208]; see Figure 17a (III). Like for any of the other methods mentioned here, a successful application in terms of desired quality of the vesicle dispersion obtained depends on the experimental details, such as chemical structure of the lipids used, lipid concentration, composition of the aqueous solution, etc. Starting with a water-in oil-in water $(w / o / w)$-double emulsion, aqueous dispersions of vesicles can be obtained as well after the oil is removed.

Another approach is to start with an aqueous solution of mixed micelles consisting of a bilayer-forming lipid and a micelle-forming amphiphile (a detergent) at conditions, where mixed detergent-lipid micelles form; see Figure 17a (IV). The characteristics of this mixed micelle solution is that the concentration of non-associated detergent molecules is much higher than the concentration of non-associated lipid molecules, i.e., $C M C$ (detergent) $>>C V C$ (lipid); see Section 2.15. Non-associated detergent molecules are continuously removed from the system, for example, by dialysis or by size exclusion chromatography. A new equilibrium will be established due to the exchange of the detergent molecules between the mixed micelles and the aqueous solution, until mixed lipid-detergent vesicles form and finally vesicles that are almost free of detergent. The entire process is known as "detergent depletion method" [209-211].

The "ethanol injection method" is based on the addition of an ethanol solution, in which bilayer-forming, ethanol-soluble amphiphiles are dissolved, to an aqueous solution, in which vesicle formation is desired to occur; see Figure 17a $(\mathrm{V})$. Ethanol is miscible with water. As a consequence, the ethanol molecules solvating the hydrophilic and hydrophobic parts of the lipids in the ethanol solution will partition into the aqueous solution when the ethanol solution and the aqueous solution are mixed. With this, the lipids self-organize 
to form bilayers that self-close to vesicles, the size of the vesicles being dependent on the experimental conditions [212-218].

For the formation of GUVs (Figure 17b), the most successful procedures are, and must be, different from the ones used for the formation of LUVs (Figure 17a). There are two different kinds of GUV preparations that one can obtain by the different procedures: First, GUVs that form on a solid surface and usually are left attached to this surface for investigations and then investigated by light or confocal fluorescence microscopy (in the latter case by using hydrophobic, amphiphilic, or hydrophilic fluorescent probe molecules); second, GUVs that are part of an aqueous dispersion, which is analyzed or applied as dispersion, just like in the case of a dispersion of LUVs.

Surface-attached GUVs can be obtained by a careful hydration of a thin film of completely dried (or optimally wetted) bilayer-forming lipids deposited on a solid surface; see Figure $17 \mathrm{~b}(1 \mathrm{a}, \mathrm{b})$ [219]. Without any additional guiding of the lipid hydration process, this method is called "spontaneous swelling" or "gentle hydration method" [220]. Using as solid surface a conductive glass or an electrode (platinum wire), the swelling and hydration of the lipids can be promoted ("guided") in a controlled way by applying an electric field. This procedure originally was developed by Angelova and Dimitrov (1986) [221] and is known as "electroformation method" [222] and is widely used [222-227].

For obtaining GUV dispersions, there is one method which is often applied. It is called "droplet transfer method" because the method is based on the transfer of micrometersized aqueous droplets present in a $w / o$-emulsion into an aqueous solution; see Figure $17 \mathrm{~b}$ (2a) [228]. During this process, monolayer-stabilized aqueous droplets of the w/o-emulsion are converted into bilayer-stabilized aqueous droplets (present as GUVs in the aqueous medium into which the droplets are transferred). The required second (outer) monolayer, which is needed for coating the droplets, is acquired during the migration of the droplets from the lighter $w / o$-emulsion across a monolayer-stabilized interface into the lower, denser aqueous solution. Although this procedure is understood conceptually, the experimental details are very critical for obtaining in a reproducible way the desired GUV dispersion [194,229-231]. Moreover, the possible presence of oil in the GUVs needs to be considered. Related to this method is the "lipid-coated ice droplet hydration method" [232], where monodisperse aqueous droplets in an organic solvent (hexane) are first formed by microchannel emulsification, followed by freezing of the droplets in liquid nitrogen, removal of the organic solvent, and finally hydration with an aqueous dispersion of LUVs, succeeded by droplet melting through a raise in temperature; see Figure $17 \mathrm{~b}(2 \mathrm{~b})$. With another method that requires the use of oil, an initial $\mathrm{w} / \mathrm{o} / \mathrm{w}$-double emulsion is prepared from which the oil is removed; see Figure $17 \mathrm{~b}$ (3). Depending on the oil present, significant rearrangements of the amphiphiles have to take place, which makes it difficult to understand how the final vesicles should become unilamellar.

The other methods for GUV formation that are shown in Figure $17 \mathrm{~b}$ are based on either the fusion of a very large number of SUVs or LUVs (4), or, for example, on the jet-blowing of a small aqueous volume onto a planar lipid bilayer [233-235] (5). Although for bilayer-forming biological lipids it may not work very well, the mixing of a solution of amphiphiles dissolved in a water-miscible solvent with an aqueous solution (6, related to the "ethanol injection method" for the formation of LUVs) is another way for potentially obtaining GUVs. Other methods mentioned in Figure $17 \mathrm{~b}$ are based on either the initial use of an aqueous solution of (mixed) micelles (7), or amphiphiles dissolved in a water-oil system (8).

\subsection{Reproducible Large Scale Formation of LUV Dispersions with the "Ethanol Injecton Method"}

Over the last years, the general concept of the "ethanol injection method" was developed further for large scale applications by optimizing the engineering part of this method so that even the reproducible production of a commercial liposomal antifungal product became possible (Pevaryl Lipogel) [236,237]. Based on recent developments in the use of microfluidic systems for the controlled mixing of an ethanolic solution containing bilayer- 
forming lipids and an aqueous solution [238-241], Kuwamura et al. (2020) [218] published a systematic investigation for the continuous production of lipid vesicles by using a simple procedure involving an inexpensive V-shaped solvent mixer unit; see Figure 18. The liposomes obtained had diameters in the range of $50-70 \mathrm{~nm}$ (with narrow size distributions), depending on the experimental parameters used. Different lipid mixtures were used, including DSPC:cholesterol:DSPE-PEG2000 (10:10:1, molar ratio), at 6.82 M DSPC in ethanol and a physiological saline solution ( $154 \mathrm{mM} \mathrm{NaCl}$ in water) as aqueous solution [218]. The liposomes obtained were stable when stored at $T=4{ }^{\circ} \mathrm{C}$ for up to 28 days without significant change in size. Encapsulation experiments by using as aqueous solution $125 \mathrm{mM}$ closo-dodecaborate in water and a flow rate of $12 \mathrm{~mL} / \mathrm{min}$ indicated a rather high entrapment efficiency for this water soluble compound with entrapment yields varying with the flow rates used [218]. Closo-dodecaborate is a water-soluble icosahedral boron cluster consisting in its sodium form of 12 boron atoms and two sodium counter ions, $\mathrm{Na}_{2} \mathrm{~B}_{12} \mathrm{H}_{12}$. This compound was used as model compound for sodium borocaptate $\left(\mathrm{Na}_{2} \mathrm{~B}_{12} \mathrm{H}_{11} \mathrm{SH}\right)$, which is used in clinical treatments of tumors ("boron neutron capture therapy") [242]. After separation by ultracentrifugation of the non-entrapped clusters from the vesicles containing entrapped $\mathrm{Na}_{2} \mathrm{~B}_{12} \mathrm{H}_{12}$, followed by resuspension of the pellets obtained in the physiological saline solution, the experimentally determined entrapment varied between 1.6 and $2.8 \mathrm{~mol}$ boron atom of the cluster to phosphorus atom of the lipid [218].
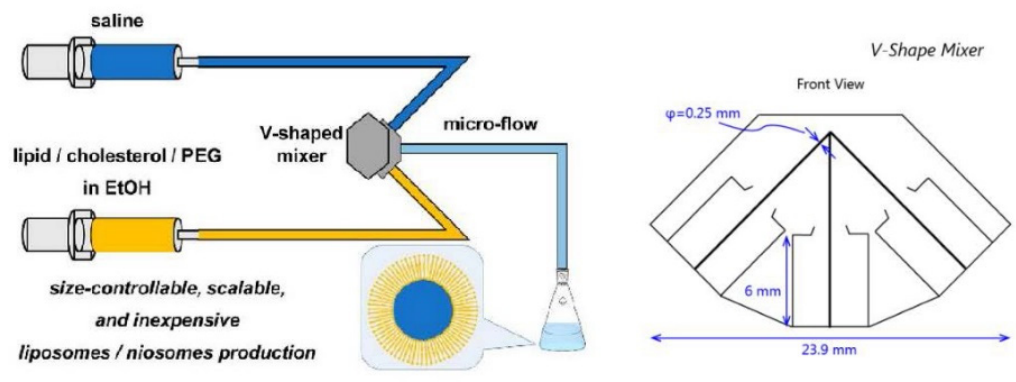

(a)

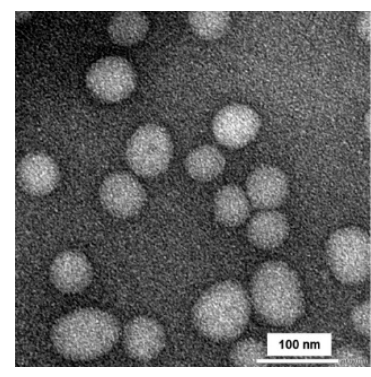

(b)

Figure 18. Size-controlled and scalable production of LUVs based on the "ethanol injection method" by using a V-shaped mixer (diameter: $250 \mu \mathrm{m}$ ) and a micro-flow reactor, with an outlet tube diameter of $1 \mathrm{~mm}$, as published by Kawamura et al. (2020) [218]. (a) Schematic representation of the set-up and details of the stainless steel V-mixer. The procedure was shown to be applicable for the preparation of lipid vesicles from mixtures of amphiphilic phospholipids (e.g., DSPC:cholesterol:DSPEPEG2000 (10:10:1, molar ratio) as well as for vesicles prepared from non-ionic surfactants ("niosomes"). The size of the obtained vesicles was in the range of 50-70 nm depending on the experimental conditions; see text and [218]. (b) Negative staining transmission electron microscopy image of one of the lipid vesicle dispersion obtained, analyzed after mixing with an EM stain solution (containing a lanthanide salt) and then analyzed in the dry state at room temperature (bar: $100 \mathrm{~nm}$ ) [218]. Reproduced with permission from [218], American Chemical Society, 2020.

In another investigation, a microfluidic glass capillary version of the "ethanol injection method" was used successfully for the preparation of lipid vesicles containing entrapped enzyme molecules (bovine erythrocytes $\mathrm{Cu}, \mathrm{Zn}$-superoxide dismutase, SOD); see Costa et al. (2021) [243] and Figure 19. Using an ethanolic solution of egg PC:cholesterol:DSPEPEG2000 (1.85:1:0.15, molar ratio), at a total lipid concentration of $48 \mathrm{mM}$, and an aqueous solution consisting of $145 \mathrm{mM} \mathrm{NaCl}, 10 \mathrm{mM}$ citric acid, $p H=6.0$, and $75 \mu \mathrm{g} / \mathrm{mL}$ SOD, a dispersion of LUVs was obtained at a flow rate of $25 \mathrm{~mL} / \mathrm{h}$. The vesicles had a diameter of $135 \pm 41 \mathrm{~nm}$ [243]. Separation of the vesicles containing entrapped SOD from non-entrapped SOD was carried out by ultracentrifugation followed by re-suspending of the pellets obtained in the $p H=6.0$ buffer solution. The entrapment yield obtained was high, and the entrapped enzyme molecules were shown to be catalytically active, even in an intravenous in vivo application of the SOD containing vesicles as anti-inflammatory nanosystem [243]. From the reported protein to phospholipid ratio of the SOD-containing 
vesicles prepared, about $0.9 \mu \mathrm{g} / \mu \mathrm{mol}$ [243], the approximate average amount of enzyme molecules per lipid vesicle can be estimated. It is about 3-4 SOD molecules per vesicle. This estimation is based on several assumptions, but nevertheless, it is a useful exercise since it provides a rough view of the molecular situation (see also the legend of Figure 1). The calculations were made as follows. We assumed that all vesicles obtained by the microfluidic method used are unilamellar and uniform in size with an outer vesicle diameter of $135 \mathrm{~nm}$ and a vesicle bilayer thickness of $4 \mathrm{~nm}$. With this, the outer and the inner vesicle surface areas can be calculated and with this the total interfacial area of one vesicle membrane $\left(\sim 1.08 \times 10^{5} \mathrm{~nm}^{2}\right)$. Assuming that the protein content determined in the vesicle dispersion after removing non-entrapped SOD molecules $(0.9 \mu \mathrm{g}$ per $\mu \mathrm{mol}$ phospholipid) reflects the SOD content, with $M(\mathrm{SOD})=32,500 \mathrm{~g} / \mathrm{mol}$ [244] $\left(\sim 2.83 \times 10^{-11}\right.$ mol SOD per $\mu$ mol phospholipid), and assuming that the head group area occupied on average by one phospholipid molecule is $a_{0}=0.825 \mathrm{~nm}^{2}$ (taking into account $a_{0}($ POPC $)=0.63 \mathrm{~nm}^{2}$ [19] and $a_{0}$ (cholesterol) $=0.39 \mathrm{~nm}^{2}$ [245]; molar ratio of phospholipid to cholesterol $=2: 1$ ), one $135 \mathrm{~nm}$-sized unilamellar vesicle consists of $\sim 1.3 \times 10^{5}$ phospholipid molecules $\left(=1.08 \cdot 10^{5} \mathrm{~nm}^{2} / 0.825 \mathrm{~nm}^{2}\right)$. Therefore, the estimated number of SOD molecules per vesicle is $\sim 3-4\left(=\left(2.83 \times 10^{-11}\right.\right.$ mol SOD molecules $/ 10^{-6}$ mol phospholipid molecules $) \times\left(1.3 \times 10^{5}\right.$ phospholipid molecules per vesicle $)=3.7$ SOD molecules per vesicle). The calculated trapped aqueous volume of one spherical vesicle with an inner diameter of $131 \mathrm{~nm}(=135 \mathrm{~nm}-4 \mathrm{~nm})$ is $\sim 1.07 \times 10^{-18}$ L. With this, the estimated average SOD concentration inside one vesicle for the experiments reported by Costa et al. (2020) [243] was $\sim 5 \mu \mathrm{M}$.

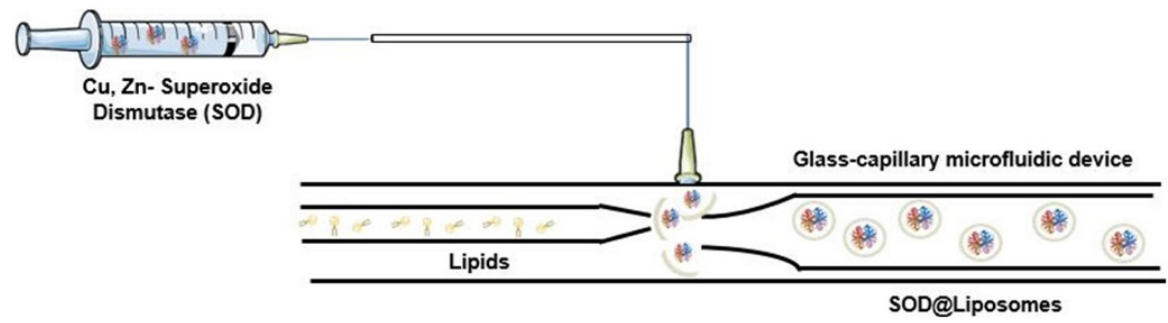

(a)

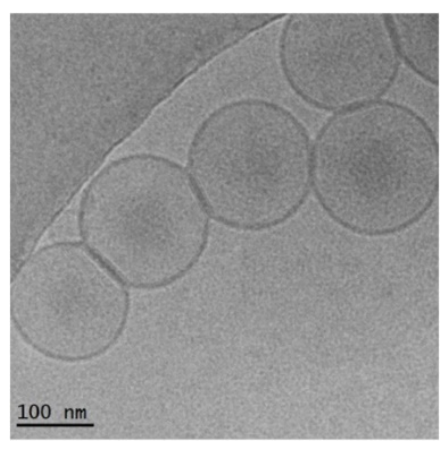

(b)

Figure 19. One-step microfluidics production of enzyme-loaded LUVs based on the "ethanol-injection method" by using a co-flow microfluidic glass capillary device, as published by Costa et al. (2021) [243]. (a) Schematic representation of the set-up. A borosilicate glass capillary ("inner" capillary) with an inner diameter of $100 \mu \mathrm{m}$ was placed coaxially in front of a "collecting" glass capillary with an inner diameter of $120 \mu \mathrm{m}$. Both capillaries were inserted into an "outer" capillary with an inner diameter of $1 \mathrm{~mm}$. A mixture of egg PC:cholesterol:DSPE-PEG2000 (1.85:1:0.15, molar ratio) dissolved in ethanol was injected into the "inner" capillary ("inner phase"), and an aqueous solution of the enzyme SOD (Cu,Zn-superoxide dismutase) was injected to the "outer" capillary ("outer phase"); see text and [243]. The size of the lipid vesicles obtainedSOD@Liposomes-for the experimental conditions used was $135 \pm 41 \mathrm{~nm}$, with efficient loading of the enzyme; see text and [243]. (b) Cryo TEM image of the SOD-containing vesicles obtained (bar: $100 \mathrm{~nm}$ ) [243]. Reproduced with permission from [243], Elsevier, 2021.

Although there are many other procedures that were (or are being developed) [193,246-252], the examples mentioned in Figure 17a should serve as illustration of some of the ways bilayer-forming amphiphiles can be guided to end up as vesicle dispersion consisting of spherical vesicles with a desired size of about $100 \mathrm{~nm}$ (LUVs).

The smallest unilamellar phospholipid vesicles that have been prepared were probably the ones reported by Zhigaltsev et al. (2016) [253]: 30-40 nm in diameter, composed of POPC/DPPC/DSPE-PEG2000 (45/20/35/3, molar ratio), prepared by using a modified 
"ethanol injection method". For drug delivery applications, LUVs with sizes of about $100 \mathrm{~nm}$ are often desired.

Each method has its advantages and disadvantages [191]. For some methods, the presence of remaining oil is an issue to consider, the possible "contamination" by nonbilayer-forming amphiphiles ("detergent depletion method"), the costs, or difficulties for an upscaling to larger sample volumes. Moreover, for the efficient encapsulation of water-soluble molecules during the vesicle preparation, some methods are more suitable ("reverse-phase evaporation method") than others ("ethanol injection method"); see also Chapter 5.

\subsection{Sophisticated Microfluidic Methods for the Formation of GUVs}

Recent progress in the field of microfluidics showed that for certain amphiphiles and under specific conditions, uniform GUVs can be obtained by microfluidic mixing on a specifically designed chip. In this case, mixing of two types of aqueous solutions (inner and outer) and a solution of amphiphiles in an oil occurs [254-263]; see Figure 20 [259,260,264].

Since an oil has to be used in such microfluidics-based procedures, the presence of remaining oil in the GUVs obtained needs to be considered, as in the case of GUVs prepared by the "droplet transfer method" (see above) or of GUVs prepared by jet-blowing [265]. With octanol as oil (in presence of $15 \mathrm{vol} \%$ glycerol), Deshpande et al. (2016) $[259,260]$ reported that conditions can be found for which octanol spontaneously separates as micrometer-sized droplets from the GUVs after initial $w / o / w$-double emulsion formation and that the presence of the amphiphilic block copolymer poloxamer $188, \mathrm{EO}_{76} \mathrm{PO}_{29} \mathrm{EO}_{76}$, was important for stabilizing the GUVs; see Figure 20 (bottom).

\subsection{Preparation of Vesicles with Asymmetric Membranes and/or with Internal Vesicles}

Independent from whether one aims at preparing LUVs or GUVs, details of the preparation methods are very important. Depending on the type of amphiphile used and on its chemical structure, or on whether complex amphiphile mixtures are used, optimization steps often are essential for a successful preparation. This is the engineering part of the vesicle preparation. It is as important as the scientific understanding of the general concepts of a certain preparation procedure.

There are various methods that can be applied for determining the "quality" of a prepared lipid vesicle dispersion, for example, by focusing on the average vesicle size and lamellarity, the surface charge, the chemical and colloidal stability, the amount of volume that is trapped by the vesicles in a certain total volume at a certain lipid concentration (see the legend of Figure 1), possible domain formation within the vesicle membrane (see Chapter 5), etc. [158,190,195,266,267].

When using different types of bilayer-forming amphiphiles, the preparation of vesicles, LUVs or GUVs, with a desired asymmetric distribution of the amphiphiles is also possible (Figure 21a). Such lipid asymmetry may stay for some time (a few days) until the energetically most favorable lipid distribution is obtained. The rate of equilibration depends on the fluidity of the membrane (i.e., $T_{\mathrm{m}}$ of the lipids) and the flip-flop rates for the amphiphiles within the bilayer [268]. The latter properties usually are a challenge to determine experimentally [118,269-272]. Among the methods depicted in Figure 17, asymmetric vesicles can be prepared by the "droplet transfer method" [194,228] and the "jet-blowing method" [235]. There are, however, also other methods that could be applied, for example, by means of a cyclodextrin-catalyzed phospholipid exchange [268,273-279].

Apart from the different procedures with which unilamellar-or mainly unilamellarvesicles can be obtained, there are also methods that allow the formation of multivesicular vesicles (MVVs), i.e., vesicles that contain internal non-concentrically arranged vesicles (Figure 21b). Although dispersions of MVVs (also called "vesosomes", [280]) can be obtained with some of the methods summarized in Figure 17, additional methods can also be used, usually applicable to a certain class of amphiphilic lipids; see the recent review by Giuliano et al. (2021) [281]. Depending on the method used, the inner vesicles may be 
composed of chemically different lipids than the outer "wrapping" vesicle, and MVVs with asymmetric membrane lipid distributions can be prepared as well.
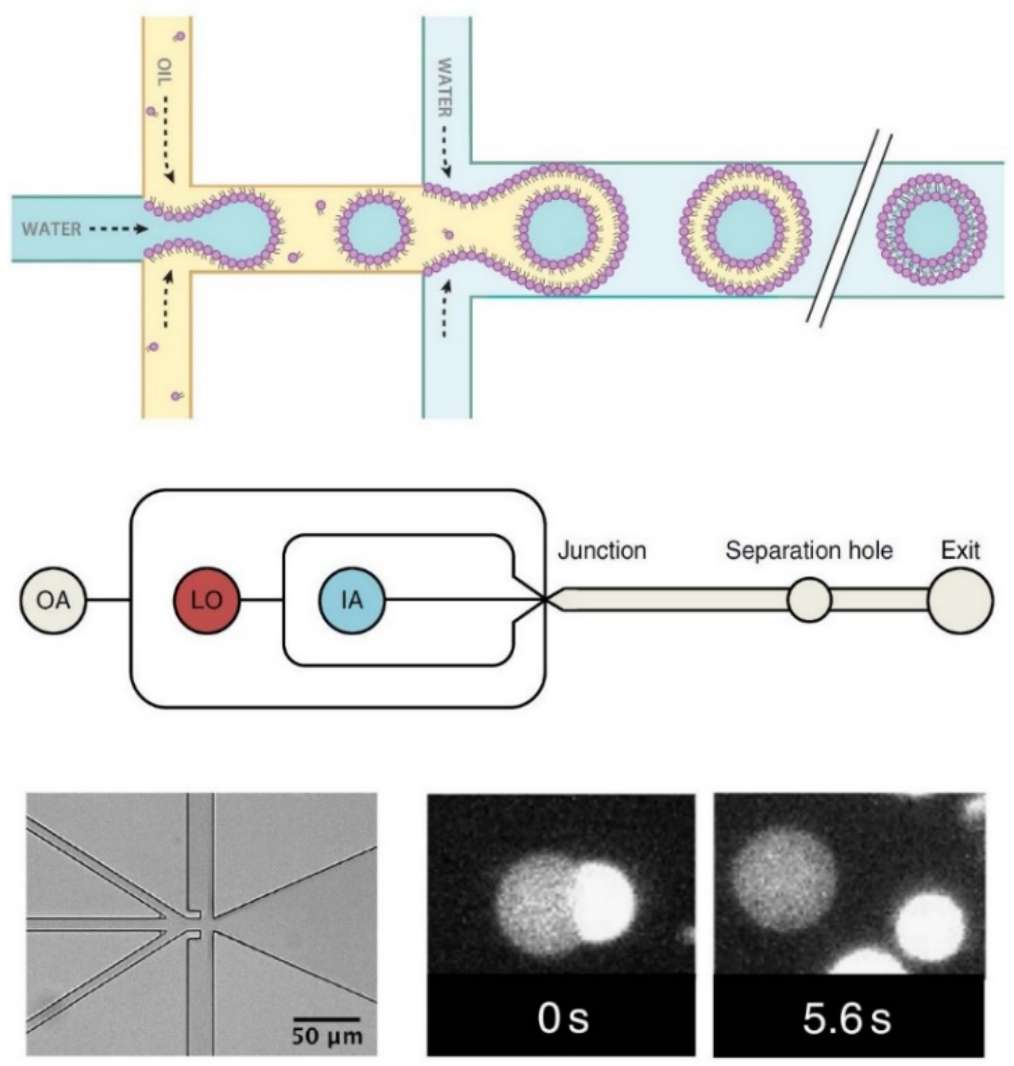

$20 \mu \mathrm{m}$

Figure 20. The use of microfluidics for the formation of GUVs. Top: Schematic representation of one of the microfluidics methods for the preparation of GUVs, as published by Litschel and Schwille (2021) [264]. The concept of the method is related to the conventional $w / o / w$-double emulsion method illustrated in Figure $17 \mathrm{~b}$. The key difference between the conventional method and the microfluidic method is that in the case of the microfluidic method the $w / o / w$-double emulsion is prepared in a very controlled way-droplet-by-droplet—on a microfluidic chip from an aqueous internal solution (dark blue "water"), an "oil" containing the amphiphiles (yellow "oil")—which finally form the membrane of the GUVs—and an external aqueous solution (light blue "water"). In contrast to the microfluidic LUV formation by the "ethanol injection method", where ethanol is used as water-miscible organic solvent (Figures 18 and 19), the "oil" used for the microfluidic GUV formation is a water-immiscible organic solvent (e.g., octanol in the presence of $15 \mathrm{vol} \%$ glycerol $[259,260])$. The initially formed $w / o / w$-droplets consist of an internal aqueous solution, an amphiphile-stabilized oil layer, and an external aqueous solution. As final step, the oil needs to be removed. Middle: Scheme of the chip used by Deshpande et al. (2018) [260], with OA, LO, and IA being the "outer aqueous solution", the "lipid-carrying oil", and the "inner aqueous solution", respectively. Bottom: Details of the chip (bar: $50 \mu \mathrm{m}$ ) [260] and fluorescence microscopy images (bar: $20 \mu \mathrm{m}$ ) showing the spontaneous removal of the "oil" octanol (originally containing $15 \mathrm{vol} . \%$ glycerol and $2 \mathrm{mg} / \mathrm{mL}$ DOPC with $0.1 \mathrm{~mol} \%$ of a fluorescently labelled PE) from the vesicles by a budding process that occurs due to interfacial forces acting on the droplets [259]. The octanol droplet (bright sphere) separates from a $w / o / w$-double emulsion droplet $(t=0 \mathrm{~s})$ and results in the formation of an almost octanolfree GUV and a separated octanol droplet (at $t=5.6 \mathrm{~s}$ ). The fluorescently labeled PE was DOPE-LissRhod, whereby "LissRhod" stands for the -(N-(lissamine rhodamine B sulfonyl moiety). The chip used was prepared from vinyl-terminated PDMS, poly(dimethylsiloxane), and the surface was coated with PVA, poly(vinylalcohol) to make it hydrophilic [259,260]. Reproduced from [264], copyright (C Annual Reviews, 2021; with permission from [259], Springer Nature, 2016; and from [260], Springer Nature, 2018. 


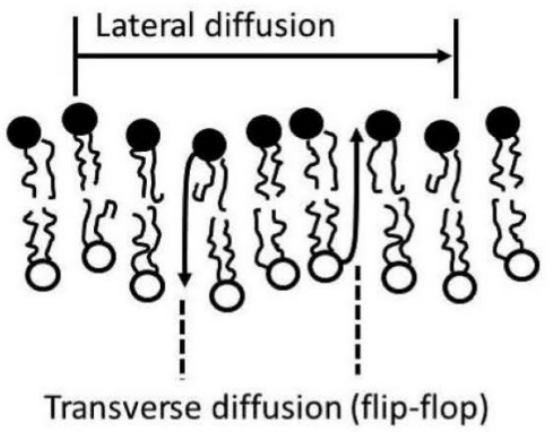

Asymmetric bilayer membrane

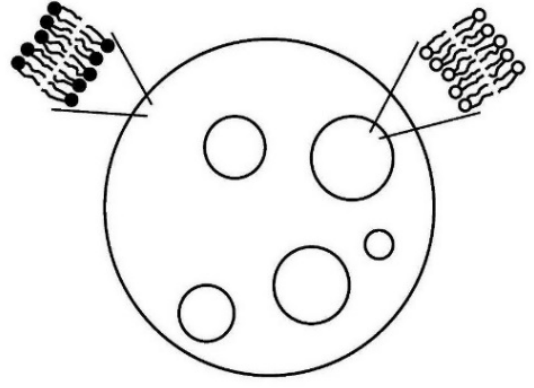

Multivesicular vesicles

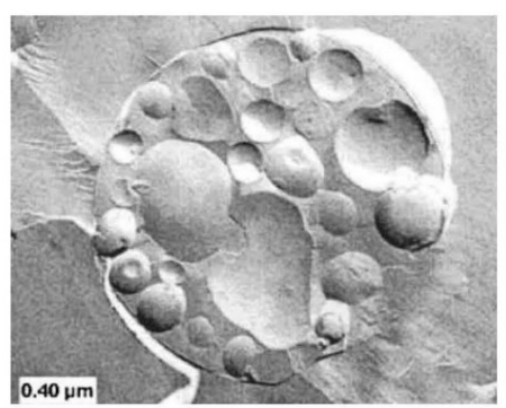

(a)

(b)

Figure 21. (a) Illustration of a section of a completely asymmetric fluid vesicle bilayer (with a high lateral lipid diffusion) built from a mixture of two different bilayer-forming amphiphiles (filled and empty polar head groups). Depending on Table 2019. [268]. (b) Freeze-fracture electron microscopy image of a multivesicular vesicle (MVV), consisting of an outer larger vesicle and many internal smaller vesicles (bar: $400 \mathrm{~nm}$ ). A dispersion of such MVVs was prepared by heating a dispersion of interdigitated sheets prepared from DPPC/cholesterol (97.5:2.5, molar ratio) to $T=46{ }^{\circ} \mathrm{C}$, as published by Kisak et al. (2002) [280]. Formation of the initial interdigitated sheet dispersion which occurred in the presence of $3 \mathrm{M}$ ethanol; see also Giuliano et al. (2021) [281]. Reproduced with permission form [268], American Chemical Society, 2019; and from [280], American Chemical Society, 2002.

\section{Functionalization of Lipid Vesicles and Possible Bicelle Formation \\ 5.1. Opportunities for the Functionalization of Lipid Vesicles}

Among the different polymolecular aggregates that amphiphilic lipids can form in aqueous solution, vesicles are unique.

- First of all, hydrophilic molecules can be entrapped in the interior aqueous volume of the vesicles, for example, low molar mass pharmaceutically active compounds or therapeutic enzymes. Such entrapment can be achieved during the vesicle preparation, followed by separation of non-entrapped compounds (see Chapter 4). In special cases, loading of the vesicles is also possible after vesicles formation (so-called "remote loading"); see Section 5.4. Independent from the way desired water-soluble molecules are entrapped inside vesicles of desired size, lamellarity, and membrane composition, the entrapped molecules are separated from the bulk solution by a lipid bilayer, which acts as protective permeability barrier for the entrapped molecules.

- Second, hydrophobic compounds can be embedded within the vesicle membrane. This can again be achieved either during vesicle preparation or after vesicle formation. Depending on the type of membrane-embedded compound, it may alter the physicochemical properties of the membrane in a desired way. The embedding of cholesterol into a fluid phospholipid bilayer, for example, can result in a significant bilayer rigidification, or it can lead to domain formation within the membrane due to a non-homogeneous distribution of the cholesterol and lipid molecules; see Section 5.3.

- Third, the external vesicle surface can be functionalized. Such surface functionalization can be achieved either during vesicle preparation by using amphiphiles with a desired functionalized head group (for example PEGylated lipids, as mentioned in Chapter 3, Figure 16b) or by chemically modifying the outer vesicle surface after vesicle formation.

Concerning the functionalization of the vesicle surface, there are many opportunities, depending on the desired application. Some of these opportunities towards pharmaceutical applications are summarized in Figure 22 [23]. Obviously, for a successful surface functionalization of vesicles, knowledge is required about the type and amount of functionalized amphiphiles that can be used and about the method of vesicle preparation that would be 
most suitable for a particular functionalization. This knowledge usually is gained from systematic investigations. Small changes in the chemical structure of the hydrophobic part of an amphiphile that one would like to incorporate in a vesicle bilayer and a variation of its content within the vesicle membrane may have a big effect on the properties of the vesicles.

A Conventional liposome Hydrophobic $_{1}$ D Theranostic liposome

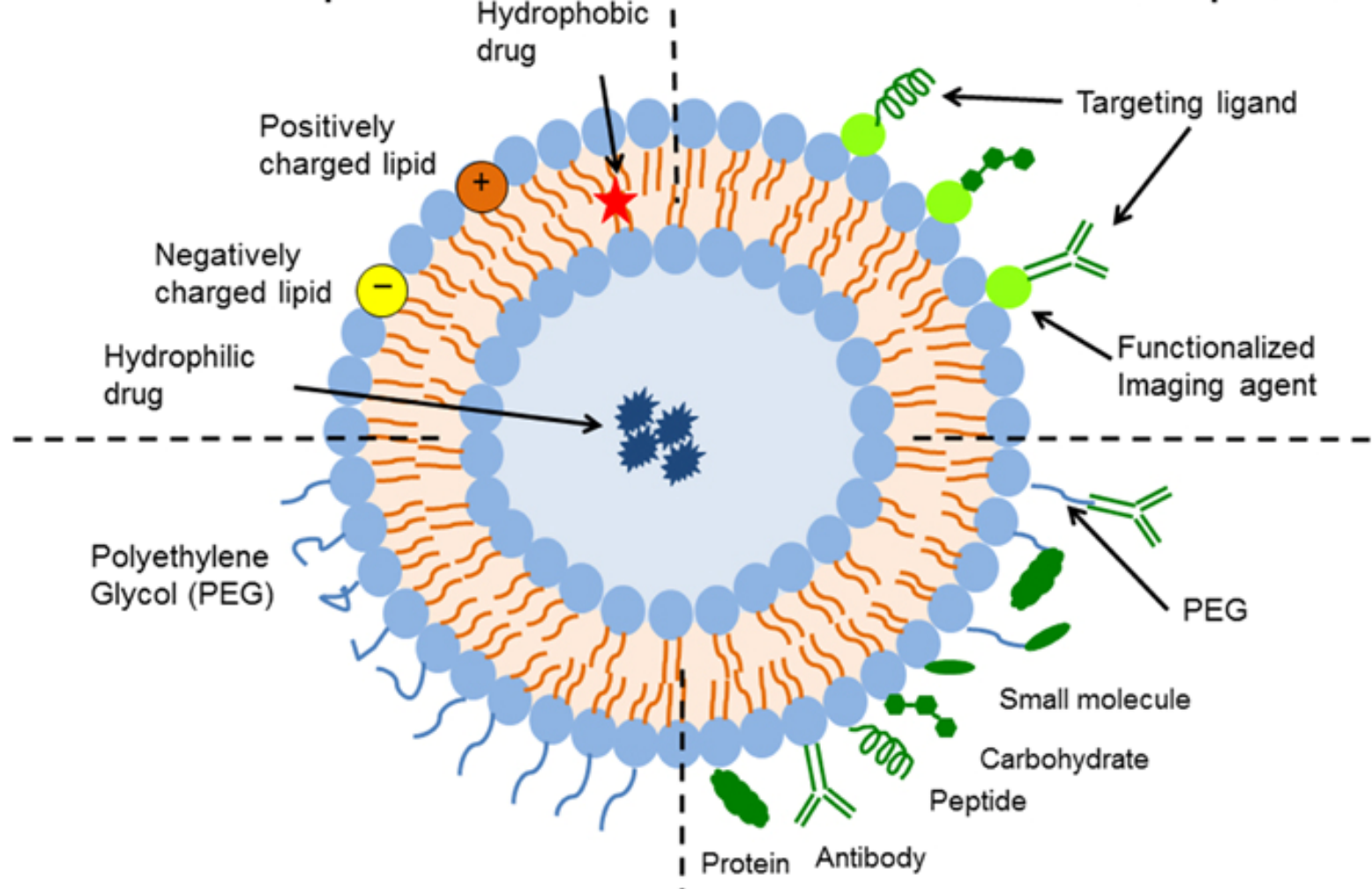

\section{B PEGylated liposome}

\section{Ligand-targeted liposome}

Figure 22. Schematic representations of a cross-section of an unilamellar vesicle with illustration of the possibilities for the functionalization of the vesicle surface for potential targeted drug delivery and theranostic applications, as published by Sercombe et al. (2015) [23]. For use as drug delivery systems, the possibilities for entrapping water-soluble (hydrophilic) and membrane-soluble (hydrophobic) drugs are also indicated. Other surface functionalities than the ones shown are also possible for non-pharmaceutical applications. The illustration is not drawn to scale as the diameter of the internal aqueous pool usually is considerably larger than the lipid bilayer thickness (often $\sim 90 \mathrm{~nm}$ diameter and 4-5 nm membrane thickness). The possibility surface PEGylation is also indicated; see Figure 16b. Reproduced from [23], Frontiers, 2015.

There are many examples of surface-functionalized vesicles. In addition to PEGylated vesicles (see Chapter 3), vesicles containing surface-bound immunoglobulins are wellknown for their potential use for targeted drug delivery [282-286].

\subsection{Vesicle Functionalization May Lead to the Formation of Bicelles}

Depending on the functionality one would like to place on a vesicle surface at a desired surface density, the formation of stable vesicles may not always be possible. One example is the case of an aqueous mixture of DMPC and DMPE-DTPA (1,2-dimyristoyl-sn-glycero3-phosphoethanolamine-diethylenetriaminepentaacetate), complexed to paramagnetic thulium ions $\left(\mathrm{Tm}^{3+}\right)$ at a molar ratio of 4:1:1 (DMPC:DMPE-DTPA:Tm${ }^{3+}$ ). Dispersing the lipids at a total concentration of $15 \mathrm{mM}$ at $p H=7.0$ in water (unbuffered, $p H$ adjusted by using $\mathrm{NaOH}$ ), followed by polycarbonate membrane extrusion using for final extrusions membranes with a pore diameter of $100 \mathrm{~nm}$, results in the formation of "bicelles", disk-like aggregates at $T=5-30{ }^{\circ} \mathrm{C}$ with a disk diameter of about $40 \mathrm{~nm}$ and a thickness of about 
$4 \mathrm{~nm}[287,288]$ (Figure 23a). Although the dominating lipid DMPC is a bilayer forming amphiphile, vesicles do not form at $T=25^{\circ} \mathrm{C}$ under the conditions used. The polar DTPA$\mathrm{Tm}^{3+}$ head group is too large for accommodating all DMPE-DTPA-Tm ${ }^{3+}$ amphiphiles in the DMPC bilayer. DMPE-DTPA-Tm ${ }^{3+}$ is a micelle-forming amphiphile and occupies preferentially the edges when mixed with DMPC under the chosen conditions at which the DMPC molecules tend to form bilayers in a solid-ordered $\left(s_{0}\right)$ state. Therefore, the two amphiphiles demix and form separate domains, flat bilayers (rich in DMPC molecules) and highly curved "semi-micelles" (rich in DMPE-DTPA-Tm ${ }^{3+}$ ). The inability of vesicle formation for this particular lipid mixture was first discovered after it was found to be impossible to entrap in the aggregates that formed a water-soluble fluorescent dye (calcein). Simply judging from the translucent appearance of the samples prepared in the absence of calcein (similarly to the appearance in the case of extruded POPC vesicle dispersions, for example), one might be tempted to conclude-erroneously_that vesicles formed. A careful cryo-TEM analysis, however, clearly showed that bicelles instead of vesicles were obtained (Figure 23a). The formation of bicelles was in agreement with the simple dye entrapment experiments and was further supported by detailed small angle neutron scattering (SANS) measurements $[287,288]$. This example highlights the importance of the use of some of the many analytical methods that are available for characterizing lipid vesicles and other polymolecular aggregates to unambiguously prove the formation of vesicles if one aims to prepare them. For the mentioned aqueous DMPC/DMPE-DTPA-Tm ${ }^{3+}$ system, vesicle formation can still occur at $T>35^{\circ} \mathrm{C}$, above $T_{\mathrm{m}}$ of DMPC $\left(23.6 \pm 1.5^{\circ} \mathrm{C}\right.$ [101]), or at $T=25^{\circ} \mathrm{C}$ if DMPC is replaced by POPC $\left(T_{\mathrm{m}}=-2.5 \pm 2.4{ }^{\circ} \mathrm{C}\right.$ [101]) [289,290] (Figure 23b). This showcases how seemingly minor changes in the chemical structure of the amphiphiles used can influence their aggregation behavior due to the dependence of the physicochemical properties of the amphiphiles on their chemical structure [291]. The high mobility of the lipids in the fluid state of POPC at $T=25^{\circ} \mathrm{C}$ allows accommodating DMPE-DTPA$\mathrm{Tm}^{3+}$ within the bilayer to form self-closed, curved bilayers (vesicles) and not bicelles.

Probably the most intensively investigated bicelles are the ones composed of DMPC and 1,2-dihexanoyl-sn-glycero-3-phosphocholine, DHPC [292-295]. Depending on the total concentration of DMPC and DHPC, on the molar DMPC:DHPC ratio, and on the temperature, bicelles form or (perforated) multilamellar vesicles. In the case of bicelles, the sort of micelle-forming DHPC molecules are localized preferentially at the edges [292,294], just like DMPE-DTPA-Tm ${ }^{3+}$ in the case mentioned above and illustrated in Figure 23b (top).

\subsection{Domain formation within Vesicle Membranes May Occur}

Domain formation within the bilayers of intact vesicles is well-known for phospholipid/cholesterol mixtures, although the size (ranging from a few nanometers to several micrometers) and dynamics of the domains is very complex and depends on many factors, such as type of phospholipid (phosphatidylcholines, sphingomyelins), cholesterol content, and temperature [296-312]. As shown in Figure 24, diagrams for illustrating the state of the bilayers in aqueous mixtures of phosphatidylcholines and cholesterol, as examples, were determined [297-300,302,305]. The presence of cholesterol can lead to the formation of a liquid-ordered $\left(l_{\mathrm{o}}\right)$ state and to the coexistence within the same membrane of $l_{\mathrm{d}}$ and $l_{\mathrm{o}}$ or $l_{\mathrm{d}}$ and $s_{\mathrm{o}}$ states. The $l_{\mathrm{o}}$ state is characterized by a reduced head group hydration as compared to the $l_{\mathrm{d}}$ state, as determined for mixed DPPC/sphingomyelin bilayers using fluorescent membrane probes [300]. 

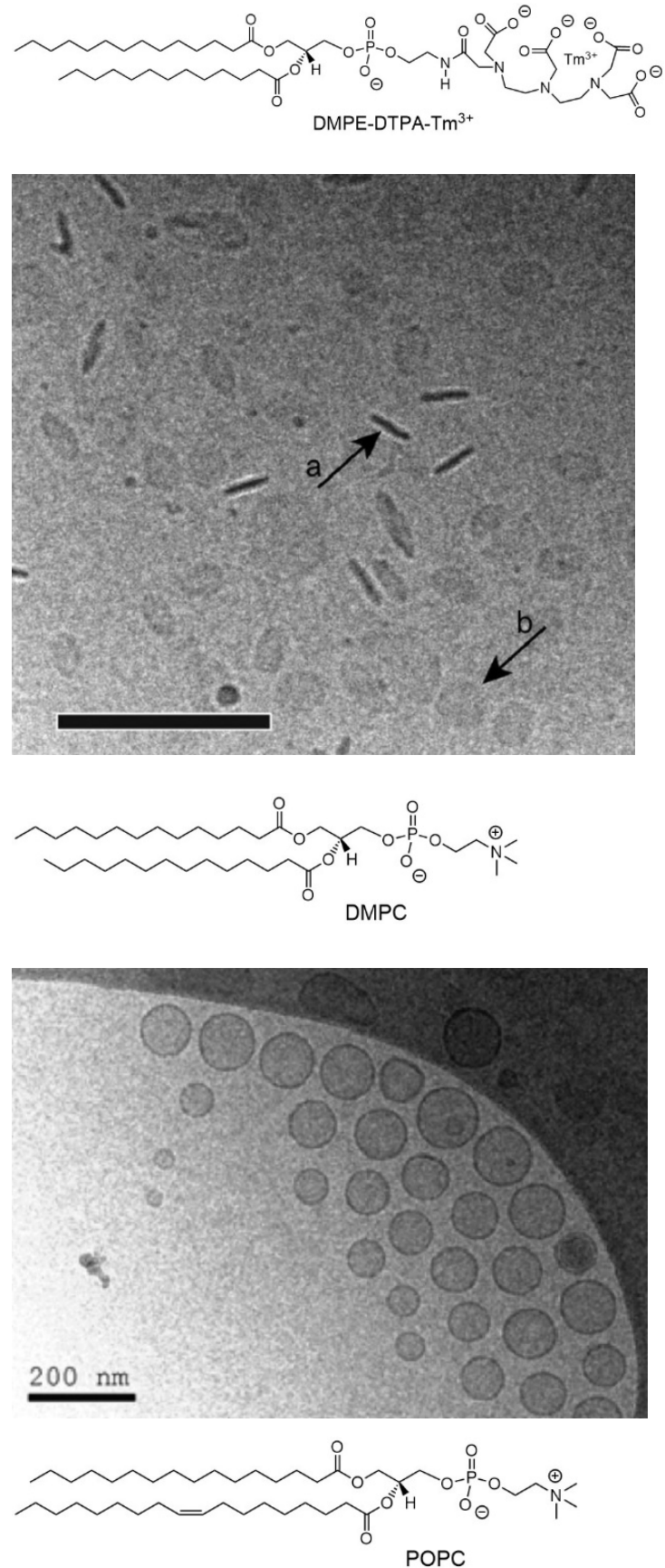

(a)
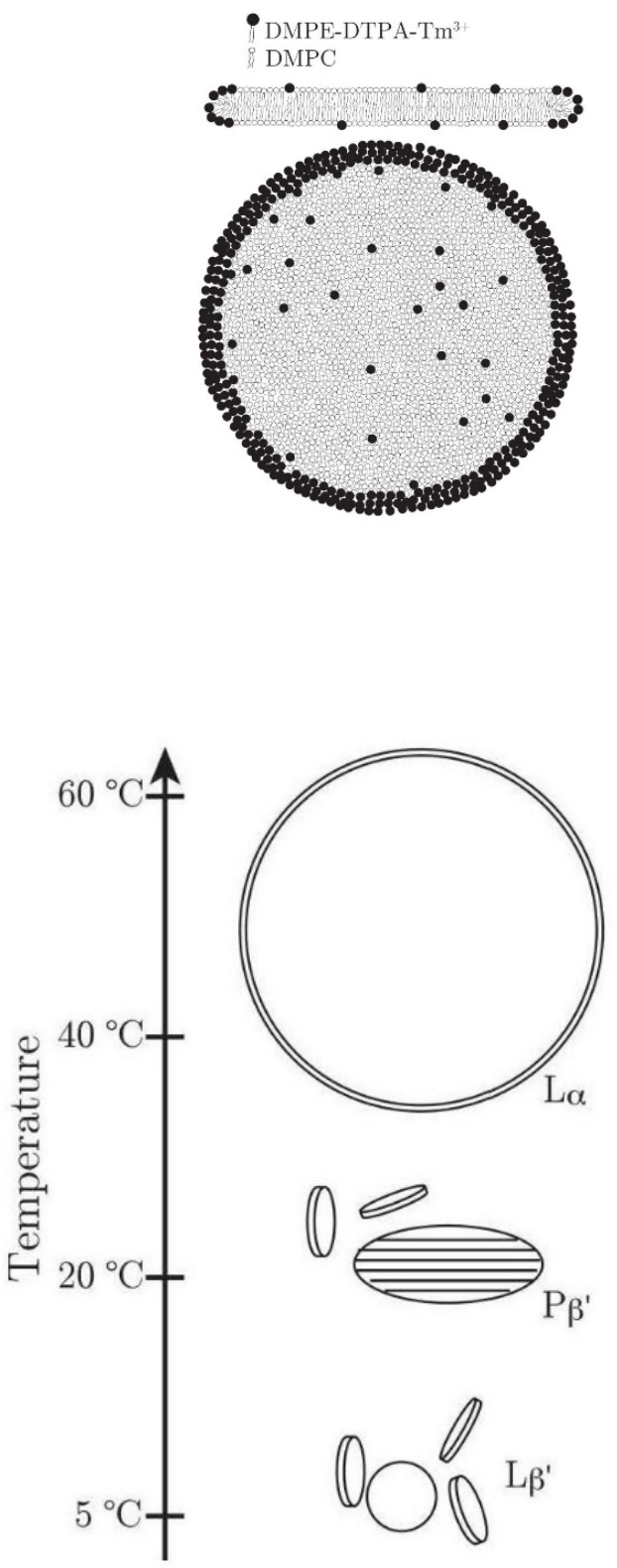

Vesicle

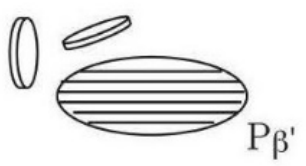

Disk

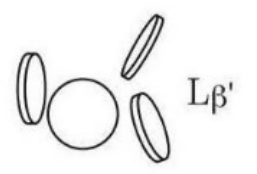

Disk

(b)

Figure 23. Formation of bicelles or vesicles. (a) Top: Cryo-TEM image of an aqueous dispersion of bicelles formed from an aqueous DPMC:DMPE-DTPA:Tm ${ }^{3+}$ (4:1:1, molar ratio) mixture (total lipid concentration: $\left.15 \mathrm{mM}\right)$, prepared by polycarbonate membrane extrusion with final extrusion through $100 \mathrm{~nm}$-sized pores, frozen from $T=5{ }^{\circ} \mathrm{C}$ (bar: $200 \mathrm{~nm}$ ); see text. The two arrows point to a bicelle lying edge-on (a) and one lying face-on (b) [287]. Bottom: Cryo-TEM image of an aqueous dispersion of vesicles formed from an aqueous POPC:DMPE-DTPA:Tm ${ }^{3+}$ (4:1:1, molar ratio) mixture, prepared by polycarbonate membrane extrusion with final extrusion through $100 \mathrm{~nm}$-sized pores, frozen from $T=5{ }^{\circ} \mathrm{C}(\mathrm{bar}: 200 \mathrm{~nm})$ [289]. (b) Top: Schematic representation of a 4:1:1 DPMC:DMPE-DTPA:Tm ${ }^{3+}$ bicelle, indicating the non-homogenous distribution of the two amphiphiles within the bicelle. Bottom: Temperature dependence of the aggregates formed in aqueous dispersions of DPMC:DMPE-DTPA:Tm ${ }^{3+}$ (4:1:1, molar ratio), at a total lipid concentration of $15 \mathrm{mM}$ [288]. DPMC:DMPE-DTPA:Tm ${ }^{3+}$ is 1,2-dimyristoyl-sn-glycero-3-phosphoethanolamine-diethylenetriaminepentaacetate, complexed to a paramagnetic thulium ion $\left(\mathrm{Tm}^{3+}\right)$. Reproduced with permission from [287], American Chemical Society, 2010; from [289], American Chemical Society, 2010; and from [288], ETH Zürich, 2013. 

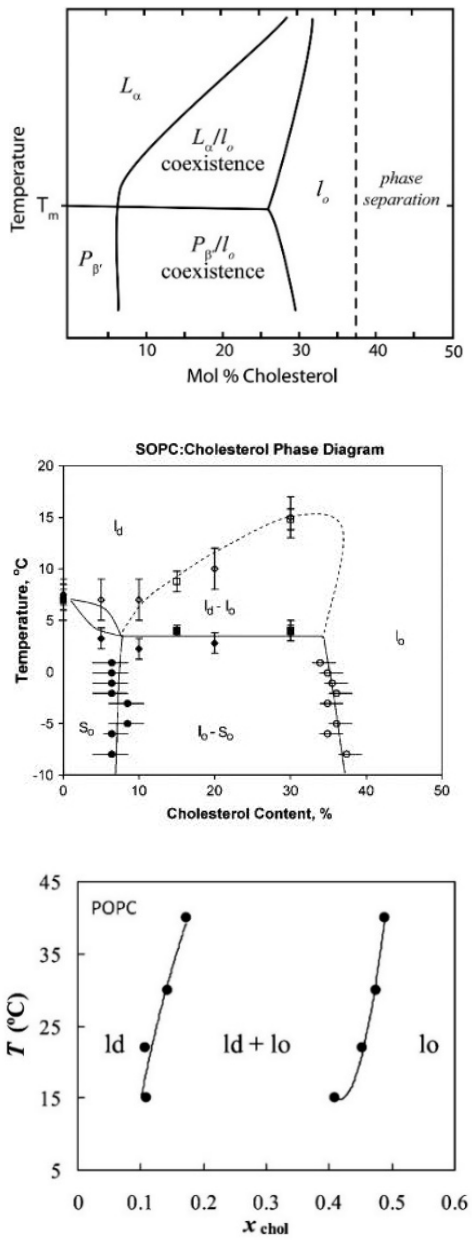

(a)
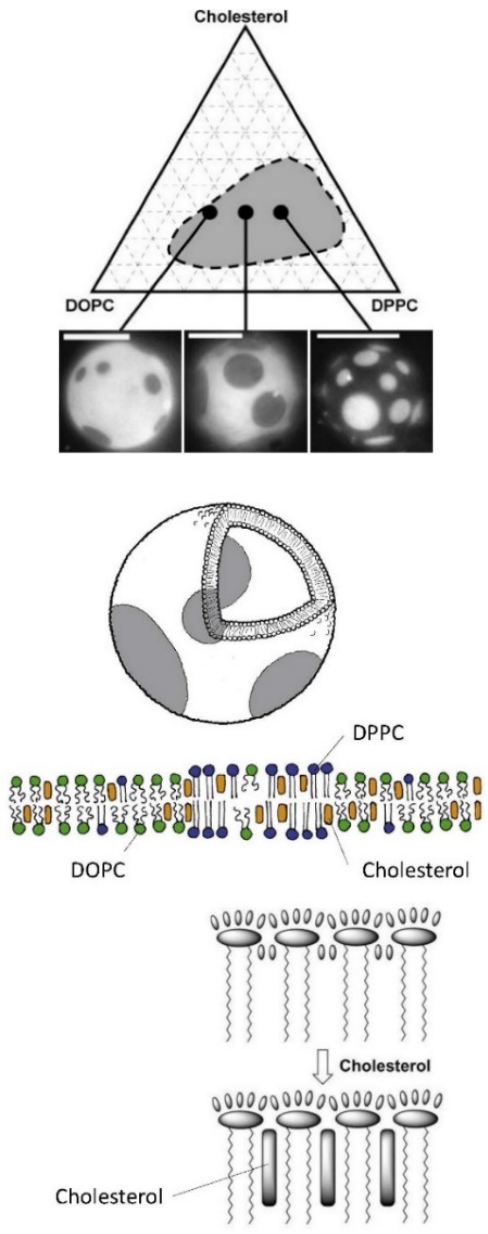

(b)

Figure 24. Domain formation in mixed aqueous phospholipid/cholesterol bilayers (a) Top: Generalized bilayer state diagram for bilayers of phosphatidylcholines and cholesterol as a function of cholesterol content and temperature, as published by Rheinstädter et al. (2013) [305]. Depending on the cholesterol content and the temperature, single states exist within the bilayer $\left(L_{\alpha}\right.$ at $T>T_{\mathrm{m}}, P_{\beta^{\prime}}$ at $T<T_{\mathrm{m}}$, both at low cholesterol contents; $l_{\mathrm{o}}$ at $\sim 30-37 \mathrm{~mol} \%$ cholesterol; or coexisting states are present $\left(L_{\alpha}\right.$ and $l_{\mathrm{o}}$, or $P_{\beta^{\prime}}$ and $l_{\mathrm{o}}$ ). At high levels of cholesterol, cholesterol separates as pure crystalline phase [305]. Middle: Elaborated diagram for illustrating the situation in the case of aqueous SOPC/cholesterol bilayers, as determined by differential scanning calorimetry/DSC) $(\downarrow, \diamond)$ proton magic angle spinning NMR $(\boldsymbol{\square}, \square)$, and deuterium NMR $(\bullet, \bigcirc)$ measurements, as published by Polozov and Gawrisch (2006) [299]. SOPC is 1-stearoyl-2-oleoylsn-glycero-3-phosphocholine, $T_{\mathrm{m}}(\mathrm{SOPC})=6.9 \pm 2.9^{\circ} \mathrm{C}$ [101]. Bottom: Elaborated diagram for aqueous $\mathrm{POPC} /$ cholesterol bilayers, as determined from measurements of the steady state anisotropy of embedded DPH (1,6-diphenylhexatriene) and fluorescence lifetime measurements of $t$-PnA (trans-parinaric acid), as published by de Almeida et al. (2003) [297]. (b) Top: Triangular representation of the domain formation in mixed DOPC/DPPC/cholesterol bilayers at $T=25^{\circ} \mathrm{C}$, determined by analyzing fluorescence micrographs using DPPE-Texas Red as fluorescent probe that partitions preferentially into less ordered, liquid domains; see Veatch et al. (2004) [298]. The GUVs shown were prepared by "electroformation" at 30\% cholesterol and the following compositions (from left to right): DOPC/DPPC (2:1), DOPC/DPPC (1:1), and DOPC/DPPC (1:2) (bars: $20 \mu \mathrm{m})$ [298]. Middle: Schematic drawing of unilamellar vesicle and illustration of two liquid states containing different fractions of the three lipid types; see Honerkamp-Smith et al. (2009) [302]. Bottom: Schematic model of the effect of cholesterol (long gray bars) on the presence of water molecules (small gray ellipsoids) near the phospholipid head groups forming the $l_{\mathrm{o}}$ state within the bilayer; see M'Baye et al. (2008) [300]. Reproduced with permission from [305] Elsevier, 2013; from [299], Elsevier 2006; from [297], Elsevier, 2003; from [296], Elsevier, 2004; from [302], Elsevier, 2009; and from [300], Elsevier, 2008. 
GUVs are particularly useful for visualizing the formation of stable, micrometer-sized domains. Such domain visualization can be achieved by using a fluorescently labeled lipid which partitions preferentially into less ordered, i.e., more fluid, domains, as compared to more ordered and therefore less fluid ones [296,298] (Figure 24b, top). Alternatively, two different types of fluorescent amphiphilic lipids can be applied as probe molecules [313] which (i) differ in the physical properties of the hydrophobic chains, and (ii) have different fluorescent moieties in the polar head group [306,313]. Due to the differences in the hydrophobic chain properties, the two fluorescently labeled lipid partition selectively into the $l_{\mathrm{d}}$ or $l_{\mathrm{o}}$ domains.

Concerning the embedding of membrane-soluble compounds within the vesicle membrane in general, the maximum amount that can be embedded depends on the chemical structure of the vesicle membrane-forming amphiphiles and on the chemical structure of the compounds that one likes to embed. A too high amount is expected to result in a destabilization of the vesicles. From a practical point of view, membrane-soluble molecules can be added at the beginning of the vesicle preparation, for example, admixed to the solution of bilayer-forming lipids with which a dried thin film is first formed. Alternatively, membrane-soluble compounds may also be added to pre-formed fluid vesicles [314].

\subsection{The "Remote Loading" of Lipid Vesicles with Certain Water-Soluble Compounds}

For many applications, the entrapment of water-soluble compounds within the aqueous interior is required; see Chapter 6. For such entrapment, the vesicles can be prepared by using an aqueous solution containing the molecules to be entrapped for the vesicle preparation [189]. Depending on the vesicle preparation method, the entrapment may not be very efficient, for example, in the case of the "ethanol injection method" [212,213], unless the method is optimized accordingly [315]. In any case, non-entrapped molecules need to be removed after vesicle preparation, for example, by ultracentrifugation or size exclusion chromatography [20]. High entrapment yields are possible by using the "reverse phase evaporation method" [208], the "droplet transfer method [228], the "lipid-coated ice droplet hydration method" [232,316], or the "dehydration-rehydration method" [191,317,318]. In the latter case, the molecules to be entrapped inside the vesicles are forced to come in close contact to the bilayer-forming lipids when an initially formed vesicle dispersion is dehydrated (removal of water molecules only, for example, by freeze-drying), before rehydration with water molecules is initiated [317].

The efficient loading of vesicles with water-soluble molecules often is a big challenge and a bottle-neck for desired applications. The development of the "remote loading methodology" for the preparation of doxorubicin-containing vesicles [198,319-329] can be considered as one of the two breakthroughs for the application of vesicles as drug delivery systems. The second breakthrough was the development of sterically stabilized, PEGylated liposomes (Figure 16b).

The "remote loading" (also called "active loading") of phospholipid vesicles with doxorubicin (also known under the brand name adriamycin) occurs after vesicle formation by using a $\mathrm{pH}$ and ammonium sulfate gradient across the vesicle membrane. At the applied external alkaline $\mathrm{pH}$, the majority of the added doxorubicin molecules is uncharged and able to penetrate across the vesicle membrane. At the low $\mathrm{pH}$-value inside ( $p H<5.25$ - - achieved by buffer species that do not permeate the membrane easily (citric acid/citrate)—doxorubicin is getting protonated (i.e., charged) and crystallizes (i.e., forms fibrous precipitates) in the presence of entrapped ammonium sulfate; see Figure 25. With this, high internal concentrations of doxorubicin can be achieved, important for the use as a vesicle-based drug delivery system, the first liposomal system that was approved 1995 by the American Food and Drug Administration (FDA). The product is known under the trade name Doxil ${ }^{\circledR}$, a dispersion of PEGylated phospholipid vesicles; see Section 6.2.1. 
<smiles>COc1cccc2c1C(=O)c1c(O)c3c(c(O)c1C2=O)C[C@@](O)(C(=O)CO)C[C@H]3O[C@H]1C[C@H](N)[C@@H](O)[C@H](C)O1</smiles>
$\left(\mathrm{DOX}-\mathrm{NH}_{2}\right)$

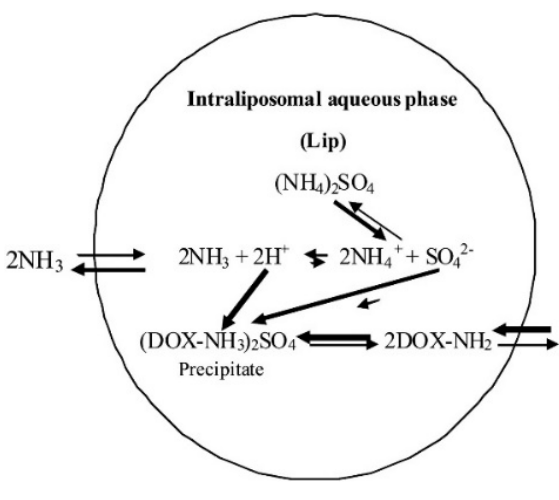

(a)
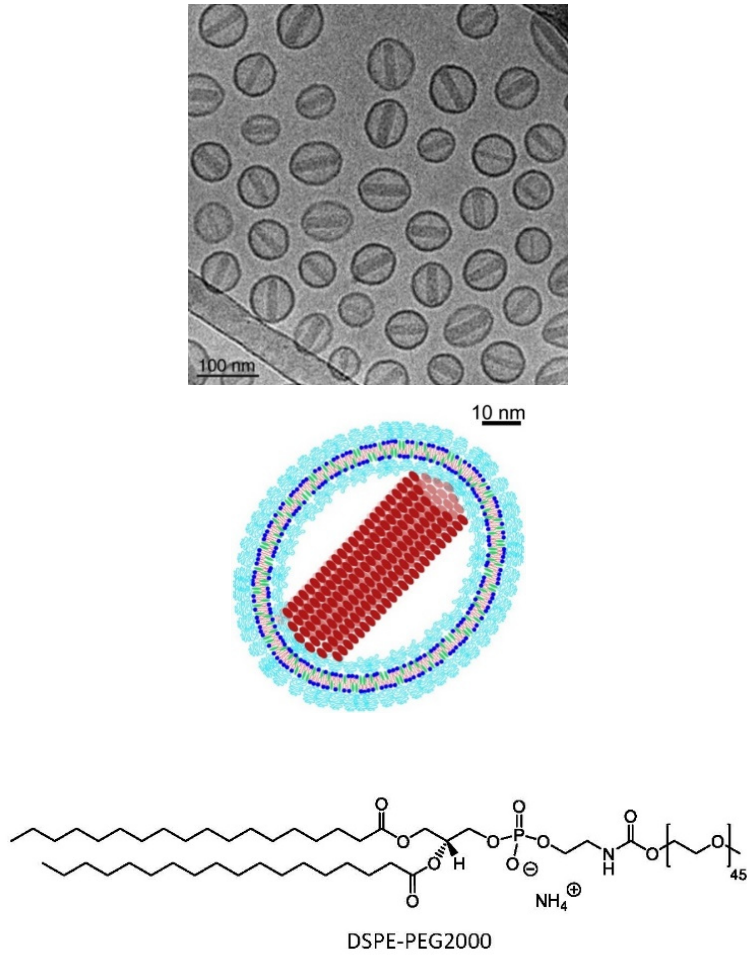

(Med)

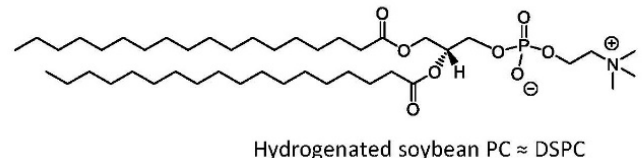

\section{$\uparrow \downarrow$} $2 \mathrm{DOX}-\mathrm{NH}_{2}+2 \mathrm{H}^{+}$

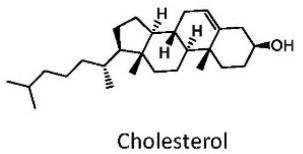

(b)

Figure 25. Doxil ${ }^{\circledR}$, as prepared by using the "remote loading method". (a) Top: Chemical structure of doxorubicin $\left(\right.$ DOX- $\left.\mathrm{NH}_{2}\right)$. The reported $\mathrm{pK}_{\mathrm{a}}$-value of the protonated form of the amine $\left(-\mathrm{NH}_{3}{ }^{+}\right)$of doxorubicin is 8.15 [323]. Bottom: Schematic representation of the remote loading of doxorubicin into pre-formed phospholipid vesicles. The loading is based on trans-membrane ammonium sulfate and pH-gradients, as published by Barenholz (2001) and (2012) [322,324]. The circle represents a phospholipid bilayer of a LUV, for example prepared by polycarbonate membrane extrusion; see Section 4.1. [323]. The arrows in bold indicate the situation during the loading process; see [324] and text for details. (b) Top: Cryo-TEM image of commercial Doxil ${ }^{\circledR}$, consisting of PEGylated phospholipid vesicles and internal doxorubicin precipitates, obtained via the remote loading mechanism shown in (a); see [324]. Middle: Illustration of the structure of a Doxil ${ }^{\circledR}$ product vesicle (not drawn to scale), consisting of internal doxorubicin precipitates and a single bilayer composed of HSPC (hydrogenated soybean phosphatidylcholine), DSPE-PEG2000, and cholesterol [329] (bottom); see Section 6.2.1. Reproduced with permission from [324], Elsevier, 2012; and from [329], Elsevier, 2016.

\section{Selected Examples of Applications of Lipid Vesicles and Related Lipid Aggregates}

\subsection{Overview}

There are many different research areas where lipid vesicles (liposomes) are either successfully applied as soft compartment systems or where fundamental studies are carried out towards real applications [186,330,331]; see Figure 26. The types of application can be divided into two groups. Applications of the first group are in areas where a commercialization of a liposomal product is the target (e.g., for drug delivery or as cosmetic product). Applications of the second group deal with lipid vesicles as tools for research works in 
academia, for example, as model system for gaining insight into the organization and properties of the plasma membrane of biological cells. Most of the applications belonging to the first group are driven by creative and innovative ideas as part of fundamental investigations - often without any direct involvement of the researchers in a translation of the idea into a "real world application" — there are several commercial liposome-based products that currently are (or previously were) in commercial use, either for the delivery of drugs [21-23,138,332-336], as vaccines [336-340], or as cosmetic products [330,341-344].

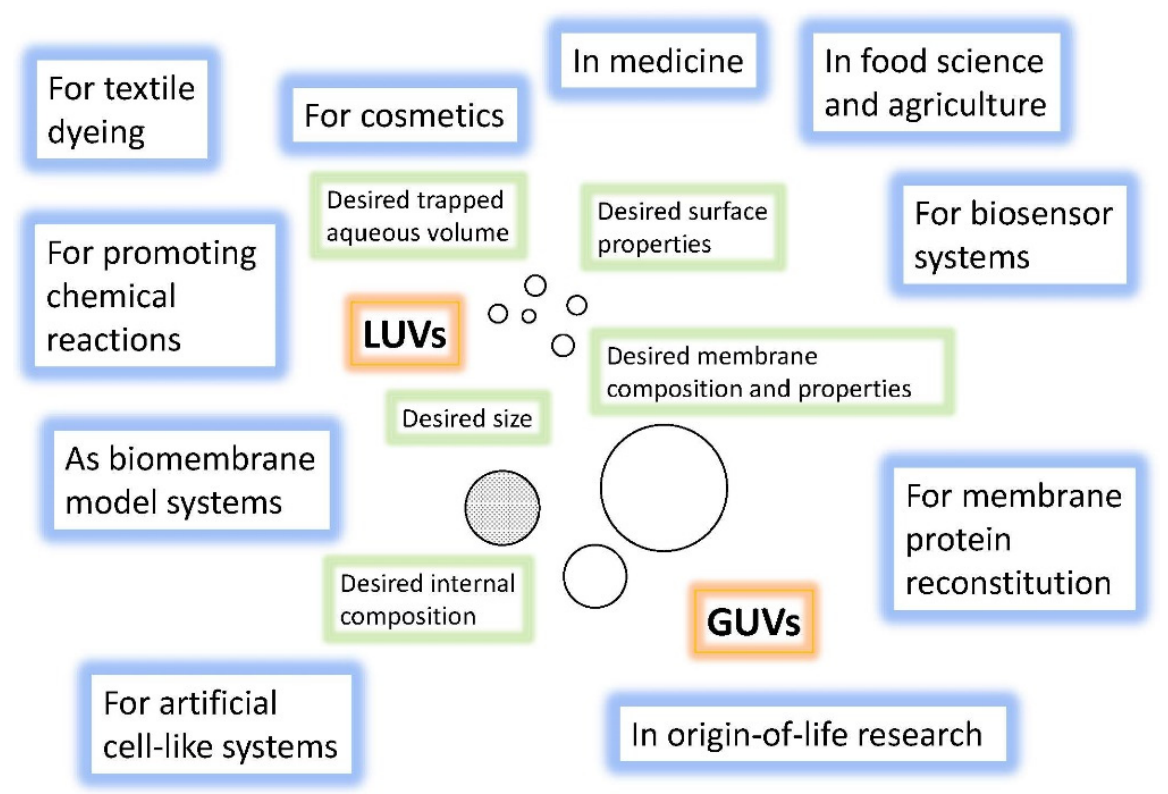

Figure 26. Some of the areas where lipid vesicles are used, either in commercial products, for preclinical or clinical studies (as LUV dispersions), or as tools for fundamental studies in research laboratories (LUVs or GUVs). Depending on the application, the size and properties of the vesicles can be tuned; see Chapters 4 and 5 .

There are at least five reasons to explain why so many application-oriented studies involving lipid vesicles were (or are being) published, often ending with the conclusion that the results obtained with the particular vesicular systems used are (very) promising:

- The good level of understanding of the correlation between chemical structure of an amphiphile and types of aggregates that can be formed in an aqueous medium and of the aggregates' thermodynamic and kinetic properties.

- The remarkable size range within which lipid vesicles can be prepared, from $\sim 30 \mathrm{~nm}$ to $100 \mu \mathrm{m}$ (and more), and the knowledge about the concepts and methods that have been elaborated for obtaining stable vesicles with a relatively defined diameter (preparation methods).

- The knowledge about the concepts that can be used for loading vesicles with desired water- and/or membrane-soluble ingredients.

- The possibilities of tuning lipid vesicles, basically at will, by incorporating functionalized, partially synthetic amphiphiles in the membrane, or by modifying some of the vesicle-forming amphiphiles present in the membrane after vesicle formation.

- The fact that many of the lipid vesicles prepared with biological lipids as main membrane-forming amphiphiles are biocompatible, biodegradable and toxicologically safe [138,335].

Despite these advantages of lipid vesicles, there are also considerable challenges that need to be considered if one likes to move towards a commercialization:

- The expected costs for the development of a product might be unacceptably high due to the molecular complexity of specifically engineered vesicles and the method of vesicle preparation and purification, as compared to the expected quantity that 
one would need to produce and the price consumers would be willing to pay for a specific product.

- The loading of the vesicles with desired active compounds might not be as efficient as required.

- The desired long-term storage stability of a liposomal product in dried, lyophilized state and the reconstitution of the liposomes after rehydration might not always be easy to achieve [345,346].

For medical applications, these three considerations-among others-often hinder undertaking steps to move from laboratory investigations to preclinical studies and to proceed from successful preclinical investigations to clinical trials [138,333,335,347]. There are, however, also other areas where either sophisticated vesicles are not needed (e.g., for cosmetics), or a mass production of vesicles is not required. An example of the latter case is the non-commercial laboratory application of lipid vesicles as model systems for investigating the physics of biological membranes [181,182,184,348-353] or in origin-of-life research where lipid vesicles are used as one of the model systems of the hypothetical precursor compartment structures ("protocells") that may have preceded the first cells [35,354-363].

In the following, some of the areas where lipid vesicles are applied are briefly mentioned, together with a few selected examples to illustrate some of the recent developments. What is not included here are the many efforts on developing vesicular systems from fully synthetic, largely non-natural compounds, including vesicles from non-ionic, low molar mass amphiphiles (also called "niosomes") [364-366], vesicles from synthetic anionic [367-371] or cationic [367,368,371-375] low molar mass amphiphiles-or mixtures therefrom [374-378]—or "polymersomes", i.e., vesicles from amphiphilic block copolymers (or block cooligomers) [379-385]. Some of these synthetic vesicle systems yielded promising results in research laboratories, for example, as enzyme-containing reactor units for potential in vivo applications as artificial organelles [386,387], as additives for guiding chemical or enzymatic reactions $[369,370,388,389]$, as versatile sensors based on polydiacetylene vesicles [390-394], etc. Although artificial vesicles are used in cosmetic products [364,390], a translation of polymersome-based drug delivery systems, for example, into "real world applications" has not been achieved yet [395].

\subsection{Liposome (and "Lipid Nanoparticle") Applications in Medicine \\ 6.2.1. Doxil ${ }^{\circledR}$, AmBisome $^{\circledR}$, Visudyne ${ }^{\circledR}$, Exparel $^{\circledR}$, and Inflexal ${ }^{\circledR} \mathrm{V}$}

There are large numbers of investigations on the (potential) use of lipid vesicles in various medical areas, for therapeutic, prophylactic or diagnostic applications. Studies on liposomal drug delivery dominate, mainly for the treatment of cancer via the delivery of liposome-encapsulated anti-tumor drugs to tumor cells for inhibiting their growth. In successful cases, encapsulation of anti-tumor drugs within the liposomes results in a lower drug toxicity, as compared to the free drug, as well as to a prolongation of the time the drug stays in the blood circulation after intravenous (i.v.) administration, if the liposomes are sterically stabilized with a surface bound water-soluble polymer (use of "stealth liposomes"; see Figure 16b). The improvement of the toxicity profile of a liposomal anti-cancer drug, as compared to the free drug, yields a better patient compliance and quality of life [396].

As already mentioned above, several liposomal products currently are in clinical use, most of them for i.v. administrations [332-335]. Well known is Doxil ${ }^{\circledR}$ (Figure 25b), with doxorubicin as active agent and HSPC (hydrogenated soybean phosphatidylcholine): cholesterol:DSPE-PEG2000 (56.51:38.18:5.31, molar ratio) as liposome membrane constituents, $75-90 \mathrm{~nm}$ mean size) [25,329,332]. Hydrogenation of soybean PC eliminates the cis-double bonds present in the PC mixture isolated from soybeans (Table 2), which results in an increase in $T_{\mathrm{m}}$, from below $0{ }^{\circ} \mathrm{C}$ for soybean $\mathrm{PC}$ (see Section 2.11) to $T_{\mathrm{m}} \sim 54{ }^{\circ} \mathrm{C}$ for HSPC [397] (similar to $T_{\mathrm{m}}(\mathrm{DSPC})=54.5 \pm 1.5^{\circ} \mathrm{C}$ [101]). The presence of cholesterol influences the state of the lipids within the bilayer and the formation of domains, depending on the cholesterol content [397]; see also 5.3. Each liposome in Doxil ${ }^{\circledR}$ contains 
10,000-15,000 doxorubicin molecules [25], corresponding to an overall intraliposomal concentration of about $45 \mathrm{mM}$, present as fibrous precipitates. Liposomal systems for other therapies than anti-cancer were investigated as well, e.g., (i) for the treatment of fungal infections (AmBisome ${ }^{\circledR}$ (i.v.), containing amphothericin B as active agent, with HSPC:DSPG:cholesterol:amphotericin B (2:0.8:1:0.4, molar ratio), $100 \mathrm{~nm}$ size) [332,398]; (ii) for a photodynamic therapy (Visudyne ${ }^{\circledR}$ (subcutaneously), containing verteporfin as active agent with verteporfin:DMPC and EPG (egg yolk phosphatidylglycerol) (1:8, drug total lipid molar ratio), 150-300 nm size) [332]; or (iii) as local anesthetic depot form formulation with extended release properties (Expare ${ }^{\circledR}$, containing bupivacaine as active agent, with a mixture of DEPC (1,2-dierucoyl-sn-glycero-3-phosphocholine, C22:1):DPPG:cholesterol and tricaprylin (=1,2,3-trioctanoylglycerol)), 24-31 $\mu \mathrm{m}$ ) [332].

Another application of lipid vesicles is in vaccination, for the prevention of certain diseases via a prophylactic activation of the immune system [338-340]. One of the successfully used product was Inflexal ${ }^{\circledR} \mathrm{V}$, applied for several years intramuscularly as virosomal adjuvant influenza vaccine [338,399-401]. It consisted of $150 \mathrm{~nm}$-sized lipid vesicles (DOPC:DOPE, 75:25, molar ratio [332]) that contained reconstituted influenza virus envelopes (the antigen glycoproteins that are localized on the surface of the virus particles, the "spike proteins"), without the inner capsid proteins and without the capsid-trapped genetic information (no viral RNA). Figure 27 is a schematic representation of an influenza virosome, together with an electron microscopy image of such virosome particle [338]. The virosomes were prepared by using the detergent depletion method [402]. A current challenge for the development of seasonal influenza vaccines that are based on the use of the spike proteins is the development of production processes of the spike proteins that are not based on the cultivation of human influenza viruses in eggs, since amino acid substitutions in the spike proteins may occur in the eggs and cause an antigenic mismatch between the vaccine and the circulating virus [403].

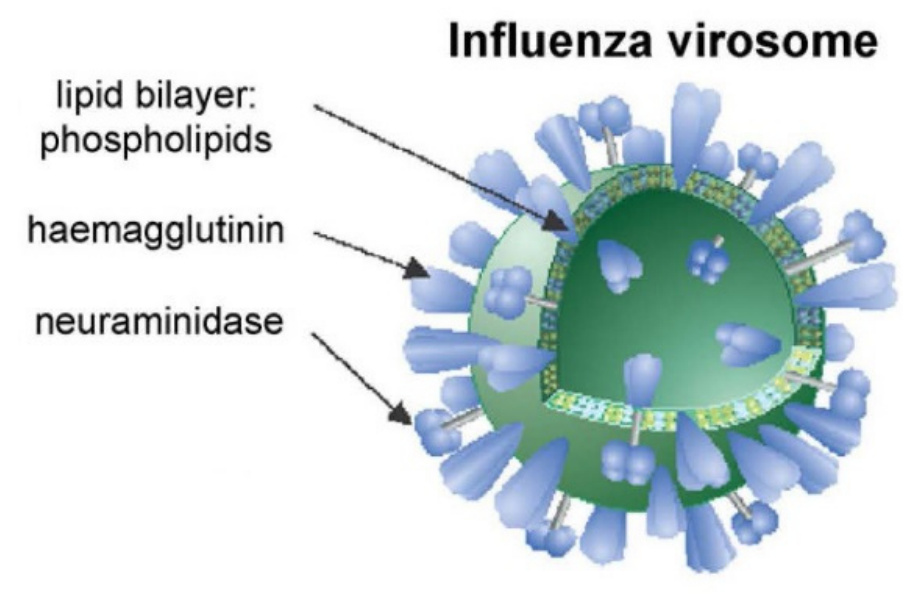

(a)

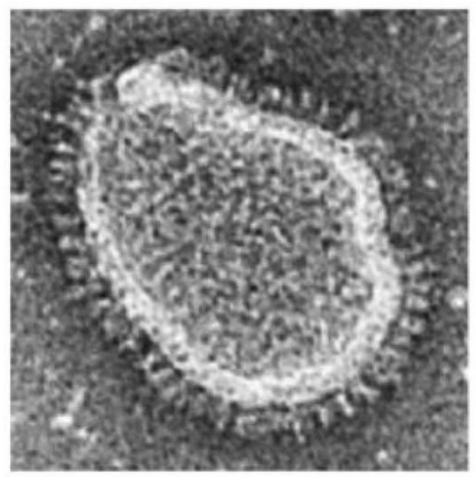

(b)

Figure 27. Application of lipid vesicles for vaccination. Schematic representation (a) and transmission electron microscopy image $(\mathbf{b})$ of an influenza virosome (diameter: $150 \mathrm{~nm}$ ) consisting of the phospholipids DOPC and DOPE (75:25, molar ratio) as membrane forming amphiphiles, with the two membrane-integrated antigene glycoproteins ("spike proteins") haemagglutinin and neuramidase, as present in the vaccine product Inflexal ${ }^{\circledR}$ V, as published by Herzog et al. (2009) [338]. This virosome preparation was made by using the "detergent-depletion method" [401,402]; see Chapter 4, Figure 17a. Reproduced with permission from [338], Elsevier, 2009.

In addition to these selected examples of the application of lipid vesicles for the treatment of diseases and as prophylactic vaccines, there are more examples of liposomal products which are in clinical use and many more that currently are in clinical trials (or were in clinical trials and failed to become commercial products) [354]. 
There is also a great potential for the use of liposomes for the treatment of noncancer diseases [404]. One example is for the treatment of rheumatoid arthritis (a systemic autoimmune inflammatory disease) [405-408]. Another example is for the treatment of hyperammonemic crises, via intraperitoneally administered liposomes for scavenging excess levels of ammonia [409,410].

Among the most recent developments of liposomes for biomedical applications, ThermoDox ${ }^{\circledR}[332,396,411-413]$ and Onpattro ${ }^{\circledR}$ (patisiran) [1] are worth mentioning.

\subsubsection{ThermoDox ${ }^{\circledR}$}

The concept of ThermoDox ${ }^{\circledR}$ is illustrated in Figure 28. This liposomal product containing entrapped doxorubicin as active agent was developed as (mild) hyperthermiainduced liposomal drug release system [414], also called "temperature-sensitive liposomes", TSL [412]. The vesicle membrane consists of DPPC, stearoyl-lyso-PC and DSPE-PEG2000, at a molar ratio of 86:10:4 $[332,396,411,412]$. The idea behind the development of ThermoDox ${ }^{\circledR}$ is the controlled and rapid release of doxorubicin as anti-tumor drug at the site of an inoperable tumor upon locally heating the tumor site [411,412] (Figure 28). The general concept of such liposomal controlled-release system was already presented in pioneering fundamental studies by Yatvin et al. (1978) [415] and Weinstein et al. (1979) [416]. Knowledge from basic studies on the main phase transition temperature of phospholipids, on the temperature-dependent permeability of phospholipid bilayers for water-soluble molecules (highest at $T_{\mathrm{m}}$ ), and on the efficient, active loading of lipid vesicles with doxorubicin (via a pH gradient [414]) was essential for the development of ThermoDox ${ }^{\circledR}$, which reached phase III clinical studies [417]. The main phase transition temperature of DPPC is $41.3 \pm 1.8^{\circ} \mathrm{C}$ [101]; see Section 2.12 and Figure 12a. Therefore, high doxorubicin release is expected to occur at $T \sim 41^{\circ} \mathrm{C}$. The presence of the "lyso-lipid" stearoyl-lyso-PC (=1-stearoyl-2-hydroxy-sn-glycero-3-phosphocholine = mono-stearoyl-phosphatidylcholine, abbreviated as MSPC [332,411,413]) in the bilayer results in a slight reduction of $T_{\mathrm{m}}$ (by $\sim{ }^{\circ} \mathrm{C}$ ), allowing the drug release at even lower temperature than in the case of pure DPPC [411,412]. More importantly, however, is (i) the additional effect the micelle-forming stearoyl-lyso-PC has on the liposomal bilayer permeability at elevated temperature through the possible formation of pores (see Figure 28), and (ii) the possible permeabilization of the tumor cells by interaction with the tumor cell membrane; in the fluid state, above $T \sim 40{ }^{\circ} \mathrm{C}$, stearoyl-lyso-PC is a micelle-forming amphiphile (with packing parameter $p \leq 1 / 3$; see Equation (1) in Section 2.3). It behaves like oleoyl-lyso-PC below $T=40^{\circ} \mathrm{C}$ (see Figure 9).

Conceptually, ThermoDox ${ }^{\circledR}$ belongs to those liposomal drug delivery systems which are designed for an efficient drug release at the site where the drug is required, and ideally only on that site (so-called "stimuli-responsive liposomes" that respond either due to an external stimulus—-by heat in the case of ThermoDox ${ }^{\circledR}$ or by light [418], for example—or due to the particular situation at the target site (pathophysiological conditions [419-421]). With respect to this second possibility, an innovative approach was developed, which is conceptually very much along the same line as in the case of ThermoDox ${ }^{\circledR}$. It is the secretory phospholipase $\mathrm{A}_{2}\left(\mathrm{sPLA}_{2}\right)$-induced destabilization of DPPC vesicles at $T=36-41^{\circ} \mathrm{C}$ for the release of anti-cancer drugs at the site of certain tumors, where the sPLA2 activity is increased due to an overexpression of sPLA 2 [422-424]. Compared to the ThermoDox ${ }^{\circledR}$ approach, a "lyso-lipid" is not present in the vesicle membrane from the beginning, but it is formed in situ due to the sPLA2-catalyzed hydrolysis of DPPC, yielding palmitoyl-lysoPC together with palmitic acid (present in part as palmitate depending on the $p H$ ). The physico-chemical effects the two micelle-forming amphiphiles have on the permeability of the liposomes and as permeabilizing agents on the tumor cells at the local temperature conditions are expected to be similar to the effect stearoyl-lyso-PC (MSPC) has in the case of ThermoDox ${ }^{\circledR}$ [332]. 


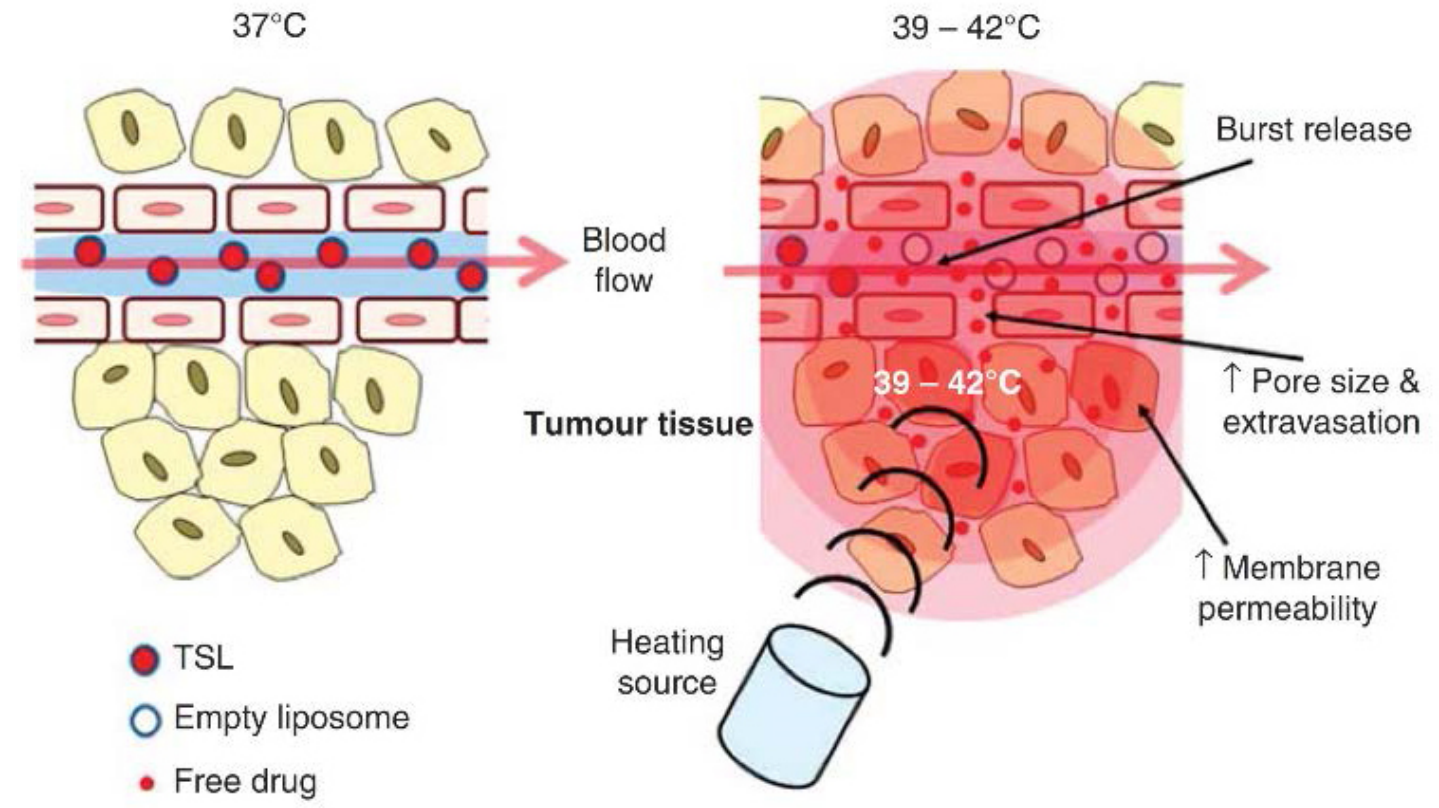

Solid Phase

Liquid Phase

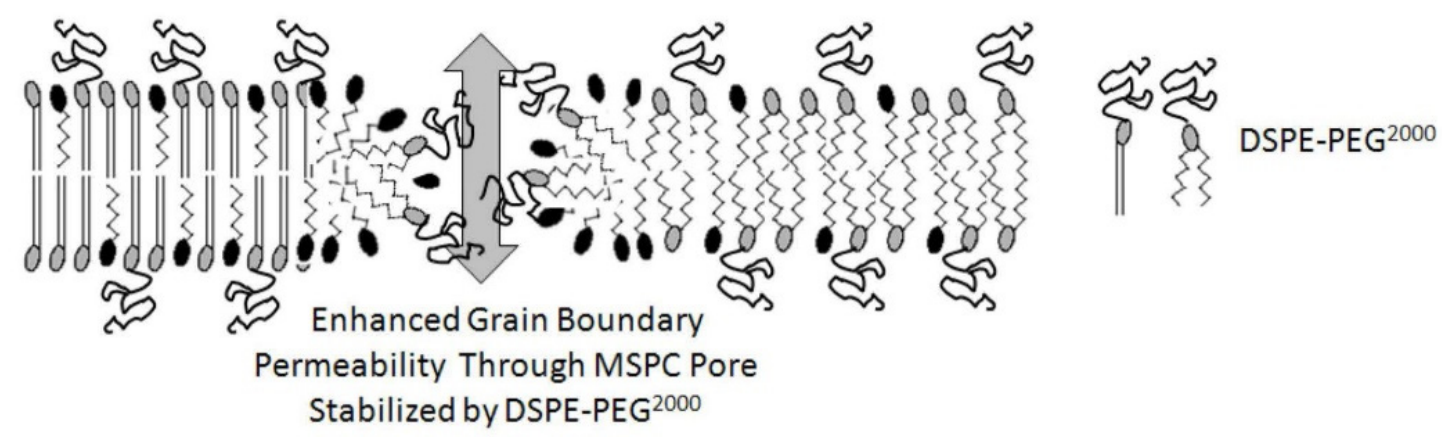

Figure 28. Application of lipid vesicles as temperature-sensitive drug delivery systems. Top: Schematic representation of the general concept for the use of temperature-sensitive liposomes (TSLs), as described by May and Li (2013) [412]. Mild hyperthermia $\left(T=39-42{ }^{\circ} \mathrm{C}\right)$ induced by a heating source at the site of the tumor can trigger the burst-release of anti-cancer drug molecules from administered TSLs. This creates high local concentrations of the drug within the tumor vasculature, promoting extravasation into the tumor. The hyperthermia also increases the permeability of the tumor cell membrane (possibly supported by the release of membrane-active components from the TSLs), which leads to increased drug uptake by the tumor cells. Bottom: Illustration of the suggested effect the lyso-lipid stearoyl-lyso-PC (=mono-stearoylphosphatidylcholine $=$ MSPC) present in the TSL ThermoDox ${ }^{\circledR}$ (together with DPPC and DSPE-PEG2000) is thought to have at elevated temperature on the liposome permeability towards the entrapped anti-cancer drug doxorubicin, as published by Landen et al. (2011) [411]. At $T \sim T_{\mathrm{m}}, \mathrm{MSPC}$ accumulates between the rigid, solid-ordered $\left(s_{\mathrm{o}}\right)$, and fluid, liquid-disordered $\left(l_{\mathrm{d}}\right)$, domains (at the "grain boundaries") and forms pores due to the micelle-forming propensity of MSPC in its fluid state. Such pore formation explains the rapid release of doxorubicin from the liposomes once $T=T_{\mathrm{m}}$ is reached. The schematic drawings of DSPE-PEG2000 illustrates the two temperature-dependent physical states of this amphiphile, "ordered" and "fluid". Reproduced from [412], copyright (C) Taylor and Francis, 2013; and with permission from [411], Bentham Open, 2011.

\subsubsection{Onpattro ${ }^{\circledR}$}

Onpattro ${ }^{\circledR}$ (patisiran), FDA-approved in 2018, was developed for the i.v. delivery of vesicle-entrapped siRNA (short interfering RNA) for the treatment of polyneuropathies resulting from the hereditary disease transthyretin-mediated amyloidosis, whereby the entrapped drug (siRNA) acts by inhibiting the synthesis of the transthyretin protein in the liver (hepatocytes as target cells), as explained by Akinc et al. (2019) [1] in their "Onpattro ${ }^{\circledR}$ 
story". This "story" is an excellent, important and exciting example of how a commercial product - and more than that, a useful and versatile platform for the delivery of various types of RNA or plasmid DNA [3,425-427] - was successfully developed on the basis of previous fundamental studies dealing with, for example, (i) the aggregation behavior of amphiphilic lipids in aqueous media, i.e., the influence of the lipid's molecular shape on the type of aggregates formed, and (ii) the reproducible preparation of submicrometer-sized vesicles with a simple procedure (related to the "ethanol-injection method", whereby rapid mixing turned out to be essential [3,428,429]).

The role of the lipidic delivery system is to protect the polyanionic siRNA molecules by entrapping them in the interior of the vesicles, and to allow for the release of the trapped siRNA molecules in active form inside the hepatocytes, once the "vesicles" (which actually turned out to be lipid nanoparticles; see Figure 29) are taken up by these cells via endocytosis (mediated by apolipoprotein $\mathrm{E}$ which binds to the surface of the lipid nanoparticles and to the hepatocytes' apolipoprotein E receptor [1,425]). These requirements could be fulfilled by (i) using the "helper lipids" DSPC and cholesterol, (ii) a "diffusible" PEGylated amphiphile (for controlling the size of the lipid nanoparticles and for providing steric stabilization to prevent aggregation during lipid nanoparticle formation and to increase the stability in the biological milieu; see Chapter 3), (iii) an appropriate cationic ionizable amphiphile (which not only forms a complex with siRNA during lipid nanoparticle formation but is also responsible for the release of siRNA from the endosomes into the cytoplasm after endocytosis), and (iv) a suitable preparation method [425,429-433]; see Figures 29 and 30.

A

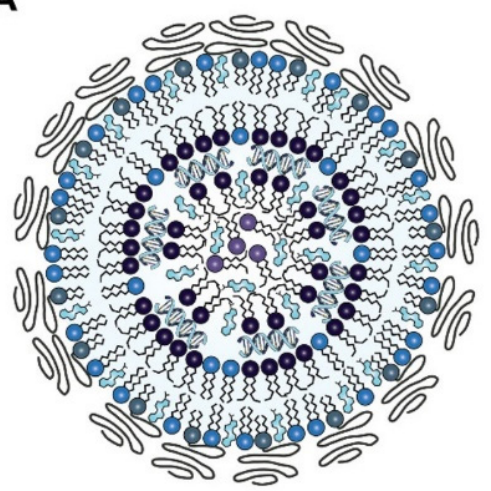

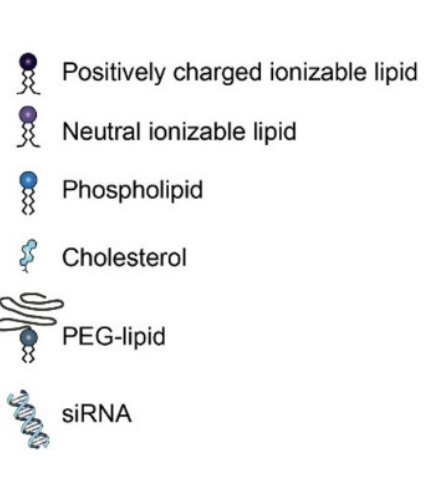

B
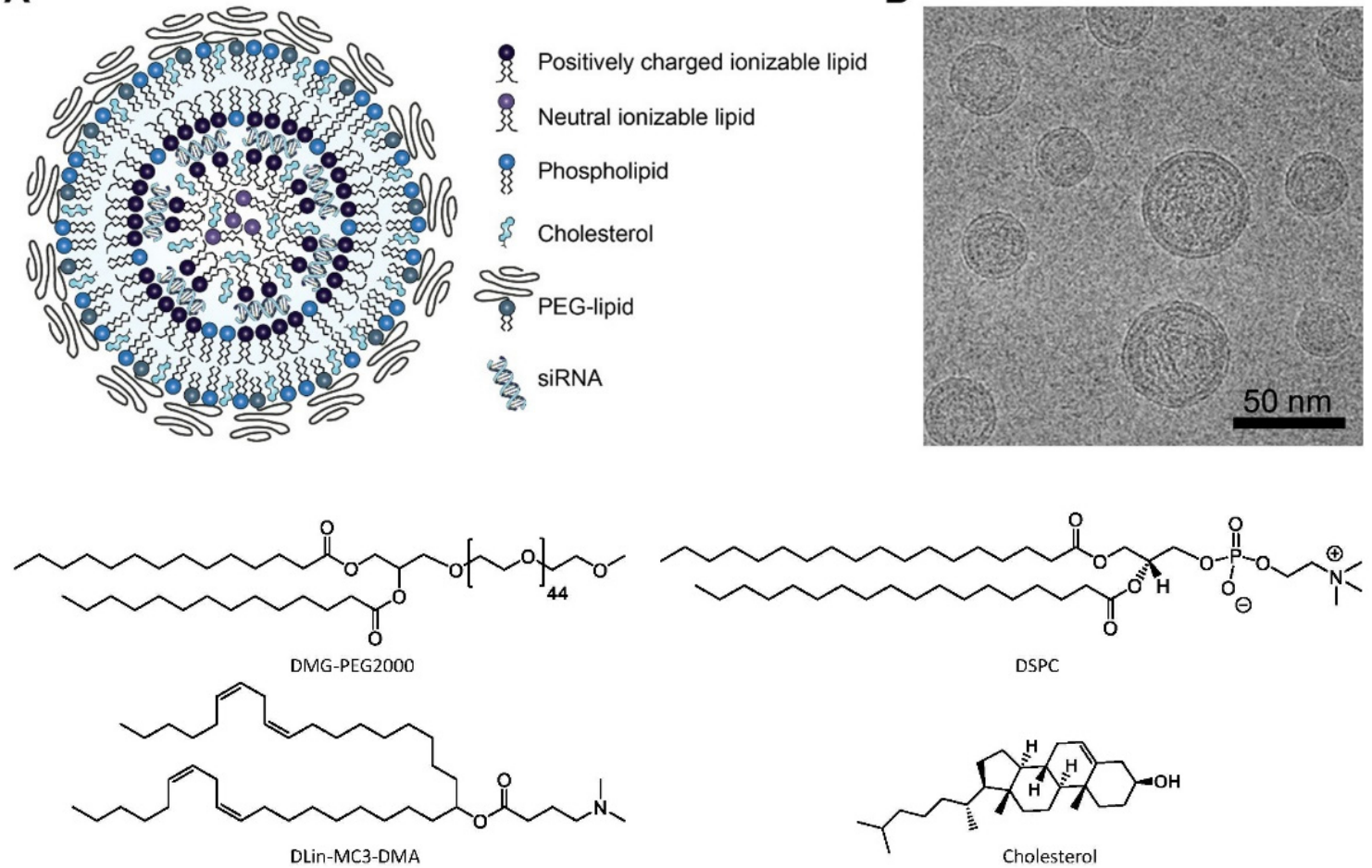

Figure 29. Morphology of the aggregates and chemical structures of the amphiphilic lipids present in Onpattro ${ }^{\circledR}$, an application of lipid nanoparticles (LNPs) for the delivery of siRNA to hepatocytes. Top: (A) Schematic representation of a cross-section through a spherical LNP containing entrapped siRNA, as published by Kulkarni et al. (2019) [429]. (B) Cryo-TEM image of a dispersion of siRNA-containing LNPs (bar: $50 \mathrm{~nm}$ ) [429,430]. Bottom: Chemical structures of the lipids constituting the siRNA-containing LNPs, the phospholipid DSPC, cholesterol, the PEGylated lipid DMG-PEG2000 and the ionizable cationic lipid DLin-MC3-DMA, used at a molar ratio of 10:38.5:1.5:50 [429]. Reproduced with permission from [429], American Chemical Society, 2019.

Since cationic lipids are known to be toxic $[434,435]$, the key challenge was to design and synthesize an amphiphile that (i) is able to act as cationic lipid during the lipid nanopar- 
ticle preparation and to promote siRNA release from the acidic endosomes, and (ii) has low toxicity in neutral form when in contact with cells. To tackle this challenge, detailed basic studies were necessary for finding an optimal "ionizable" cationic amphiphile which fulfilled the requirements of serving as siRNA complexing lipid and the same time being of low toxicity. After synthesizing more than 300 ionizable lipids, an optimal compound was found $[1,425]$. This amphiphile, dilinoleylmethyl-4-dimethylaminobutyrate, abbreviated as DLin-MC3-DMA (Figure 29) has a tertiary amine in the polar head group, with a $\mathrm{p} K_{\mathrm{a}} \sim 6.4$ of the protonated form (difficult to determine experimentally [427]). DLin-MCS-DMA is positively charged at the acidic conditions used during the initial stage of the nanoparticle preparation (using an ethanol solution containing all lipids and an aqueous solution containing siRNA at $p H=4.0$ ), and becomes neutral, non-toxic, after the $p H$ is increased to $p H=7.4$ and ethanol is removed by dialysis [430,431,433]; see Figure 30. Once taken up by the liver cells through endocytosis, the escape of siRNA from the endosomes is promoted by interactions between positively charged DLin-MC3-DMA molecules, which interact with negatively charged endogenous lipids, resulting in endosome-disrupting, inverted, non-bilayer forming structures (pair of cationic and anionic lipids that have the propensity to form inverted micelles, with packing parameter $p>1$ ) [425].

Overall, this work by Cullis' team and collaborators is not only remarkable and fascinating, but it is also a nice example for teaching, since it illustrates (i) how important fundamental studies are, (ii) how important it is to consider details of the chemical structure of the key molecules involved, (iii) how important the engineering component is for the preparation of aggregates that are only kinetically stable and not thermodynamically, and (iv) how important a careful analysis of the samples prepared is, so that further improvements and applications to related delivery challenges are possible [436]. With respect to this latter point, by using cryo-TEM it became clear that the roughly $100 \mathrm{~nm}$-sized aggregates that are obtained by the procedure used are not conventional lipid vesicles (liposomes) with an entirely aqueous interior in which the entrapped molecules are molecularly dissolved or present as crystalline precipitates (Figure 25). The lipidic siRNA-containing aggregates contain a substantial amount of lipids in the interior as well, the ionizable lipid as stabilizing complex with siRNA, as inverted micelles [425]. For this reason, spherical siRNA-containing lipid aggregates are called lipid nanoparticles (LNPs) and not lipid vesicles or liposomes. However, the term "lipid nanoparticle" is frequently also used as general term to include any type of lipid aggregates with sizes in the sub-micrometer range [1,425]. Therefore, lipid vesicles (liposomes) may also be called LNPs, it is just a matter of how LNPs are defined. For this review article, however, we distinguish the type of $100 \mathrm{~nm}$-sized lipidic RNA particles from conventional unilamellar vesicles of similar size (LUVs) by only calling the type of lipidic siRNA particles as LNPs.

The suggested mechanism for the formation of siRNA-LNPs upon mixing an ethanolic solution containing the lipids and an aqueous solution containing siRNA, followed by dialysis and $p H$ value increase, is shown in Figure 30 [430,437].

\subsubsection{Lipid Nanoparticle (LNP) mRNA COVID-19 Vaccines}

Although mRNA is considerably larger than siRNA [5], the general technology used for the preparation of siRNA-LNPs is conceptually the same as the one for the preparation of mRNA-LNPs [425-427,438-440], whereby the structure of the LNPs might be different in the two cases [5,441-443]. This novel type of mRNA-LNPs is investigated for potential use in cancer immunotherapy [444], and it was developed successfully as one of the novel vaccine types against the COVID-19 virus, which spread all over the world and caused the corona virus (COVID-19) pandemic $[3,4]$. The ingredients of the two mRNA vaccines that were developed in 2020 by Pfizer/BioNTech and Moderna and approved worldwide as first mRNA vaccines are compared in Figure 31 [3,5]. 

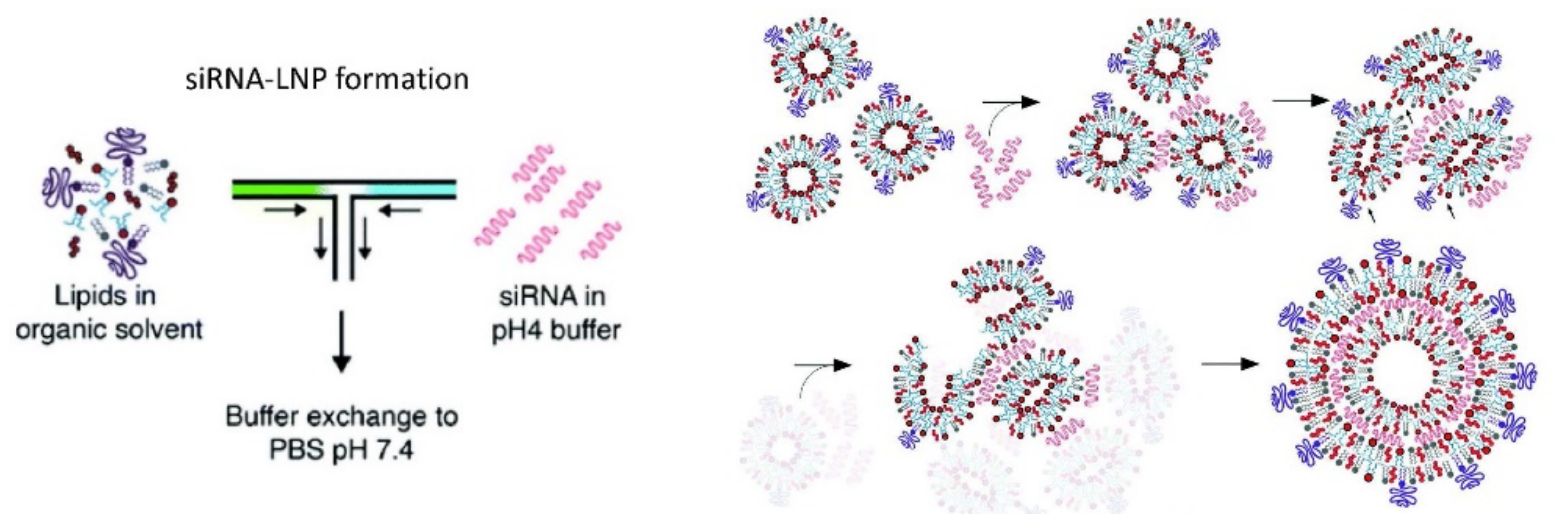

\section{凡 Positively charged ionizable lipid 8. Neutral ionizable lipid

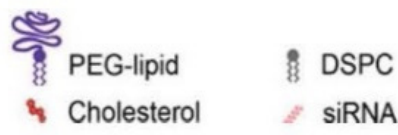

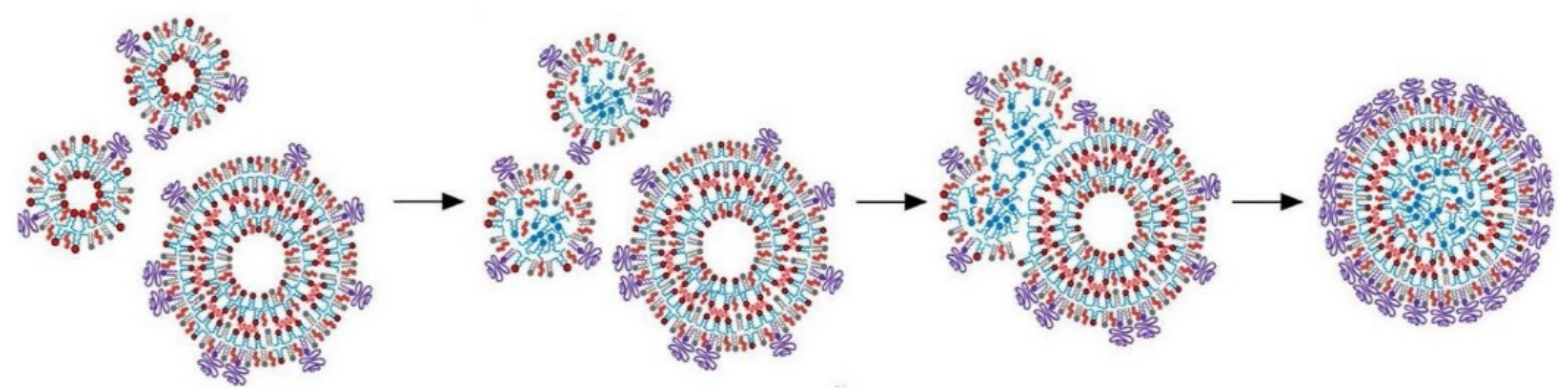

$\mathrm{pH} 4.0$

$\mathrm{pH} 7.4$

Figure 30. Schematic representation of the formation of siRNA-lipid nanoparticles (LNPs) for the delivery of siRNA to hepatocytes. Top, left: Drawing of the rapid mixing of an ethanolic solution containing the lipids in appropriate concentration and ratio (lipids in water-miscible organic solvent) with an aqueous solution of siRNA in buffer solution at $p H=4.0$, using a T-mixer, followed by dialysis (buffer exchange to PBS at $p H=7.4$ ), from Kulkarni et al. (2020) [437]. Top, right: Illustration of the events taking place at the first phase of the LNP formation, after mixing at $p H=4.0$, as published by Kulkarni et al. (2019) [437]. Experiments demonstrate that positively charged lipid vesicles form at the beginning, before siRNA molecules interact. The positively charged vesicles get then coated by anionic siRNA molecules, which leads to vesicle aggregation and the formation of multilamellar structures, which then undergo further transformations as shown in drawings at the bottom. Bottom: Proposed mechanism for the formation of siRNA-containing LNPs in the second phase of the preparation process, as published by Kulkarni et al. (2018) [430]. Upon neutralization by dialysis with PBS $(p H=7.4)$ of the cationic ionizable lipids present in the dispersion of multilamellar structures, which form in the first phase at $p H=4.0$, the cationic ionizable lipids get deprotonated and generate a metastable oil-phase, which transforms via fusion processes to the final LNPs with surface localized PEG chains and internal siRNA molecules that are sandwiched between layers of lipids and a lipid core consisting of neutralized lipids [430,437]. Reproduced with permission from [437], Royal Society of Chemistry, 2020; and from [430], American Chemical Society, 2018.

Compared to the lipids of the siRNA-LNPs of Onpattro ${ }^{\circledR}$ (Figure 29), there are differences in the chemical structures of the cationic ionizable lipids and the PEGylated lipids in the two mentioned mRNA COVID-19 vaccines (Figure 31), while the "helper lipids" are the same, DSPC and cholesterol. Cryo-TEM images of mRNA-LNPs show that they may have a non-spherical structure, with a non-homogeneous distribution of mRNA within the particles, depending on the conditions $[5,427,445]$, different from Onpattro ${ }^{\circledR}$ siRNA-LNPs; see Figure 29. What seems to be evident is that the interior of the mRNA particles contains 
the ionizable cationic lipid that is present in large quantities, and water, forming inverted micelle-like structures, as illustrated in Figure 31.
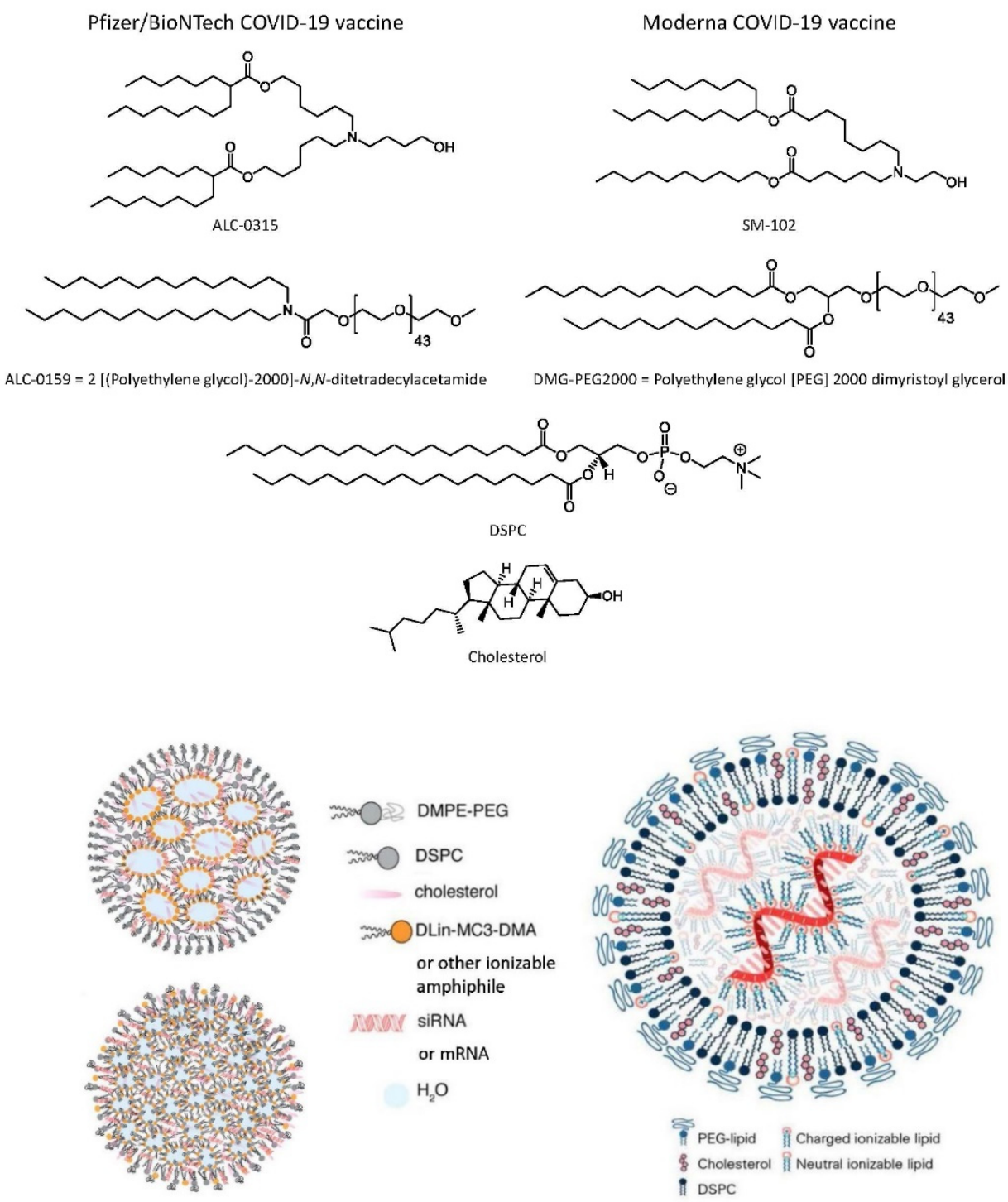

Figure 31. Novel application of lipid nanoparticles (LNPs) for the delivery of mRNA for vaccination. Top: Chemical structures of the lipids present in the first two FDA-approved COVID-19 mRNALNP vaccines that were developed by Pfizer/BioNTech (left) and Moderna (right) used in 2021 for vaccination; see Buschmann et al. (2021) [3]. Bottom, left: Schematic representation of two of the models that were proposed for the structure of siRNA-LNPs or mRNA-LNPs, either with a "nanostructured core" [442,443] (top), or with a "homogenous core" (bottom). The drawings illustrate the possible situations in the case of siRNA-LNPs, modified drawing published in [441]. The "homogenous core model" got support from dynamic nuclear polarization-enhanced NMR spectroscopy; see Viger-Gravel et al. (2018) [441], and see also [5]. In both cases, the LNPs are assumed to be spherical with the ionizable cationic amphiphiles being localized mainly inside the particles, together with a substantial amount of aqueous solution, forming inverted internal structures to which mRNA molecules are associated. Bottom, right: Drawing of a mRNA-LNP, as published by Buschmann et al. (2021) [3]. Each mRNA-LNP contains 1-10 mRNA molecules [3]. Reproduced with permission from [441], American Chemical Society, 2018; and from [3], MDPI AG, 2021.

The mRNA LNP vaccines are administered intramuscularly as in the case of the virosomes mentioned above (see Figure 27). The key difference in terms of immunization between administered mRNA-LNPs and virosomes is that in the case of the virosomes 
antibodies are directly formed against the delivered antigen proteins localized on the surface of the virosomes, while for mRNA-LNPs, the spike protein antigens need to be synthesized first within the cells into which the mRNA-LNPs enter, at the cells' ribosomeson the basis of the RNA nucleotide sequence of the mRNA that is released from the LNPs into the cytosol-before the antibodies against the synthesized spike proteins are formed $[440,446,447]$.

\subsubsection{Lipid Vesicles for Transdermal or Oral Drug Delivery Applications}

Another intensively investigated route for the delivery of drugs is across the skin, so-called transdermal drug delivery [448-451]. Although progress has been made over the years, mainly by making the lipid vesicles flexible ("deformable") by using mixtures of PCs and micelle-forming amphiphiles (e.g., lyso-PC [450] or bile salts [27,448-450]), it is in general difficult to overcome the barrier provided by the outermost horny layer of the skin, the stratum corneum. The general concepts behind these investigations were elaborated by fundamental studies on the phase behavior of amphiphiles, as mentioned in Chapter 2. Some of the transdermal and topical drug delivery systems that were developed are known as Transfersomes ${ }^{\circledR}$, invasomes, and ethosomes [450,452].

A final example for biomedical applications of liposomal systems highlighted in this review article is the one illustrated in Figure 32, as published by Uhl et al. (2021) [453]. It is about the potential use of specifically designed liposomes as carrier system for oral drug delivery. As drug, the glycopeptide antibiotic vancomycin was used. The challenge for the preparation of liposomal oral drug delivery systems is (i) to overcome the chemical and colloidal instability of conventional lipid vesicles in the stomach (acidic milieu, $p H \sim 1.0-2.0$, presence of acid proteinases) and in the small intestine (slightly alkaline milieu, presence of alkaline proteinases and micelle-forming bile salts), (ii) to succeed in high drug loadings inside the vesicles; and (iii) to achieve efficient drug permeation across the mucosal layer once the vesicles reach the small intestine. In the work of Uhl et al. (2021) [453], these three requirements could be fulfilled (i) by using in addition to the main lipids egg PC and cholesterol bipolar tetraether lipids isolated from the thermoacidophile archaeon sulfolobus acidocaldarius (containing ether bonds which are chemically more stable than the ester bonds that are present in PCs, and bearing two polar head groups of variable chemical structure) [454,455]; (ii) by using an additional phospholipid carrying a cell penetrating peptide (CPP) covalently linked to the lipid's polar head group; and (iii) by applying the "dual asymmetric centrifugation method" for efficient drug loading (a centrifugal homogenization of a concentrated lipid dispersion containing the drug, followed by dilution with an aqueous solution) [456,457]; see Figure 32. The CPP had a cyclic structure (consisting of nine arginines and one lysine, R9K) to provide a higher stability against proteinasecatalyzed hydrolysis, as compared to a linear CPP. The results obtained from preclinical in vivo studies are promising (high therapeutic efficacy) [453]. In addition to this specific example, it may well be that the number of promising lipid-based formulations for oral drug delivery will increase in the future [458], not only for the delivery of antibiotics [459].

\subsection{Bioanalytical Applications of Lipid Vesicles (Bioassays and Biosensors)}

In addition to the use of synthetic polydiacetylene-based vesicles as colorimetric and fluorimetric sensor systems towards various chemical, biological and physical stimuli [390-393], there is a large number of studies on the application of lipid vesicles from naturally occurring amphiphiles in biosensor devices and for bioassays [460-467]. This type of analytical application is based on three fundamental properties of lipid vesicles: (i) the possibilities of external vesicle surface functionalization (Figure 22) [463,465], (ii) the possibilities of entrapping water-soluble molecules in the aqueous interior of the vesicles, and (iii) the possibilities of immobilizing the vesicles on a solid surface, either via covalent bonds $[468,469]$ or noncovalently, for example through the biotin-avidin (or streptavidin) system [470-472]. 

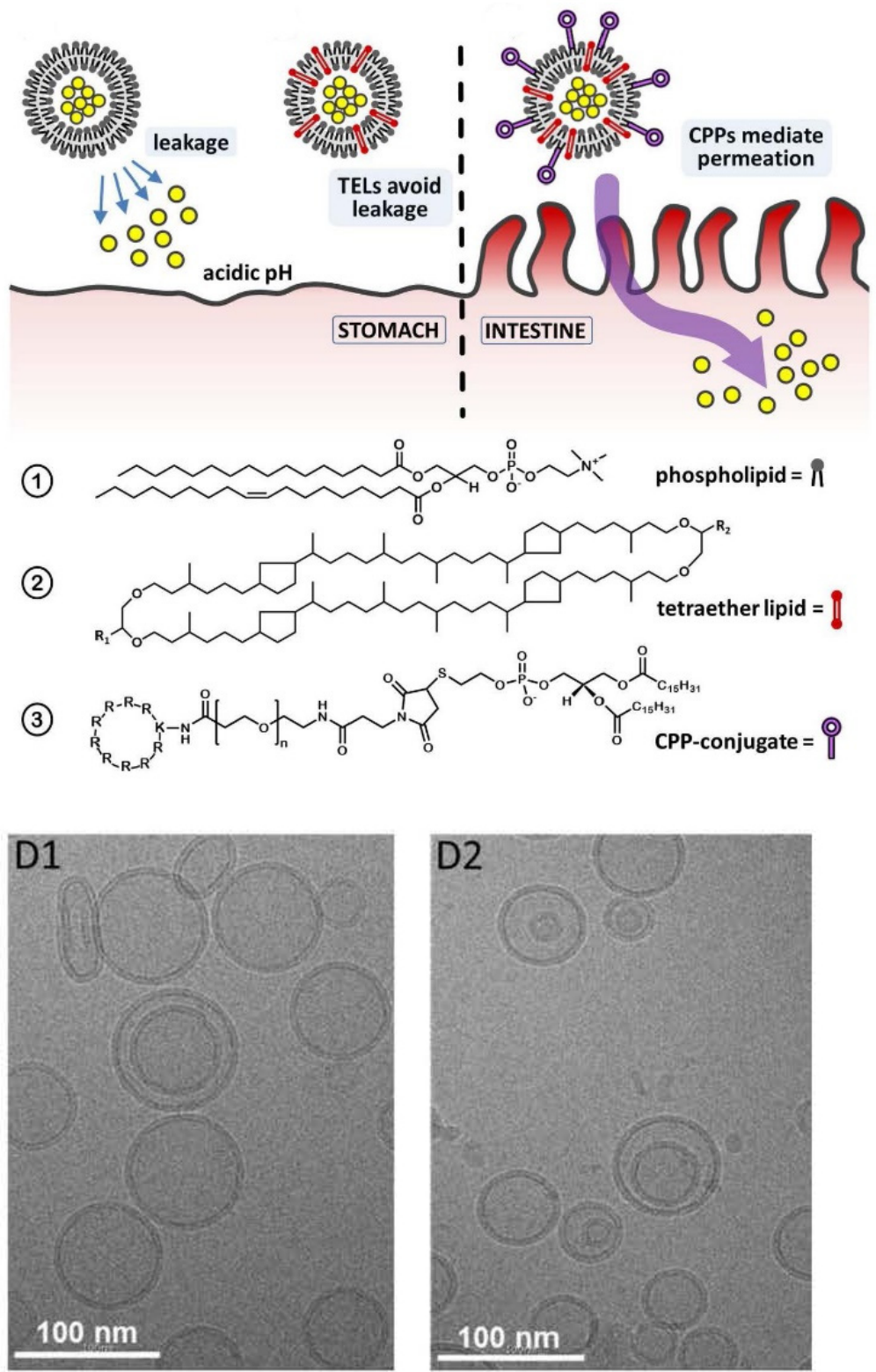

Figure 32. Application of lipid vesicles for oral drug delivery. Top: Illustration of the challenges and concept of the work published by Uhl et al. (2021) [453], for the oral delivery of vancomycin (filled yellow circles) entrapped inside lipid vesicles. Top left: Limitations of the use of conventional liposomes due to chemical instability at the acidic conditions of the stomach, which results in undesired drug release. Top, center: Increased vesicle membrane stability in the presence of bipolar tetraether lipids (TELs). Top right: Increased permeation across the mucosal layer of the small intestine due to the use of lipids carrying a cyclic cell penetrating peptide (CPP) in the polar head group. Middle: Chemical structures of the lipids used in addition to cholesterol. The phospholipid shown is POPC, a representative for the mixture of egg yolk PC (1) that was used [453]. The TELs are mixtures of glycerylcaldityltetraether lipids (2), isolated from Sulfolobus acidocaldarius. For the polar head group R, see for example Chong et al. (2012) [454]. The lipidic CPP-conjugate is a head groupmodified double chain phospholipid with two palmitoyl chains (3). The following molar ratio was used: phospholipid (1): cholesterol:bipolar tetraether lipid (2): CPP-conjugate (3) = 84:10:5:1 [453]. Bottom: Cryo-TEM image of the CPP-modified vesicles (D1) and of "control vesicles" consisting of (1) and cholesterol (9:1, mol ratio) only (D2) (bar: $100 \mathrm{~nm}$ ). The vesicles were prepared by the dual asymmetric centrifugation method [456,457]. Reproduced with permission from [453], Wiley-VCH, GmbH, 2021. 
In one type of approach, vesicle surface-localized functional groups can serve as specific binding sites for analyte molecules, whereby the trapped molecules "report" this analyte binding through a detectable signal, which correlates with the analyte binding event. This concept was applied in the work reported by Sforzi et al. (2020) [466]. DPPC vesicles were used for a fast, simple, and reproducible detection of single or double stranded DNA in biological samples (serum), as demonstrated by first proof-of-concept experiments. The analyte (DNA) binding step of this bioassay is illustrated in Figure 33. The principles of the DNA quantification on the basis of DPPC vesicles (Figure 33, left) and streptavidin-coated magnetic Sepharose beads (Figure 33, right) is the following. The DNA fragment to be quantified (the analyte) is the "TARGET" fragment. For the detection of this TARGET fragment, two conjugates need to be synthesized. The first one is a conjugate between biotin and an oligonucleotide fragment (the "ANCHOR"), which is complementary to the sequence at the $3^{\prime}$ end of the TARGET fragment ("BiotinTEG"). Incubation of the biotin-ANCHOR conjugate with streptavidin-coated beads leads to a binding of the biotin-ANCHOR conjugate to the beads. Further incubation with the TARGET fragment results in binding of the TARGET fragment to the surface of the beads while the sequence at the $5^{\prime}$ end remains free for binding to DPPC vesicles containing entrapped 5(6)-carboxyfluorescein (CF) and a surface-localized oligonucleotide sequence (the "PROBE"), which is complementary to the free sequence at the 5 ' end of the TARGET fragment. The PROBE is conjugated to cholesterol, the second conjugate to be prepared for this assay system, to keep the PROBE on the surface of the DPPC vesicles through an insertion of the cholesteryl moiety into the DPPC bilayer [473].

Experimentally, pure DPPC LUVs were first prepared by using the polycarbonate membrane extrusion method (see Chapter 4, extruded at $T=56^{\circ} \mathrm{C}>T_{\mathrm{m}}$ (DPPC) $=41.3 \pm 1.8^{\circ} \mathrm{C}$ [101], with $200 \mathrm{~nm}$ membranes for the final extrusions) in the presence of $50 \mathrm{mM}$ 5(6)-carboxyfluorescein (CF) in $3.8 \mathrm{mM}$ HEPES buffer, $0.15 \mathrm{M} \mathrm{NaCl}, p H=7.4$ [466]. At this high concentration, the fluorescence of CF is quenched due to dimer formation [474,475]. After separation of the non-entrapped CF molecules from the extruded vesicles by extensive dialysis of the aqueous vesicle dispersion against isotonic $\mathrm{HEPES} / \mathrm{NaCl}$ solution (to avoid leakage of CF from the vesicles, which may occur in osmotically unbalanced systems), the vesicles had a diameter of $110 \pm 31 \mathrm{~nm}$ (as determined by dynamic light scattering measurements) and were stable for at least 4 months if stored at $T=4{ }^{\circ} \mathrm{C}$ (in the $s_{\mathrm{o}}$ state) [466]. The cholesteryl-PROBE conjugate was added to the DPPC LUVs for spontaneous insertion of the cholesteryl moiety at $T=4{ }^{\circ} \mathrm{C}$ [466], followed by removal of non-associated conjugate molecules by dialysis. After mixing an aqueous dispersion of the magnetic Sepharose beads with surface-bound TARGET fragments with an aqueous dispersion of DPPC LUVs containing the PROBE on their surface, binding of the vesicles to the beads occurred. Upon washing with buffer solution to remove excess vesicles, the amount of vesicles bound to the magnetic beads was quantified by determining the amount of CF bound to the beads, which is expected to be proportional to the amount of DNA in the sample. Quantification of the amount of CF was done by adding Triton X-100 which caused CF-leakage from the vesicles and resulted in an increase in fluorescence $\left(\lambda_{\mathrm{ex}}=492 \mathrm{~nm}, \lambda_{\mathrm{em}}=517 \mathrm{~nm}\right)$ due to CF dilution caused by CF diffusion from the vesicles into the bulk solution. Such CF leakage occurs upon addition of the micelle-forming amphiphile Triton X-100, due to a transformation of the DPPC:cholesteryl-PROBE vesicles into mixed vesicles and finally mixed micelles, depending on the amount and concentration of added Triton X-100 [148,476-478].

With this liposomal assay, a TAREGET DNA fragment as analyte could be detected at a concentration of $7 \times 10^{-11} \mathrm{M}$ in HEPES buffer and $0.8 \times 10^{-8} \mathrm{M}$ in serum. Although this latter concentration is higher than the desired detection limit for analyzing free serum DNA associated to cancer diseases, an optimization at the level of the vesicles used might still be possible and could result in a more sensitive assay than the one developed so far [466]. 

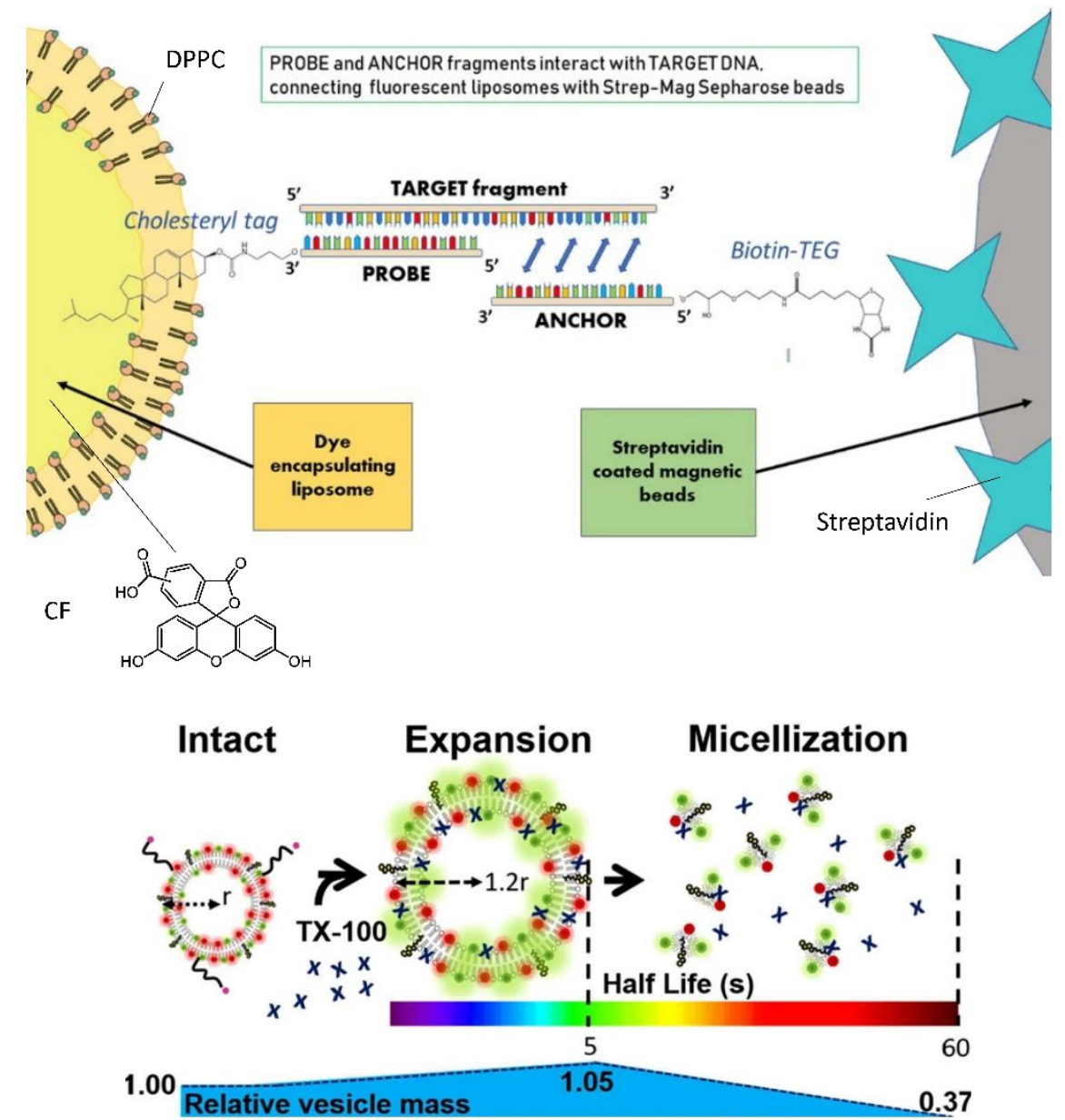

Figure 33. Application of dye-containing lipid vesicles in a bioassay for the quantitative determination of DNA, as published by Sforzi et al. (2020) [466]. Top: The first part of the assay of an oligonucleotide TARGET fragment as analyte is shown. This first part is the interaction between (i) DPPC LUVs containing encapsulated 5(6)-carboxyfluorescein (CF) at a self-quenching concentration and a membrane-embedded cholesteryl-PROBE conjugate, the PROBE being an oligonucleotide with a sequence that is complementary to the oligonucleotide sequence at the $5^{\prime}$ end of the TARGET fragment (left hand side) and (ii) streptavidin-coated magnetic Sepharose beads with a surface bound biotin-ANCHOR conjugate, the ANCHOR being an oligonucleotide with a sequence that is complementary to the oligonucleotide sequence at the $3^{\prime}$ end of the TARGET fragment (right hand side). The TARGET fragment acts as linker unit for the binding of the CF-loaded DPPC LUVs to the magnetic beads. Bottom: The second part of the assay is the release and quantification of CF from the beads-bound DPPC LUVs by adding the micelle-forming surfactant Triton X-100, as illustrated by Dalgarno et al. (2020) [478] for dispersed vesicles. "Expansion" means mixed lipid-detergent vesicles that increase in mass and size after addition of Triton X-100 before micellization starts; see [478] for details. Reproduced with permission from [466], American Chemical Society, 2020; and from [478], Springer Nature, 2019.

Another possible bioanalytical application of liposomes is their use as enzyme-containing amplification system in immunoassays [479], conceptually related to the liposomal quantification system just described. A selected example is the one described by Lin et al. (2017) [480]. In that work, liposomes containing entrapped horseradish peroxidase (HRP) were used as colorimetric amplification system in an immunoassay for the quantification of influenza virus H5N1. The concept of the work is shown in Figure 34 and explained in the following. Lipid vesicles consisting of egg PC, cholesterol, and DSPE-PEG2000-biotin (at an initial molar ratio of 70:28:2) were prepared by first dissolving the three lipids in ethanol. A volume of $0.25 \mathrm{~mL}$ of this ethanol solution was then added to $10 \mathrm{~mL}$ of an 
aqueous phosphate buffer solution $(p H=7.4)$ containing HRP. During the mixing of these two solutions, vesicle formation occurred with some of the biotin moieties being localized on the outer vesicle surface, and some of the HRP molecules becoming entrapped inside the vesicles (ethanol injection method; see Figure 17a). The obtained vesicle dispersion was sonicated using a probe-type sonicator (for vesicle size reduction and homogenization), followed by removal of non-entrapped HRP by size exclusion chromatography (using Sephadex G-100). It seems that the sonication conditions used did not lead to a substantial HRP inactivation. The size of the obtained HRP-containing lipid vesicles with surface bound biotin was $\sim 100 \mathrm{~nm}$ [480]. The vesicle dispersion was stored at $T=4{ }^{\circ} \mathrm{C}$ until use in the way shown in Figure 34.

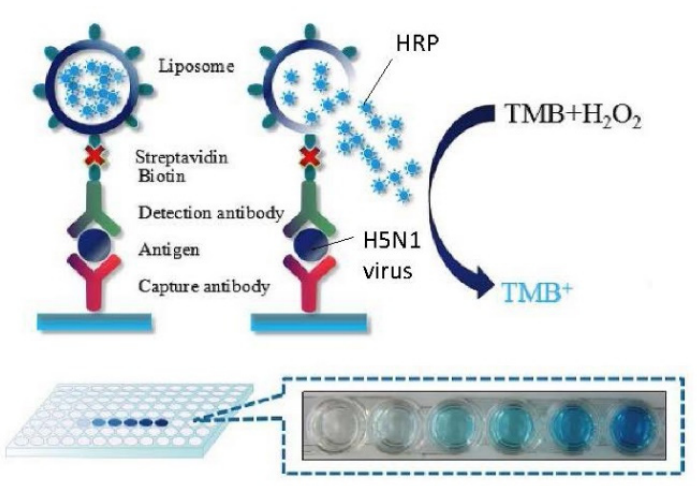

(a)

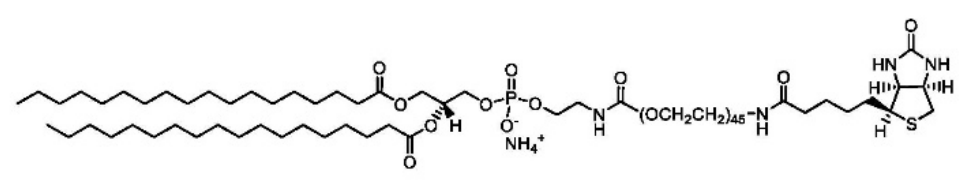

DSPE-PEG2000-biotin

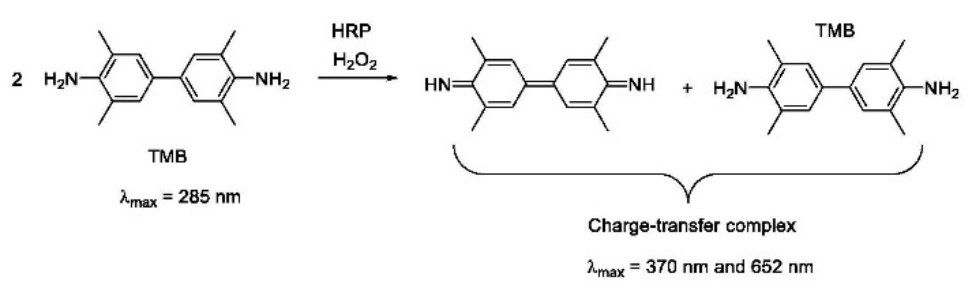

(b)

Figure 34. Application of enzyme-containing lipid vesicles in an immunoassay for the quantitative determination of influenza virus H5N1, as published by Lin et al. (2017) [480]. (a) Illustration of the concept of the method. A capture antibody is adsorbed onto the solid surface of a 96-well microplate. The antigens present in the analyte mediumH5N1 virus particles-bind selectively to the surface-bound capture antibody. Addition of a biotinylated detection antibody results in binding of the biotinylated capture antibody to the antigen. Following addition of streptavidin, added biotinylated lipid vesicles containing entrapped horseradish peroxidase (HRP) bind to streptavidin. After washing, quantification of HRP is achieved by using 3,3',5,5'-tetramethylbenzidine (TMB) and hydrogen peroxide $\left(\mathrm{H}_{2} \mathrm{O}_{2}\right)$ as substrates at $p H \sim 4-5$, whereby HRP may leak from the vesicles. (b) The one-electron oxidation of two TMB molecules-followed by a disproportionation reaction-yields a blue colored charge-transfer complex between the diimine product and TMB, with $\lambda_{\max } \sim 650 \mathrm{~nm}$; see [481]. The liposomes were prepared from egg PC, cholesterol and DSPE-PEG2000-biotin; see [480] for details. Reproduced with permission from [480], Elsevier, 2017.

A 96-well microplate was coated with H5N1 antibodies (so-called "capture antibodies") via simple adsorption. After washing steps, a dispersion of H5N1 virus particles (the antigens) was added and incubated to allow for binding of the virus particles to the capture antibodies. After a washing step, a solution of biotinylated H5N1 antibodies (so-called "detection antibodies") was added. After incubation and a washing step, a solution of streptavidin was added so that the biotin molecules covalently linked to the capture antibody could bind to one of the four binding sites of streptavidin. After a further washing step, an aqueous dispersion of the lipid vesicles with surface-bound biotin and entrapped HRP was added, followed by incubation to allow binding between the biotinylated vesicles and streptavidin. After a final washing step to remove unbound vesicles, the amount of remaining HRP was determined by using TMB $\left(3,3^{\prime}, 5,5^{\prime}\right.$-tetramethylbenzidine) and hydrogen peroxide $\left(\mathrm{H}_{2} \mathrm{O}_{2}\right)$ as HRP substrates. For the enzymatic reaction to be successful, both, $\mathrm{H}_{2} \mathrm{O}_{2}$ and $\mathrm{TMB}$, need to reach the heme group at the active site of the enzyme. For the small and neutral $\mathrm{H}_{2} \mathrm{O}_{2}$ molecule, the vesicle bilayer is expected to be quite permeable [482]. In contrast, for $\mathrm{TMB}$, the situation in terms of lipid bilayer permeability is expected to depend on $\mathrm{pH}$ (the $\mathrm{p} K_{\mathrm{a}}$ values of the protonated amines of TMB are about 4.0 and 3.1, respectively [483]). Since there is no doubt that the HRP-catalyzed oxidation reaction took place under the assay conditions (reported blue color development, Figure 34a), it seems that either TMB 
could permeate into the vesicles once TMB was added, or the HRP molecules were released from the vesicles. This latter possibility is indicated in Figure 34a. A quantification of the oxidation of TMB was carried out by analyzing the formation of the charge-transfer complex which has a blue color, with an absorption maximum at $\lambda_{\max } \sim 650 \mathrm{~nm}$ [481]; see Figure $34 \mathrm{~b}$. The use of liposomal HRP allowed the selective detection of H5N1 virus particles at $0.1-0.4 \mathrm{ng} / \mathrm{mL}$, with a detection limit of $0.04 \mathrm{ng} / \mathrm{mL}$, much lower than in the case of a conventional enzyme-linked immunosorbent assay (ELISA), [480], where only one enzyme molecule is covalently attached to a detecting antibody. It seems that the lipid vesicles contained several HRP molecules, although this was not determined in the work carried out by Lin et al. (2017) [480].

In the final example about bioanalytical applications of lipid vesicles we would like to mention here, L-lactate was quantified in blood samples by using giant vesicle-trapped L-lactate oxidase from Aerococcus viridans (LO [484,485]), HRP, and the fluorescent HRP substrate "sulfo-cyanine 7 carboxylic acid" [486]; see Figure 35. The two enzymes (LO and HRP) and sulfo-cyanine 7 carboxylic acid $\left(\lambda_{\max }=750 \mathrm{~nm}\right.$, fluorescent, $\left.\lambda_{\text {max,em }}=773 \mathrm{~nm}\right)$ were entrapped inside lipid vesicles formed from DPPC, cholesterol, and DSPE-PEG2000 (15.3:18.4:0.3, molar ratio) at $p H=7.4$ by using a $50 \mathrm{mM}$ sodium phosphate buffer solution containing $\mathrm{NaCl}$ to yield an osmolality of $300 \mathrm{mOsm} / \mathrm{kg}$. The vesicles were obtained by spontaneous hydration of a previously prepared dried lipid film with the buffer solution containing the two enzymes and sulfo-cyanine 7 carboxylic acid, to obtain a total lipid concentration of $34 \mathrm{mM}$ and $100 \mu \mathrm{M}$ sulfo-cyanine 7 carboxylic acid, followed by a heating (to $T=56^{\circ} \mathrm{C}$ ) and vortexing protocol; see [486] for details. Non-entrapped compounds were separated from the vesicles by using size exclusion chromatography (Sepharose CL-6B and Miditrap G-25). The vesicles obtained had a unimodal size distribution with a median diameter of about 7-8 $\mu \mathrm{m}$. Bovine whole blood samples to which a defined amount of L-lactate was added were then analyzed. To optimize vesicle bilayer permeability, the $\mathrm{pH}$ value of the blood was lowered from $p H=7.4$ to 6.1 to increase the amount of protonated lactate (i.e., lactic acid, $\mathrm{p} K_{\mathrm{a}} \sim 3.9$ [487]) over lactate, and with this to increase the vesicle bilayer permeability of the analyte as compared to the $p H=7.4$ conditions; see the concept of the "remote loading of vesicles" in 5.4. After adding the blood sample to a portion of the vesicle dispersion, the lactic acid molecules could permeate from the external bulk medium to the interior of the vesicles, where the oxidation of lactate to pyruvate and hydrogen peroxide $\left(\mathrm{H}_{2} \mathrm{O}_{2}\right)$ could take place. The formed $\mathrm{H}_{2} \mathrm{O}_{2}$ oxidized HRP, which in turn oxidized sulfo-cyanine 7 carboxylic acid, which resulted in a loss of the absorption with maximum centered around $\lambda=750 \mathrm{~nm}$ and as a consequence to a concomitant loss in fluorescence. With this liposomal assay, the decrease in relative fluorescence intensity was found to be directly proportional to the lactate concentration in the blood sample. Quantification was possible between 0.2 and $5.0 \mathrm{mM}$ L-lactate (final concentration in the assay), corresponding to $1.25-20 \mathrm{mM}$ in the blood [486]. Using instead of LO other oxidases may allow the quantification of other metabolites in blood, e.g., D-glucose [486].

\subsection{Application of Lipid Vesicles in Food Processing and Nutrition}

There have been a number of studies on the use of lipid vesicles (liposomes) in various areas of food industry [488] and nutrition [489], as dietary supplements (nutraceuticals [489-493]), for promoting the processing of food (for example, in cheese production, [494,495]), or for the prevention of bacterial growth (using liposome-entrapped antimicrobial peptides [496-498]); see Figure 36a. Often, the role the liposomes should play in these applications is to protect entrapped sensitive ingredients from being degraded [490]. Another possibility is to include antioxidants (e.g., $\alpha$-tocopherol, $\beta$-carotene, vitamin C), flavor molecules, or vitamins that may not have antioxidant activities [490]. Due to the particular structure of liposomes, the co-loading of water-soluble as well as membrane-soluble molecules within the same liposomes-for example vitamin $\mathrm{C}$ and $\beta$-carotene (Figure 36b) - is possible for potential applications as food additives [499]. 

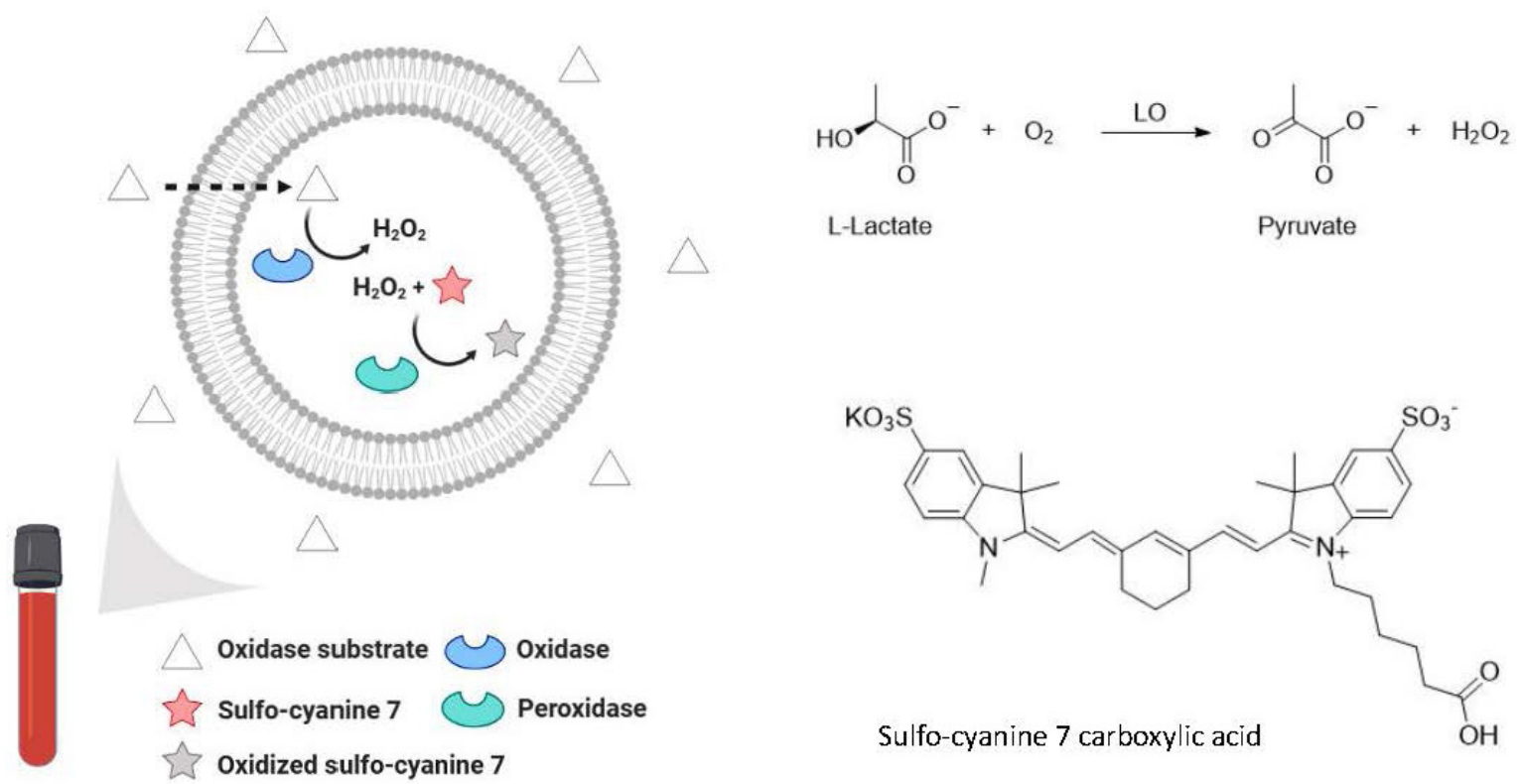

Figure 35. Application of enzyme-containing lipid vesicles for the quantification of L-lactate in whole blood samples, as developed by Matoori and Mooney (2020) [486]. Schematic representation of the principle of the method (not drawn to scale). Lipid vesicles of about 7-8 $\mu \mathrm{m}$ diameter, formed from DPPC, cholesterol, and DSPE-PEG2000, containing entrapped L-lactate oxidase from Aerococcus viridans (LO, "oxidase"), HRP ("peroxidase"), and fluorescent sulfo-cyanine 7 carboxylic acid ("sulfo-cyanine 7") were prepared at $p H=7.4$. L-lactate ("oxidase substrate") present in blood samples added to the vesicles $(p H=6.1)$ permeate across the vesicle bilayer(s) (indicated by the dashed line). Inside the vesicles L-lactate is oxidized to pyruvate and $\mathrm{H}_{2} \mathrm{O}_{2}$ (catalyzed by LO), followed by a HRP-catalyzed oxidation of sulfo-cyanine 7 carboxylic acid to "oxidized sulfo-cyanine 7", which can be monitored by a decrease in fluorescence intensity; see text and [486] for details. Drawing reproduced with permission from [486], WILEY-VCH Verlag GmbH \& Co. KGaA, 2020.

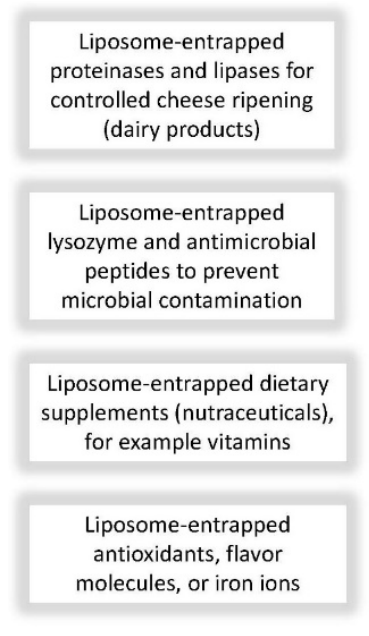

(a)

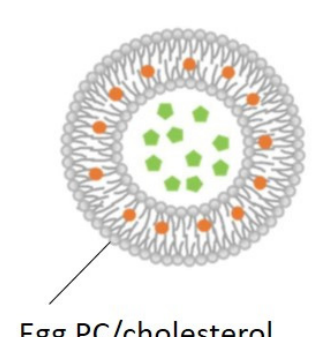

Egg PC/cholesterol
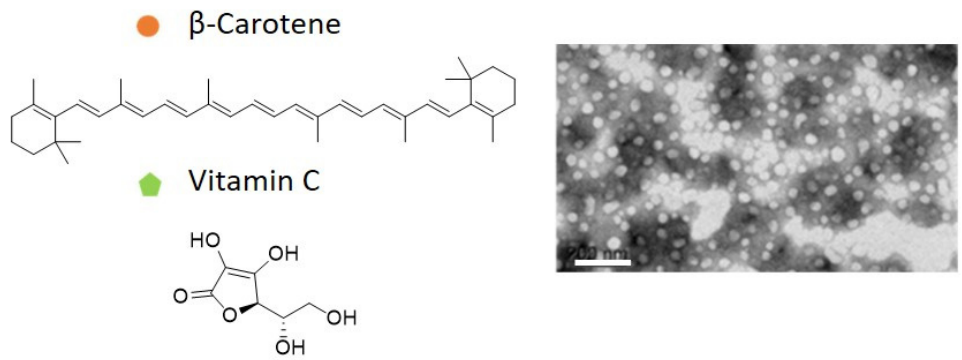

(b)

Figure 36. Application of lipid vesicles in food processing and nutrition. (a) Some of the areas of food technology and nutrition where the use of liposomes has been explored or where liposome-based products are used commercially as food supplements; see [488-490,496,500]. (b) Illustration of lipid vesicles that were co-loaded with water-soluble (vitamin C) as well as membrane-soluble molecules ( $\beta$-carotene) and of a negatively stained TEM image of the vesicles prepared (bar: $200 \mathrm{~nm}$ ), as published by Liu et al. (2020) [499]. A mixture of egg PC and cholesterol was used as vesicle membraneforming amphiphiles. Sonication was applied for lipid dispersion and vesicle size reduction [499]. The reported size of the vesicles obtained was about $250 \mathrm{~nm}$ (as determined by dynamic light scattering), larger than the objects seen in the TEM image obtained from dried samples (stained with sodium phosphotungstate). Reproduced with permission from [499], Elsevier, 2020. 
In the case of the application of liposomes for the production of cheese, in the pioneering work of Kheadr et al. (2000) [494], negatively charged phospholipid vesicles containing entrapped proteinases were added to the curd in Cheddar cheese production for controlling the development of cheese flavor and texture. With the exception of liposomal nutritional products that are (or were) commercially available [489], there is not (yet) much known about real applications of liposomal preparations in the food industry, despite the many efforts and many possibilities lipid vesicles offer [491,500-505]. This is somewhat surprising because one of the advantages of vesicles prepared with natural amphiphiles is their biocompatibility and the beneficial health effects phospholipids can have by themselves [506-509]. As discussed previously [488], reasons that may hinder real applications include (i) a lack of knowledge about the interaction of the liposomes (and possibly entrapped or membrane-embedded active ingredients) with the food components, (ii) the costs of the amphiphilic raw materials for the formation and loading of the liposomes, (iii) not yet developed simple and reproducible methods for a cheap liposome manufacturing, and (iv) lack of a deep knowledge about the fate of the liposomes in the gastrointestinal tract.

Concerning the required cheap and efficient large scale industrial production of liposomes containing desired ingredients of nutritional benefit, for example, by microfluidization [498], one recent example worth mentioning is the development of oral liposomal vitamin C, as published by Łukawski et al. (2020) [510] (Figure 37) and Prantl et al. (2020) [511]. In both studies, lipid vesicles were prepared by using a water-miscible organic solvent which is nutritionally and pharmacologically accepted, glycerol [510], or ethanol [511], whereby the phospholipid products and the general procedure applied for the preparation of the vesicles containing entrapped vitamin $C$ (as sodium ascorbate or ascorbic acid) were quite different. Therefore, it is not surprising that the characteristics of the two liposome dispersions prepared were also rather different.

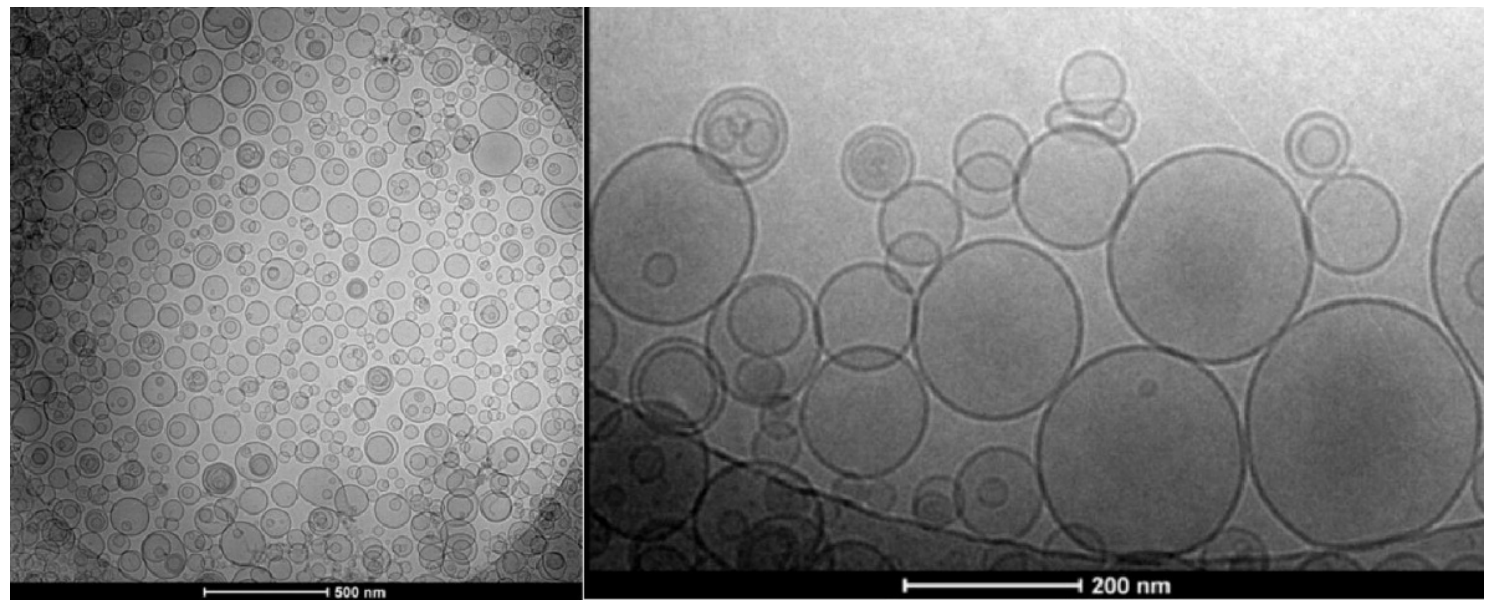

Figure 37. Applications of lipid vesicles for the delivery of food supplements. Cryo-TEM image of a soybean PC/“rape seed lecithin" vesicle dispersion containing entrapped vitamin $C$, as obtained by a simple procedure involving the use of glycerol and mechanical mixing steps, as published by Łukawski et al. (2020) [510]. Reproduced with permission from [510], Taylor \& Francis, 2020.

Łukawski et al. (2020) [510] used soybean phosphatidylcholine (Phospholipon 90 G, i.e., a mixture of unsaturated phosphatidylcholines from soybeans, with 94-100\% PC [512]) and a crude phospholipid mixture from rapeseeds containing PCs but also other phospholipids. This phospholipid mixture is called "rapeseed lecithin" by the producer because in agriculture and food technology, the term "lecithin" is used for phospholipid mixtures and not for phosphatidylcholines only; see Table 2. Aqueous phospholipid dispersions at higher than $20 \mathrm{wt} \%$ lipid were prepared to optimize the vitamin C encapsulation efficiency. The liposomes were obtained by using a glycerol solution containing the lipids (50 wt $\%$ ) 
and an aqueous solution containing vitamin C (20 wt\% vitamin C). Interestingly, and remarkably, simple mixing of these two solutions resulted in a rather homogeneous, highly viscous, gel-like vesicle dispersion. A cryo-TEM analysis of the vitamin C-containing vesicles indicated the presence of mainly unilamellar vesicles with $180 \pm 30 \mathrm{~nm}$ diameter (Figure 37), which was in good agreement with dynamic light scattering measurements $(168 \pm 25 \mathrm{~nm})$ [510]. Therefore, the simple procedure used not only yielded rather well defined vesicles, but the method also appears applicable to large volumes. Overall, this liposomal vitamin $C$ showed elevated bioavailability if compared to an aqueous vitamin $C$ solution, as demonstrated by analyzing the vitamin $C$ content in the serum of 20 volunteers after oral administration [510]. Such enhanced bioavailability in humans was also found in the work of Prantl et al. (2020) [511]. In that work, however, a powder from sunflower seeds ("sunflower lecithin") was used, ethanol as water-miscible solvent and bath sonication for dispersing the lipids and for homogenizing the vesicle dispersion to some extent. This mechanical treatment resulted in a reported size range of the vesicles between $400 \mathrm{~nm}$ and $3 \mu \mathrm{m}[511]$.

In both studies relatively crude phospholipid mixtures and simple preparation procedures were used. This appears an advantage for real applications. Nevertheless, based on the data available from literature, it seems that more fundamental studies are required, as applications of lipid vesicles in food science and technology are still under development. The beneficial health effects that some of the vesicle forming phospholipids are known to have [506-509] should make this type of application very attractive for further investigations.

\subsection{Application of Lipid Vesicles for Membrane Protein Reconstitution, for the Preparation of Synthetic Cell-like Compartments ("Artificial Cells"), and as Models of "Protocells"}

Lipid vesicles have been used for many years as biomembrane-mimicking model systems for the reconstitution and investigation of membrane proteins; see also the related application of virosomes (Figure 27). Membrane protein-containing lipid vesicles are called "proteoliposomes" [513-515]. Systematic studies on the detergent-assisted reconstitution of membrane proteins in LUVs were carried out by Rigaud, Lévy, and their collaborators [516-518], and with GUVs by Rigaud, Bassereau, Lévy, and coworkers [519-525].

Although the general concepts for transferring membrane proteins from their native environments into the membranes of artificial vesicles are straightforward, details of the procedures are important to elaborate if one aims at retaining the membrane proteins' activities. An optimization is required for each type of protein. One of the procedures for membrane protein reconstitution in LUVs is shown in Figure 38 [518]; approaches for membrane protein reconstitution in GUVs are summarized in Figure 39 [523].

In many of the procedures applied a "mild" micelle-forming detergent was used (for example, sodium cholate, $n$-octyl- $\beta$-D-glucopyranoside, or $n$-dodecyl octaethylene glycol ether, [520]). They allow in an initial step the solubilization of membrane proteins by extracting them from a biological membrane into mixed detergent/membrane lipid micelles (Figure 38). Detailed experiments with $\mathrm{Ca}^{2+}$-ATPase and bacteriorhodopsin showed that a desired orientation of this membrane protein within the bilayer of LUVs could be achieved to a larger extent than statistically expected so that the directional transport of $\mathrm{Ca}^{2+}$ or $\mathrm{H}^{+}$ across egg PC/egg PA (9:1, molar ratio) bilayers of LUVs from the external medium into the trapped vesicle volume was possible [517,519,520].

For the preparation of proteoliposomes with asymmetric lipid membranes, the preparation of asymmetric LUVs containing membrane-embedded proteins was shown to be possible by an exchange of some of the outer lipids after membrane protein reconstitution by using cyclodextrin [526]; see also Section 4.4. With such procedure, the preparation of sophisticated cell membrane-mimicking vesicle systems is possible for investigation of the influence of the lipid asymmetry on the activity of membrane proteins. 


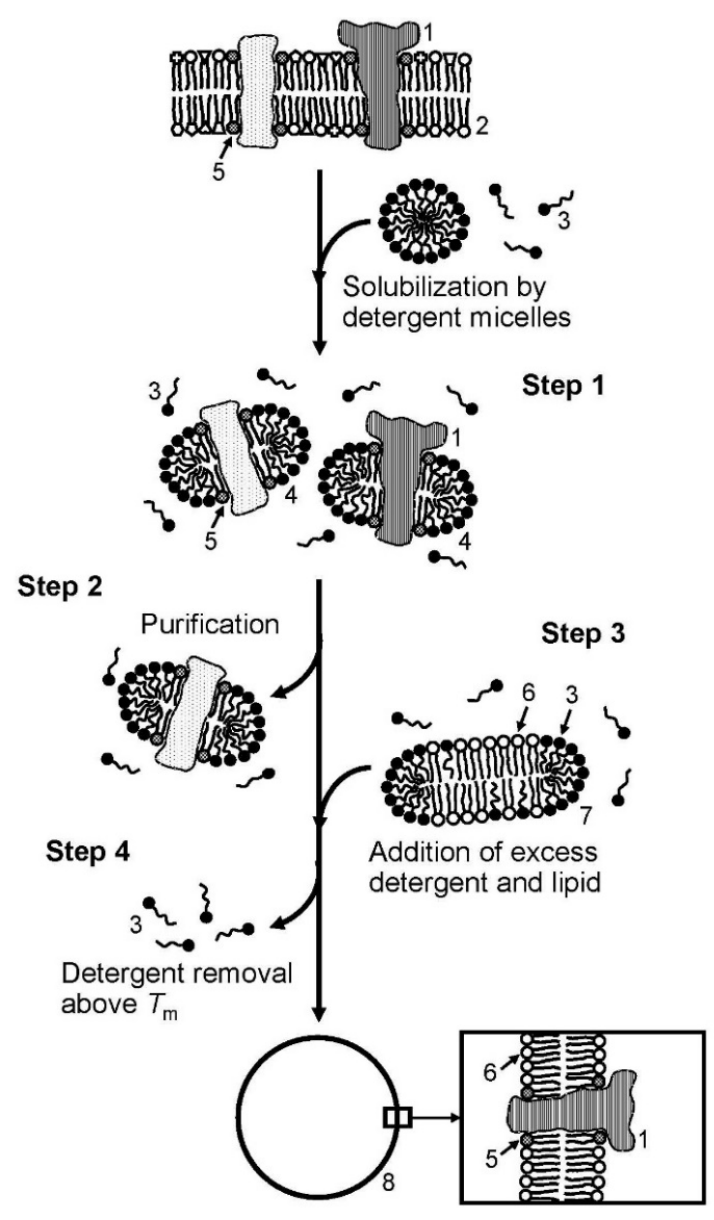

(a)
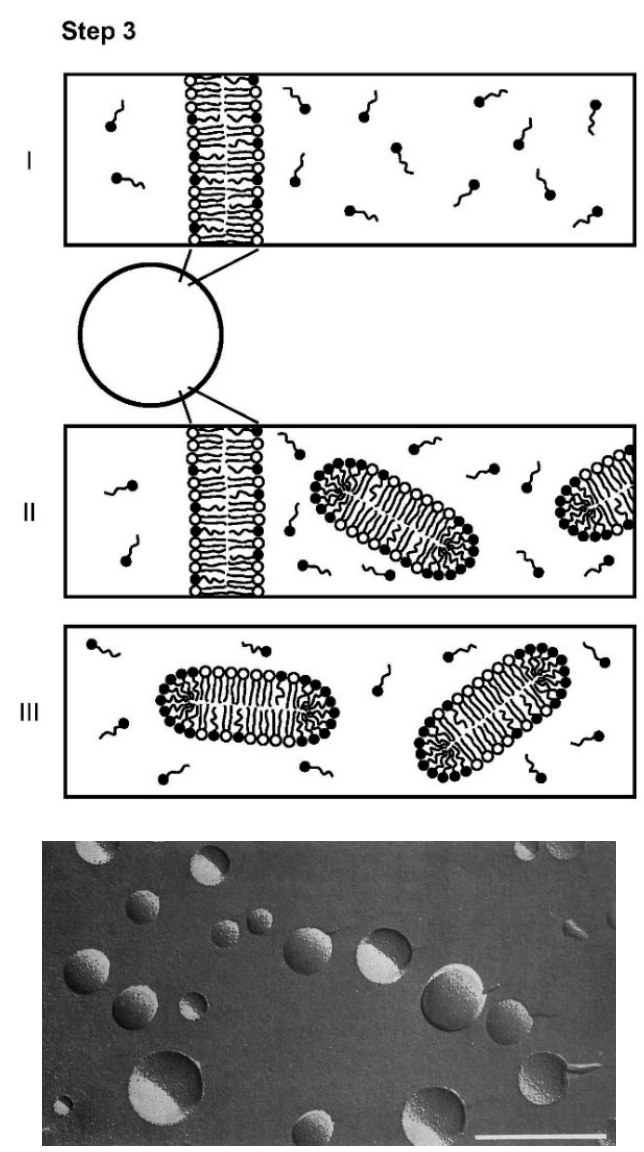

(b)

Figure 38. Membrane protein reconstitution in LUVs. (a) Schematic representation of the concept of one of the procedures that were developed for the preparation of LUVs containing a desired membrane-embedded protein ("proteoliposomes") by using a micelle-forming amphiphile (detergent). The procedure consists of four main steps, as described by Rigaud and collaborators [517,521]. Step 1: The membrane protein (1), which originally was present in a biological membrane (2), is first solubilized with detergent molecules (3) as mixed micelles (4). The mixed micelles contain some of the biomembrane lipids (5), and the mixed micelles are in dynamic equilibrium with non-associated detergent molecules. After purification (step 2), excess bilayer-forming lipids (6) and detergents (3) are first added (step 3, the case of added mixed lipid-detergent micelles is shown (7); see (b) for other possibilities). Step 4: The detergent molecules are then removed (by polystyrene bead adsorption, dialysis, size exclusion chromatography or dilution) to yield lipid vesicles with the desired membrane protein embedded within the vesicle bilayer (8). (b) Top: The three possibilities for adding excess detergent and lipid to a solubilized and purified membrane protein; see step 3 in (a). I: Mixed lipid-detergent vesicles that are saturated with detergent molecules, existing in equilibrium with non-associated detergent molecules. II: Mixed lipid-detergent vesicles that are saturated with detergent molecules, coexisting with mixed detergent-lipid micelles, existing in equilibrium with non-associated detergent molecules. III: Mixed detergent-lipid micelles, existing in equilibrium with non-associated detergent molecules. Please note that in all these cases, the concentration of non-associated lipid molecules is assumed to be negligibly small. This assumption holds for conventional bilayer-forming phospholipids like POPC, CVC(POPC) 10 ${ }^{-10} \mathrm{M}[127,128]$ (see Section 2.15). The drawings are slightly modified versions of previously published figures, [518]. Bottom: Freeze-fracture electron microscopy image of LUVs containing membrane-embedded bacteriorhodopsin from Halobacterium halobium (bar: $500 \mathrm{~nm}$ ); see [516]. The sample was prepared from a 9:1 molar mixture of egg PC and egg PA without any detergent, but instead by using the "reverse-phase evaporation method" (see Figure 17a), followed by polycarbonate membrane extrusion using membranes with average pore diameters of first 400 and then $200 \mathrm{~nm}$, yielding LUVs with a relatively broad size distribution centered around $150 \mathrm{~nm}$. High resolution images show that about 20-200 membrane-embedded proteins are present per LUV, depending on the protein to lipid ratio used; see [516]. Reproduced with small modifications from [191], American Scientific Publishers, 2004; and with permission from [516], Elsevier, 1987. 


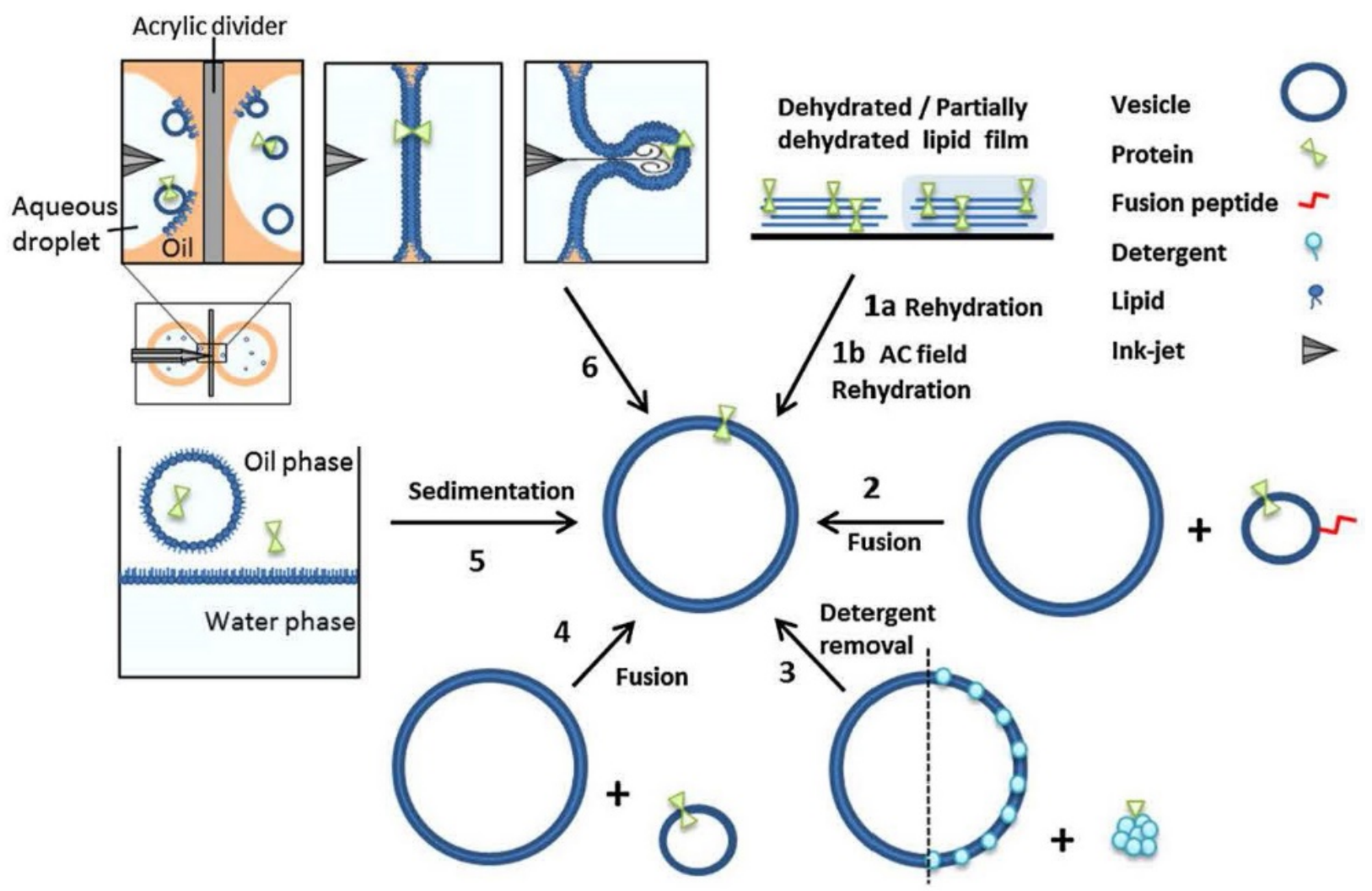

Figure 39. Membrane protein reconstitution in GUVs. Schematic representation of the methods currently used for the preparation of GUVs containing membrane-embedded proteins ("proteo GUVs"), as summarized by Jørgensen et al. (2017) [523]. Some of the methods sketched are related to the methods summarized in Figure 17b for the preparation of membrane protein-free GUVs (drawing is not to scale). (1a) Spontaneous swelling and (1b) electroformation: Rehydration of dehydrated or partially hydrated biological membranes, use of pre-formed "proteoliposomes" or lipid/protein solutions without (1a) or with (1b) applying an external alternating electric field. (2) Vesicle fusion between a preformed GUV and "proteoliposomes" containing membrane fusion-inducing peptides. (3) Addition of a lipid/detergent/protein complex to preformed GUVs. (4) Spontaneous fusion of "proteoliposomes" with preformed GUVs. (5) Application of the "droplettransfer method" with the membrane protein being dissolved in the aqueous interior volume of the initial w/o-droplets or dissolved in the "oil". (6) Application of the "microfluidic jetting method" involving the use of "proteoliposomes". See [523] for details. Reproduced with permission from [523], Springer Nature, 2017.

In the work of Yao et al. (2020) [527], the multidrug-resistant transporter AcrB from Escherichia coli was reconstituted in POPC vesicles by using the detergent $n$-decyl- $\beta$-Dmaltopyranoside and size exclusion chromatography for detergent removal, followed by polycarbonate membrane extrusion using $100 \mathrm{~nm}$ membranes with an average pore diameter of $100 \mathrm{~nm}$. The proteoliposomes obtained had diameters of about $50 \mathrm{~nm}$ (or even smaller), and they could be used successfully for the determination of the structure of AcrB at $3.9 \AA$ resolution by "single particle cryo-electron microscopy, without any need for protein crystallization; see Figure 40 [527]. AcrB is a homo-trimer (with $\mathrm{M} \sim 350 \mathrm{kDa}$ ), with a well known crystal structure, [528]. The challenge of the proteoliposome work was to gain insight into the conformation of AcrB in a membrane-mimicking environment, i.e., a lipid vesicle membrane. Interestingly, roughly $98 \%$ of the ArcB proteins had the same orientation in POPC vesicle membranes, with the water-soluble part of the protein facing into the aqueous interior of the vesicles [527]. This remarkable work provides the grounds for future structural investigations of (large enough) reconstituted membrane proteins, for example in the presence of transmembrane gradients. 


\section{5,757 micrographs}
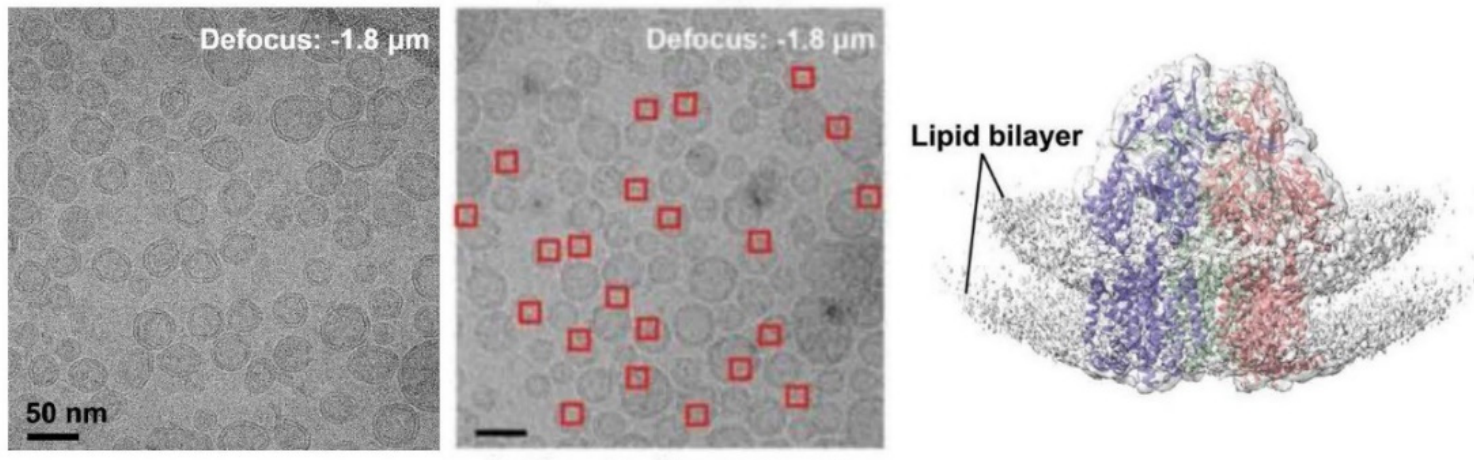

AcrB proteoliposomes on graphene grid

Figure 40. Application of LUVs for the reconstitution of membrane proteins. Determination of the structure of a vesicle membrane-embedded protein by cryo-electron microscopy, as published by Yao et al. (2020) [527]. Left: Cryo-TEM image of POPC vesicles containing membrane-embedded multidrug-resistant transporter AcrB from Escherichia coli, prepared with the help of the detergent $n$-decyl- $\beta$-D-maltopyranoside and polycarbonate membrane extrusion; see text and [527]. Middle: Example for the selection from a total of 5757 recorded movies of AcrB protein particles embedded in the vesicle membranes that could be used for the analysis of the protein structure. Right: Visualization of the structure of AcrB within the bilayer of a vesicle with the localization of the vesicle bilayer. See [527] for details. Reproduced from [527], copyright (C) Yao et al.

To provide a lipidic environment that is as similar as possible to the native lipid matrix of the biomembrane in which a membrane protein of interest is localized, membrane protein reconstitutions can be made by using mixture of lipids to mimic the lipid matrix of the native plasma membrane as close as possible, or cell-derived LUVs or GUVs can be prepared [529-534]. In the latter case, the vesicle membrane will consist not only of an ill-defined mixture of lipids but also of membrane proteins and other components (e.g., cholesterol) that are associated to the membrane. Another possibility is to use exosomes, a type of extracellular vesicles with average diameters in the size range of LUVs [535-539].

Depending on the analytical methods used for analyzing the structure and activity of reconstituted membrane proteins, bicelles [540-542], or cubic phases [543-546] instead of vesicles can also be used successfully as alternative biomembrane-mimicking lipid aggregates.

Proteins and other functional biomacromolecules (DNA, RNA) and small molecules can be entrapped inside GUVs, including entire cell-free ribosomal gene expression systems [33,37,39,543-545]. A somewhat logical next step would be to prepare-from bottom-up-functionalized, cell-like vesicle systems, which also host membrane proteins. Although this is a very ambitious goal, it attracts the attention of a lot of research groups [34,38,546-557]. Although the preparation of living "synthetic cells" (also called "artificial cells) is not (yet) possible, this field of research often is placed within the area of "synthetic biology", with the aim of preparing a minimal system - a "minimal cell" - which one would call living. Despite the building of a "bottom-up" system that one would consider as alive not having been achieved (yet), one of the critical issues is the difficulty of formulating a generally accepted definition of a "living system", of "life" at large. Nevertheless, more and more sophisticated, functionalized vesicles are being prepared that have certain characteristic features of biological cells; see Figure 41 [37,550,558-560]. Although those experiments in which "synthetic cells" are obtained by a "top-down approach", i.e., by putting together selected components present in contemporary cells, or by modifying existing cells (engineering the genome) [561], certainly are remarkable, the making of cells from a mixture of molecules that spontaneously assemble into a functioning cell-like system (i.e., "bottom-up") is, however, much more demanding. 


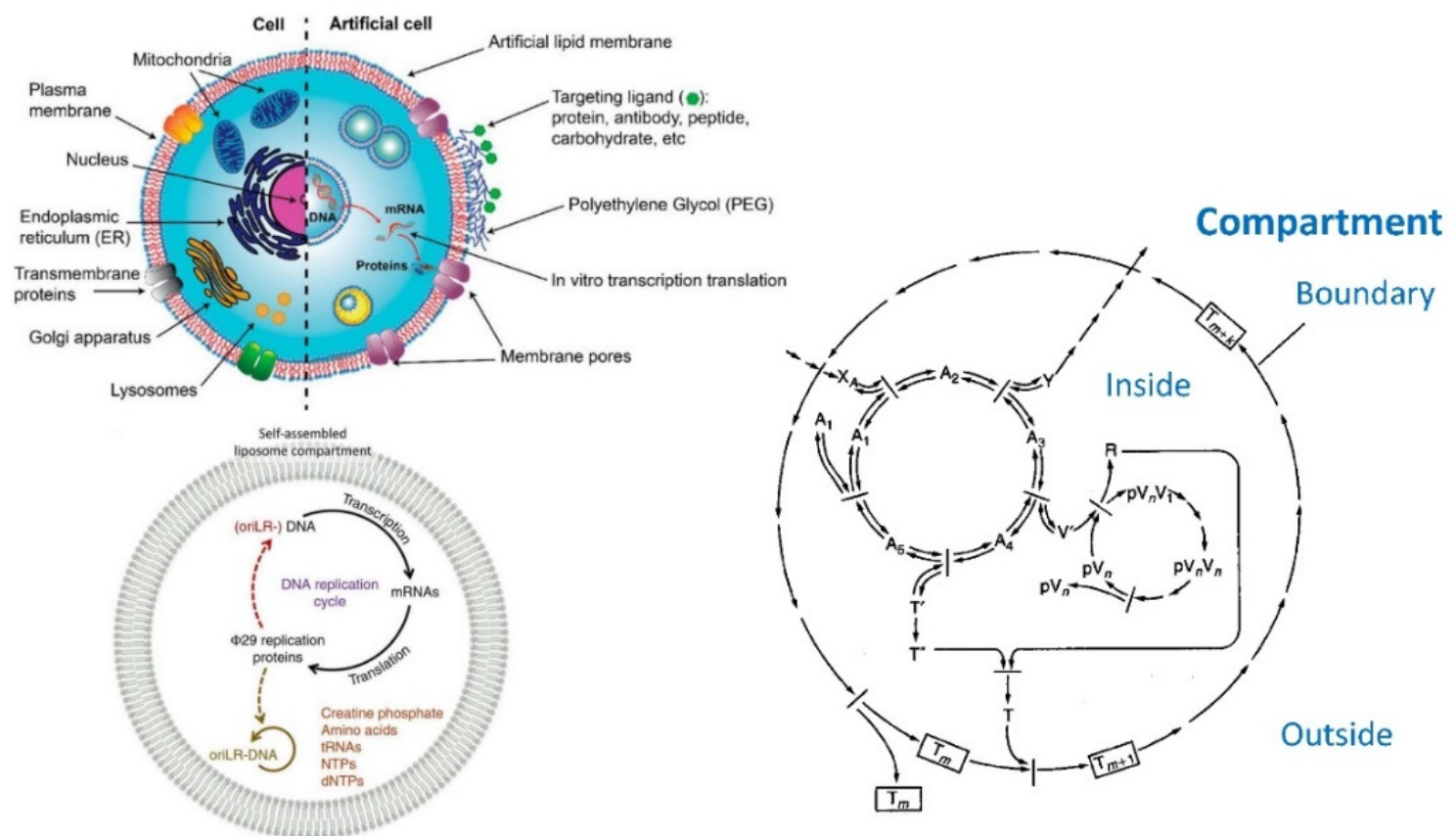

Figure 41. Attempts to prepare vesicle-based cell-like compartment systems "bottom-up". Left, top: Conceptual comparison of a biological cell and a cell-like vesicle system for possible in vivo applications, as published by Trantidou et al. (2017) [550]. The membrane of the cell-like vesicle system is engineered by specific functionalization on the surface (PEGylation and targeting units; see Figures $16 \mathrm{~b}$ and 22. The insertion of protein pores act as gateways, and membrane-bound subcompartments inside the vesicle can segregate content and perform distinct functions, such as in vitro transcription and translation. Left, bottom: Schematic representation of intra-vesicular DNA self-replication and the transformation of the genetic information from DNA to mRNA, which results in the expression of proteins for which the DNA sequence codes (the genome of bacteriophage \$29), achieved with the cell-free protein expression system PURE ("protein synthesis using recombinant elements) system [562,563], as published by van Nies et al. (2018) [37]. Right: The "chemoton" minimal model for the chemical organization of a living system, as proposed and published by Gánti (1997) [560,561]. The "chemoton" model consists of two coupled intra-vesicular cycles, a metabolic cycle (left) and a genetic cycle (right), and a membranous boundary. Reproduced with permission from [550], American Chemical Society, 2017; from [37], Springer Nature, 2018; and from [558], Elsevier, 1997.

The complexity of all known contemporary biological cells is so high that such cells cannot be built "bottom-up". It is not the structural complexity and the molecular composition as such that is difficult to build from the components, even if one would have all molecules required at hand; it is the dynamics of all living cells. There are constantly chemical transformations that occur with high specificity in a spatially and timely highly controlled way, and it is the growth and division propensity of dividing cells and the ability to mutate and to grow and divide in a mutated state.

Although there is no generally accepted definition of life [564-569], there are a number of features that are common to all known forms of life [35]. One of these features is that life is cell-based. Therefore, the overall structural feature of all cells corresponds to the characteristics of a (giant) unilamellar vesicle. For this reason, vesicles not only are the most cell-like compartment system that are used for trying to prepare "artificial cells", but they are also considered as likely precursor structures of the first living cells (so-called "protocells" [35,67,354,357,359-361,363]) that might have formed in prebiotic times and then transformed into the first living cells, at the origin of life. Whether the first cells indeed emerged from pre-biological vesicular systems is not known at all and certainly will remain a fundamental research question and experimental challenge for the future. Only if one would be able to convincingly show that a simple form of a living system can be made "bottom-up", one would have a reasonable idea about how first life may have emerged from non-living forms of matter. It seems that we are still very far away from this. 
A valuable conceptual framework to consider when moving towards the ultimate goal certainly is the one developed by Gánti [558,559] — called "chemoton", Figure 41, right) and the theory of autopoiesis formulated by Maturana and Varela [570] and extended to chemical systems by Luisi $[360,571,572]$.

\section{Concluding Remarks}

In this review we wanted to recall that the aggregation behavior of amphiphilic lipids in the presence of small or large amounts of an aqueous solution not only is fascinating, often complex and challenging to investigate, and relevant for a better understanding of certain features of living forms of matter; dispersed aqueous aggregates of amphiphilic lipids are integral parts in many applications, most notably in biomedical and cosmetic products.

Many applications of lipid aggregates emerged from detailed fundamental investigations. They made it obvious that chemical, physical, biological, as well as engineering aspects need to be taken into account in highly interdisciplinary approaches. Without considering details of the chemical structure of amphiphilic lipids present in biological membranes, for example, and without trying to understand why the particular chemical structures of these lipids are suitable for the function the lipids have in the membrane, or without understanding how such lipidic compounds could be modified chemically for obtaining compounds that have improved properties for a desired application, it is difficult to find reasonable solutions for challenges that one might encounter in application-oriented research. The same can be said about the importance of the physico-chemical properties of lipids (for example, the acidity or basicity of functional groups or the melting temperature), the interaction with biological systems and the environment (e.g., biocompatibility and sustainability), and the way a desired lipid aggregate system is prepared, i.e., how the components of a desired system are assembled reproducibly and most efficiently (the engineering part). Finally, knowledge about the many methods that can be used for analyzing lipid aggregates in dispersed states is important in terms of physical principle of the method, practical use and strength as well as limitations.

Although the focus of the review is on biological amphiphilic lipids, the key concepts concerning the aggregation behavior of fully synthetic, non-natural amphiphiles, including amphiphilic block copolymers, are the same as the ones of biological amphiphiles. Further complexity may originate (i) from amphiphiles that have chemical structures that clearly deviate from the ones present in biological systems; (ii) from complex mixtures of amphiphiles, independent from whether they are of biological origin or non-natural; or (iii) from mixtures of biological and non-natural amphiphiles. Although increased complexity is expected to result in increased economic challenges, it remains to be seen whether the development of the many innovative ideas for applications will ever end in products that can be commercialized for the benefit of the society and the environment.

Author Contributions: Conceptualization, writing and editing, P.W. and S.I. All authors have read and agreed to the published version of the manuscript.

Funding: There was no special funding for writing this review.

Acknowledgments: The authors thank Robert Smith (Park University and Science Advisor U.S. FDA), Pasquale Stano (Università del Salento, Lecce, Italy), and Nemanja Cvjetan (D-MATL, ETH Zürich) for valuable comments on the manuscript. This review was written on the basis of teaching activities of P.W. and S.I. at ETH Zürich and University of Tsukuba (UT), respectively, and the authors' teaching involvement in the Tsukuba Life Science Innovation (T-LSI) Program at UT for master and doctoral students.

Conflicts of Interest: The authors declare no conflict of interest. 


\section{References}

1. Akinc, A.; Maier, M.A.; Manoharan, M.; Fitzgerald, K.; Jayaraman, M.; Barros, S.; Ansell, S.; Du, X.; Hope, M.J.; Madden, T.D.; et al. The Onpattro story and the clinical translation of nanomedicines containing nucleic acid-based drugs. Nat. Nanotechnol. 2019, 14, 1084-1087. [CrossRef]

2. Kulkarni, J.A.; Witzigmann, D.; Thomson, S.B.; Chen, S.; Leavitt, B.R.; Cullis, P.R.; van der Meel, R. The current landscape of nucleic acid therapeutics. Nat. Nanotechnol. 2021, 16, 630-643. [CrossRef] [PubMed]

3. Buschmann, M.D.; Carrasco, M.J.; Alishetty, S.; Paige, M.; Alameh, M.G.; Weissman, D. Nanomaterial Delivery Systems for mRNA Vaccines. Vaccines 2021, 9, 65. [CrossRef]

4. Park, K.S.; Sun, X.; Aikins, M.E.; Moon, J.J. Non-viral COVID-19 vaccine delivery systems. Adv. Drug Deliv. Rev. 2021, 169, 137-151. [CrossRef] [PubMed]

5. Schoenmaker, L.; Witzigmann, D.; Kulkarni, J.A.; Verbeke, R.; Kersten, G.; Jiskoot, W.; Crommelin, D.J.A. mRNA-lipid nanoparticle COVID-19 vaccines: Structure and stability. Int. J. Pharm. 2021, 601, 120586. [CrossRef] [PubMed]

6. Mannino, R.J.; Allebach, E.S.; Strohl, W.A. Encapsulation of High Molecular Weight DNA in Large Unilamellar Phospholipid Vesicles. FEBS Lett. 1979, 101, 229-232. [CrossRef]

7. Wilson, T.; Papahadjopoulos, D.; Taber, R. The Introduction of Poliovirus RNA into Cells via Lipid Vesicles (Liposomes). Cell 1979, 17, 77-84. [CrossRef]

8. Dimitriadis, G.J. Entrapment of plasmid DNA in liposomes. Nucleic Acids Res. 1979, 6, 2697-2705. [CrossRef]

9. Straubinger, R.M.; Papahadjopoulos, D. Liposomes as Carriers for Intracellular Delivery of Nucleic Acids. Methods Enzymol. 1983, 101, 512-527. [PubMed]

10. Gershon, H.; Ghirlando, R.; Guttman, S.B.; Minsky, A. Mode of Formation and Structural Features of DNA-Cationic Liposome Complexes Used for Transfection. Biochemistry 1993, 32, 7143-7151. [CrossRef] [PubMed]

11. Sternberg, B.; Sorgi, F.L.; Huang, L. New structures in complex formation between DNA and cationic liposomes visualized by freeze-fracture electron microscopy. FEBS Lett. 1994, 356, 361-366. [CrossRef]

12. Gustafsson, J.; Arvidson, G.; Karlsson, G.; Almgren, M. Complexes between cationic liposomes and DNA visualized by cryo-TEM. Biochim. Biophys. Acta 1995, 1235, 305-312. [CrossRef]

13. Gregoriadis, G.; Saffie, R.; Hart, S.L. High Yield Incorporation of Plasmid DNA within Liposomes: Effect on DNA Integrity and Transfection Efficiency. J. Drug. Target. 1996, 3, 469-475. [CrossRef] [PubMed]

14. Monnard, P.-A.; Oberholzer, T.; Luisi, P.L. Entrapment of nucleic acids in liposomes. Biochim. Biophys. Acta 1997, 1329, 39-50. [CrossRef]

15. Perrie, Y.; Gregoriadis, G. Liposome-entrapped plasmid DNA: Characterisation studies. Biochim. Biophys. Acta 2000, 1475, 125-132. [CrossRef]

16. Deamer, D.W. From “Banghasomes" to liposomes: A memoir of Alec Bangham, 1921-2010. FASEB J. 2010, 24, 1308-1310. [CrossRef]

17. Heap, B.; Gregoriadis, G. Alec Douglas Bangham. Biogr. Mems Fell. R. Soc. 2011, 57, 25-43. [CrossRef]

18. Israelachvili, J.N. Intermolecular and Surface Forces, 3rd ed.; Elsevier: Amsterdam, The Netherlands, 2011.

19. Kučerka, N.; Nieh, M.-P.; Katsaras, J. Fluid phase lipid areas and bilayer thicknesses of commonly used phosphatidylcholines as a function of temperature. Biochim. Biophys. Acta 2011, 1808, 2761-2771. [CrossRef]

20. Blocher, M.; Walde, P.; Dunn, I.J. Modeling of Enzymatic Reactions in Vesicles: The Case of $\alpha$-Chymotrypsin. Biotechnol. Bioeng. 1999, 62, 36-43. [CrossRef]

21. Torchilin, V.P. Recent Advances with Liposomes as Pharmaceutical Carriers. Nat. Rev. Drug Discov. 2005, 4, 145-160. [CrossRef]

22. Allen, T.M.; Cullis, P.R. Liposomal drug delivery systems: From concept to clinical applications. Adv. Drug Deliv. Rev. 2013, 65, 36-48. [CrossRef] [PubMed]

23. Sercombe, L.; Veerati, T.; Moheimani, F.; Wu, S.Y.; Sood, A.K.; Hua, S. Advances and Challenges of Liposome Assisted Drug Delivery. Front. Pharmacol. 2015, 6, 286. [CrossRef] [PubMed]

24. Gregoriadis, G. Liposome research in drug delivery: The early days. J. Drug Target. 2008, 16, 520-524. [CrossRef] [PubMed]

25. Dawidczyk, C.M.; Kim, C.; Park, J.H.; Russell, L.M.; Lee, K.H.; Pomper, M.G.; Searson, P.C. State-of-the-art in design rules for drug delivery platforms: Lessons learned from FDA-approved nanomedicines. J. Control. Release 2014, 187, 133-144. [CrossRef] [PubMed]

26. Bouwstra, J.A.; Honeywell-Nguyen, P.L.; Gorris, G.S.; Ponec, M. Structure of the skin barrier and its modulation by vesicular formulations. Prog. Lipid Res. 2003, 42, 1-36. [CrossRef]

27. Cevc, G. Lipid vesicles and other colloids as drug carriers on the skin. Adv. Drug Deliv. Rev. 2004, 56, 675-711. [CrossRef] [PubMed]

28. El Maghraby, G.M.; Barry, B.W.; Williams, A.C. Liposomes and skin: From drug delivery to model membranes. Eur. J. Pharm. Sci. 2008, 34, 203-222. [CrossRef] [PubMed]

29. Cevc, G.; Vierl, U. Nanotechnology and the transdermal route. A state of the art review and critical appraisal. J. Control. Release 2010, 141, 277-299. [CrossRef]

30. Kaul, S.; Gulkati, N.; Verma, D.; Mukherjee, S.; Nagaich, U. Role of Nanotechnology in Cosmeceuticals: A Review of Recent Advances. Hindawi J. Pharm. 2018, 3420204. [CrossRef] [PubMed] 
31. Tran, V.V.; Moon, J.-Y.; Lee, Y.-C. Liposomes for delivery of antioxidants in cosmeceuticals: Challenges and development strategies. J. Control. Release 2019, 300, 114-140. [CrossRef] [PubMed]

32. Souto, E.B.; Fernandes, A.R.; Martins-Gomes, C.; Coutinho, T.E.; Durazzo, A.; Lucarini, M.; Souto, S.B.; Silva, A.M.; Santini, A. Nanomaterials for Skin Delivery of Cosmeceuticals and Pharmaceuticals. Appl. Sci. 2020, 10, 1594. [CrossRef]

33. Noireaux, V.; Libchaber, A. A vesicle bioreactor as a step toward an artificial cell assembly. Proc. Natl. Acad. Sci. USA 2004, 101, 17669-17674. [CrossRef] [PubMed]

34. Luisi, P.L.; Stano, P. Approaches to semi-synthetic minimal cells: A review. Naturwissenschaften 2006, 93, 1-13. [CrossRef] [PubMed]

35. Walde, P. Building artificial cells and protocell models: Experimental approaches with lipid vesicles. BioEssays 2010, 32, 296-303. [CrossRef] [PubMed]

36. Elani, Y. Construction of membrane-bound artificial cells using microfluidics: A new frontier in bottom-up synthetic biology. Biochem. Soc. Trans. 2016, 44, 723-730. [CrossRef] [PubMed]

37. van Nies, P.; Westerlaken, I.; Blanken, D.; Salas, M.; Mencía, M.; Danelon, C. Self-replication of DNA by its encoded protens in liposome-based synthetic cells. Nat. Commun. 2018, 9, 1583. [CrossRef] [PubMed]

38. Göpfrich, K.; Platzman, I.; Spatz, J.P. Mastering Complexity: Towards Bottom-up Construction of Multifunctional Eukaryotic Synthetic Cells. Trends Biotechnol. 2018, 36, 938-951. [CrossRef]

39. Stano, P. Gene Expression Inside Liposomes: From Early Studies to Current Protocols. Chem. Eur. J. 2019, 25, 7798-7814. [CrossRef]

40. Dijkgraaf, R. Knowledge Is Infrastructure. Sci. Am. 2017, 316, 8. [CrossRef]

41. Small, D.M. The Physical Chemistry of Lipids (Handbook of Lipid Research); Hanahan, D.J., Ed.; Plenum Press: New York, NY, USA, 1986; Volume 4.

42. Jones, M.N.; Chapman, D. Micelles, Monolayers, and Biomembranes; WILEY-LISS: New York, NY, USA, 1995.

43. Holmberg, K.; Jönsson, B.; Kronberg, B.; Lindman, B. Surfactants and Polymers in Aqueous Solution, 2nd ed.; John Wiley \& Sons: Chichester, UK, 2003.

44. Mahieu, N.; Canet, D.; Cases, J.M.; Boubel, J.C. Micellization of Sodium Oleate in $\mathrm{D}_{2} \mathrm{O}$ As Probed by Proton Longitudinal Magnetic Relaxation and Self-Diffusion Measurements. J. Phys. Chem. 1991, 95, 1844-1846. [CrossRef]

45. Bergstrand, N.; Edwards, K. Aggregate Structure in Dilute Dispersions of Phospholipids, Fatty Acids, and Lysophospholipids. Langmuir 2001, 17, 3245-3253. [CrossRef]

46. Vorum, H.; Brodersen, R.; Kragh-Hansen, U.; Pedersen, A.O. Solubility of long-chain fatty acids in phosphate buffer at pH 7.4. Biochim. Biophys. Acta 1992, 1126, 135-142. [CrossRef]

47. Antunes, F.E.; Coppola, L.; Gaudio, D.; Nicotera, I.; Oliviero, C. Shear rheology and phase behavior of sodium oleate/water mixtures. Colloids Surf. A 2007, 297, 95-104. [CrossRef]

48. Vold, R.D. The Phase Rule Behavior of Concentrated Aqueous Systems of a Typical Colloidal Electrolyte: Sodium Oleate. J. Phys. Chem. 1939, 43, 1213-1231. [CrossRef]

49. McBain, J.W.; Sierichs, W.C. The Solubility of Sodium and Potassium Soaps and the Phase Diagrams of Aqueous Potassium Soaps. I. Am. Chem. Oil Chem. Soc. 1948, 25, 221-225. [CrossRef]

50. Suga, K.; Kondo, D.; Otsuka, Y.; Okamoto, Y.; Umakoshi, H. Characterization of Aqueous Oleic Acid/Oleate Dispersions by Fluorescent Probes and Raman Spectroscopy. Langmuir 2016, 32, 7606-7612. [CrossRef]

51. Edwards, K.; Silvander, M.; Karlsson, G. Aggregate Structure in Dilute Aqueous Dispersions of Oleic Acid/Sodium Oleate and Oleic Acid/Sodium Oleate/Egg Phosphaidylcholine. Biochim. Biophys. Acta 1995, 1126, 135-142.

52. Tatini, D.; Raudino, M.; Ambrosio, M.; Carretti, E.; Davidovich, I.; Talmon, Y.; Ninham, B.W.; LoNostro, P. Physicochemical characterization of green sodium oleate-based formulations. Part 1. structure and rheology. J. Colloid Interface Sci. 2021, 590, 238-248. [CrossRef] [PubMed]

53. Rosevear, F.B. Liquid Crystals: The Mesomorphic Phases of Surfactant Compositions. J. Soc. Cosmet. Chem. 1968, 19, 581-594.

54. McBain, J.W.; Stewart, A. Conductivity in the Three-component System Oleic Acid-Potassium Oleate-Water. J. Chem. Soc. 1933, 928-932. [CrossRef]

55. Small, D.M. A Classification of Biological Lipids Based upon Their Interaction in Aqueous Systems. J. Am. Oil Chem. Soc. 1968, 45, 108-119. [CrossRef] [PubMed]

56. Engblom, J.; Engström, S.; Fontell, K. The effect of the skin penetration enhancer Azone ${ }^{\circledR}$ on fatty acid-sodium soap-water mixtures. J. Control. Release 1995, 33, 299-305. [CrossRef]

57. Mele, S.; Söderman, O.; Ljusberg-Wahrén, H.; Thuresson, K.; Monduzzi, M.; Nylander, T. Phase behavior in the biologically important oleic acid/sodium oleate/water system. Chem. Phys. Lipids 2018, 211, 30-36. [CrossRef]

58. Koynova, R.; Tenchov, B. Phase Transitions and Phase Behavior of Lipids. In Encyclopedia of Biophysics; Roberts, G.C.K., Ed.; European Biophysical Societies Association (EBSA); Springer: Berlin/Heidelberg, Germany, 2013; pp. 1841-1854.

59. Han, S. Molecular dynamics simulation of oleic acid/oleate bilayers: An atomistic model for a ufasome membrane. Chem. Phys. Lipids 2013, 175-176, 79-83. [CrossRef]

60. Gebicki, J.M.; Hicks, M. Ufasomes are Stable Particles surrounded by Unsaturated Fatty Acid Membranes. Nature 1973, 243, 232-234. [CrossRef] [PubMed]

61. Hargreaves, W.R.; Deamer, D.W. Liposomes from Ionic, Single-Chain Amphiphiles. Biochemistry 1978, 17, 3759-3768. [CrossRef] 
62. Cistola, D.P.; Hamilton, J.A.; Jackson, D.; Small, D.M. Ionization and Phase Behavior of Fatty Acids in Water: Application of the Gibbs Phase Rule. Biochemistry 1988, 27, 1881-1888. [CrossRef]

63. Walde, P.; Wick, R.; Fresta, M.; Mangone, A.; Luisi, P.L. Autopoietic Self-Reproduction of Fatty Acid Vesicles. J. Am. Chem. Soc. 1994, 116, 11649-11654. [CrossRef]

64. Chen, I.A.; Szostak, J.W. A Kinetic Study of the Growth of Fatty Acid Vesicles. Biophys. J. 2004, 87, 988-998. [CrossRef] [PubMed]

65. Walde, P.; Namani, T.; Morigaki, K.; Hauser, H. Formation and Properties of Fatty Acid Vesicles (Liposomes). In Liposome Technology, 3rd ed.; Gregoriadis, G., Ed.; Informa Healthcare: New York, NY, USA, 2006; Volume I, pp. 1-19.

66. Morigaki, K.; Walde, P. Fatty Acid Vesicles. Curr. Opin. Colloid Interface Sci. 2007, 12, 75-80. [CrossRef]

67. Mansy, S.S.; Szostak, J.W. Thermostability of model protocell membranes. Proc. Natl. Acad. Sci. USA 2008, 105, 13351-13355. [CrossRef] [PubMed]

68. Salentinig, S.; Sagalowicz, L.; Glatter, O. Self-Assembled Structures and $\mathrm{p} K_{\mathrm{a}}$ Value of Oleic Acid in Systems of Biological Relevance. Langmuir 2010, 26, 11670-11679. [CrossRef]

69. Ferreira, D.A.; Bentley, M.V.L.B.; Karlsson, G.; Edwards, K. Cryo-TEM investigation of phase behaviour and aggregate structure in dilute dispersions of monoolein and oleic acid. Int. J. Pharm. 2006, 310, 203-212. [CrossRef] [PubMed]

70. Monnard, P.-A.; Deamer, D.W. Preparation of Vesicles from Nonphospholipid Amphiphiles. Methods Enzymol. 2003, 372, $133-151$.

71. Ganem-Quintanar, A.; Quintanar-Guerrero, D.; Buri, P. Monoolein: A Review of the Pharmaceutical Applications. Drug Dev. Ind. Pharm. 2000, 26, 809-820. [CrossRef]

72. Kulkarni, C.V.; Wachter, W.; Iglesias-Salto, G.; Engelskirchen, S.; Ahualli, S. Monoolein: A magic lipid? Phys. Chem. Chem. Phys. 2011, 13, 3004-3021. [CrossRef]

73. Garti, N.; Libster, D.; Aserin, A. Lipid polymorphism in lyotropic liquid crystals for triggered release of bioactives. Food Funct. 2012, 3, 700-713. [CrossRef] [PubMed]

74. Milak, S.; Zimmer, A. Glycerol monooleate liquid crystalline phases used in drug delivery systems. Int. J. Pharm. 2015, 478, 569-587. [CrossRef] [PubMed]

75. Hyde, S.T.; Andersson, S.; Ericsson, B.; Larsson, K. A cubic structure consisting of a lipid bilayer forming an infinite periodic minimum surface of the gyroid type in the glycerolmonooleat-water system. Z. Kristallogr. 1985, 168, $213-219$.

76. Briggs, J.; Chung, H.; Caffrey, M. The Temperature-Composition Phase Diagram and Mesophase Structure Characterization of the Monoolein/Water System. J. Phys. II 1996, 6, 723-751. [CrossRef]

77. Qiu, H.; Caffrey, M. The phase diagram of the monoolein/water system: Metastability and equilibrium aspects. Biomaterials 2000, 21, 223-234. [CrossRef]

78. Tenchov, B.; Koynova, R. Cubic phases in phosphatidylethanolamine dispersions: Formation, stability and phase transitions. Chem. Phys. Lipids 2017, 208, 65-74. [CrossRef]

79. Borné, J.; Nylander, T.; Khan, A. Vesicle formation and other structures in aqueous dispersions of monoolein and sodium oleate. J. Colloid Interface Sci. 2003, 257, 310-320. [CrossRef]

80. Cong, J.Y.T.; Mulet, X.; Boyd, B.J.; Drummond, C.J. Steric Stabilizers for Cubic Phase Lyotropic Liquid Crystal Nanodispersions (Cubosomes). Adv. Planar Lipid Bilayers Liposomes 2015, 21, 131-187.

81. Borné, J.; Nylander, T.; Khan, A. Phase Behavior and Aggregate Formation for the Aqueous Monoolein System Mixed with Sodium Oleate and Oleic Acid. Langmuir 2001, 17, 7742-7751. [CrossRef]

82. Fong, C.; Zhai, J.; Drummond, C.J.; Tran, N. Micellar Fd3m cubosomes from monoolein—Long chain unsaturated fatty acid mixtures: Stability on temperaure and pH response. J. Colloid Interface Sci. 2020, 566, 98-106. [CrossRef] [PubMed]

83. Nakano, M.; Teshigawara, T.; Sugita, A.; Leesajakul, W.; Taniguchi, A.; Kamo, T.; Matsuoka, H.; Handa, T. Dispersions of Liquid Crystalline Phases of the Monoolein/Oleic Acid/Pluronic F127 System. Langmuir 2002, 18, 9283-9288. [CrossRef]

84. Marsh, D. (Ed.) Phospholipids. In Handbook of Lipid Bilayers, 2nd ed.; CRC Press: Boca Raton, FL, USA, 2013; Section II, subsection II.6; pp. 259-269.

85. Arvidson, G.; Brentel, I.; Khan, A.; Lindblom, G.; Fontell, K. Phase equilibria in four lysophosphatidylcholine/water systems. Exceptional behavior of 1-palmitoyl-glycerophosphocholine. Eur. J. Biochem. 1985, 152, 753-759. [CrossRef]

86. Inoue, K.; Suzuki, K.; Nojima, S. Morphology of Lipid Micelles Containing Lysolecithin. J. Biochem. 1977, 81, 1097-1106. [CrossRef]

87. Mattai, J.; Shipley, G.G. The kinetics of formation and structure of the low-temperature phase of 1-stearoyl-lysophosphatidylcholine. Biochim. Biophys. Acta 1986, 859, 257-265. [CrossRef]

88. Tristram-Nagle, S.; Petrache, H.I.; Nagle, J.F. Strucure and Interactions of Fully Hydrated Dioleoylphosphatidylcholine Bilayers. Biophys. J. 1998, 75, 917-925. [CrossRef]

89. Wiener, M.C.; White, S.H. Structure of a fluid dioleoylphosphatidylcholine bilayer determined by joint refinement of $x$-ray and neutron diffraction data. III: Complete structure. Biophys. J. 1992, 61, 434-447. [CrossRef]

90. Bergenståhl, B.A.; Stenius, P. Phase Diagrams of Dioleoylphosphatidylcholine with Formamide, Methylformamide, and Dimethylformamide. J. Phys. Chem. 1987, 91, 5944-5948. [CrossRef]

91. Hoffmann, I.; Michel, R.; Sharp, M.; Holderer, O.; Appavou, M.-S.; Polzer, F.; Farago, B.; Gradzielski, M. Softening of phospholipid membranes by the adhesion of silica nanoparticles-As seen by neutron spin-echo (NSE). Nanoscale 2014, 6, 6945-6952. [CrossRef] [PubMed]

92. Palacios, L.E.; Wang, T. Egg-Yolk Lipid Fractionation and Lecithin Characterization. J. Am. Oil Chem. Soc. 2005, 82, 571-578. [CrossRef] 
93. Kiełbowicz, G.; Gładkowski, W.; Chojnacka, A.; Wawrzeńczyk, C. A simple method for positional analysis of phosphatidylcholine. Food Chem. 2012, 135, 2542-2548. [CrossRef] [PubMed]

94. Small, D. Phase equilibria and structure of dry and hydrated egg lecithin. J. Lipid Res. 1967, 8, 551-557. [CrossRef]

95. Bangham, A.D.; Horn, R.W. Negative Staining of Phospholipids and their Structural Modification by Surface-active Agents as observed in the Electron Microsope. J. Mol. Biol. 1964, 8, 660-668. [CrossRef]

96. Holzer, M.; Bernert, S.; Momm, J.; Schubert, R. Preparative size exclusion chromatography combined with detergent removal as a versatile tool to prepare unilamellar and spherical liposomes of highly uniform size distribution. J. Chromatogr. A 2009, 1216, 5838-5848. [CrossRef] [PubMed]

97. Bergenståhl, B.; Fontell, K. Phase equilibria in the system soybean lecithin/water. Progr. Colloid Polym. Sci. 1983, 68, 48-52.

98. Lynch, D.V.; Steponkus, P.L. Lyotropic phase behavior of unsaturated phosphatidylcholine species: Relevance to the mechanism of plasma membrane destabilization and freezing injury. Biochim. Biophys. Acta 1989, 984, 267-272. [CrossRef]

99. Koster, K.L.; Webb, M.S.; Bryant, G.; Lynch, D.V. Interactions between soluble sugars and POPC (1-palmitoyl-2-oleoyphosphatidylcholine) during dehydration: Vitrification of sugars alters the phase behavior of the phospholipid. Biochim. Biophys. Acta 1994, 1193, 143-150. [CrossRef]

100. Kučerka, N.; Tristram-Nagle, S.; Nagle, J.F. Structure of Fully Hydrated Fluid Phase Lipid Bilayers with Monounsaturated Chains. J. Membr. Biol. 2005, 208, 193-202. [CrossRef]

101. Koynova, R.; Caffrey, M. Phases and phase transitions of the phosphatidylcholines. Biochim. Biophys. Acta 1998, 1376, 91-145. [CrossRef]

102. O’Neill, S.D.; Leopold, A.D. An Assessment of Phase Transitions in Soybean Membranes. Plant Physiol. 1982, 70, 1405-1409. [CrossRef] [PubMed]

103. Barton, P.G.; Gunstone, F.D. Hydrocarbon Chain Packing and Molecular Motion in Phospholipid Bilayers Formed from Unsaturated Lecithins. Synthesis and Properties of Sixteen Positional Isomers of 1,2-Dioctadecenoyl-sn-glycero-3-phosphorylcholine. J. Biol. Chem. 1975, 250, 4470-4476. [CrossRef]

104. Marsh, D. Thermodynamic Analysis of Chain-Melting Transition Temperatures for Monounsaturated Phospholipid Membranes: Dependence on cis-Monoenoic Double Bond Position. Biophys. J. 1999, 77, 953-963. [CrossRef]

105. Ipsen, J.H.; Mouritsen, O.G.; Zuckermann, M.J. Theory of thermal anomalies in the specific heat of lipid bilayers containing cholesterol. Biophys. J. 1989, 56, 661-667. [CrossRef]

106. Tieleman, D.P.; Berendsen, H.J.C. Molecular dynamics simulations of a fully hydrated dipalmitoylphosphatidylcholine bilayer with different macroscopic boundary conditions and parameters. J. Chem. Phys. 1996, 105, 4871-4880. [CrossRef]

107. Rappolt, M.; Pabst, G.; Rapp, G.; Kriechbaum, M.; Amenitsch, H.; Krenn, C.; Bernstorff, S.; Laggner, P. New evidence for gel-liquid crystalline phase coexistence in the ripple phase of phosphatidylcholines. Eur. Biophys. J. 2000, 29, 125-133. [CrossRef] [PubMed]

108. de Vries, A.H.; Yefimov, S.; Mark, A.E.; Marrink, S.J. Molecular structure of the lecithin ripple phase. Proc. Natl. Acad. Sci. USA 2005, 102, 5392-5396. [CrossRef] [PubMed]

109. El Jastimi, R.; Edwards, K.; Lafleur, M. Characterization of Permeability and Morphological Perturbations Induced by Nisin on Phosphatidylcholine Membranes. Biophys. J. 1999, 77, 842-852. [CrossRef]

110. Farkuh, L.; Hennies, P.T.; Nunes, C.; Reis, S.; Barreiros, L.; Segundo, M.A.; Oseliero Filho, P.L.; Oliveira, C.L.P.; Cassago, A.; Portugal, R.V.; et al. Characterization of phospholipid vesicles containing lauric acid: Physicochemical basis for process and product development. Heliyon 2019, 5, e02648. [CrossRef]

111. Matviykiv, S.; Deyhle, H.; Kohlbrecher, J.; Neuhaus, N.; Zumbuehl, A.; Müller, B. Small-Angle Neutron Scattering Study of Temperature-Induced Structural Changes in Liposomes. Langmuir 2019, 35, 11210-11216. [CrossRef] [PubMed]

112. Doskocz, J.; Dałek, P.; Foryś, A.; Trzebicka, B.; Przybyło, M.; Mesarec, L.; Iglič, A.; Langner, M. The effect of lipid phase on liposome stability upon exposure to the mechanical stress. Biochim. Biophys. Acta 2020, 1862, 183361. [CrossRef] [PubMed]

113. Linblom, G.; Rilfors, L.; Hauksson, J.B.; Brentel, I.; Sjölund, M.; Bergenståhl, B. Effect of Head-Group Structure and Counterion Condensation on Phase Equilibria in Anionic Phospholipid-Water Systems Studied by ${ }^{2} \mathrm{H},{ }^{23} \mathrm{Na}$, and ${ }^{31} \mathrm{P}$ NMR and X-ray Diffraction. Biochemistry 1991, 30, 10938-10948. [CrossRef] [PubMed]

114. Tocanne, J.-F.; Teissié, J. Ionization of phospholipids and phospholipid-supported interfacial lateral diffusion of protons in membrane model systems. Biochim. Biophys. Acta 1990, 1031, 111-142. [CrossRef]

115. Faraudo, J.; Travesset, A. Phosphatidic Acid Domains in Membranes. Effect of Divalent Counterions. Biophys. J. 2007, 92, 2806-2818. [CrossRef]

116. Zhang, T.; Cathcart, G.; Vidalis, A.S.; Allen, H.C. Cation effects on phosphatidic acid monolayers at various $\mathrm{pH}$ conditions. Chem. Phys. Lipids 2016, 200, 24-31. [CrossRef]

117. Hauser, H. Mechanism of spontaneous vesiculation. Proc. Natl. Acad. Sci. USA 1989, 86, 5351-5355. [CrossRef] [PubMed]

118. Nakano, M.; Fukuda, M.; Kudo, T.; Matsuzaki, N.; Azuma, T.; Sekine, K.; Endo, H.; Handa, T. Flip-Flop of Phospholipids in Vesicles: Kinetic Analysis with Time-Resolved Small-Angle Neutron Scattering. J. Phys. Chem. B 2009, 113, 6745-6748. [CrossRef] [PubMed]

119. Kozlov, M.M.; Leikin, S.; Rand, R.P. Bending, Hydration and Interstitial Energies Quantitatively Account for the HexagonalLamellar-Hexagonal Reentrant Phase Transition in Dioleoylphosphatidylethanolamine. Biophys. J. 1994, 67, 1603-1611. [CrossRef]

120. Shyamsunder, E.; Gruner, S.M.; Tate, M.W.; Turner, D.C.; So, P.T.C.; Tilcock, C.P.S. Observation of Inverted Cubic Phase in Hydrated Dioleoylphosphatidylethanolamine Membranes. Biochemistry 1988, 27, 2332-2336. [CrossRef] [PubMed] 
121. Erbes, J.; Czeslik, C.; Hahn, W.; Winter, R.; Rappolt, M.; Rapp, G. On the Existence of Bicontinuous Cubic Phases in Dioleoylphosphatidylethanolamine. Ber. Bunsenges. Phys. Chem. 1994, 98, 1287-1293. [CrossRef]

122. Gawrisch, K.; Parsegain, V.A.; Hajduk, D.A.; Tate, M.W.; Gruner, S.M.; Fuller, N.L.; Rand, R.P. Energetics of the HexagonalLamellar-Hexagonal-Phase Transition Sequence in Dioleoylphosphatidylethanolamine Membranes. Biochemistry 1992, 31, 28562864. [CrossRef] [PubMed]

123. Cistola, D.P.; Small, D.M. On Micelle Formation and Phase Separation. J. Am. Chem. Soc. 1990, 112, 3214-3215. [CrossRef]

124. Romsted, L. Introduction to Surfactant Self-Assembly. In Supramolecular Chemitry: From Molecules to Nanomaterials; Gale, P.A., Steed, J.W., Eds.; Wiley: New York, NY, USA, 2012; Volume 1, 23p.

125. Israelachvili, J.N.; Mitchell, D.J.; Ninham, B.W. Theory of self-assembly of lipid bilayers and vesicles. Biochim. Biophys. Acta 1977, 470, 185-201. [CrossRef]

126. Namani, T.; Walde, P. From Decanoate Micelles to Decanoic Acid/Dodecylbenzenesulfonate Vesicles. Langmuir 2005, 21, 6210-6219. [CrossRef]

127. Smith, R.; Tanford, C. The Critical Micelle Concentration of L- $\alpha$-dipalmitoylphosphatidylcholine in Water and Water/methanol Solutions. J. Mol. Biol. 1972, 67, 75-83. [CrossRef]

128. Buboltz, J.T.; Feigenson, G.W. Phospholipid Solubility Determined by Equilibrium Distribution between Surface and Bulk Phase. Langmuir 2005, 21, 6296-6301. [CrossRef] [PubMed]

129. Laughlin, R.G. The Aqueous Phase Behavior of Surfactants; Academic Press: London, UK, 1994.

130. Alfutimie, A.; Curtis, R.; Tiddy, G.J.T. Lyotropic Surfactant Liquid Crystals: Micellar Systems. In Handbook of Liquid Crystals, 2nd ed.; Goodby, J.W., Collings, P.J., Kato, T., Tschierske, C., Gleeson, H.J., Rynes, P., Eds.; Wiley-VCH Verlag GmbH \& Co. KGaA: Weinheim, Germany, 2014; Volume 6, Chapter 12; pp. 1-44.

131. Kékicheff, P.; Grabielle-Mandelmont, C.; Ollivon, M. Phase Diagram of Sodium Dodecyl Sulfate-Water System. 1. A Calorimetric Study. J. Colloid Interface Sci. 1989, 131, 112-132. [CrossRef]

132. Varade, D.; Aramaki, K.; Stubenrauch, C. Phase diagrams of water-alkyltrimethylammonium bromide systems. Colloids Surf. A 2008, 315, 205-209. [CrossRef]

133. Corcoran, J.; Fuller, S.; Rahman, A.; Shinde, N.; Tiddy, G.J.T.; Attard, G.S. Amphitropic Liquid Crystals. Part 1.-Effect of a Thermotropic Mesogen on Lyotropic Mesomorphism, and of a Surfactant on Thermotropic Mesomorphism. The $\mathrm{C}_{16} \mathrm{EO}_{8}-5$ CB-Water System. J. Mater. Chem. 1992, 2, 695-702. [CrossRef]

134. Strey, R.; Schomäcker, R.; Roux, D.; Nallet, F.; Olsson, U. Dilute Lamellar and $\mathrm{L}_{3}$ Phases in the Binary Water-C ${ }_{12} \mathrm{E}_{5} \mathrm{System}_{\mathrm{s}}$. Chem. Soc. Faraday Trans. 1990, 86, 2253-2261. [CrossRef]

135. Riaz, M.K.; Riaz, M.A.; Zhang, X.; Lin, C.; Wong, K.H.; Chen, X.; Zhang, G.; Lu, A.; Yang, Z. Surface Functionalization and Targeting Strategies of Liposomes in Solid Tumor Therapy: A Review. Int. J. Mol. Sci. 2018, 19, 195. [CrossRef]

136. Ahmed, K.S.; Hussein, S.A.; Ali, A.H.; Korma, S.A.; Lipeng, Q.; Jinghua, C. Liposome: Composition, characterisation, preparation, and recent innovation in clinical applications. J. Drug Target. 2019, 27, 742-761. [CrossRef]

137. Filipczak, N.; Pan, J.; Yalamarty, S.S.K.; Torchilin, V.P. Recent advancements in liposome technology. Adv. Drug Deliv. Rev. 2020, 156, 4-22. [CrossRef] [PubMed]

138. Crommelin, D.J.A.; van Hoogevest, P.; Stom, G. The role of liposomes in clinical nanomedicine development. What now? Now what? J. Control. Release 2020, 318, 256-263. [CrossRef] [PubMed]

139. Barriga, H.M.G.; Holme, M.N.; Stevens, M.M. Cubosomes: The Next Generation of Smart Lipid Nanoparticles? Angew. Chem. Int. Ed. 2019, 58, 2958-2978. [CrossRef] [PubMed]

140. Barauskas, J.; Johnsson, M.; Joabsson, F.; Tiberg, F. Cubic Phase Nanoparticles (Cubosomes): Principles for Controlling Size, Structure, and Stability. Langmuir 2005, 21, 2569-2577. [CrossRef] [PubMed]

141. Barauskas, J.; Johnsson, M.; Tiberg, F. Self-Assembled Lipid Superstructures: Beyond Vesicles and Liposomes. Nano Lett. 2005, 5, 1615-1619. [CrossRef]

142. Larsson, K.; Quinn, P.; Sato, K.; Tiberg, F. Lipids: Structure, Physical Properties and Functionality; The Oily Press, PJ Barnes \& Associates: Bridgwater, UK, 2006.

143. Mertins, O.; Mathews, P.D.; Angelova, A. Advances in the Design of pH-Sensitive Cubosome Liquid Crystalline Nanocarriers for Drug Delivery Applications. Nanomaterials 2020, 10, 963. [CrossRef]

144. Azmi, I.D.M.; Moghimi, S.M.; Yaghmur, A. Cubosomes and hexosomes as versatile platforms for drug delivery. Ther. Deliv. 2015, 6, 1347-1364. [CrossRef] [PubMed]

145. Amar-Yuli, I.; Wachtel, E.; Ben Shoshan, E.; Danino, D.; Aserin, A.; Garti, N. Hexosome and Hexagonal Phases Mediated by Hydration and Polymeric Stabilizer. Langmuir 2007, 23, 3637-3645. [CrossRef]

146. Conn, C.E.; Seddon, J.M. Nonlamellar Lipid Aggregates. In Liposomes, Lipid Bilayers and Model Membranes. From Basic Research to Applications; Pabst, G., Kučerka, N., Nieh, M.-P., Katsaras, J., Eds.; CRC Press, Taylor \& Francis Group: Boca Raton, FL, USA, 2014; Chapter 2; pp. 31-47.

147. Treyer, M.; Walde, P.; Oberholzer, T. Permeability Enhancement of Lipid Vesicles to Nucleotides by Use of Sodium Cholate: Basic Studies and Application to an Enzyme-Catalyzed Reaction Occurring inside the Vesicles. Langmuir 2002, 18, 1043-1050. [CrossRef]

148. Heerklotz, H. Interactions of surfactants with lipid membranes. Q. Rev. Biophys. 2008, 41, 205-264. [CrossRef] [PubMed] 
149. Heerklotz, H.; Blume, A. Detergent Interactions with Lipid Bilayers and Membrane Proteins. In Comprehensive Biophysics; Egelman, E.H., Ed.; Academic Press, Elsevier: Amsterdam, The Netherlands, 2012; Volume 5, pp. 63-91.

150. Lichtenberg, D.; Ahyayauch, H.; Goñi, F.M. The Mechanism of Detergent Solubilization of Lipid Bilayers. Biophys. J. 2013, 105, 289-299. [CrossRef] [PubMed]

151. Arouri, A.; Mouritsen, O.G. Membrane-perturbing effect of fatty acids and lysolipids. Prog. Lipid Res. 2013, 52, 130-140. [CrossRef] [PubMed]

152. Motlaq, V.F.; Ortega-Holmberg, M.; Edwards, K.; Gedda, L.; Lyngsø, J.; Pedersen, J.S.; Bergström, L.M. Investigation of the enhanced ability of bile salt surfactants to solubilize phospholipid bilayers and form mixed micelles. Soft Matter 2021, 17, 7769-7780. [CrossRef]

153. Grit, M.; Crommelin, D.J.A. The effect of aging on the physical stability of liposome dispersions. Chem. Phys. Lipids 1992, 62, 113-122. [CrossRef]

154. Sabín, J.; Prieto, G.; Ruso, J.M.; Hildago-Álvarez, R.; Sarimento, F. Size ad stability of liposomes: A possible role of hydration and osmotic forces. Eur. Phys. J. E 2006, 20, 401-408. [CrossRef] [PubMed]

155. Kato, K.; Walde, P.; Koine, N.; Ichikawa, S.; Ishikawa, T.; Nagahama, R.; Ishihara, T.; Tsujii, T.; Shudou, M.; Omokawa, Y.; et al. Temperature-Sensitive Nonionic Vesicles Prepared from Span 80 (Sorbitan Monooleate). Langmuir 2008, 24, 10762-10770. [CrossRef]

156. Ong, S.G.M.; Chitneni, M.; Lee, K.S.; Ming, L.C.; Yuen, K.H. Evaluation of Extrusion Technique for Nanosizing Liposomes. Pharmaceutics 2016, 8, 36. [CrossRef] [PubMed]

157. Kanásova, M.; Nesměrák, K. Systematic reviews of liposome's characterization methods. Monatsh. Chem. 2017, 148, 1581-1593. [CrossRef]

158. Fan, Y.; Marioli, M.; Zhang, K. Analytical characterization of liposomes and other lipid nanoparticles for drug delivery. J. Pharm. Biomed. Anal. 2021, 192, 113642. [CrossRef] [PubMed]

159. Rand, P.R.; Fuller, N.; Parsegian, V.A.; Rau, D.C. Variation in Hydration Forces between Neutral Phospholipid Bilayers: Evidence for Hydration Attraction. Biochemistry 1988, 27, 7711-7722. [CrossRef]

160. Smirnova, Y.G.; Aeffner, S.; Risselada, H.J.; Salditt, T.; Marrink, S.J.; Müller, M.; Knecht, V. Interbilayer repulsion forces between tension-free lipid bilayers from simulation. Soft Matter 2013, 9, 10705-10718. [CrossRef]

161. New, R.R.C. (Ed.) Preparation of liposomes. In Liposomes. A practical Approach; Oxford University Press: Oxford, UK, 1990; Chapter 2; pp. 33-104.

162. Zuidam, N.J.; van Winden, E.; de Vrueh, R.; Crommelin, D.J.A. Stability, storage, and sterilization of liposomes. In Liposomes. A Practical Approach, 2nd ed.; Torchilin, V., Weissig, V., Eds.; Oxford University Press: Oxford, UK, 2003; Chapter 5; pp. $149-165$.

163. Crommelin, D.J.A. Influence of Lipid Composition and Ionic Strength on the Physical Stability of Liposomes. J. Pharm. Sci. 1984, 73, 1559-1563. [CrossRef] [PubMed]

164. Carrión, F.J.; de la Maza, A.; Parra, J.L. The Influence of Ionic Strength and Lipid Bilayer Charge on the Stability of Liposomes. J. Colloid Interface Sci. 1994, 164, 78-87. [CrossRef]

165. du Plessis, J.; Ramachandran, C.; Weiner, N.; Müller, D.G. The influence of lipid composition and lamellarity of liposomes on the physical stability of liposomes upon storage. Int. J. Pharm. 1996, 127, 273-278. [CrossRef]

166. Armengo, X.; Estelrich, J. Physical stability of different liposome compositions obtained by extrusion method. J. Microencapsulation 1995, 12, 525-535. [CrossRef] [PubMed]

167. Traïkia, M.; Warschawski, D.E.; Recouvreur, M.; Cartaud, J.; Devaux, P.F. Formation of unilamellar vesicles by repetitive freezethaw cycles: Characterization by electron microsocpy and ${ }^{31}$ P-nuclear magnetic resonance. Eur. Biophys. J. 2000, $29,184-195$. [CrossRef] [PubMed]

168. Heurtault, B.; Saulnier, P.; Pech, B.; Proust, J.-E.; Benoit, J.-P. Physico-chemical stability of colloidal lipid particles. Biomaterials 2003, 24, 4283-4300. [CrossRef]

169. Nielsson, C.; Østergaard, J.; Weng Larsen, S.; Larsen, C.; Urtti, A.; Yaghmur, A. PEGylation of Phytantriol-Based Lyotropic Liquid Crystalline Particles-The Effect of Lipid Composition, PEG Chain Length, and Temperature on the Internal Nanostructure. Langmuir 2014, 30, 6398-6407. [CrossRef] [PubMed]

170. Kronberg, B.; Dahlman, A.; Carlfors, J.; Karlsson, J.; Artursson, P. Preparation and Evaluation of Sterically Stabilized Liposomes: Colloidal Stability, Serum Stability, Macrophage Uptake, and Toxicity. J. Pharm. Sci. 1990, 79, 667-671. [CrossRef]

171. Allen, T.M.; Hansen, C.; Martin, F.; Redemann, C.; Yau-Young, A. Liposomes containing synthetic lipid derivatives of poly(ethylene glycol) show prolonged half-lives in vivo. Biochim. Biophys. Acta 1991, 1066, 29-36. [CrossRef]

172. Lasic, D.D.; Needham, D. The "Stealth" Liposome: A Prototypical Biomaterial. Chem. Rev. 1995, 95, 2601-2628. [CrossRef]

173. Čeh, B.; Winterhalter, M.; Frederik, P.M.; Vallner, J.J.; Lasic, D.D. Stealth ${ }^{\circledR}$ liposomes: From theory to product. Adv. Drug Deliv. Rev. 1997, 24, 165-177.

174. Immordino, M.L.; Dosio, F.; Cattel, L. Stealth liposomes: Review of the basic science, rationale, and clinical applications, existing and potential. Int. J. Nanomed. 2006, 1, 297-315.

175. Li, M.; Jiang, S.; Simon, J.; Passlick, D.; Frey, M.-L.; Wagner, M.; Mailänder, V.; Crespy, D.; Landfester, K. Brush Conformation of Polyethylene Glycol Determines the Stealth Effect of Nanocarriers in the Low Protein Adsorption Regime. Nano Lett. 2021, 21, 1591-1598. [CrossRef]

176. Larsson, K. Cubic Lipid-Water Phases: Structure and Biomembrane Aspects. J. Phys. Chem. 1989, 89, 7304-7314. [CrossRef] 
177. Pitto-Barry, A.; Barry, N.P.E. Pluronic ${ }^{\circledR}$ block-copolymers in medicine: From chemical and biological versatility to rationalisation and clinical advances. Polym. Chem. 2014, 5, 3291-3297. [CrossRef]

178. Laughlin, R. Equilibrium vesicles: Fact or fiction? Colloids Surf. A 1997, 128, 27-38. [CrossRef]

179. Lasic, D.D.; Joannic, R.; Keller, B.C.; Frederik, P.M.; Auvray, L. Spontaneous vesiculation. Adv. Colloid Interface Sci. 2001, 89-90, 337-349. [CrossRef]

180. Guida, V. Thermodynamics and kinetics of vesicles formation processes. Adv. Colloid Interface Sci. 2010, 161, 77-88. [CrossRef] [PubMed]

181. Käs, J.; Sackmann, E. Shape transitions and shape stability of giant phospholipid vesicles in pure water induced by area-to-volume changes. Biophys. J. 1991, 60, 825-844. [CrossRef]

182. Lipowsky, R. The morphology of lipid membranes. Curr. Opin. Struct. Biol. 1995, 5, 531-540. [CrossRef]

183. Mui, B.L.-S.; Döbereiner, H.-G.; Madden, T.D.; Cullis, P.R. Influence of Transbilayer Area Asymmetry on the Morphology of Large Unilamellar Vesicles. Biophys. J. 1995, 69, 930-941. [CrossRef]

184. Svetina, S.; Žekš, B. Shape Behavior of Lipid Vesicles as the Basis of Some Cellular Processes. Anat. Rec. 2002, 268, 215-225. [CrossRef] [PubMed]

185. Neuhaus, F.; Mueller, D.; Tanasescu, R.; Balog, S.; Ishikawa, T.; Brezesinski, G.; Zumbuehl, A. Vesicle Origami: Cuboid Phospholipid Vesicles Formed by Template-Free Self-Assembly. Angew. Chem. Int. Ed. 2017, 56, 6515-6518. [CrossRef] [PubMed]

186. Lasic, D.D. Liposomes: From Physics to Applications; Elsevier: Amsterdam, The Netherlands, 1993.

187. Lasch, J.; Weissig, V.; Brandl, M. Preparation of liposomes. In Liposomes. A Practical Approach, 2nd ed.; Torchilin, V., Weissig, V., Eds.; Oxford University Press: Oxford, UK, 2003; Chapter 1; pp. 3-29.

188. Szoka, F., Jr.; Papahadjopoulos, D. Comparative Properties and Methods of Preparation of Lipid Vesicles (Liposomes). Annu. Rev. Biophys. Bioeng. 1980, 9, 467-508. [CrossRef] [PubMed]

189. Walde, P.; Ichikawa, S. Enzymes inside lipid vesicles: Preparation, reactivity and applications. Biomol. Eng. 2001, 18, 143-177. [CrossRef]

190. Jesorska, A.; Orwar, P. Liposomes: Technologies and Analytical Applications. Annu. Rev. Anal. Chem. 2008, 1, 801-832. [CrossRef] [PubMed]

191. Walde, P. Preparation of Vesicles (Liposomes). In Encyclopedia of Nanoscience and Nanotechnology; Nalwa, H.S., Ed.; American Scientific Publishers: Stevenson Ranch, CA, USA, 2004; Volume 9, pp. 43-79.

192. Walde, P.; Cosentino, K.; Engel, H.; Stano, P. Giant Vesicles: Preparations and Applications. ChemBioChem 2010, 11, 848-865. [CrossRef] [PubMed]

193. Has, C.; Sunthar, P. A comprehensive review on recent preparation techniques of liposomes. J. Liposome Res. 2020, 30, 336-365. [CrossRef] [PubMed]

194. Dimova, R.; Stano, P.; Marques, C.M.; Walde, P. Preparation methods for giant unilamellar vesicles. In The Giant Vesicle Book; Dimova, R., Marques, C.M., Eds.; CRC Press, Taylor \& Francis Group: Boca Raton, FL, USA, 2020; Chapter 1; pp. 3-20.

195. Laouini, A.; Jaafar-Maalej, C.; Limayem-Blouza, I.; Sfar, S.; Charcosset, C.; Fessi, H. Preparation, Characterization and Applications of Liposomes: State of the Art. J. Colloid Sci. Biotechnol. 2012, 1, 147-168. [CrossRef]

196. Olson, F.; Hunt, C.A.; Szoka, F.C.; Vail, J.J.; Papahadjopoulos, D. Preparation of Liposomes of Defined Size Distribution by Extrusion Through Polycarbonate Membranes. Biochim. Biophys. Acta 1979, 557, 9-23. [CrossRef]

197. Mayer, L.D.; Hope, M.J.; Cullis, P.R. Vesicles of variable sizes produced by a rapid extrusion procedure. Biochim. Biophys. Acta 1986, 858, 161-168. [CrossRef]

198. Cullis, P.R.; Mayer, L.D.; Bally, M.B.; Madden, T.D.; Hope, M.J. Generating and loading of liposomal systems for drug-delivery applications. Adv. Drug Deliv. Rev. 1989, 3, 267-282. [CrossRef]

199. MacDonald, R.C.; MacDonald, R.I.; Menco, B.P.M.; Takeshita, K.; Subbarao, N.K.; Hu, L.-R. Small-volume extrusion apparatus for preparation of large, unilamellar vesicles. Biochim. Biophys. Acta 1991, 1061, 297-303. [CrossRef]

200. Hinna, A.; Steiniger, F.; Hupfeld, S.; Stein, P.; Kuntsche, J.; Brandl, M. Filter-extruded liposomes revisited: A study into size distributions and morphologies in relation to lipid-composition and process parameters. J. Liposome Res. 2016, 26, 11-20. [CrossRef] [PubMed]

201. Jousma, H.; Talsma, H.; Spies, F.; Joosten, J.G.H.; Junginger, H.E.; Crommelin, D.J.A. Characterization of liposomes. The influence of extrusion of multilamellar vesicles through polycarbonate membranes on particle size, particle size distribution and number of bilayers. Int. J. Pharm. 1987, 35, 263-274. [CrossRef]

202. Hunter, D.G.; Frisken, B.J. Effect of Extrusion Pressure and Lipid Properties on the Size and Polydispersity of Lipid Vesicles. Biophys. J. 1998, 74, 2996-3002. [CrossRef]

203. Bertrand, M.; Joós, B. Extrusion of small vesicles through nanochannels: A model for experiments and molecular dynamics simulations. Phys. Rev. E 2012, 85, 051910. [CrossRef] [PubMed]

204. Mui, B.; Chow, L.; Hope, M.J. Extrusion Technique to Generate Liposomes of Defined Size. Methods Enzymol. 2003, 367, 3-14. [PubMed]

205. Scott, H.L.; Skinkle, A.; Kelley, E.G.; Waxham, M.N.; Levental, I.; Heberle, F.A. On the Mechanism of Bilayer Separation by Extrusion, or Why Your LUVs Are Not Really Unilamellar. Biophys. J. 2019, 117, 1381-1386. [CrossRef]

206. Huang, C.-H. Studies on Phosphatidylcholine Vesicles. Formation and Physical Characteristics. Biochemistry 1969, 8, 344-352. [CrossRef] [PubMed] 
207. Helfrich, W. The size of bilayer Vesicles generated by sonication. Phys. Lett. 1974, 50A, 115-116. [CrossRef]

208. Szoka, F., Jr.; Papahadjopoulos, D. Procedure for preparation of liposomes with large internal aqueous space and high capture by reverse-phase evaportion. Proc. Natl. Acad. Sci. USA 1978, 75, 4194-4198. [CrossRef] [PubMed]

209. Zumbuehl, O.; Weder, H.-G. Liposomes of controllable size in the range of 40 to $180 \mathrm{~nm}$ by defined dialysis of lipid/detergent mixed micelles. Biochim. Biophys. Acta 1981, 640, 252-262. [CrossRef]

210. Ueno, M.; Tanford, C.; Reynolds, J.A. Phospholipid Vesicle Formation Using Nonionic Detergents with Low Monomer Solubility. Kinetic Factors Determine Vesicle Size and Permeability. Biochemistry 1984, 23, 3070-3076. [CrossRef]

211. Schubert, R. Liposome Preparation by Detergent Removal. Methods Enzymol. 2003, 367, 46-70.

212. Batzri, S.; Korn, E.D. Single bilayer liposomes prepared without sonication. Biochim. Biophys. Acta 1973, 298, 1015-1019. [CrossRef]

213. Kremer, J.M.H.; Van der Esker, M.W.J.; Pathmamanoharan, C.; Wiersema, P.H. Vesicles of Variable Diameter Prepared by a Modified Injection Method. Biochemistry 1977, 16, 3932-3935. [CrossRef] [PubMed]

214. Pons, M.; Foradada, M.; Estelrich, J. Liposomes obtained by the ethanol injection method. Int. J. Pharm. 1993, 95, 51-56. [CrossRef]

215. Wagner, A.; Vorauer-Uhl, K.; Kreismayr, G.; Katinger, H. The crossflow injection technique: An improvement of the ethanol injection method. J. Liposome Res. 2002, 12, 259-270. [CrossRef] [PubMed]

216. Jaafar-Maalej, C.; Diab, R.; Andrieu, V.; Elaissari, A.; Fessi, H. Ethanol injection method for hydrophilic and lipophilic drug-loaded liposome preparation. J. Liposome Res. 2010, 20, 228-243. [CrossRef] [PubMed]

217. Gouda, A.; Sakr, O.S.; Nasr, M.; Sammour, O. Ethanol injection technique for liposome formulation: An insight into development, influencing factors, challenges and applications. J. Drug Deliv. Sci. Technol. 2021, 61, 102174. [CrossRef]

218. Kawamura, J.; Kitamura, H.; Otake, Y.; Fuse, S.; Nakamura, H. Size-Controllable and Scalable Production of Liposomes Using a V-Shaped Mixer Micro-Flow Reactor. Org. Process Res. Dev. 2020, 24, 2122-2127. [CrossRef]

219. Reeves, J.P.; Dowben, R.M. Formation and Properties of Thin-walled Phospholipid Vesicles. J. Cell. Physiol. 1969, 73, 49-60. [CrossRef] [PubMed]

220. Tsumoto, K.; Matsuo, H.; Tomita, M.; Yoshimura, T. Efficient formation of giant liposomes through the gentle hydration of phosphatidylcholine films doped with sugar. Colloids Surf. B 2009, 68, 98-105. [CrossRef] [PubMed]

221. Angelova, M.I.; Dimitrov, D.S. Liposome Electroformation. Faraday Discuss. Chem. Soc. 1986, 81, 303-311. [CrossRef]

222. Angelova, M.I. Liposome Electroformation. In Giant Vesicles; Luisi, P.L., Walde, P., Eds.; Perspectives in Supramolecular Chemistry; John Wiley \& Sons, Ltd.: Chichester, UK, 2000; Volume 6, Chapter 3; pp. $27-43$.

223. Méléard, P.; Bagatolli, L.A.; Pott, T. Giant Unilamellar Vesicle Electroformation: From Lipid Mixtures to Native Membranes Under Physiological Conditions. Methods Enzymol. 2009, 465, 161-175. [PubMed]

224. Shimanouchi, T.; Umakoshi, H.; Kuboi, R. Kinetic Study on Giant Vesicle Formation with Electroformation Method. Langmuir 2009, 25, 4835-4840. [CrossRef]

225. Okumura, Y.; Shuuhei, O. Effect of Counter Electrode in Electroformation of Giant Vesicles. Membranes 2011, 1, 345-353. [CrossRef]

226. Li, Q.; Wang, X.; Ma, S.; Zhang, Y.; Han, X. Electroformation of giant unilamellar vesicles in saline solution. Colloids Surf. B 2016, 147, 368-375. [CrossRef] [PubMed]

227. Drabik, D.; Doskocz, J.; Przybyło, M. Effects of electroformation protocol parameters on quality of homogeneous GUV populations. Chem. Phys. Lipids 2018, 212, 88-95. [CrossRef] [PubMed]

228. Pautot, S.; Frisken, B.J.; Weitz, D.A. Engineering asymmetric vesicles. Proc. Natl. Acad. Sci. USA 2003, 100, 10718-10721. [CrossRef] [PubMed]

229. Carrara, P.; Stano, P.; Luisi, P.L. Giant Vesicles “Colonies": A Model for Primitive Cell Communities. ChemBioChem 2012, 13, 1497-1502. [CrossRef] [PubMed]

230. Moga, A.; Yandrapalli, N.; Dimova, R.; Robinson, T. Optimization of the Inverted Emulsion Method for High-Yield Production of Biomimetic Giant Unilamellar Vesicles. ChemBioChem 2019, 20, 2674-2682. [CrossRef]

231. Xu, B.; Ding, J.; Xu, J.; Yomo, T. Giant Vesicles Produced with Phosphatidylcholines (PCs) and Phosphatidylethanolamines (PEs) by Water-in-Oil Inverted Emulsions. Life 2021, 11, 233. [CrossRef]

232. Sugiura, S.; Kuroiwa, T.; Kagota, T.; Nakajima, M.; Sato, S.; Mukataka, S.; Walde, P.; Ichikawa, S. Novel Method for Obtaining Giant Vesicles from a Monodisperse Water-in-Oil Emulsion Prepared with a Microfluidic Device. Langmuir 2008, 24, 4581-4588. [CrossRef] [PubMed]

233. Funakoshi, K.; Suzuki, H.; Takeuchi, S. Formation of Giant Lipid Vesiclelike Compartments from a Planar Lipid Membrane by a Pulsed Jet Flow. J. Am. Chem. Soc. 2007, 129, 12608-12609. [CrossRef] [PubMed]

234. Stachowiak, J.C.; Richmond, D.L.; Li, T.H.; Liu, A.P.; Parekh, S.H.; Flecther, D.A. Unilamellar vesicle formation and encapsulation by microfluidic jetting. Proc. Natl. Acad. Sci. USA 2008, 105, 4697-4702. [CrossRef] [PubMed]

235. Kamiya, K.; Kawano, R.; Osaki, T.; Akiysohi, K.; Takeuchi, S. Cell-sized asymmetric lipid vesicles facilitate the investigation of asymmetric membranes. Nat. Chem. 2016, 8, 881-889. [CrossRef] [PubMed]

236. Naeff, R. Feasibility of topical liposome drugs produced on an industrial scale. Adv. Drug Deliv. Rev. 1996, 18, 343-347. [CrossRef]

237. Kriftner, R.W. Liposome Production: The Ethanol Injection Technique and the Development of the First Approved Liposome Dermatics. In Liposome Dermatics; Braun-Falco, O., Korting, H.C., Maibach, H.I., Eds.; Springer: Berlin, Germany, $1992 ;$ pp. 91-100.

238. Jahn, A.; Vreeland, W.N.; DeVoe, D.L.; Locascio, L.E.; Gaitan, M. Microfluidic Directed Formation of Liposomes of Controlled Size. Langmuir 2007, 23, 6289-6293. [CrossRef] [PubMed] 
239. Jahn, A.; Stavis, S.M.; Hong, J.S.; Vreeland, W.N.; DeVoe, D.L.; Gaitan, M. Microfluidic Mixing and the Formation of Nanoscale Lipid Vesicles. ACS Nano 2010, 4, 2077-2087. [CrossRef] [PubMed]

240. Jahn, A.; Lucas, F.; Wepf, R.A.; Dittrich, P.S. Freezing Continuous-Flow Self-Assembly in a Microfluidic Device: Toward Imaging of Liposome Formation. Langmuir 2013, 29, 1717-1723. [CrossRef]

241. Bottaro, E.; Nastruzzi, C. "Off-the-shelf” microfluidic devices for the production of liposomes for drug delivery. Mater. Sci. Eng. C 2016, 64, 29-33. [CrossRef] [PubMed]

242. Malouff, T.D.; Seneviratne, D.S.; Ebner, D.K.; Stross, W.C.; Waddle, M.R.; Trifiletti, D.M.; Krishnan, S. Boron Neutron Capture Therapy: A Review of Clinical Applications. Front. Oncol. 2021, 11, 601820. [CrossRef] [PubMed]

243. Costa, C.; Liu, Z.; Simões, S.L.; Correia, A.; Rahikkala, A.; Seitsonen, J.; Ruokolainen, J.; Aguiar-Ricardo, A.; Santos, H.A.; Corvo, M.L. One-step microfluidics production of enzyme-loaded liposomes for the treatment of inflammatory diseases. Colloids Surf. B 2021, 199, 111556. [CrossRef] [PubMed]

244. Keele, B.B., Jr.; McCord, J.M.; Fridovich, I. Further Characterization of Bovine Superoxide Dismutase and Its Isolation from Bovine Heart. J. Biol. Chem. 1971, 246, 2875-2880. [CrossRef]

245. Hyslop, P.A.; Morel, B.; Sauerheber, R.D. Organization and Interaction of Cholesterol and Phosphatidylcholine in Model Bilayer Membranes. Biochemistry 1990, 29, 1025-1039. [CrossRef] [PubMed]

246. Patil, Y.P.; Jadhav, S. Novel methods for liposome preparation. Chem. Phys. Lipids 2014, 177, 8-18. [CrossRef] [PubMed]

247. Santo, I.E.; Campardelli, R.; Albuquerque, E.C.; de Melo, S.V.; Della Porta, G.; Reverchon, E. Liposomes preparation using a supercritical fluid assisted continuous process. Chem. Eng. J. 2014, 249, 153-159. [CrossRef]

248. Campardalli, R.; Trucillo, P.; Reverchon, E. A Supercritical Fluid-Based Process for the Production of Fluorescein-Loaded Liposomes. Ind. Eng. Chem. Res. 2016, 55, 5359-5365. [CrossRef]

249. Bigazzi, W.; Penoy, N.; Evrard, B.; Piel, G. Supercritical fluid methods: An alternative to conventional methods to prepare liposomes. Chem. Eng. J. 2020, 383, 123106.

250. Khadke, S.; Roces, C.B.; Donaghey, R.; Giacobbo, V.; Su, Y.; Perrie, Y. Scalable solvent-free production of liposomes. J. Pharm. Pharmacol. 2020, 72, 1328-1340. [CrossRef] [PubMed]

251. Leitgeb, M.; Knez, Ž.; Primožič, M. Sustainable technologies for liposome preparation. J. Supercrit. Fluids 2020, $165,104984$.

252. Trucillo, P.; Reverchon, E. Production of PEG-coated liposomes using a continuous supercritical assisted process. J. Supercrit. Fluids 2021, 167, 105048. [CrossRef]

253. Zhigaltsev, I.V.; Tam, Y.K.; Leung, A.K.K.; Cullis, P.R. Production of limit size nanoliposomal systems with potential utility as ultra-small drug delivery agents. J. Liposome Res. 2016, 26, 96-102. [CrossRef] [PubMed]

254. Ota, S.; Yoshizawa, S.; Takeuchi, S. Microfluidic Formation of Monodisperse, Cell-Sized, and Unilamellar Vesicles. Angew. Chem. Int. Ed. 2009, 48, 6533-6537. [CrossRef] [PubMed]

255. Teh, S.-Y.; Khnouf, R.; Fan, H.; Lee, A.P. Stable, biocompatible lipid vesicle generation by solvent extraction-based droplet microfluidics. Biomicrofluidics 2011, 5, 044113. [CrossRef]

256. Matosevic, S.; Paegel, B.M. Stepwise Synthesis of Giant Unilamellar Vesicles on a Microfluidic Assembly Line. J. Am. Chem. Soc. 2011, 133, 2798-2800. [CrossRef] [PubMed]

257. van Swaay, D.; deMello, A. Microfluidic methods for forming liposomes. Lab Chip 2013, 13, 752-767. [CrossRef]

258. Carugo, D.; Bottaro, E.; Owen, J.; Stride, E.; Nastruzzi, C. Liposome production by microfluidics: Potential and limiting factors. Sci. Rep. 2016, 6, 25876. [CrossRef] [PubMed]

259. Deshpande, S.; Caspi, Y.; Meijering, A.E.C.; Dekker, C. Octanol-assisted liposome assembly on chip. Nat. Commun. 2016, 7, 10447. [CrossRef] [PubMed]

260. Deshpande, S.; Dekker, C. On-chip microfluidic production of cell-sized liposomes. Nat. Protoc. 2018, 13, 856-874. [CrossRef]

261. Robinson, T. Microfluidics and giant vesicles: Creation, capture, and applications for biomembranes. In Multiresponsive Behavior of Biomembranes and Giant Vesicles. Biomembranes and Lipid Self-Assembly; Academic Press: London, UK, 2019; Volume 30, Chapter 3; pp. 271-315.

262. Yandrapalli, N.; Seemann, T.; Robinson, T. On-Chip Inverted Emulsion Method for Fast Giant Vesicle Production, Handling, and Analysis. Micromachines 2020, 11, 285. [CrossRef] [PubMed]

263. Schaich, M.; Sobota, D.; Sleath, H.; Cam, J.; Keyser, U.F. Characterization of lipid composition and diffusivity in OLA generated vesicles. Biochim. Biophys. Acta, Biomembr. 2020, 1862, 183359. [CrossRef]

264. Litschel, T.; Schwille, P. Protein Reconstitution Inside Giant Unilamellar Vesicles. Annu. Rev. Biophys. 2021, 50, 525-548. [CrossRef] [PubMed]

265. Kirchner, S.R.; Ohlinger, A.; Pfeiffer, T.; Urban, A.S.; Stefani, F.D.; Deak, A.; Lutich, A.A.; Feldmann, J. Membrane composition of jetted lipid vesicles: A Raman spectroscopy study. J. Biophotonics 2012, 5, 40-46. [CrossRef] [PubMed]

266. New, R.R.C. (Ed.) Characterization of liposomes. In Liposomes. A practical Approach; Oxford University Press: Oxford, UK, 1990; Chapter 3; pp. 105-161.

267. Zuidam, N.J.; de Vruh, R.; Crommelin, D.J.A. Characterization of liposomes. In Liposomes. A Practical Approach, 2nd ed.; Torchilin, V., Weissig, V., Eds.; Oxford University Press: Oxford, UK, 2003; Chapter 2; pp. 31-78.

268. London, E. Membrane Structure-Function Insights from Asymmetric Lipid Vesicles. Acc. Chem. Res. 2019, 52, $2382-2391$. [CrossRef] [PubMed] 
269. Wimley, W.C.; Thompson, T.E. Exchange and Flip-Flop of Dimyristoylphosphatidylcholine in Liquid-Crystalline, Gel, and Two-Component, Two-Phase Large Unilamellar Vesicles. Biochemistry 1990, 29, 1296-1303. [CrossRef]

270. Gurtovenko, A.A.; Vattulainen, I. Molecular Mechanism for Lipid Flip-Flop. J. Phys. Chem. B 2007, 111, 13554-13559. [CrossRef]

271. John, K.; Schreiber, S.; Kubelt, J.; Herrmann, A.; Müller, P. Transbilayer Movement of Phospholipids at the Main Phase Transition of Lipid Membranes: Implications for Rapid Flip. Biophys. J. 2002, 83, 3315-3323. [CrossRef]

272. Marquardt, D.; Heberle, F.A.; Miti, T.; Eicher, B.; London, E.; Katsaras, J.; Pabst, G. 1H NMR Shows Phospholipid Flip-Flop in Gel and Fluid Bilayers. Langmuir 2017, 33, 3731-3741. [CrossRef] [PubMed]

273. Hope, M.J.; Redelmeier, T.E.; Wong, K.F.; Rodrigueza, W.; Cullis, P.R. Phospholipid Asymmetry in Large Unilamellar Vesicles Induced by Transmembrane pH Gradients. Biochemistry 1989, 28, 4181-4187. [CrossRef] [PubMed]

274. Cheng, H.-T.; Megha; London, E. Preparation and Properties of Asymmetric Vesicles That Mimic Cell Membranes. Effect upon Lipid Raft Formation and Transmembrane Helix Orientation. J. Biol. Chem. 2009, 284, 6079-6092. [CrossRef] [PubMed]

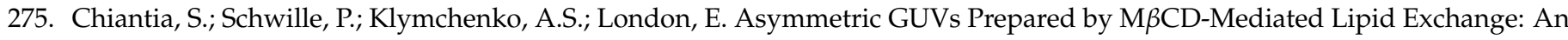
FCS Study. Biophys. J. 2011, 100, L01-L03. [CrossRef]

276. Hu, P.C.; Li, S.; Malmstadt, N. Microfluidic Fabrication of Asymmetric Giant Lipid Vesicles. ACS Appl. Mater. Interfaces 2011, 3 , 1434-1440. [CrossRef] [PubMed]

277. Doktorova, M.; Heberle, F.A.; Eicher, B.; Standaert, R.F.; Katsaras, J.; London, E.; Pabst, G.; Marquardt, D. Preparation of asymmetric phospholipid vesicles for use as cell membrane models. Nat. Protoc. 2018, 13, 2086-2101. [CrossRef]

278. Markones, M.; Drechsler, C.; Kaiser, M.; Kalie, L.; Heerklotz, H.; Fiedler, S. Engineering Asymmetric Lipid Vesicles: Accurate and Convenient Control of the Outer Leaflet Lipid Composition. Langmuir 2018, 34, 1999-2005. [CrossRef]

279. Li, B.; London, E. Preparation and Drug Entrapment Properties of Asymmetric Liposomes Containing Cationic and Anionic Lipids. Langmuir 2020, 36, 12521-12531. [CrossRef] [PubMed]

280. Kisak, E.T.; Coldren, B.; Zasadzinski, J.A. Nanocompartments Enclosing Vesicles, Colloids, and Macromolecules via Interdigitated Lipid Bilayers. Langmuir 2002, 18, 284-288. [CrossRef]

281. Giuliano, C.B.; Cvjetan, N.; Ayache, J.; Walde, P. Multivesicular Vesicles: Preparation and Applications. ChemSystemsChem 2021, 3, e2000049.

282. Sunamoto, J.; Sato, T.; Hirota, M.; Fukushima, K.; Hiratani, K.; Hara, K. A newly developed immunoliposome-An egg phosphatidylcholine liposome coated with pullulan bearing both a cholesterol moiety and an IgMs fragment. Biochim. Biophys. Acta 1987, 898, 323-330. [CrossRef]

283. Mastrobattista, E.; Koning, G.A.; Storm, G. Immunoliposomes for the targeted delivery of antitumor drugs. Adv. Drug Deliv. Rev. 1999, 40, 103-127. [CrossRef]

284. Maruyama, K. PEG-Immunoliposome. Biosci. Rep. 2002, 22, 251-266. [CrossRef]

285. Eloy, J.O.; Petrilli, R.; Trevizan, L.N.F.; Chorilli, M. Immunoliposomes: A review on functionalization strategies and targets for drug delivery. Colloids Surf. B 2017, 159, 454-467. [CrossRef] [PubMed]

286. Merino, M.; Zalba, S.; Garrido, M.J. Immunoliposomes in clinical oncology: State of the art and future perspective. J. Control. Release 2018, 275, 162-176. [CrossRef] [PubMed]

287. Beck, P.; Liebi, M.; Kohlbrecher, J.; Ishikawa, T.; Rüegger, H.; Fischer, P.; Walde, P.; Windhab, E. Novel Type of Bicellar Disks from a Mixture of DMPC and DMPE-DTPA with Complexed Lanthanides. Langmuir 2010, 26, 5382-5387. [CrossRef] [PubMed]

288. Liebi, M. Tailored Phospholipid Bicelles to Generate Magnetically Switchable Material. Ph.D. Thesis, ETH Zürich, Zürich, Switzerland, 2013. (no. 21048).

289. Beck, P.; Liebi, M.; Kohlbrecher, J.; Ishikawa, T.; Rüegger, H.; Zepik, H.; Fischer, P.; Walde, P.; Windhab, E. Magnetic Field Alignable Domains in Phospholipid Vesicle Membranes Containing Lanthanides. J. Phys. Chem. B 2010, 114, 174-186. [CrossRef]

290. Beck, P. Magnetic Field Assisted Biomaterials Processing. Ph.D. Thesis, ETH Zürich, Zürich, Switzerland, 2009. (no. 18292).

291. Isabettini, S.; Massabni, S.; Kohlbrecher, J.; Schuler, L.D.; Walde, P.; Sturm, M.; Windhab, E.J.; Fischer, P.; Kuster, S. Understanding the Enhanced Magnetic Response of Aminocholesterol Doped Lanthanide-Ion-Chelating Phospholipid Bicelles. Langmuir 2017, 33, 8533-8544. [CrossRef] [PubMed]

292. Sanders, C.R.; Schwonek, J.P. Characterization of Magnetically Orientable Bilayers in Mixtures of Dihexanoylphosphatidycholine and Dimyristoylphosphatidylcholine by Solid-State NMR. Biochemistry 1992, 31, 8898-8905. [CrossRef]

293. Luchette, P.A.; Vetman, T.N.; Prosser, R.S.; Hancock, R.E.W.; Nieh, M.-P.; Glinka, C.J.; Krueger, S.; Katsaras, J. Morphology of fast-tumbling bicelles: A small angle neutron scattering and NMR study. Biochim. Biophys. Acta 2001, 1513, 83-94. [CrossRef]

294. Triba, M.N.; Warschawski, D.E.; Devaux, P.F. Reinvestigation by Phosphorous NMR of Lipid Distribution in Bicelles. Biophys. J. 2005, 88, 1887-1901. [CrossRef]

295. Suga, K.; Kitagawa, K.; Taguchi, S.; Okamoto, Y.; Umkoshi, H. Evaluation of Molecular Ordering in Bicelle Bilayer Membranes Based on Induced Circular Dichroism Spectra. Langmuir 2020, 36, 3242-3250. [CrossRef] [PubMed]

296. Veatch, S.L.; Keller, S.L. Separation of Liquid Phases in Giant Vesicles of Ternary Mixtures of Phospholipids and Cholesterol. Biophys. J. 2003, 85, 3074-3083. [CrossRef]

297. de Almeida, R.F.M.; Fedorov, A.; Prieto, M. Sphingomyelin/Phosphatidylcholine/Cholesterol Phase Diagram: Boundaries and Composition of Lipid Rafts. Biophys. J. 2003, 85, 2406-2416. [CrossRef]

298. Veatch, S.L.; Polozov, I.V.; Gawrisch, K.; Keller, S.L. Liquid Domains in Vesicles Investigated by NMR and Fluorescence Microscopy. Biophys. J. 2004, 86, 2910-2922. [CrossRef] 
299. Polozov, I.V.; Gawrisch, K. Characterization of the Liquid-Ordered State by Proton MAS NMR. Biophys. J. 2006, 90, $2051-2061$. [CrossRef] [PubMed]

300. M’Baye, G.; Mély, Y.; Duportail, G.; Klymchenko, A.S. Liquid Ordered and Gel Phases of Lipid Bilayers: Fluorescent Probes Reveal Close Fluidity but Different Hydration. Biophys. J. 2008, 95, 1217-1225. [CrossRef] [PubMed]

301. Feigenson, G.W. Phase diagrams and lipid domains in multicomponent lipid bilayer mixtures. Biochim. Biophys. Acta 2009, 1788, 47-52. [CrossRef]

302. Honerkamp-Smith, A.R.; Veatch, S.L.; Keller, S.L. An introduction to critical points for biophysicists; observations of compositional heterogeneity in lipid membranes. Biochim. Biophys. Acta 2009, 1788, 53-63. [CrossRef] [PubMed]

303. Konyakhina, T.M.; Wu, J.; Mastroianni, J.D.; Heberle, F.A.; Feigenson, G.W. Phase diagram of a 4-component lipid mixture: DSPC/DOPC/POPC/chol. Biochim. Biophys. Acta 2013, 1828, 2204-2214. [CrossRef]

304. Suga, K.; Umakoshi, H. Detection of Nanosized Ordered Domains in DOPC/DPPC and DOPC/Ch Binary Lipid Mixture Systems of Large Unilamellar Vesicles Using TEMPO Quenching Method. Langmuir 2013, 29, 4830-4838. [CrossRef] [PubMed]

305. Rheinstädter, M.C.; Mouritsen, O.G. Small-scale structure in fluid cholesterol-lipid bilayers. Curr. Opin. Colloid Interface Sci. 2013, 18, 440-447. [CrossRef]

306. Klymchenko, A.S.; Kreder, R. Fluorescent Probes for Lipid Rafts: From Model Membranes to Living Cells. Chem. Biol. 2014, 21, 97-113. [CrossRef]

307. Feigenson, G.W. Pictures of the Substructure of Liquid-Ordered Domains. Biophys. J. 2015, 109, 854-855. [CrossRef] [PubMed]

308. Engberg, O.; Hautala, V.; Yasuda, T.; Dehio, H.; Murata, M.; Slotte, J.P.; Nyholm, T.K.M. The Affinity of Cholesterol for Different Phospholipids Affects Lateral Segregation in Bilayers. Biophys. J. 2016, 111, 546-556. [CrossRef] [PubMed]

309. Cebecauer, M.; Amaro, M.; Jurkiewicz, P.; Sarmento, M.J.; Šachl, R.; Cwiklik, L.; Hof, M. Membrane Lipid Nanodomains. Chem. Rev. 2018, 118, 11259-11297. [CrossRef] [PubMed]

310. Bolmatov, D.; Soloviov, D.; Zhernenkov, M.; Zav'yalov, D.; Mamontov, E.; Suvorov, A.; Cai, Y.Q.; Katsaras, J. Molecular picture of the transient nature of lipid rafts. Langmuir 2020, 36, 4887-4896. [CrossRef]

311. Cornell, C.E.; Mileant, A.; Thakkar, N.; Lee, K.K.; Keller, S.L. Direct imaging of liquid domains in membranes by cryo-electron tomography. Proc. Natl. Acad. Sci. USA 2020, 117, 19713-19719. [CrossRef] [PubMed]

312. Blosser, M.C.; Cornell, C.E.; Rayermann, S.P.; Keller, S.L. Phase diagrams and tie lines in giant unilamellar vesicles. In The Giant Vesicle Book; Dimova, R., Marques, C.M., Eds.; CRC Press, Taylor \& Francis Group: Boca Raton, FL, USA, 2020; Chapter 18; pp. 401-416.

313. Feigenson, G.W. Phase behavior of lipid mixtures. Nat. Chem. Biol. 2006, 2, 560-563. [CrossRef] [PubMed]

314. Walde, P.; Blöchliger, E. Circular Dichroic Properties of Phosphatidylcholine Liposomes. Langmuir 1997, 13, 1668-1671. [CrossRef]

315. Wagner, A.; Vorauer-Uhl, K.; Kreismayr, G.; Katinger, H. Enhanced protein loading into liposomes by the multiple crossflow injection technique. J. Liposome Res. 2002, 12, 271-283. [CrossRef] [PubMed]

316. Kuroiwa, T.; Fujita, R.; Kobayashi, I.; Uemura, K.; Nakajima, M.; Sato, S.; Walde, P.; Ichikawa, S. Efficient Preparation of Giant Vesicles as Biomimetic Compartment Systems with High Entrapment Yields for Biomacromolecules. Chem. Biodiv. 2012, 9, 2453-2472. [CrossRef] [PubMed]

317. Deamer, D.W.; Barchfeld, G.L. Encapsulation of Macromolecules by Lipid Vesicles under Simulated Prebiotic Conditions. J. Mol. Evol. 1982, 18, 203-206. [CrossRef]

318. Kirby, C.; Gregoriadis, G. Dehydration-Rehydration Vesicles: A Simple Method for High Yield Drug Entrapment in Liposomes. Bio/Technology 1984, 2, 979-984. [CrossRef]

319. Fenske, D.B.; Cullis, P.R. Medical Applications of Lipid Nanoparticles. In Liposomes, Lipid Bilayers and Model Membranes. From Basic Research to Applications; Pabst, G., Kučerka, N., Nieh, M.-P., Katsaras, J., Eds.; CRC Press, Taylor \& Francis Group: Boca Raton, FL, USA, 2014; Chapter 15; pp. 291-315.

320. Mayer, L.D.; Bally, M.B.; Cullis, P.R. Uptake of adriamycin into large unilamellar vesicles in response to a pH gradient. Biochim. Biophys. Acta 1986, 857, 123-126. [CrossRef]

321. Haran, G.; Cohen, R.; Bar, L.K.; Barenholz, Y. Transmembrane ammonium sulfate gradients in liposomes produce efficient and stable entrapment of amphipathic weak bases. Biochim. Biophys. Acta 1993, 1151, 201-215. [CrossRef]

322. Barenholz, Y. Liposome applications: Problems and prospects. Curr. Opin. Colloid Interface Sci. 2001, 6, 66-77. [CrossRef]

323. Abraham, S.A.; Waterhouse, D.N.; Mayer, L.D.; Cullis, P.R.; Madden, T.D.; Bally, M.B. The Liposomal Formulation of Doxorubicin. Methods Enzymol. 2005, 391, 71-97. [PubMed]

324. Barenholz, Y. Doxil ${ }^{\circledR}$ —The first FDA-approved nano-drug: Lessons learned. J. Control. Release 2012, 160, 117-134. [CrossRef] [PubMed]

325. Zucker, D.; Marcus, D.; Barenholz, Y.; Goldblum, A. Liposome drugs' loading efficiency: A working model based on loading conditions and drug's physicochemical properties. J. Control. Release 2009, 139, 73-80. [CrossRef]

326. Gubernator, J. Active methods of drug loading into liposomes: Recent strategies for stable drug entrapment and increased in vivo activity. Expert Opin. Drug Deliv. 2011, 8, 565-580. [CrossRef]

327. Li, T.; Cipolla, D.; Rades, T.; Boyd, B.J. Drug nanocrystallisation within liposomes. J. Control. Release 2018, 288, 96-110. [CrossRef] [PubMed]

328. Fritze, A.; Hens, F.; Kimpfler, A.; Schubert, R.; Peschka-Süss, R. Remote loading of doxorubicin into liposomes driven by a transmembrane phosphate gradient. Biochim. Biophys. Acta 2006, 1758, 1633-1640. [CrossRef] 
329. Schilt, Y.; Berman, T.; Wei, X.; Barenholz, Y.; Raviv, U. Using solution X-ray scattering to determine the high-resolution structure and morphology of PEGylated liposomal doxorubicin nanodrugs. Biochim. Biophys. Acta 2016, 1860, 108-119. [CrossRef]

330. Lasic, D.D. Applications of Liposomes. In Structure and Dynamics of Membranes: From Cells to Vesicles; Lipowsky, R., Sackmann, E., Eds.; Handbook of Biological Physics; Elsevier Science B.V.: Amsterdam, The Netherlands, 1995; Volume 1A, Chapter 10; pp. 491-519.

331. Martí, M.; de la Maza, A.; Parra, J.L.; Coderch, L. Role of Liposomes in Textile Dyeing. In Liposomes, Lipid Bilayers and Model Membranes. From Basic Research to Applications; Pabst, G., Kučerka, N., Nieh, M.-P., Katsaras, J., Eds.; CRC Press, Taylor \& Francis Group: Boca Raton, FL, USA, 2014; Chapter 20; pp. 401-414.

332. Bulbake, U.; Doppalapudi, S.; Kommineni, N.; Khan, W. Liposomal Formulations in Clinical Use: An Updated Review. Pharmaceutics 2017, 9, 12. [CrossRef]

333. Kapoor, B.; Gupta, R.; Gulati, M.; Singh, S.K.; Khursheed, R.; Gupta, M. The Why, Where, Who, How, and What of the vesicular delivery systems. Adv. Colloid Interface Sci. 2019, 271, 101985. [CrossRef] [PubMed]

334. Beltrán-Gracia, E.; López-Camacho, A.; Higuera-Ciapara, I.; Velázquez-Fernández, J.B.; Vallejo-Cardona, A.A. Nanomedicine review: Clinical developments in liposomal applications. Cancer Nanotechnol. 2019, 10, 11. [CrossRef]

335. Bisso, S.; Leroux, J.-C. Nanopharmaceuticals: A focus on their clinical translatability. Int. J. Pharm. 2020, 578, 119098. [CrossRef] [PubMed]

336. Thi, T.T.H.; Suys, E.J.A.; Lee, J.S.; Nguyen, D.H.; Park, K.D.; Truong, N.P. Lipid-Based Nanoparticles in the Clinic and Clinical Trials: From Cancer Nanomedicine to COVID-19 Vaccines. Vaccines 2021, 9, 359. [CrossRef] [PubMed]

337. Huckriede, A.; Bungener, L.; Stegmann, T.; Daemen, T.; Medema, J.; Palache, A.M.; Wilschut, J. The virosome concept for influenza vaccines. Vaccine 2005, 23, S26-S38. [CrossRef] [PubMed]

338. Herzog, C.; Hartmann, K.; Künzi, V.; Kürsteiner, O.; Mischler, R.; Lazar, H.; Glück, R. Eleven years of Inflexal ${ }^{\circledR}$ V—A virosomal adjuvanted influenza vaccine. Vaccine 2009, 27, 4381-4387. [CrossRef]

339. Schwendener, R.A. Liposomes as vaccine delivery systems: A review of the recent advances. Ther. Adv. Vaccines 2014, 2, 159-182. [CrossRef] [PubMed]

340. De Serrano, L.O.; Burkhart, D.J. Liposomal vaccine formulations as prophylactic agents: Design considerations for modern vaccines. J. Nanobiotechnol. 2017, 15, 83. [CrossRef] [PubMed]

341. Meybeck, A. Past, Present and Future of Liposome Cosmetics. In Liposome Dermatics; Braun-Falco, O., Korting, H.C., Maibach, H.I., Eds.; Springer: Berlin, Germany, 1992; pp. 341-352.

342. Lautenschläger, H. Liposomes. In Handbook of Cosmetic Science and Technology, 2nd ed.; Paye, M., Barel, A.O., Maubach, H.I., Eds.; CRC Press: Boca Raton, FL, USA, 2006; pp. 155-163.

343. Rahimpor, Y.; Hamishehkar, H. Liposomes in cosmeceutics. Expert Opin. Drug Deliv. 2012, 9, 443-455. [CrossRef] [PubMed]

344. van Hoogevest, P.; Fahr, A. Phospholipids in Cosmetic Carriers. In Nanocosmetics. From Ideas to Products; Cornier, J., Keck, C.M., Van de Voorde, M., Eds.; Springer Nature Switzerland AG: Cham, Switzerland, 2019; Chapter 6; pp. 95-140.

345. Chen, C.; Han, D.; Cai, C.; Tang, X. An overview of liposome lyophilization and its future potential. J. Control. Release 2010, 142, 299-311. [CrossRef]

346. Franzé, S.; Selmin, F.; Samaritani, F.; Minghetti, P.; Cilurzo, F. Lyophilization of Liposomal Formulations: Still Necessary, Still Challenging. Pharmaceutics 2018, 10, 139. [CrossRef]

347. Belfiore, L.; Saunders, D.N.; Ranson, M.; Thurecht, K.J.; Storm, G.; Vine, K.L. Towards clinical translation of ligand-functionalized liposomes in targeted cancer therapy: Challenges and opportunities. J. Control. Release 2018, 277, 1-13. [CrossRef] [PubMed]

348. Sackmann, E. Physical Basis of Self-Organization and Function of Membranes: Physics of Vesicles. In Structure and Dynamics of Membranes: From Cells to Vesicles; Lipowsky, R., Sackmann, E., Eds.; Handbook of Biological Physics; Elsevier Science B.V.: Amsterdam, The Netherlands, 1995; Volume 1A, Chapter 5; pp. 213-304.

349. Seifert, U.; Lipowsky, R. Morphology of Vesicles. In Structure and Dynamics of Membranes: From Cells to Vesicles; Lipowsky, R., Sackmann, E., Eds.; Handbook of Biological Physics; Elsevier Science B.V.: Amsterdam, The Netherlands, 1995; Volume 1A, Chapter 8; pp. 403-463.

350. Jimbo, T.; Sakuma, Y.; Urakami, N.; Ziherl, P.; Imai, M. Role of Inverse-Cone-Shape Lipids in Temperature-Controlled SelfReproduction of Binary Vesicles. Biophys. J. 2016, 110, 1551-1562. [CrossRef] [PubMed]

351. Méléard, P.; Gerbeaud, C.; Pott, T.; Fernandez-Puente, L.; Bivas, I.; Mitov, M.D.; Dufourcq, J.; Bothorel, P. Bending Elasticity of Model Membranes: Influences of Temperature and Sterol Content. Biophys. J. 1997, 72, 2616-2629. [CrossRef]

352. Helfrich, W. Bending Elasticity of Fluid Membranes. In Giant Vesicles; Luisi, P.L., Walde, P., Eds.; Perspectives in Supramolecular, Chemistry; John Wiley \& Sons, Ltd.: Chichester, UK, 2000; Volume 6, Chapter 6; pp. 51-70.

353. Döbereiner, H.-G.; Käs, J.; Noppl, D.; Sprenger, I.; Sackmann, E. Budding and Fission of Vesicles. Biophys. J. 1993, 65, 1396-1403. [CrossRef]

354. Morowitz, H.J.; Heinz, B.; Deamer, D.W. The chemical logic of a minimum protocell. Origins Life Evol. Biospheres 1988, 18, 281-287.

355. Svetina, S. Vesicle Budding and the Origin of Cellular Life. ChemPhysChem 2009, 10, 2769-2776. [CrossRef]

356. Damer, B.; Deamer, D. Coupled Phases and Combinatorial Selection in Fluctuating Hydrothermal Pools: A Scenario to Guide Experimental Approaches to the Origin of Cellular Life. Life 2015, 5, 872-887. [CrossRef] [PubMed] 
357. Chen, I.A.; Salehi-Ashtiani, K.; Szostak, J.W. RNA Catalysis in Model Protocell Vesicles. J. Am. Chem. Soc. 2005, 127, 13213-13219. [CrossRef] [PubMed]

358. Mansy, S.S.; Schrum, J.P.; Krishnamurthy, M.; Tobé, S.; Treco, D.A.; Szostak, J.W. Template-directed synthesis of a genetic polymer in a model protocell. Nature 2008, 454, 122-125. [CrossRef] [PubMed]

359. Deamer, D. First Life. Discovering the Connections Between Stars, Cells, and How Life Began; University of California Press: Berkeley/Los Angeles, CA, USA, 2011.

360. Luisi, P.L. The Emergence of Life. From Chemical Origins to Synthetic Biology, 2nd ed.; Cambridge University Press: Cambridge, UK, 2016.

361. Chen, I.A.; Walde, P. From Self-Assembled Vesicles to Protocells. Cold Spring Harb. Perspect. Biol. 2010, 2, a002170. [CrossRef] [PubMed]

362. Monnard, P.-A.; Walde, P. Current Ideas about Prebiological Compartmentalization. Life 2015, 5, 1239-1263. [CrossRef]

363. Lai, Y.-C.; Chen, I.A. Protocells. Curr. Biol. 2020, 30, R484-R485. [CrossRef] [PubMed]

364. Uchegbu, I.F.; Florence, A.T. Non-ionic Surfactant Vesicles (Niosomes): Physical and Pharmaceutical Chemistry. Adv. Colloid Interface Sci. 1995, 58, 1-55. [CrossRef]

365. Bartelds, R.; Nematollahi, M.H.; Pols, T.; Stuart, M.C.A.; Pardakhty, A.; Asadikaram, G.; Poolman, B. Niosomes, an alternative for liposomal delivery. PLoS ONE 2018, 13, e0194179. [CrossRef] [PubMed]

366. Chen, S.; Hanning, S.; Falconer, J.; Locke, M.; Wen, J. Recent advances in non-ionic surfactant vesicles (niosomes): Fabrication, characterization, pharmaceutical and cosmetic applications. Eur. J. Pharm. Biopharm. 2019, 144, 18-39. [CrossRef] [PubMed]

367. Fendler, J.H. Surfactant Vesicles as Membrane Mimetic Agents: Characterization and Utilization. Acc. Chem. Res. 1980, 13, 7-13. [CrossRef]

368. Kunitake, T.; Okahata, Y. Synthetic Bilayer Membranes with Anionic Head Groups. Bull. Chem. Soc. Jpn. 1978, 51, 1877-1879. [CrossRef]

369. Guo, Z.; Hauser, N.; Moreno, A.; Ishikawa, T.; Walde, P. AOT vesicles as templates for the horseradish peroxidase-triggered polymerization of aniline. Soft Matter 2011, 7, 180-193. [CrossRef]

370. Junker, K.; Zandomeneghi, G.; Guo, Z.; Kissner, R.; Ishikawa, T.; Kohlbrecher, J.; Walde, P. Mechanistic aspects of the horseradish peroxidase-catalysed polymerisation of aniline in presence of AOT vesicles as templates. RSC Adv. 2012, 2, 6478-6495. [CrossRef]

371. Gosh, S.; Roy, A.; Banik, D.; Kundu, N.; Kuchlyan, J.; Dhir, A.; Sarkar, N. How Does the Surface Charge of Ionic Surfactant and Cholesterol Forming Vesicles Control Rotational and Translational Motion of Rhodamine 6G Perchlorate $\left(\mathrm{R}_{6} \mathrm{G} \mathrm{ClO} 4\right)$ ? Langmuir 2015, 31, 2310-2320. [CrossRef]

372. Viseu, M.I.; Edwards, K.; Campos, C.S.; Costa, S.M.B. Spontaneous Vesicles Formed in Aqueous Mixtures of Two Cationic Amphiphiles. Langmuir 2000, 16, 2105-2114. [CrossRef]

373. Kunitake, T.; Okahata, Y. A Totally Synthetic Bilayer Membrane. J. Am. Chem. Soc. 1977, 99, 3860-3861. [CrossRef]

374. Sreejith, L.; Parathakkat, S.; Nair, S.M.; Kumar, S.; Varma, G.; Hassan, P.A.; Talmon, Y. Octanol-Triggered Self-Assemblies of the CTAB/KBr System: A Microstructural Study. J. Phys. Chem. B. 2011, 115, 464-470. [CrossRef] [PubMed]

375. Ferrer-Tasies, L.; Moreno-Calvo, E.; Cano-Sarabia, M.; Aguilella-Arzo, M.; Angelova, A.; Lesieur, S.; Ricart, S.; Faraudo, J.; Ventosa, N.; Veciana, J. Quatsomes: Vesicles Formed by Self-Assembly of Sterols and Quaternary Ammonium Surfactants. Langmuir 2013, 29, 6519-6528. [CrossRef] [PubMed]

376. Marques, E.M.; Regev, O.; Khan, A.; Miguel, M.d.G.; Lindman, B. Vesicle Formation and General Phase Behavior in the Catanionic Mixture SDS-DDAB-Water. The Anionic-Rich Side. J. Phys. Chem. B 1998, 102, 6746-6758. [CrossRef]

377. Marques, E.F.; Regev, O.; Khan, A.; Miguel, M.dG.; Lindman, B. Vesicle Formation and General Phase Behavior in the Cationic Mixture SDS-DDAB-Water. The Cationic-Rich Side. J. Phys. Chem. B 1999, 103, 8353-8363. [CrossRef]

378. Kaler, E.W.; Murthy, A.K.; Rodriguez, B.E.; Zasadzinski, J.A.N. Spontaneous Vesicle Formation in Aqueous Mixtures of SingleTailed Surfactants. Science 1989, 245, 1371-1374. [CrossRef] [PubMed]

379. Discher, D.E.; Ahmed, F. Polymersomes. Annu. Rev. Biomed. Eng. 2006, 8, 323-341. [CrossRef] [PubMed]

380. Kita-Tokarczyk, K.; Grumelard, J.; Haefele, T.; Meier, T. Block copolymer vesicles-Using concepts from polymer chemistry to mimic biomembranes. Polymer 2005, 46, 3540-3563. [CrossRef]

381. LoPresti, C.; Lomas, H.; Massignani, M.; Smart, T.; Battaglia, G. Polymersomes: Nature inspired nanometer sized compartments. J. Mater. Chem. 2009, 19, 3576-3590. [CrossRef]

382. Tanner, P.; Baumann, P.; Enea, R.; Onaca, O.; Palivan, C.; Meier, W. Polymeric Vesicles: From Drug Carriers to Nanoreactors and Artificial Organelles. Acc. Chem. Res. 2011, 44, 1039-1049. [CrossRef] [PubMed]

383. Palivan, C.G.; Goers, R.; Najer, A.; Zhang, X.; Car, A.; Meier, W. Bioinspired polymer vesicles and membranes for biological and medical applications. Chem. Soc. Rev. 2016, 45, 377-411. [CrossRef] [PubMed]

384. Rideau, E.; Dimova, R.; Schwille, P.; Wurm, F.R.; Landfester, K. Liposomes and polymersomes: A comparative review towards cell mimicking. Chem. Soc. Rev. 2018, 47, 8572-8610. [CrossRef]

385. Hu, X.; Zhang, Y.; Xie, Z.; Jing, X.; Bellotti, A.; Gu, Z. Stimuli-Responsive Polymersomes for Biomedical Applications. Biomacromolecules 2017, 18, 649-673. [CrossRef]

386. Einfalt, T.; Witzigmann, D.; Edlinger, C.; Sieber, S.; Goers, R.; Najer, A.; Spulber, M.; Onaca-Fischer, O.; Huwyler, J.; Palivan, C.G. Biomimetic artificial organelles with in vitro and in vivo activity triggered by reduction in microenvironment. Nat. Commun. 2018, 9, 1127. [CrossRef] [PubMed] 
387. van Oppen, L.M.P.E.; Abdelmohsen, L.K.E.A.; van Emst-de Vries, S.E.; Welzen, P.L.W.; Wilson, D.A.; Smeitink, J.A.M.; Koopman, W.J.H.; Brock, R.; Willems, P.H.G.M.; Williams, D.S.; et al. Biodegradable Synthetic Organelles Demonstrate ROS Shielding in Human-Complex-I-Deficient Fibroplasts. ACS Cent. Sci. 2018, 4, 917-928. [CrossRef]

388. Paprocki, D.; Koszelewski, D.; Walde, P.; Ostaszewski, R. Efficient Passerini reactions in an aqueous vesicle system. RSC Adv. 2015, 5, 102828-102835. [CrossRef]

389. Luginbühl, S.; Bertschi, L.; Willeke, M.; Schuler, L.D.; Walde, P. How Anionic Vesicles Steer the Oligomerization of Enzymatically Oxidized $p$-Aminodiphenylamine (PADPA) toward a Polyaniline Emeraldine Salt (PANI-ES)-Type Product. Langmuir 2016, 32, 9765-9779. [CrossRef]

390. Okada, S.; Peng, S.; Spevak, W.; Charych, D. Color and Chromism of Polydiacetylene Vesicles. Acc. Chem. Res. 1998, 31, 229-239. [CrossRef]

391. Lebègue, E.; Farre, C.; Jose, C.; Saulnier, J.; Lagarde, F.; Chevalier, Y.; Chaix, C.; Jaffrezic-Renault, N. Responsive Polydiacetylene Vesicles for Biosensing Microorganisms. Sensors 2018, 18, 599. [CrossRef] [PubMed]

392. Qian, X.; Städler, B. Recent Developments in Polydiacetylene-Based Sensors. Chem. Mater. 2019, 31, 1196-1222. [CrossRef]

393. Wang, D.-E.; Gao, X.; Li, G.; Xue, T.; Yang, H.; Xu, H. Facile colorimetric assay of alkaline phosphatase activity using polydiacetylene liposomes with calcium ions and pyrophosphate. Sens. Actuators B 2019, 289, 85-92. [CrossRef]

394. Handjani-Vila, R.M.; Ribier, A.; Rondot, B.; Vanlerberghie, G. Dispersions of lamellar phases of non-ionic lipids in cosmetic products. Int. J. Cosmet. Sci. 1979, 1, 303-314. [CrossRef] [PubMed]

395. Matoori, S.; Leroux, J.-C. Twenty-five years of polymersomes: Lost in translation? Mater. Horiz. 2020, 7, 1297-1309. [CrossRef]

396. Dou, Y.; Hynynen, K.; Allen, C. To heat or not to heat: Challenges with clinical translation of thermosensitive liposomes. J. Control. Release 2017, 249, 63-73. [CrossRef] [PubMed]

397. Kitayama, H.; Takechi, Y.; Tamai, N.; Matsuki, H.; Yomota, C.; Saito, H. Thermotropic Phase Behavior of Hydrogenated Soybean Phosphatidylcholine-Cholesterol Binary Liposome Membrane. Chem. Pharm. Bull. 2014, 62, 58-63. [CrossRef] [PubMed]

398. Jensen, G.M. The care and feeding of a commercial liposomal product: Liposomal amphotericin B (AmBisome ${ }^{\circledR}$ ). J. Liposome Res. 2017, 27, 173-179. [CrossRef]

399. Zurbriggen, R. Immunostimulating reconstituted influenza virosomes. Vaccine 2003, 21, 921-924. [CrossRef]

400. Moser, C.; Amacker, M.; Zurbriggen, R. Influenza virosomes as a vaccine adjuvant and carrier system. Expert Rev. Vaccines 2011, 10, 437-446. [CrossRef] [PubMed]

401. Glück, R. Immunopotentiating Reconstituted Influenza Virosomes (IRIVs). In Vaccine Adjuvants. Preparation Methods and Research Protocols; O’Hagan, D.T., Ed.; Methods in Molecular Medicine; Humana Press: Totowa, NJ, USA, 2000; Volume 42, Chapter 9; pp. 151-178.

402. Mischler, R.; Metcalfe, I.C. Inflexal ${ }^{\circledR}$ V a trivalent virosome subunit influenza vaccine: Production. Vaccine 2002, 20 , B17-B23. [CrossRef]

403. Gouma, S.; Anderson, E.M.; Hensley, S.E. Challenges of Making Effective Influenza Vaccines. Annu. Rev. Virol. 2020, 7, 495-512. [CrossRef] [PubMed]

404. Bayat, F.; Hosseinpour-Moghadam, R.; Mehryab, F.; Fatahi, Y.; Shakeri, N.; Dinarvand, R.; Ten Hagen, T.L.M.; Haeri, A. potential application of liposomal nanodevices for non-cancer diseases: An update on design, characterization and biopharmaceutical evaluation. Adv. Colloid Interface Sci. 2020, 277, 102121. [CrossRef] [PubMed]

405. Williams, A.S.; Camilleri, J.P.; Goodfellow, R.M.; Williams, D.D. A single intra-articular injection of liposomally conjugated methotrexate suppresses joint inflammation in rat antigen-induced arthritis. J. Rheumatol. 1996, 35, 719-724. [CrossRef] [PubMed]

406. Barrera, P.; Blom, A.; van Lent, P.L.E.M.; van Bloois, L.; Beijnen, J.H.; van Rooijen, N.; de Waal Malefijt, M.C.; van de Putte, L.B.A.; Storm, G.; van den Berg, W.B. Synovial Macrophage Depletion with Clodronate-containing Liposomes in Rheumatoid Arthritis. Arthritis Rheum. 2000, 43, 1951-1959. [CrossRef]

407. van den Hoven, J.M.; Van Tomme, S.R.; Metselaar, J.M.; Nuijen, B.; Beijnen, J.H.; Storm, G. Liposomal Drug Formulations in the Treatment of Rhematoid Arthritis. Mol. Pharm. 2011, 8, 1002-1015. [CrossRef]

408. Kapoor, B.; Singh, S.K.; Gulati, M.; Gupta, R.; Vaidya, Y. Application of Liposomes in Treatment of Rheumatoid Arthritis: Quo Vadis. Sci. World J. 2014, 978351. [CrossRef] [PubMed]

409. Forster, V.; Signorell, R.D.; Roveri, M.; Leroux, J.-C. Liposome-supported peritoneal dialysis for detoxification of drugs and endogenous metabolites. Sci. Transl. Med. 2014, 6, 258ra141. [CrossRef] [PubMed]

410. Matoori, S.; Forster, V.; Agostini, V.; Bettschart-Wolfensberger, R.; Bektas, R.N.; Thöny, B.; Häberle, J.; Leroux, J.-C. Preclinical evaluation of liposome-supported peritoneal dialysis for the treatment of hyperammonemic crisis. J. Control. Release 2020, 328, 503-513. [CrossRef] [PubMed]

411. Landon, C.D.; Park, J.-Y.; Needham, D.; Dewhirst, M.W. Nanoscale Drug Delivery and Hyperthermia: The Materials Design and Preclinical and Clinical Testing of Low Temperature-Sensitive Liposomes Used in Combination with Mild Hyperthermia in the Treatment of Local Cancer. Open Nanomed. J. 2011, 3, 38-64. [CrossRef]

412. May, J.P.; Li, S.-D. Hyperthermia-induced drug targeting. Expert Opin. Drug Deliv. 2013, 10, 511-527. [CrossRef] [PubMed]

413. Hynynen, K. Hyperthermia-induced drug delivery in humans. Nat. Biomed. Eng. 2018, 2, 637-639. [CrossRef]

414. Needham, D.; Anyarambhatla, G.; Kong, G.; Dewhirst, M.W. A New Temperature-sensitive Liposome for Use with Mild Hyperthermia: Characterization and Testing in a Human Tumor Xenograft Model. Cancer Res. 2000, 60, 1197-1201. [PubMed] 
415. Yatvin, M.B.; Weinstein, J.N.; Dennis, W.H.; Blumenthal, R. Design of Liposomes for Enhanced Local Release of Drugs by Hyperthermia. Science 1978, 202, 1290-1293. [CrossRef]

416. Weinstein, J.N.; Magin, R.L.; Yatvin, M.B.; Zaharko, D.S. Liposomes and Local Hyperthermia: Selective Delivery of Methotrexate to Heated Tumors. Science 1979, 204, 188-191. [CrossRef]

417. Needham, D. Bringing Research to Clinical Application. Lessons from ThermoDox ${ }^{\circledR}$ : A Thermal-Sensitive Liposome for Treatment of Cancer. In Drug Delivery. Fundamentals \& Applications, 2nd ed.; Hillery, A., Park, K., Eds.; CRC Press: Boca Raton, FL, USA, 2016; Chapter 23; pp. 523-585.

418. Chander, N.; Morstein, J.; Bolten, J.S.; Shemet, A.; Cullis, P.R.; Trauner, D.; Witzigmann, D. Optimized Photoactivatable Lipid Nanoparticles Enable Red Light Triggered Drug Release. Small 2021, 17, 2008198. [CrossRef] [PubMed]

419. Torchilin, V. Multifunctional and stimuli-sensitive pharmaceutical nanocarriers. Eur. J. Pharm. Biopharm. 2009, 71, 431-444. [CrossRef]

420. Lou, J.; Best, M.D. Strategies for altering lipid self-assembly to trigger liposome cargo release. Chem. Phys. Lipids 2020, 232, 104966. [CrossRef] [PubMed]

421. Yuba, E. Development of functional liposomes by modification of stimuli-responsive materials and their biomedical applications. J. Mater. Chem. B 2020, 8, 1093-1107. [CrossRef] [PubMed]

422. Davidsen, J.; Jørgensen, K.; Andresen, T.L.; Mouritsen, O.G. Secreted phospholipase $\mathrm{A}_{2}$ as a new enzymatic trigger mechanism for localised drug release and absorption in diseased tissue. Biochim. Biophys. Acta 2003, 1609, 95-101. [CrossRef]

423. Arouri, A.; Mouritsen, O.G. Phospholipase $\mathrm{A}_{2}$-susceptible liposomes of anticancer double lipid-prodrugs. Eur. J. Pharm. Sci. 2012, 45, 408-420. [CrossRef]

424. Hansen, A.H.; Mouritsen, O.G.; Arouri, A. Enzymatic action of phospholipase $\mathrm{A}_{2}$ on liposomal drug delivery. Int. J. Pharm. 2015, 491, 49-57. [CrossRef]

425. Cullis, P.R.; Hope, M.J. Lipid Nanoparticle Systems for Enabling Gene Therapies. Mol. Ther. 2017, 25, 1467-1475. [CrossRef] [PubMed]

426. Kulkarni, J.A.; Witzigmann, D.; Leung, J.; van der Meel, R.; Zaifman, J.; Darjuan, M.M.; Grisch-Chan, H.M.; Thöny, B.; Tam, Y.Y.C.; Cullis, P.R. Fusion-dependent formation of lipid nanoparticles containing macromolecular payloads. Nanoscale 2019, 11, 9023-9031. [CrossRef] [PubMed]

427. Carrasco, M.J.; Alishetty, S.; Alameh, M.-G.; Said, H.; Wright, L.; Paige, M.; Soliman, O.; Weissman, D.; Cleveland IV, T.E.; Grishaev, A.; et al. Ionization and structural properties of mRNA lipid nanoparticles influence expression in intramuscular and intravascular administration. Commun. Biol. 2021, 4, 956. [CrossRef]

428. Evers, M.J.W.; Kulkarni, J.A.; van der Meel, R.; Cullis, P.R.; Vader, P.; Schifflers, R.M. State-of-the Art Design and Rapid-Mixing Production Techniques of Lipid Nanoparticles for Nucleic Acid Delivery. Small Methods 2018, 2, 1700375. [CrossRef]

429. Kulkarni, J.A.; Witzigmann, D.; Chen, S.; Cullis, P.R.; van der Meel, R. Lipid Nanoparticle Technology for Clinical Translation of siRNA Therapeutics. Acc. Chem. Res. 2019, 52, 2435-2444. [CrossRef]

430. Kulkarni, J.A.; Darjuan, M.M.; Mercer, J.E.; Chen, S.; van der Meel, R.; Thewalt, J.L.; Tam, Y.Y.C.; Cullis, P.R. On the Formation and Morphology of Lipid Nanoparticles Containing Ionizable Cationic Lipids and siRNA. ACS Nano 2018, 12, 4787-4795. [CrossRef] [PubMed]

431. Kulkarni, J.A.; Cullis, P.R.; van der Meel, R. Lipid Nanoparticles Enabling Gene Therapies: From Concepts to Clinical Utility. Nucleic Acids Ther. 2018, 28, 146-157. [CrossRef]

432. Kulkarni, J.A.; Witzigmann, D.; Leung, J.; Tam, Y.Y.C.; Cullis, P.R. On the role of helper lipids in lipid nanoparticle formulations of siRNA. Nanoscale 2019, 11, 21733-21739. [CrossRef] [PubMed]

433. Terada, T.; Kulkarni, J.A.; Huynh, A.; Chen, S.; van der Meel, R.; Tam, Y.Y.C.; Cullis, P.R. Characterization of Lipid Nanoparticles Containing Ionizable Cationic Lipids Using Design-of-Experiments Approach. Langmuir 2021, 37, 1120-1128. [CrossRef] [PubMed]

434. Lv, H.; Zhang, S.; Wang, B.; Cui, S.; Yan, J. Toxicity of cationic lipids and cationic polymers in gene delivery. J. Control. Release 2006, 114, 100-109. [CrossRef] [PubMed]

435. Cui, S.; Wang, Y.; Gong, Y.; Lin, X.; Zhao, Y.; Zhi, D.; Zhou, Q.; Zhang, S. Correlation of the cytotoxic effects of cationic lipids with their headgroups. Toxicol. Res. 2018, 7, 473-479. [CrossRef] [PubMed]

436. Witzigmann, D.; Kulkarni, J.A.; Leung, J.; Chen, S.; Cullis, P.R.; van der Meel, R. Lipid nanoparticle technology for therapeutic gene regulation in the liver. Adv. Drug Deliv. Rev. 2020, 159, 344-363. [CrossRef]

437. Kulkarni, J.A.; Thomson, S.B.; Zaifman, J.; Leung, J.; Wagner, P.K.; Hill, A.; Tam, Y.Y.C.; Cullis, P.R.; Petkau, T.L.; Leavitt, B.R. Spontaneous, solvent-free entrapment of siRNA within lipid nanoparticles. Nanoscale 2020, 12, 23959-23966. [CrossRef] [PubMed]

438. Reichmuth, A.M.; Oberli, M.A.; Jaklenec, A.; Langer, R.; Blankschtein, D. mRNA vaccine delivers using lipid nanoparticles. Ther. Deliv. 2016, 7, 319-334. [CrossRef] [PubMed]

439. Pardi, N.; Hogan, M.J.; Porter, F.W.; Weissman, D. mRNA vaccines-A new era in vaccinology. Nat. Rev. Drug Discov. 2018, 17, 261-279. [CrossRef] [PubMed]

440. Kim, J.; Eygeris, Y.; Gupta, M.; Sahay, G. Self-assembled mRNA vaccines. Adv. Drug Deliv. Rev. 2021, 170, 83-112. [CrossRef] [PubMed] 
441. Viger-Gravel, J.; Schantz, A.; Pinon, A.C.; Rossini, A.J.; Schantz, S.; Emsley, L. Structure of Lipid Nanoparticles Containing siRNA or mRNA by Dynamic Nuclear Polarization-Enhanced NMR Spectroscopy. J. Phys. Chem. B 2018, 122, 2073-2081. [CrossRef] [PubMed]

442. Rozmanov, D.; Baoukina, S.; Tieleman, D.P. Density Based Visualization for Molecular Simulation. Faraday Discuss. 2014, 169, 225-243. [CrossRef] [PubMed]

443. Leung, A.K.K.; Hafez, I.M.; Baoukina, S.; Belliveau, N.M.; Zhigaltsev, I.V.; Afshinmanesh, E.; Tieleman, D.P.; Hansen, C.L.; Hope, M.J.; Cullis, P.R. Lipid nanoparticles containing siRNA synthesized by microfluidic mixing exhibit an electron-dense nanostructured core. J. Phys. Chem. C 2012, 116, 18440-18450. [CrossRef]

444. Oberli, M.A.; Reichmuth, A.M.; Dorkin, J.R.; Mitchell, M.J.; Fenton, O.S.; Jaklenec, A.; Anderson, D.G.; Langer, R.; Blankschtein, D. Lipid Nanoparticle Assisted mRNA Delivery for Potent Cancer Immunotherapy. Nano Lett. 2017, 17, 1326-1335. [CrossRef] [PubMed]

445. Brader, M.L.; Williams, S.J.; Banks, J.M.; Hui, W.H.; Zhou, Z.H.; Jin, L. Encapsulation state of messenger RNA inside lipid nanoparticles. Biophys. J. 2021, 120, 1-5. [CrossRef] [PubMed]

446. Noor, R. Development Status of the Potential Vaccines for the Mitigation of the COVID-19 Pandemic and a Focus on the Effectiveness of the Pfizer-BioNTech and Moderna mRNA Vaccines. Curr. Clin. Microbiol. Rep. 2021, 8, 178-185. [CrossRef] [PubMed]

447. Gao, Y.; Yang, K.; Shelling, A.N.; Wu, Z. Nanotechnology-Enabled COVID-19 mRNA Vaccines. Encyclopedia 2021, 1, 773-780. [CrossRef]

448. Cevc, G.; Schätzlein, A.G.; Richardsen, H.; Vierl, U. Overcoming Semipermeable Barriers, Such as the Skin, with Ultradeformable Mixed Lipid Vesicles, Transfersomes, Liposomes, or Mixed Lipid Micelles. Langmuir 2003, 19, 10753-10763. [CrossRef]

449. Benson, H.A.E. Elastic Liposomes for Topical and Transdermal Drug Delivery. In Liposomes. Methods and Protocols; Weissig, V., Ed.; Volume 1: Pharmaceutical Nanocarriers; Humana Press: New York, USA, 2010; Chapter 4; pp. 77-86.

450. Ashtikar, M.; Nagarsekar, K.; Fahr, A. Transdermal delivery from liposomal formulations-Evolution of the technology over the last three decades. J. Control. Release 2016, 242, 126-140. [CrossRef]

451. Mirtaleb, M.S.; Shahraky, M.K.; Ekrami, E.; Mirtaleb, A. Advances in biological nano-phospholipid vesicles for transdermal delivery: A review on applications. J. Drug Deliv. Sci. Technol. 2021, 61, 102331. [CrossRef]

452. Fernández-García, R.; Lalatsa, A.; Statts, L.; Bolás-Fernández, F.; Ballesteros, M.P.; Serrano, D.R. Transfersomes as nanocarriers for drugs across the skin: Quality by design from lab to industrial scale. Int. J. Pharm. 2020, 573, 118817. [CrossRef] [PubMed]

453. Uhl, P.; Sauter, M.; Hertlein, T.; Witzigmann, D.; Laffleur, F.; Hofhaus, G.; Fidelj, V.; Tursch, A.; Özbek, S.; Hopke, E.; et al. Overcoming the Mucosal Barrier: Tetraether Lipid-Stabilized Liposomal Nanocarriers Decorated with Cell-Penetrating Peptides Enable Oral Delivery of Vancomycin. Adv. Therap. 2021, 4, 2000247. [CrossRef]

454. Chong, P.L.-G.; Ayesa, U.; Daswani, V.P.; Hur, E.C. On the Physical Properties of Tetraether Lipid Membranes: Effects of Cyclopentane Rings. Archea 2012, 2012, 138439. [CrossRef] [PubMed]

455. Uhl, P.; Helm, F.; Hofhaus, G.; Brings, S.; Kaufman, C.; Leotta, K.; Urban, S.; Haberkorn, U.; Mier, W.; Fricker, G. A liposomal formulation for the oral application of the investigational hepatitis B drug Myrcludex B. Eur. J. Pharm. Biopharm. 2016, 103, 159-166. [CrossRef]

456. Massing, U.; Cicko, S.; Ziroli, V. Dual asymmetric centrifugation (DAC)—A new technique for liposome preparation. J. Control. Release 2008, 125, 16-24. [CrossRef] [PubMed]

457. Uhl, P.; Pantze, S.; Storck, P.; Parmentier, J.; Witzigmann, D.; Hofhaus, G.; Huwyler, J.; Mier, W.; Fricker, G. Oral delivery of vancomycin by tetraether lipid liposomes. Eur. J. Pharm. Sci. 2017, 108, 111-118. [CrossRef] [PubMed]

458. Xu, Y.; Bohns Michalowski, C.; Beloqui, A. Advances in lipid carriers for drug delivery to the gastrointestinal tract. Curr. Opin. Colloid Interface Sci. 2021, 52, 101414. [CrossRef]

459. Gomez, A.G.; Hosseinidoust, Z. Liposomes for Antibiotic Encapsulation and Delivery. ACS Infect. Dis. 2020, 6, 896-908. [CrossRef] [PubMed]

460. Vamvakaki, V.; Chaniotakis, N.A. Pesticide detection with a liposome-based nano-biosensor. Biosens. Bioelectron. 2007, 22, 2848-2853. [CrossRef]

461. Liu, Q.; Boyd, B.J. Liposomes in biosensors. Analyst 2013, 138, 391-409. [CrossRef]

462. Mazur, F.; Bally, M.; Städler, B.; Chandrawati, R. Liposomes and lipid bilayers in biosensors. Adv. Colloid Interface Sci. 2017, 249, 88-99. [CrossRef]

463. Hofmann, C.; Duerkop, A.; Baeumner, A.J. Nanocontainers for Analytical Applications. Angew. Chem. Int. Ed. 2019, 58, 12840-12860. [CrossRef]

464. Sforzi, J.; Palagi, L.; Aime, S. Liposome-Based Bioassays. Biology 2020, 9, 202. [CrossRef] [PubMed]

465. Edwards, K.A.; Bolduc, O.R.; Baeumner, A.J. Miniaturized bioanalytical systems: Enhanced performance through liposomes. Curr. Opin. Chem. Biol. 2012, 16, 444-452. [CrossRef] [PubMed]

466. Sforzi, J.; Ferrauto, G.; Aime, S.; Geninatti Crich, S. A Simple and Fast Assay Based on Carboxyfluorescein-Loaded Liposome for Quantitative DNA Detection. ACS Omega 2020, 5, 1764-1772. [CrossRef] [PubMed]

467. Mazur, F.; Tran, H.; Kuchel, R.P.; Chandrawati, R. Rapid Detection of Listeriolysin O Toxin Based on a Nanoscale Liposome-Gold Nanoparticle Platform. ACS Appl. Nano Mater. 2020, 3, 7270-7280. [CrossRef] 
468. Beigi, F.; Yang, Q.; Lundahl, P. Immobilized-liposome chromatographic analysis of drug partitioning into lipid bilayers. J. Chromatogr. A 1995, 704, 315-321. [CrossRef]

469. Yang, Q.; Liu, X.-Y.; Yoshimoto, M.; Kuboi, R.; Miyake, J. Covalent Immobilization of Unilamellar Liposomes in Gel Beads for Chromatography. Anal. Biochem. 1999, 268, 354-362. [CrossRef]

470. Liu, X.-Y.; Yang, Q.; Nakamura, C.; Miyake, J. Avidin-biotin-immobilized liposome column for chromatographic fluorescence on-line analysis of solute-membrane interactions. J. Chromatogr. A 2001, 750, 51-60. [CrossRef]

471. Dusseillier, M.R.; Niederberger, B.; Städler, B.; Falconnet, D.; Textor, M.; Vörös, J. A novel crossed microfluidic device for the precise positioning of proteins and vesicles. Lab Chip 2005, 5, 1387-1392. [CrossRef] [PubMed]

472. Bally, M.; Bailey, K.; Sugihara, K.; Grieshaber, D.; Vörös, J.; Städler, B. Liposome and Lipid Bilayer Arrays Towards Biosensing Applications. Small 2010, 6, 2481-2497. [CrossRef] [PubMed]

473. Hosta-Rigau, L.; Zhang, Y.; Teo, B.M.; Postma, A.; Städler, B. Cholesterol—a biological compound as a building block in bionanotechnology. Nanoscale 2013, 5, 89-109. [CrossRef]

474. Chen, R.F.; Knutson, J.R. Mechanism of Fluorescence Concentration Quenching of Carboxyfluorescein in Liposomes: Energy Transfer to Nonfluorescent Dimers. Anal. Biochem. 1988, 172, 61-77. [CrossRef]

475. Nasr, G.; Greige-Gerges, H.; Elasissari, A.; Khreich, N. Liposomal membrane permeability assessment by fluorescent techniques: Main permeabilizing agents, applications and challenges. Int. J. Pharm. 2020, 580, 119198. [CrossRef]

476. Schnitzer, E.; Lichtenberg, D.; Kozlov, M.M. Temperature-dependence of the solubilization of dipalmitoylphosphatidylcholine (DPPC) by the non-ionic surfactant Triton X-100, kinetic and structural aspects. Chem. Phys. Lipids 2003, 126, 55-76. [CrossRef]

477. Schnitzer, E.; Kozlov, M.M.; Lichtenberg, D. The effect of cholesterol on the solubilization of phosphatidylcholine bilayers by the non-ionic surfactant Triton X-100. Chem. Phys. Lipids 2005, 135, 69-82. [CrossRef] [PubMed]

478. Dalgarno, P.A.; Juan-Cólas, J.; Hedley, G.J.; Piñeiro, L.; Novo, M.; Perez-Gonzalez, C.; Samuel, I.D.W.; Leake, M.C.; Johnson, S.; Al-Soufi, W.; et al. Unveiling the multi-step solubilization mechansim of sub-micron size vesicles by detergents. Sci. Rep. 2019, 9, 12897. [CrossRef] [PubMed]

479. Gómez-Hens, A.; Fernández-Romero, J.M. The role of liposomes in analytical processes. Trends Anal. Chem. 2005, 24, 9-19. [CrossRef]

480. Lin, C.; Guo, Y.; Zhao, M.; Sun, M.; Luo, F.; Guo, L.; Qiu, B.; Lin, Z.; Chen, G. Highly sensitive colorimetric immunosensor for influenza virus H5N1 based on enzyme-encapsulated liposome. Anal. Chim. Acta 2017, 963, 112-118. [CrossRef] [PubMed]

481. Josephy, P.D.; Eling, T.; Mason, R.P. Horseradish Peroxidase-catalyzed Oxidations of 3,5,3' ,5'-Tetramethylbenzidine. J. Biol. Chem. 1982, 257, 3669-3675. [CrossRef]

482. Yoshimoto, M.; Higa, M. A kinetic analysis of catalytic production of oxygen in catalase-containing liposome dispersions for controlled transfer of oxygen in a bioreactor. J. Chem. Technol. Biotechnol. 2014, 89, 1388-1395. [CrossRef]

483. Beck, S.M.; Brus, L.E. Transient Spontaneous Raman Study of Photoionization Kinetics at the Hydrocarbon/Water Interface in Micellar Solutions. J. Am. Chem. Soc. 1983, 105, 1106-1111. [CrossRef]

484. Umena, Y.; Yorita, K.; Matsuoka, T.; Kita, A.; Fukui, K.; Morimoto, Y. The crystal structure of L-lactate oxidase from Aerococcus viridans at $2.1 \AA$ resolution reveals the mechanism of strict substrate recognition. Biochem. Biophys. Res. Commun. 2006, 350, 249-256. [CrossRef]

485. Maeda-Yorita, K.; Aki, K.; Sagai, H.; Misaki, H.; Massey, V. L-Lactate oxidase and L-lactate monooxygenase: Mechanistic variations on a common structural theme. Biochimie 1995, 77, 631-642. [CrossRef]

486. Matoori, S.; Mooney, D.J. Near-Infrared Fluorescence Hydrogen Peroxide Assay for Versatile Metabolite Biosensing in Whole Blood. Small 2020, 16, 2000369. [CrossRef] [PubMed]

487. Gabba, M.; Frallicciardi, J.; van't Klooster, J.; Henderson, R.; Syga, Ł.; Mans, R.; van Maris, A.J.A.; Poolman, B. Weak Acid Permeation in Synthetic Lipid Vesicles and Across the Yeast Plasma Membrane. Biophys. J. 2020, 118, 422-434. [CrossRef] [PubMed]

488. Taylor, T.M.; Davidson, P.M.; Bruce, B.D.; Weiss, J. Liposomal Nanocapsules in Food Science and Agriculture. Crit. Rev. Food Sci. Nutr. 2005, 45, 587-605. [CrossRef] [PubMed]

489. Keller, B.C. Liposomes in nutrition. Trends Food Sci. Technol. 2001, 12, 25-31. [CrossRef]

490. Singh, H.; Thompson, A.; Liu, W.; Corredig, M. Liposoms as food ingredients and nutraceutical delivery systems. In Encapsulation Technologies and Delivery Systems for Food Ingredients and Nutraceuticals; Garti, N., McClements, D.J., Eds.; Woodhead Publishing: Oxford, UK, 2012; Chapter 11; pp. 288-318.

491. Khorasani, S.; Danaei, M.; Mozafari, M.R. Nanoliposome technology for the food and nutraceutical industries. Trends Food Sci. Technol. 2018, 79, 106-115. [CrossRef]

492. Zhao, L.; Temelli, F.; Chen, L. Encapsulation of anthocyanin in liposomes using supercritical carbon dioxide: Effects of anthocyanin and sterol concentrations. J. Funct. Foods 2017, 34, 159-167. [CrossRef]

493. Marín, D.; Alemán, A.; Montero, P.; Gómez-Guillén, M.C. Encapsulation of food waste compounds in soy phosphatidylcholine liposomes: Effect of freeze-drying, storage stability and functional aptitude. J. Food Eng. 2018, 223, 132-143. [CrossRef]

494. Kheadr, E.E.; Vuillemard, J.-C.; El Deeb, S.A. Accelerated Cheddar cheese ripening with encapsulated proteinases. Int. J. Food Sci. Technol. 2000, 35, 483-495. [CrossRef]

495. Kheadr, E.E.; Vuillemard, J.-C.; El-Deeb, S.A. Impact of liposome-encapsulated enzyme cocktails on cheddar cheese ripening. Food Res. Int. 2003, 36, 241-252. [CrossRef] 
496. da Silva Malheiros, P.; Daroit, D.J.; Brandelli, A. Food applications of liposome-encapsulated antimicrobial peptides. Trends Food Sci. Technol. 2010, 21, 284-292. [CrossRef]

497. Vergara, D.; López, O.; Bustamante, M.; Shene, C. An in vitro digestion study of encapsulated lactoferrin in rapeseed phospholipidbased liposomes. Food Chem. 2020, 321, 126717. [CrossRef] [PubMed]

498. García-Toledo, J.A.; Torrestiana-Sánchez, B.; Martínez-Sánchez, C.E.; Tejero-Andrade, J.M.; García-Bórquez, A.; Mendoza-García, P.G. Nanoencapsulation of a Bacteriocin from Pediococcus acidilactici ITV26 by Microfluidization. Food Bioprocess Technol. 2019, 12, 88-97. [CrossRef]

499. Liu, X.; Wang, P.; Zou, Y.-X.; Luo, Z.-G.; Tamer, T.M. Co-encapsulation of Vitamin C and $\beta$-Carotene in liposomes: Storage stability, antioxidant activity, and in vitro gastrointestinal digestion. Food Res. Int. 2020, 136, 109587. [CrossRef] [PubMed]

500. Paolino, D.; Mancuso, A.; Cristiano, M.C.; Froiio, F.; Lammari, N.; Celia, C.; Fresta, M. Nanonutraceuticals: The New Frontier of Supplementary Food. Nanomaterials 2021, 11, 792. [CrossRef] [PubMed]

501. Emani, S.; Azadmard-Damirichi, S.; Peighambardoust, S.H.; Valizadeh, H.; Hesari, J. Liposomes as carrier vehicles for functional compounds in food sector. J. Exp. Nanosci. 2016, 11, 737-759. [CrossRef]

502. Shukla, S.; Haldorai, Y.; Hwang, S.K.; Bajpai, V.K.; Huh, Y.S.; Han, Y.-K. Current Demands for Food-Approved Liposome Nanoparticles in Food and Safety Sector. Front. Microbiol. 2017, 8, 02398. [CrossRef] [PubMed]

503. Hasibi, F.; Nasirouir, A.; Varshosaz, J.; García-Manrique, P.; Blanco-López, M.C.; Gutiérrez, G.; Matos, M. Formulation and Characterization of Taxifolin-Loaded Lipid Nanovesicles (Liposomes, Niosomes, and Transfersomes) for Beverage Fortification. Eur. J. Lipid Sci. Technol. 2020, 122, 1900105. [CrossRef]

504. Subramani, T.; Ganapathyswamy, H. An overview of liposomal nano-encapsulation techniques and its applications in food and nutraceutical. J. Food Sci. Technol. 2020, 57, 3545-3555. [CrossRef] [PubMed]

505. Zarrabi, A.; Abadi, M.A.A.; Khorasani, S.; Mohammadabadi, M.-R.; Jamshidi, A.; Torkaman, S.; Taghavi, E.; Mozafari, M.R.; Rasti, B. Nanoliposomes and Tocosomes as Multifunctional Nanocarriers for the Encapsulation of Nutraceutical and Dietary Molecules. Molecules 2020, 25, 638. [CrossRef] [PubMed]

506. Blesso, C.N. Egg Phospholipids and Cardiovascular Health. Nutrients 2015, 7, 2731-2747. [CrossRef] [PubMed]

507. van der Veen, J.N.; Kennelly, J.P.; Wan, S.; Vance, J.E.; Vance, D.E.; Jacobs, R.L. The critical role of phosphatidylcholine and phosphatidylethanolamine metabolism in health and disease. Biochim. Biophys. Acta 2017, 1859, 1558-1572. [CrossRef] [PubMed]

508. Robert, C.; Couëdelo, L.; Vayasse, C.; Michalski, M.-C. Vegetable lecithins: A review of their compositional diversity, impact on lipid metabolism and potential in cardiometabolic disease prevention. Biochimie 2020, 169, 121-132. [CrossRef]

509. Kim, I.-S.; Kim, C.-H.; Yang, W.-S. Physiologically Active Molecules and Functional Properties of Soybeans in Human Health-A Current Perspective. Int. J. Mol. Sci. 2021, 22, 4054. [CrossRef] [PubMed]

510. Łukawski, M.; Dałek, P.; Borowik, T.; Foryś, A.; Langner, M.; Witkiewicz, W.; Przybło, M. New oral liposomal vitamin C formulation: Properties and bioavailability. J. Liposome Res. 2020, 30, 227-234. [CrossRef] [PubMed]

511. Prantl, L.; Eigenberger, A.; Gehmert, S.; Haerteis, S.; Aung, T.; Rachel, R.; Jung, E.M.; Felthaus, O. Enhanced Resorption of Liposomal Packed Vitamin C Monitored by Ultrasound. J. Clin. Med. 2020, 9, 1616. [CrossRef] [PubMed]

512. van Hoogevest, P. Review-An update on the use of oral phospholipid excipients. Eur. J. Pharm. Sci. 2017, 108, 1-12. [CrossRef] [PubMed]

513. Kayushin, L.P.; Skulachev, V.P. Bacteriorhodopsin as an Electrogenic Proton Pump: Reconstitution of Bacteriorhodopsin Proteoliposomes Generating $\Delta \psi$ and $\triangle \mathrm{pH}$. FEBS Lett. 1974, 39, 39-42. [CrossRef]

514. Eytan, G.D. Use of Liposomes for Reconstitution of Biological Functions. Biochim. Biophys. Acta 1982, 694, 185-202. [CrossRef]

515. Seddon, A.M.; Curnow, P.; Booth, P.J. Membrane proteins, lipids and detergents: Not just a soap opera. Biochim. Biophys. Acta 2004, 1666, 105-117. [CrossRef] [PubMed]

516. Gulik-Krzywicki, T.; Seigneuret, M.; Rigaud, J.L. Monomer-Oligomer Equilibrium of Bacteriorhodopsin in Reconstituted Proteoliposomes. A Freeze Fracture Electron Microscopy Study. J. Biol. Chem. 1987, 262, 15580-15588. [CrossRef]

517. Rigaud, J.-L.; Lévy, D. Reconstitution of Membrane Proteins into Liposomes. Methods Enzymol. 2003, $372,65-86$.

518. Walde, P.; Ichikawa, S.; Yoshimoto, M. The Fabrication and Applications of Enzyme-Containing Vesicles. In Bottom-Up Nanofabrication; Ariga, K., Nalwa, H.S., Eds.; American Scientific Publishers: Steveson Ranch, CA, USA, 2009; Volume 2, pp. $199-221$.

519. Lévy, D.; Gulik, A.; Bluzat, A.; Rigaud, J.-L. Reconstitution of the sarcoplasmic reticulum Ca ${ }^{2+}$-ATPase: Mechanisms of membrane protein insertion into liposomes during reconstitution procedures involving the use of detergents. Biochim. Biophys. Acta 1992, 1107, 283-298. [CrossRef]

520. Rigaud, J.-L.; Pitard, B.; Levy, D. Reconstitution of membrane proteins into liposomes: Application to energy-transducing membrane proteins. Biochim. Biophys. Acta 1995, 1231, 223-246. [CrossRef]

521. Girard, P.; Pécréaux, J.; Lenoir, G.; Falson, P.; Rigaud, J.-L.; Bassereau, P. A New Method for the Reconstitution of Membrane Proteins into Giant Unilamellar Vesicles. Biophys. J. 2004, 87, 419-429. [CrossRef]

522. Dezi, M.; Di Cicco, A.; Bassereau, P.; Lévy, D. Detergent-mediated incorporation of transmembrane proteins in giant unilamellar vesicles with controlled physiological contents. Proc. Natl. Acad. Sci. USA 2013, 110, 7276-7281. [CrossRef] [PubMed]

523. Jørgensen, I.L.; Kemmer, G.C.; Pomorski, T.G. Membrane protein reconstitution into giant unilamellar vesicles: A review on current techniques. Eur. Biophys. J. 2017, 46, 103-119. [CrossRef]

524. Amati, A.M.; Graf, S.; Deutschmann, S.; Dolder, N.; von Ballmoos, C. Current problems and future avenues in proteoliposome research. Biochem. Soc. Trans. 2020, 48, 1473-1492. [CrossRef] [PubMed] 
525. Garten, M.; Lévy, D.; Bassereau, P. Protein reconstitution in giant vesicles. In The Giant Vesicle Book; Dimova, R., Marques, C.M., Eds.; CRC Press, Taylor \& Francis Group: Boca Raton, FL, USA, 2020; Chapter 3; pp. 37-51.

526. Markones, M.; Fippel, A.; Kaiser, M.; Drechsler, C.; Hunte, C.; Heerklotz, H. Stairway to Asymmetry: Five Steps to LipidAsymmetric Proteoliposomes. Biophys. J. 2020, 118, 294-302. [CrossRef] [PubMed]

527. Yao, X.; Fan, X.; Yan, N. Cryo-EM analysis of a membrane protein embedded in the liposome. Proc. Natl. Acad. Sci. USA 2020, 117, 18497-18503. [CrossRef]

528. Murakami, S.; Nakashima, R.; Yamashita, E.; Yamaguchi, A. Crystal structure of bacterial multidrug efflux transporter AcrB. Nature 2002, 419, 587-593. [CrossRef]

529. Baumgart, T.; Hammond, A.T.; Sengupta, P.; Hess, S.T.; Holowka, D.A.; Baird, B.A.; Webb, W.W. Large-scale fluid/fluid phase separation of proteins and lipids in giant plasma membrane vesicles. Proc. Natl. Acad. Sci. USA 2007, 104, 3165-3170. [CrossRef] [PubMed]

530. Levental, I.; Grzybek, M.; Simons, K. Raft domains of variable properties and compositions in plasma membrane vesicles. Proc. Natl. Acad. Sci. USA 2011, 108, 11411-11416. [CrossRef] [PubMed]

531. Steinkühler, J.; Sezgin, E.; Urbančič, I.; Eggeling, C.; Dimova, R. Mechanical properties of plasma membrane vesicles correlate with lipid order, viscosity and cell density. Commun. Biol. 2019, 2, 337. [CrossRef] [PubMed]

532. Skinkle, A.D.; Levental, K.R.; Levental, I. Cell-Derived Plasma Membrane Vesicles Are Permeable to Hydrophilic Macromolecules. Biophys. J. 2020, 118, 1292-1300. [CrossRef] [PubMed]

533. Lorent, J.H.; Levental, I. Preparation and properties of giant plasma membrane vesicles and giant unilamellar vesicles from natural membranes. In The Giant Vesicle Book; Dimova, R., Marques, C.M., Eds.; CRC Press, Taylor \& Francis Group: Boca Raton, FL, USA, 2020; Chapter 2; pp. 21-36.

534. Srivatsav, A.T.; Kapoor, S. The Emerging World of Membrane Vesicles: Functional Relevance, Theranostic Avenues and Tools for Investigating Membrane Function. Front. Mol. Biosci. 2021, 8, 640355. [CrossRef]

535. Vlassov, A.V.; Magdaleno, S.; Setterquist, R.; Conrad, R. Exosomes: Current knowledge of their composition, biological functions, and diagnostic and therapeutic potentials. Biochim. Biophys. Acta 2012, 1820, 940-948. [CrossRef]

536. Raposo, G.; Stoorvogel, W. Extracellular vesicles: Exosomes, microvesicles, and friends. J. Cell Biol. 2013, 200, 373-383. [CrossRef]

537. Wiklander, O.P.B.; Brennan, M.Á.; Lötvall, J.; Breakefield, X.O.; Andaloussi, S.E.L. Advances in therapeutic applications of extracellular vesicles. Sci. Transl. Med. 2019, 11, eaav8521. [CrossRef] [PubMed]

538. Elsharkasy, O.M.; Nordin, J.Z.; Hagey, D.W.; de Jong, O.G.; Schiffelery, R.M.; Andaloussi, S.E.L.; Vader, P. Extracellular vesicles as drug delivery systems: Why and how? Adv. Drug Deliv. Rev. 2020, 159, 332-343. [CrossRef]

539. Gandham, S.; Su, X.; Wood, J.; Nocera, A.L.; Alli, S.C.; Milane, L.; Zimmerman, A.; Amiji, M.; Ivanov, A.R. Technologies and Standardization in Research on Extracellular Vesicles. Trends Biotechnol. 2020, 38, 1066-1098. [CrossRef] [PubMed]

540. Prosser, R.S.; Evanics, F.; Kitevski, J.L.; Al-Abdul-Wahid, M.S. Current Applications of Bicelles in NMR Studies of MembraneAssociated Amphiphiles and Proteins. Biochemistry 2006, 45, 8453-8465. [CrossRef] [PubMed]

541. Dürr, U.H.N.; Gildeberg, M.; Ramamoorthy, A. The Magic of Bicelles Lights Up Membrane Protein Structure. Chem. Rev. 2012, 112, 6054-6074. [CrossRef] [PubMed]

542. Morrison, E.A.; Henzler-Wildman, K.A. Reconstitution of integral membrane proteins into isotropic bicelles with improved sample and expanded lipid composition profile. Biochim. Biophys. Acta 2012, 1818, 814-820. [CrossRef] [PubMed]

543. Oberholzer, T.; Nierhaus, K.H.; Luisi, P.L. Protein Expression in Liposomes. Biochem. Biophys. Res. Commun. 1999, $261,238-241$. [CrossRef] [PubMed]

544. Nomura, S.M.; Tsumoto, K.; Hamada, T.; Akiyoshi, K.; Nakatani, Y.; Yoshikawa, K. Gene Expression within Cell-Sized Lipid Vesicles. ChemBioChem 2003, 4, 1172-1175. [CrossRef] [PubMed]

545. Ishikawa, K.; Sato, K.; Shima, Y.; Urabe, I.; Yomo, T. Expression of a cascading genetic network within liposomes. FEBS Lett. 2004, 576, 387-390. [CrossRef] [PubMed]

546. Luisi, P.L. Toward the Engineering of Minimal Living Cells. Anat. Rec. 2002, 268, 208-214. [CrossRef]

547. Stano, P. Is Research on "Synthetic Cells" Moving to the Next Level? Life 2019, 9, 3. [CrossRef] [PubMed]

548. Xu, C.; Hu, S.; Chen, X. Artificial cells: From basic science to applications. Mater. Today 2016, 19, 516-532. [CrossRef]

549. Buddingh, B.C.; van Hest, J.C.M. Artificial Cells: Synthetic Compartments with Life-like Functionality and Adaptivity. Acc. Chem. Res. 2017, 50, 769-777. [CrossRef] [PubMed]

550. Trantidou, T.; Friddin, M.; Elani, Y.; Brooks, N.J.; Law, R.V.; Seddon, J.M.; Ces, O. Engineering Compartmentalized Biomimetic Micro- and Nanocontainers. ACS Nano 2017, 11, 6549-6565. [CrossRef] [PubMed]

551. Yewdall, N.A.; Mason, A.F.; van Hest, J.C.M. The hallmarks of living systems: Towards creating artificial cells. Interface Focus 2018, 8, 20180023. [CrossRef] [PubMed]

552. Kai, L.; Schwille, P. Cell-Free Protein Synthesis and Its Perspectives for Assembling Cells from the Bottom-Up. Adv. Biosys. 2019, 3, 1800322. [CrossRef] [PubMed]

553. Lentini, R.; Martín, N.Y.; Forlin, M.; Belmonte, L.; Fontana, J.; Cornella, M.; Martini, L.; Tamburini, S.; Bentley, W.E.; Jousson, O.; et al. Two-Way Chemical Communication between Artificial and Natural Cells. ACS Cent. Sci. 2017, 3, 117-123. [CrossRef]

554. Kuruma, Y.; Stano, P.; Ueda, T.; Luisi, P.L. A synthetic biology approach to the construction of membrane proteins in semi-synthetic minimal cells. Biochim. Biophys. Acta 2009, 1788, 567-574. [CrossRef] [PubMed] 
555. Soga, H.; Fujii, S.; Yomo, T.; Kato, Y.; Watanabe, H.; Matsuura, T. In Vitro Membrane Protein Synthesis Inside Cell-Sized Vesicles Reveals the Dependence of Membrane Protein Integration on Vesicle Volume. ACS Synth. Biol. 2014, 3, 372-379. [CrossRef]

556. Altamura, A.; Milano, F.; Tangorra, R.R.; Trotta, M.; Omar, O.H.; Stano, P.; Mavelli, F. Highly oriented photosynthetic reaction centers generate a proton gradient in synthetic protocells. Proc. Natl. Acad. Sci. USA 2017, 114, 3837-3842. [CrossRef] [PubMed]

557. Altamura, E.; Albanese, P.; Marotta, R.; Milano, F.; Fiore, M.; Trotta, M.; Stano, P.; Mavelli, F. Chromatophores efficiently promote light-driven ATP synthesis and DNA transcription inside hybrid multicompartment artificial cells. Proc. Natl. Acad. Sci. USA 2021, 118, e2012170118. [CrossRef] [PubMed]

558. Gánti, T. Biogenesis Itself. J. Theor. Biol. 1997, 187, 583-593. [CrossRef]

559. Gánti, T. The Principles of Life: With a Commentary by James Griesemer and Eörs Szathmáry; Oxford University Press: Oxford, UK, 2003.

560. Griesemer, J. The enduring value of Gánti's chemoton model and life criteria: Heuristic pursuit of exact theoretical biology. J. Theor. Biol. 2015, 381, 23-28. [CrossRef] [PubMed]

561. Gibson, D.G.; Glass, J.I.; Lartigue, C.; Noskov, V.N.; Chuang, R.-Y.; Algire, M.A.; Benders, G.A.; Montague, M.G.; Ma, L.; Moodie, M.M.; et al. Creation of a Bacterial Cell Controlled by a Chemically Synthesized Genome. Science 2010, 329, 52-56. [CrossRef] [PubMed]

562. Shimizu, Y.; Inoue, A.; Tomari, Y.; Suzuki, T.; Yokogawa, T.; Nishikawa, K.; Ueda, T. cell-free translation reconstitution with purified components. Nat. Biotechnol. 2001, 19,751-755. [CrossRef]

563. Kuruma, Y.; Ueda, T. The PURE system for the cell-free synthesis of membrane proteins. Nat. Protoc. 2015, 10, 1328-1344. [CrossRef] [PubMed]

564. Luisi, P.L. About Various Definitions of Life. Origins Life Evol. Biospheres 1998, 28, 613-622. [CrossRef]

565. Cleland, C.E.; Chyba, C.F. Defining 'Life'. Origins Life Evol. Biospheres 2002, 32, 387-393. [CrossRef] [PubMed]

566. Ruiz-Mirazo, K.; Peretó, J.; Moreno, A. A Universal Definition of Life: Autonomy and Open-Ended Evolution. Origins Life Evol. Biospheres 2004, 34, 323-346. [CrossRef]

567. Benner, S.A. Defining Life. Astrobiology 2010, 10, 1021-1030. [CrossRef] [PubMed]

568. Bich, L.; Green, S. Is defining life pointless? Operational definitions at the frontiers of biology. Synthese 2018, 195, 3919-3946. [CrossRef]

569. Amilburu, A.; Moreno, Á.; Ruiz-Mirazo, K. Definitions of life as epistemic tools that reflect and foster the advance of biological knowledge. Synthese 2021, 198, 10565-10585. [CrossRef]

570. Varela, F.; Maturana, H.R.; Uribe, R. Autopoiesis: The organization of living systems, its characterization and a model. BioSystems 1974, 5, 187-196. [CrossRef]

571. Luisi, P.L. Autopoiesis: A review and a reappraisal. Naturwissenschaften 2003, 90, 49-59. [CrossRef] [PubMed]

572. Luisi, P.L. The Minimal Autopoietic Unit. Origins Life Evol. Biospheres 2014, 44, 335-338. [CrossRef] [PubMed] 